\title{
Desordem e Competição em Modelos de Rede - Resultados Exatos
}

\author{
Masayuki O. Hase
}

INSTITUIODE FISICA

Serviço de Riblioteca e Fuformaçá

Tombo: T4376
Tese apresentada ao Instituto de Física da Universidade de São Paulo para a obtenção do título de Doutor em Ciências

Orientador: Prof. Dr. Silvio R. Salinas

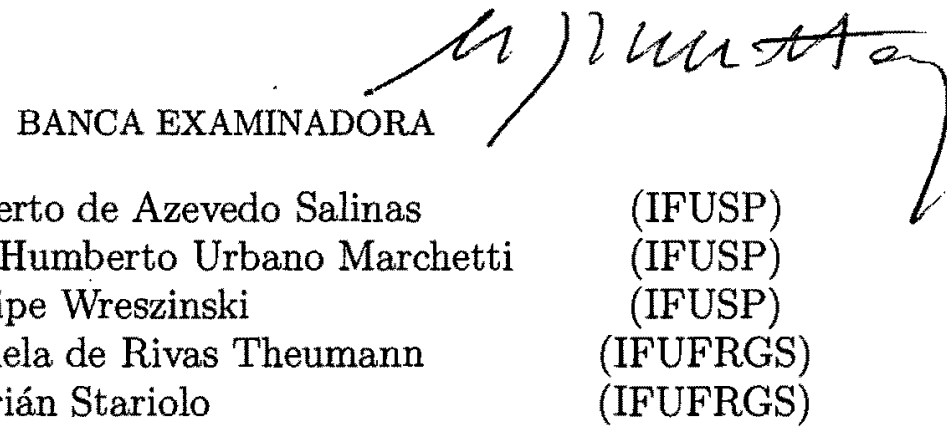

Prof. Dr. Silvio Roberto de Azevedo Salinas

Prof. Dr, Domingos Humberto Urbano Marchetti

Prof. Dr. Walter Felipe Wreszinski

Prof. Dr. Alba Graciela de Rivas Theumann

(IFUSP)

Prof. Dr. Daniel Adrián Stariolo

São Paulo

2005 


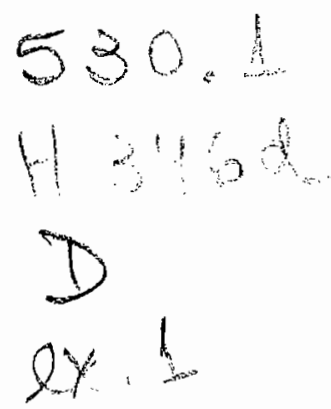

FICHA CATALOGRÁFICA

Preparada pelo Serviço de Biblioteca e Informação do Instituto de Física da Universidade de São Paulo

Hase, Masayuki 0

Desordem e Competição em Modelos de Rede Resultados Exatos.

São Paulo 2005.

Doutoramento - Universidade de São Paulo Instituto de Física - Depto. de Física Geral

Orientador: Prof. Dr. Silvio Roberto de Azevedo Salinas Área de Concentração: Física

Unitermos:

2 1. Física Teórica;

3 2. Mecânica Estatística Clássica;

$4 \quad 3$. Mecânica Estatística - Modelos Solúveis.

USP/IF/SBI-065/2005 


\section{Abstract}

Exact results are established for two distinct works. The first of them is an analysis of Langevin dynamics for a mean spherical model with competiting interactions, searching for non-equilibrium phenomena as aging and the violation of the fluctuation-dissipation theorem. The second work is a contribution to the ground state's phase diagram of a diluted ferromagnet under a random external field obeying a bimodal distribution; an analytic study, although partial, indicates a stability of the replica-symmetric solution.

\section{Resumo}

Resultados exatos são estabelecidos em dois trabalhos distintos. O primeiro tema é uma análise da dinâmica de Langevin para um modelo esférico médio com interações competitivas, investigando fenômenos de não-equilíbrio como "aging" e violação do teorema flutuação-dissipação. O segundo trabalho é uma contribuição ao diagrama de fases do estado fundamental de um ferromagneto diluído e sujeito a um campo externo aleatório regido por uma distribuição bimodal; uma abordagem analítica, embora parcial, indica uma estabilidade da solução réplica-simétrica. 
$\vdots$

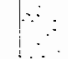




\section{Prefácio}

O leitor haverá de notar em breve que esta tese divide-se em duas partes, tanto no conteúdo quanto na apresentação. Dois temas, propostos em épocas e com propósitos distintos, que constituem uma fração da pesquisa desenvolvida durante o programa de doutoramento, foram selecionados para integrarem a síntese de cerca de três anos de trabalho. O primeiro assunto refere-se à dinâmica dissipativa de um modelo esférico médio; a motivação para o estudo deste problema tem origem no projeto inicial de pesquisa, entitulado "Efeitos de desordem, aperiodicidade e competição no modelo esférico", formulado no ano de 2002 e sob a orientação profícua do professor Silvio R. Salinas. O segundo tema da tese é uma contribuição ao diagrama de fases do estado fundamental para o modelo de Viana-Bray na presença de um campo aleatório bimodal, que se encontra no preprint cond-mat 0411682 e aceito para publicação na revista European Physical Journal B (2005); a motivação inicial e o desenvolvimento deste segundo trabalho foi possível em virtude da presença do professor Jairo R. L. de Almeida, que se manteve no Instituto de Física da Universidade de São Paulo - local onde o programa de doutoramento foi integralmente desenvolvido - na condição de professor visitante durante o ano de 2004. Esta é a razão para a coexistência na tese de assuntos aparentemente desconexos.

Quanto à apresentação, este trabalho é repartido em capítulos e apêndices, estes últimos correspondendo ao desenvolvimento explícito dos meandros técnicos apresentados nos primeiros. Com esta atitude, intenciona-se não quebrar o ritmo do leitor, em sua leitura dos capítulos, estorvando-o com páginas de cálculos que justificam os resultados mostrados. De fato, a presença desses passos técnicos ao longo do texto obriga-o, de certa forma, a acompanhar os pormenores que confeccionaram o produto final: sem dúvida, esta alternativa pode ser interpretada como um meio de valorizar os cálculos, que foram vitais para concluir o trabalho. Entretanto, julgou-se que isto desvalorizaria a tese por contribuir para a sua ininteligibilidade através das inúmeras interrupções da análise em andamento. A escolha de segregar todos os detalhes técnicos às seções entituladas "Apêndice" não deseja depreciar o conteúdo que ali foi direcionado; entende-se que os "Apêndices" constituam, tal como os capítulos, partes essenciais do trabalho, mas deixa-se ao leitor não somente a opção, mas também a responsabilidade quanto à decisão de lê-los (!).

O autor deseja manifestar seus sentimentos de gratidão ao professor Silvio R. Salinas pela importante orientação que enriqueceu muito esta tese ${ }^{1}$; ao professor Jairo R. L. de Almeida, responsável direto pela existência do capítulo 3 (e Apêndice B); ao professor Walter $F$. Wreszinski pelos comentários, que foram bastante úteis para o trabalho.

Os professores D. A. Stariolo e D. H. U. Marchetti fizeram contribuições importantes ao trabalho quando o autor estava envolvido na parte de revisão final desta tese.

Os colegas A. P. Vieira, A. Ghosh, D. J. Ribeiro, P. T. Muzy e T. A. S. Haddad, que trabalharam junto ao professor Salinas durante o desenvolvimento desta tese, devem ser registrados.

Considerações especiais devem ser feitas a D. K. N. de Almeida, I. Vantini e S. M. Ramos, e julgou-se pertinente mencionar, em reconhecimento, os nomes de A. J. Faria, A. T. N. Sardão, A. J. Maccori, E.

\footnotetext{
${ }^{1}$ Todos os erros, imprecisões e ímperfeições de inúmeras sortes, eventualmente presentes e oriundas de "alteraçōes secretas" realizadas sobre versões corrigidas pelo professor Salinas (que se submeteu à leitura paciente do texto por várias vezes), são, naturalmente, de total responsabilidade do autor.
} 
M. Santos, M. F. Cornelio, M. E. Y. K. Komori, N. O. Yokomizo, P. Vicente Filho, R. C. Alamino, R. Fresneda, S. R. Amaral, S. L. de Carvalho e T. P. Peixoto.

Agradecimentos à Fundação de Amparo à Pesquisa do Estado de São Paulo (FAPESP) pelo apoio financeiro (processo 02/00579-4).

$\mathrm{MOH}$

São Paulo

$\mathrm{X} / 2005$ 


\section{Sumário}

$\begin{array}{lc}\text { Prefácio } & \text { i }\end{array}$

$\begin{array}{lr}\text { Capítulo 1- Introdução } & 1\end{array}$

Capítulo 2- Dinâmica do modelo esférico médio com competição 3

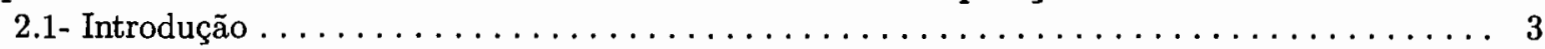

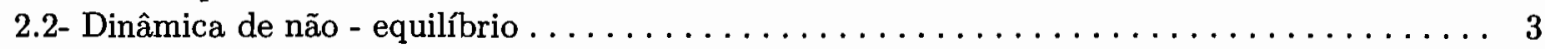

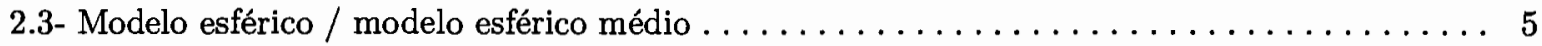

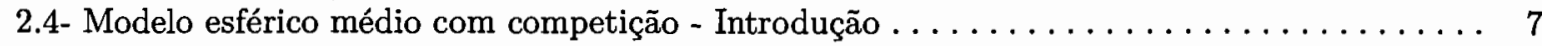

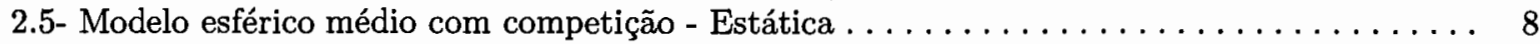

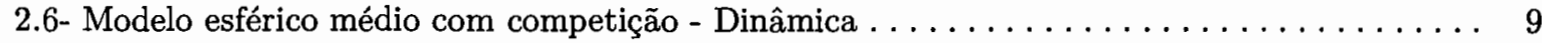

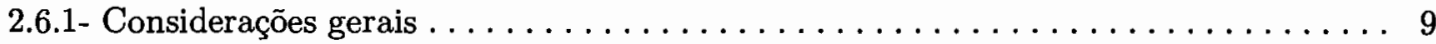

2.6.2- Dinâmica supercrítica ............................... 14

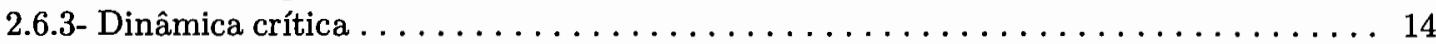

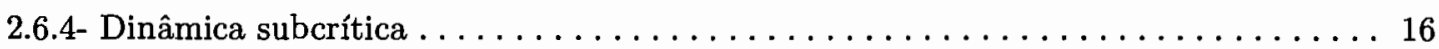

2.7- Considerações finais . . . . . . . . . . . . . . . . . . . . . 17

Capítulo 3- Modelo de Viana - Bray com campo aleatório 19

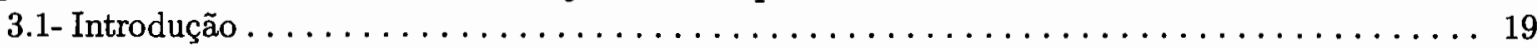

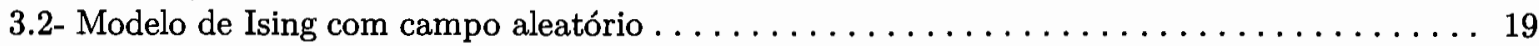

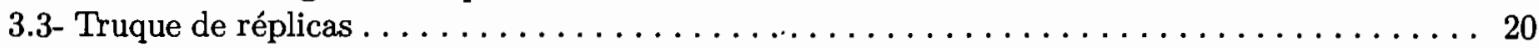

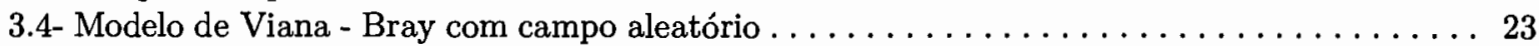

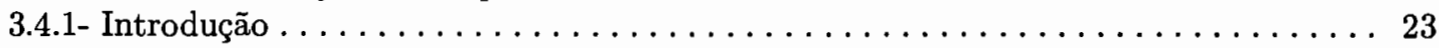

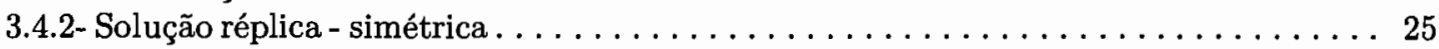

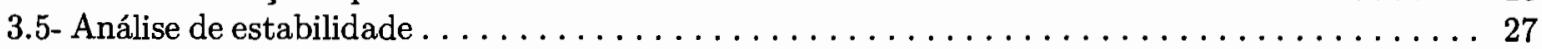

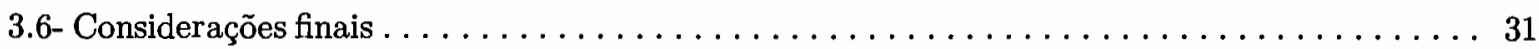

Apêndice A

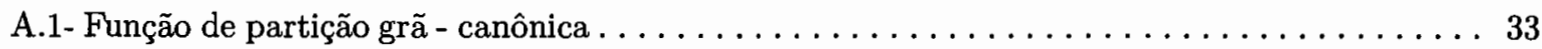

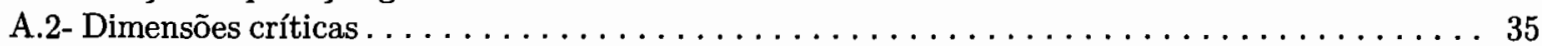

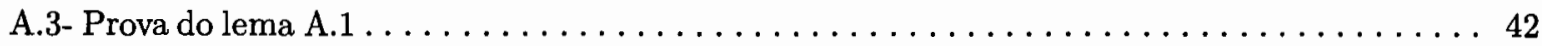

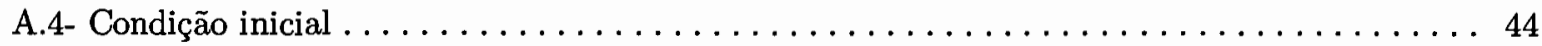

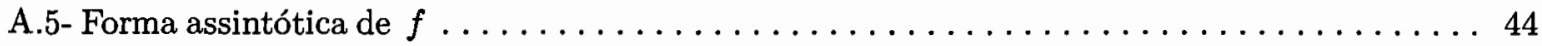

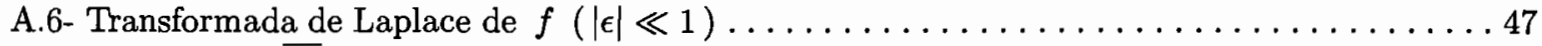

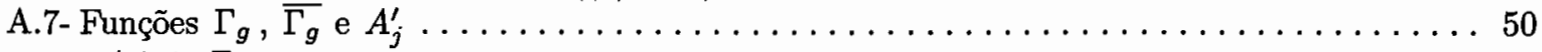

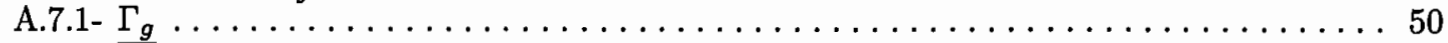

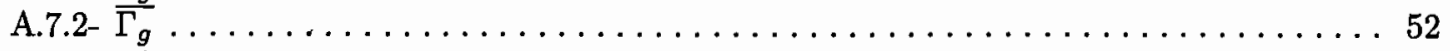

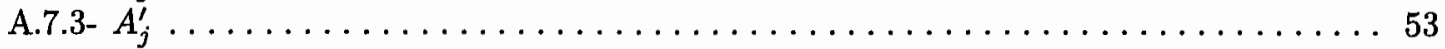

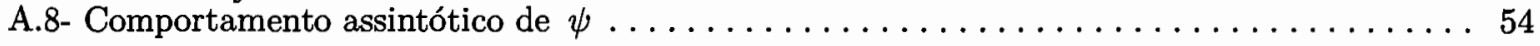




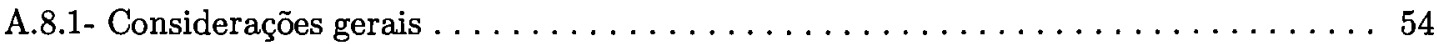

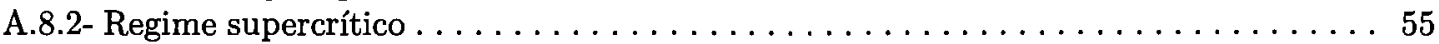

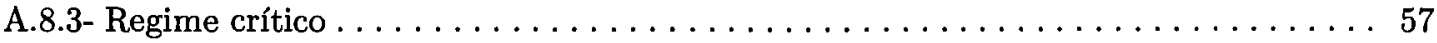

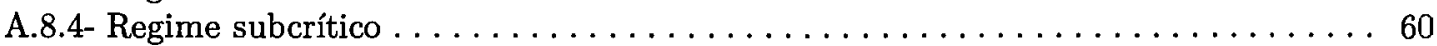

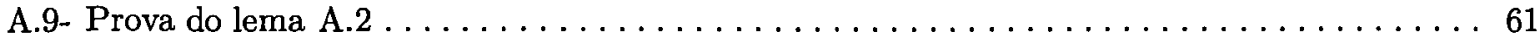

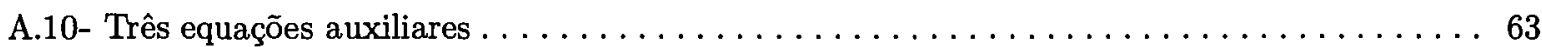

A.11- Autocorrelação, função resposta e coeficiente de flutuação - dissipação . . . . . . . . 64

A.11.1- Dinâmica supercrítica . . . . . . . . . . . . . . . . . . . . . 65

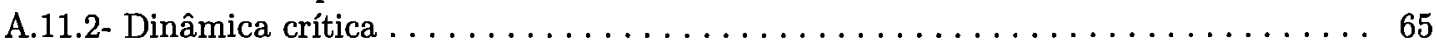

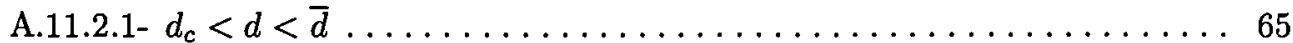

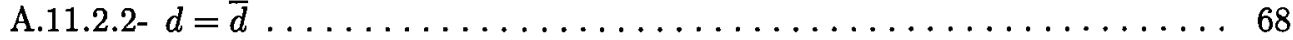

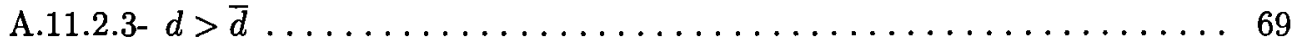

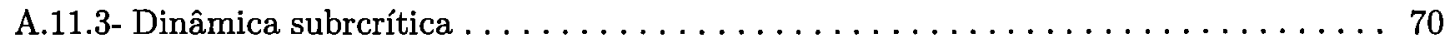

$\begin{array}{ll}\text { Apêndice B } & \mathbf{7 3}\end{array}$

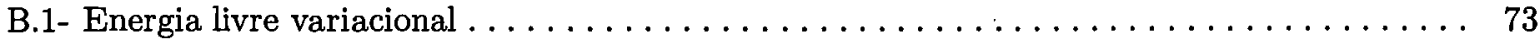

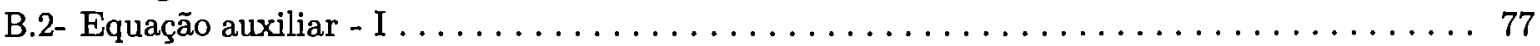

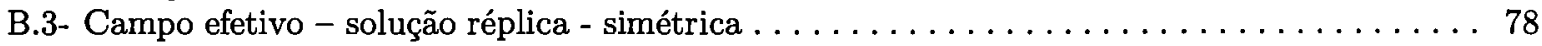

B.4- Energia livre estacionária - solução réplica - simétrica $\ldots \ldots \ldots \ldots \ldots \ldots \ldots \ldots \ldots . \ldots \ldots$

B.5- Relações auxiliares no estado fundamental $\ldots \ldots \ldots \ldots \ldots \ldots \ldots \ldots \ldots \ldots \ldots \ldots$

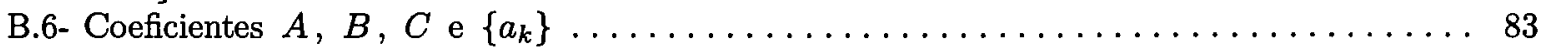

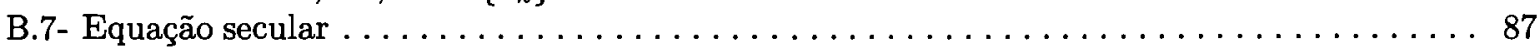

B.8- Prova do teorema de Mottishaw - de Dominicis . . . . . . . . . . . . . . . . . . 90

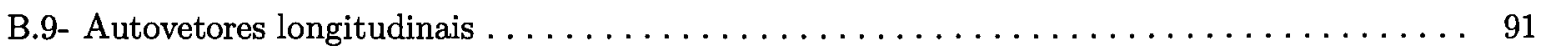

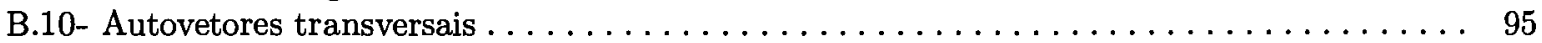

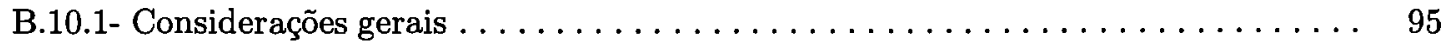

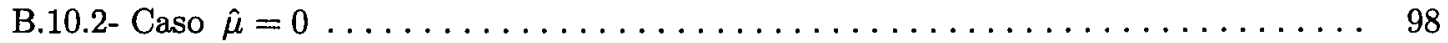

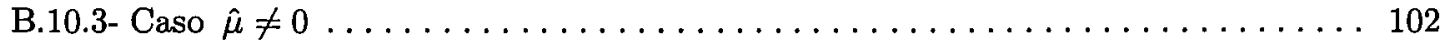

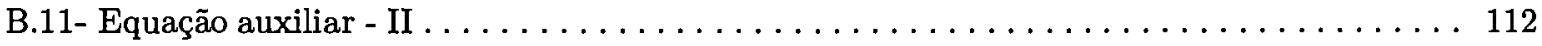

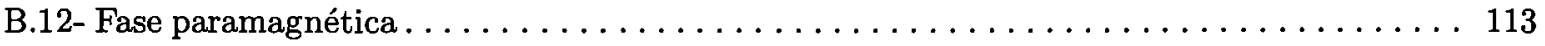

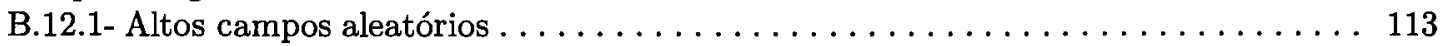

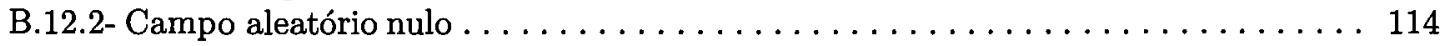

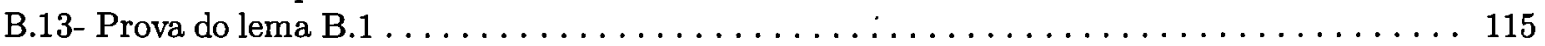

$\begin{array}{ll}\text { Referências Bibliográficas } & 117\end{array}$ 


\section{Capítulo 1}

\section{Introdução}

Duas escalas de tempo classificam, a grosso modo, o objeto de estudo da mecânica estatística aplicada à matéria condensada. A primeira, que é uma situação idealizada de tempos "infinitos" 2 , remete ao estudo do chamado "equilíbrio termodinâmico"; neste caso, do ponto de vista macroscópico, o sistema não mais evolui com o tempo - que deixa de desempenhar qualquer papel -, e as dinâmicas presentes restringemse a flutuações "pequenas"(termodinamicamente inacessíveis) em torno da situação macroscopicamente estática.

Na segunda escala de tempo, "finita", a evolução temporal do modelo em questão é o centro das atenções. O objețo de estudo aqui é o comportamento segundo o tempo (que agora é um parâmetro do problema) do sistema que ainda não atingiu um estado de equilíbrio. A mecânica estatística de não-equilíbrio tem sido desenvolvida através da proposta de modelos que inspirem novos conceitos ou estabelecendo relações entre fenômenos distintos, numa tentativa de melhor classificação. Seguindo esta linha de pesquisa, uma classificação de dinâmicas, provavelmente a mais aceita, foi proposta em 1977 por Hohenberg e Halperin[1], e dentre essas diversas classes, o chamado "modelo A" parece ser a mais estudada e popular em física. Trata-se de uma dinâmica dissipativa, sem conservação do parâmetro de ordem, e o representante desta classe é a equação de Langevin, que na linguagem moderna é expressa por

$$
\frac{\partial \phi}{\partial t}=-\frac{\delta H[\phi]}{\delta \phi}+\eta(t)
$$

onde $\eta$ é um ruído (aleatório), a função de Hamilton $H$ fornece os detalhes mecânicos do sistema e a evolução temporal de $\phi$ é examinada. A equação de Langevin é bastante conhecida e amplamente estudada desde a sua formulação para atacar o problema do movimento browniano e será também a geradora da dinâmica tratada nesta tese.

Deve-se esclarecer, inicialmente, que os problemas abordados aqui são tratados dentro do contexto da mecânica estatística clássica, no sentido de os spins serem $c$-funções do espaço (e também do tempo, no estudo da dinâmica) e não operadores sem a propriedade comutativa respeitada pela álgebra dos corpos reais. A estratégia para enfrentar esses problemas de mecânica estatítica consiste em, inicialmente, definir o modelo em um sistema de rede $\Lambda$ discreta e finita, de $|\Lambda|=N$ sítios, imersa em um conjunto maior (por exemplo, $\mathbb{Z}^{d}$ ). A descrição mecânica "microscópica" do modelo sob exame é feita em $\Lambda$ via uma função de Hamilton $H$, que depende da configuração $\left\{S_{x}\right\} \subset \Omega^{\Lambda}$ de spins ${ }^{3}$, com $\Omega \ni S_{x}$ sendo os valores possíveis que um spin pode assumir, a partir da qual se define a medida de Gibbs em volume finito

\footnotetext{
2Este "infinito", que evidenteménte é um abuso de linguagem, é inferior ao tempo necessário para qualquer forma de manifestação observável do teorema da recorrência de Poincaré (!).

${ }^{3}$ Por $S_{x}$, entende-se uma aplicação $S: \Lambda \rightarrow \Omega\left(x \mapsto S_{x}\right)$ que fornece o valor do spin na posição $x \in \Lambda$.
} 


$$
\mu_{\Lambda}(\beta):=\frac{e^{-\beta H\left(\left\{S_{x}\right\}\right)}}{Z_{\Lambda}(\beta)}
$$

onde a normalização $Z_{\Lambda}(\beta)$ indica que $\mu_{\Lambda}$ é uma medida de probabilidade. A convergência de $\mu_{\Lambda}$ a uma medida em volume infinito é a transição para o limite termodinâmico, e a equação de DobrushinLanford-Ruelle deve ser satisfeita para caracterizar uma situação de equilíbrio[2] dentro das fronteiras da mecânica estatística clássica.

Observa-se que tanto na dinâmica quanto na estática a conexão com um particular modelo de interesse é realizada pela mecânica, sintetizada nas funções de Hamilton presentes em (1.1) e (1.2). A escolha desta função decide, além do foco de atenção do trabalho, a complexidade da resolução do problema. Os casos supostamente mais simples, ditos "uniformes", referem-se, em geral, a modelos com estruturas homogêneas e isotrópicas.

Contudo, os materiais atraentes aos experimentais, ou muitos problemas de interesse teórico que constituem sistemas que supostamente os modelos de mecânica estatística pretende mimetizar, são dotados de impurezas e não-homogeneidades. O comportamento crítico destes materiais pode diferir de sua versão uniforme conforme a intensidade ou disposição de impurezas, que podem ser desordens, competições, e "imperfeições" de inúmeras sortes.

O advento do grupo de renormalização[3, 4] no início da década de 1970 proporcionou uma nova ferramenta para enfrentar os problemas referentes às possíveis mudanças na classe de universalidade originadas por impurezas introduzidas nos modelos. $O$ interesse por questões correlatas crescia, e publicações acerca desse assunto, mesmo aquelas que não continham a linguagem do grupo de renormalização, proliferaram. Exemplificando, o trabalho de Harris[5], que forneceu um critério heurístico para verificar se uma desordem seria suficientemente forte a ponto de remover o sistema de sua classe de universalidade "original" (caso uniforme), é bem conhecido; o modelo de Ising com campos aleatórios foi introduzido por Imry e $\mathrm{Ma}[6]$ em meados da década de 1970; no mesmo período, Edwards e Anderson propuseram um modelo de vidros de spin de curto alcance[7], e cuja versão de campo médio à la Curie-Weiss, um paradigma dos sistemas desordenados, foi apresentada por Sherrington e Kirkpatrick logo em seguida[8]; foram publicados, ainda, artigos que tratam de sistemas competitivos[9], que têm como características marcantes os ricos diagramas de fase com fases moduladas, cujos trabalhos teóricos precursores parecem remeter aos nomes de Yoshimori[10], Villain[11] e Kaplan[12], ainda no final da década de 1950.

A inserção de impurezas em modelos de mecânica estatística de equilíbrio e de não-equilíbrio compõe o conteúdo deste trabalho. A tese compõe-se de dois temas independentes. A primeira parte (capítulo 2) investiga os efeitos de competição sobre a dinâmica de um modelo esférico médio. Um caso particular do modelo tratado aqui já havia sido estudado[1.13], fornencendo a este trabalho um material de comparação que enriquece a análise.

O segundo assunto abordado na tese (capítulo 3), por outro lado, não tem como enfoque a evolução temporal de um sistema, mas preocupa-se com propriedades estáticas ou termodinâmicas, e o objeto de estudo é um ferromagneto que apresenta impurezas por diluição e presença de um campo aleatório. Mais precisamente, a desordem é temperada ${ }^{4}$, e a situação de equilíbrio é, a rigor, apenas putativa; porém, o problema é perfeitamente tratável pelos instrumentos da mecânica estatísitca de equilíbrio.

Os comentários de caráter mais específico são legados às introduções contidas nos capítulos. Os apêndices A e B expõem, respectivamente, os detalhes técnicos implícitos nos capítulos 2 e 3.

\footnotetext{
${ }^{4}$ Pelo limite de desordem temperada, entende-se que o tempo característico de acomodação da desordem é bem superior ao tempo característico de observação (em outros termos, a desordem permanece congelada durante uma experiência); comentários adicionais no capítulo 3.
} 


\section{Capítulo 2}

\section{Dinâmica do modelo esférico médio com competição}

\section{1- Introdução}

Sistemas em equilíbrio termodinâmico apresentam a propriedade de invariância por translação temporal. Em tais circunstâncias, funções de dois tempos $t^{\prime}$ (tempo de espera) e $t\left(\geq t^{\prime}\right)$, como a autocorrelação $C\left(t, t^{\prime}\right)$ ou a função resposta $R\left(t, t^{\prime}\right)$, dependem somente da diferença $\tau:=t-t^{\prime}$. Quando são submetidos a uma perturbação que os remove do estado estacionário, a invariância supracitada pode ser quebrada; como conseqüência, resultados pertencentes à mecânica estatística de equilíbrio como o teorema flutuação-dissipação podem não ser mais aplicáveis. O sistema pode, ainda, decair mais lentamente para algum estado quanto maior for o tempo de espera, caracterizando o fenômeno de "aging" (envelhecimento).

A fim de contemplar fenômenos dinâmicos em modelos competitivos, e sem desordem, recorreu-se a uma versão esférica do modelo ANNNI. (Axial Next-Nearest-Neighbor Ising). A escolha de modelos esféricos (médios) justifica-se pela possibilidade de obter resultados analíticos em situações "suficientemente simples" para qualquer dimensão; ademais, esta forma de resultados exatos para uma dinâmica competitiva parece não ter sido muito explorada pela literatura.

Optou-se por estudar uma dinâmica dissipativa, representada pela equação de Langevin. A situação experimental mimetizada aqui é um "quench" a partir de uma temperatura "efetivamente infinita" (altamente desordenada). As funções de autocorrelação e resposta são calculadas e analisadas em alguns regimes de tempo, investigando-se, ainda, a validade do teorema flutuação-dissipação.

Os tópicos mais importantes para o trabalho acerca da dinâmica de quasi-equilíbrio são expostos na seção 2.2, seguida de uma revisão do modelo esférico e esférico médio (seção 2.3). A seção 2.4 é dedicada a apresentar o modelo deste trabalho cujas partes estática e dinâmica são analisadas nas seções 2.5 e 2.6, respectivamente. As considerações finais são apresentadas na última seção, 2.7.

\section{2- Dinâmica de não-equilíbrio}

Dentre as diversas dinâmicas existentes na literatura, a de Langevin parece ser a mais popular. A equação de Langevin pertence a uma classe de dinâmicas com parâmetros de ordem dissipativos chamados "modelo A", na classificação de Hohenberg e Halperin[14]. Neste caso, a evolução temporal de alguma grandeza está sujeita a uma força dissipativa e um ruído externo aleatório, e a interação entre estas duas dá origem, numa situação de equilíbrio térmico, ao conhecido teorema flutuação-dissipação.

O propósito deste capítulo é investigar efeitos de não-equilíbrio na dinâmica de Langevin através de 
duas funções de dois tempos, a autocorrelação e a função resposta. Estas duas funções dependem de dois instantes de tempo da evolução do sistema, $t$ e $t^{\prime}$ (tempo de espera), com $t^{\prime} \leq t$, sendo que em situações de equilíbrio esta dependência dá-se somente via a diferença $\tau:=t-t^{\prime}$; esta propriedade caracteriza a invariância por translação temporal.

Os sistemas em equilíbrio termodinâmico são, pois, temporalmente homogêneos, mas o mesmo comentário não pode ser feito em circunstâncias fora do equilíbrio. Em particular, duas propriedades típicas desta situação vêm chamando a atenção nos últimos anos. A primeira é o "aging" (envelhecimento), um fenômeno em que a evolução temporal do sistema (ou algum observável) passa a depender de sua história. Ilustrando com os vidros de spin, o paradigma dos sistemas desordenados em matéria condensada, o tempo de relaxação (rumo ao equilíbrio) decai com uma lei de potência (e não exponencialmente como em sistemas ordenados), tornando clara a dependência do comportamento dinâmico com $t^{\prime}$, visto que o decaimento ao equilíbrio passa a ser um processo demorado. Esse tempo de relaxação pode superar, em várias ordens de grandeza, um tempo típico de observação.

Aparentemente, não há um princípio geral que explique as causas do "aging", mas se conhecem alguns fatores que favorecem a ocorrência deste fenômeno. Em sistemas desordenados, uma possível explicação é devido a Bouchaud[15], que associou a questão do envelhecimento aos tempos médios (à divergência destes) de permanência do sistema nas numerosas configurações metaestáveis, que correspondem a vales de energia livre onde o sistema pode ser aprisionado ("trap model" de Bouchaud).

Alternativamente à justificativa acima, a riqueza de armadilhas no perfil da energia livre não é a única condição para a realização do "aging": um perfil plano também pode ser responsável por esse fenômeno $[16,17]$. Observou-se também que a combinação de barreiras de energia livre altas com regiões planas extensas de energia, como nos modelos de campo médio[18, 19, 20,21,22], ou ainda razões de natureza entrópica[23] também são aptos a provocarem "aging". Nota-se, portanto, que o fenômeno dinâmico de "aging" não é observado somente em sistemas desordenados. Existem artigos na literatura que evidenciam a sua ocorrência em sistemas sem desordem e sem frustração através de tratamentos analíticos $[24,25,26,27]$.

O "aging" na autocorrelação e na função resposta tem implicações diretas em um resultado envolvendo estas duas grandezas; embora estas funções de dois tempos, definidas na seção 2.6.1, não sejam independentes na mecânica estatística de equilíbrio, sendo ligadas pelo teorema flutuação - dissipação,

$$
\frac{\partial C\left(t, t^{\prime}\right)}{\partial t^{\prime}}=T R\left(t, t^{\prime}\right)
$$

o elo pode sofrer alterações em situações de não-equilíbrio, levando à segunda propriedade típica para estes sistemas, que é a violação do teorema flutuação - dissipação, que juntamente com o fenômeno de "aging" tem recebido uma atenção especial. A equação

$$
\frac{\partial C\left(t, t^{\prime}\right)}{\partial t^{\prime}}=\frac{T}{X\left(t, t^{\prime}\right)} R\left(t, t^{\prime}\right)
$$

que recupera o teorema quando o coeficiente de flutuação - dissipação, $X\left(t, t^{\prime}\right)$, for igual a 1 , tem substituído (2.1) em problemas fora do equilíbrio. A forma da equação sugere a introdução de uma temperatura efetiva, $T / X\left(t, t^{\prime}\right)$, do sistema. Uma revisão acerca de "aging" e violação do teorema flutuação - dissipação pode ser encontrada em [28].

Uma hipótese importante[20], mas plausível, a ser feita sobre as funções de dois pontos é supor que elas decaiam por um longo tempo $t$ após $t^{\prime}$. A conseqüência disto é o sistema apresentar memória fraca de sua história para tempos curtos, embora os efeitos do decaimento sejam perceptíveis quando se integram as informações ao longo de um tempo grande. Esta propriedade direciona a análise para a dinâmica de tempos longos, além de permitir ignorar os detalhes para $t^{\prime}$ pequenos. Este trabalho, então, preocupará somente com a dinâmica assintótica de $t^{\prime}$ (e, por conseqüência, também de $t$ ) grande. 
Conforme mencionado anteriormente, a situação experimental a ser mimetizada aqui é um "quench" a partir de um estado altamente desordenado (temperatura efetivamente infinita) para uma temperatura $T$. Três tipos de dinâmica, supercrítica, crítica e subcrítica, podem ser consideradas, dependendo do valor de $T$ em relação à temperatura crítica $T_{c}$. Esspera-se que no caso supercrítico, pelo fato do sistema ainda permanecer em um estado de "altas temperaturas", o equilíbrio seja restaurado em um tempo "não muito longo" - no sentido de não se observar fenômenos de não-equilíbrio em um tempo assintoticamente grande (vide seção 2.6.2). As situações interessantes, que permitem contemplar os fenômenos dinâmicos de não-equilíbrio ("aging" e violação do teorema flutuação - dissipação), ocorrem nos casos crítico e subcrítico.

A dinâmica de Langevin, em modelos esféricos médios para sistemas sem desordem e frustração foi estudada por vários autores em diferentes contextos, sendo que em todos eles os fenômenos supracitados foram observados. Zippold et al. [24] consideraram um ferromagneto de curto alcance (e também um vidro de spin) como objeto de estudo, identificando os regimes de tempo para os quais se observa "aging" e a violação do teorema flutuação - dissipação; Godrèche e Luck [25] realizaram uma análise mais completa, analisando as dinâmicas supercrítica, crítica e subcrítica de um ferromagneto de curto alcance; em Cannas et al.[27] há uma análise para um ferromagneto com interações de longo alcance, com resultados para o comportamento da autocorelação também para uma dinâmica conservativa (o "modelo B", segundo Hohenberg e Halperin) no estado fundamental. Já Picone e Henkel [26] procuram considerar condições iniciais mais gerais em sua análise de um ferromagneto.

A dinâmica de Langevin de sistemas desordenados, mais especificamente do modelo de SherringtonKirkpatrick esférico, foi estudada por Cugliandolo e Dean na ausência [16] e presença [29] de campo externo.

Os artigos acima são amostras de estudo atual; aparentemente, não há um estudo analítico acerca de sistemas competitivos ${ }^{5}$. Tais sistemas podem ser representados por modelos competitivos do tipo ANNNI, e uma versão esférica generalizada deste modelo é objeto de análise neste trabalho.

\section{3- Modelo esférico / modelo esférico médio}

Apesar da solução de Onsager[31] do modelo de Ising em duas dimensões representar uma contribuição notável, a complicação técnica envolvida constitui um obstáculo na elucidação dos diversos aspectos dos fenômenos críticos. Neste ambiente, menos de uma década após a publicação do trabalho de Onsager, Kac apresentou uma corruptela do modelo de Ising, que foi resolvida por Berlin[32, 33]. Neste novo modelo, os spins deixam de assumir valores discretos para se tornarem variáveis contínuas, e a sua função de partição numa rede finita $\Lambda$, com $N$ sítios, imersa em uma rede $d$-dimensional $\mathbb{Z}^{d}$, é definida por

$$
Z_{\Lambda}(\beta)=\int_{\mathbb{R}^{\Lambda}} \exp \left[-\beta H\left(\left\{S_{x}\right\}\right)\right] \delta\left(\sum_{x \in \Lambda} S_{x}^{2}-N\right) \prod_{x \in \Lambda} d S_{x},
$$

onde $S_{x}$ assume valor na reta real para cada ponto $x$ da rede $\Lambda$ e $\beta$ é o inverso da temperatura $T$ do banho térmico; mais especificamente, $\beta:=\left(k_{B} T\right)^{-1}$, sendo $k_{B}$ a constante de Boltzmann.

A distribuição delta em (2.3), que introduz de forma microcanônica o termo $\sum_{x \in \Lambda} S_{x}^{2}$, garante a convergência da integral através do vínculo esférico

$$
\sum_{x \in \Lambda} S_{x}^{2}=N
$$

que confina os spins no intervalo $[-\sqrt{N}, \sqrt{N}] \subset \mathbb{R} .{ }^{6}$ Esta regra de soma é naturalmente satisfeita para spins de Ising.

\footnotetext{
${ }^{5} \mathrm{O}$ trabalho [30], que estuda um modelo competitivo, utiliza simulações numéricas.

${ }^{6} \mathrm{Em}$ outro modelo, o gaussiano[33], os spins assumem valores em toda a reta real, $e_{\text {, }}$ embora estejam sujeitos a um peso gaussiano, a função de partição não é definida para baixas temperaturas.
} 
Berlin e Kac analisaram o modelo esférico para um ferromagneto com interações de primeiros vizinhos em uma, duas e três dimensões, determinando a dimensão crítica igual a dois, abaixo da qual não se observa uma transição de uma fase ferromagnética para uma fase desordenada segundo a temperatura. Logo após a publicação de Berlin e Kac, Lewis e Wannier[34] propuseram uma variante do modelo esférico, em que $\sum_{x \in \Lambda} S_{x}^{2}$ entra canonicamente na função de partição. Nesta situação, a equação (2.3) é substituída pela função de partição grã-canônica

$$
\Xi_{\Lambda}(\beta, \mu)=\int_{\mathbb{R}^{\Lambda}} \exp \left[-\beta H\left(\left\{S_{x}\right\}\right)-\beta \mu \sum_{x \in \Lambda} S_{x}^{2}\right] \prod_{x \in \Lambda} d S_{x},
$$

onde $\mu$, o potencial químico, atua como um multiplicador de Lagrange, assegurando, apenas em média, o vínculo esférico,

$$
\left\langle\sum_{x \in \Lambda} S_{x}^{2}\right\rangle:=-\frac{1}{\beta} \frac{\partial}{\partial \mu} \ln \Xi_{\Lambda}(\beta, \mu)=N
$$

em contraste com (2.4). O símbolo $\langle\cdot\rangle$ indica média térmica.

Lewis e Wannier, embora inicialmente houvessem admitido a equivalência entre o modelo esférico e o modelo esférico médio[35], retrataram-se logo em seguida, percebendo que a equivalência seria apenas nas propriedades termodinâmicas dos modelos [35], e que grandezas como correlações não seriam necessariamente idênticas em ambos. Esta discrepância, relacionada à questão da equivalênica entre ensembles distintos (microcanônico e canônico), não é surpreendente.

Apesar de ter sido concebido e inspirado pelo modelo de Ising, o modelo esférico encontrou uma correspondência direta com o modelo $n$-vetorial. Esta equivalência, inicialmente sugerida por Stanley [36] no final da década de 1960, foi refutada por Helfand [37]. Este último mostrou (corretamente) que o argumento de Stanley não era válido em baixas temperaturas, e concluiu, estendendo (erroneamente) seu resultado, afirmando que a equivalência entre o modelo esférico e o modelo $n$-vetorial era falsa na região subcrítica de temperatura. Uma prova da correspondência foi apresentada logo em seguida por Kac e Thompson [38] e, anos mais tarde, por Shcherbina [39]. O primeiro artigo, porém, contém pontos obscuros em sua prova, apontados por Kunz e Zumbach [40]. A palavra final da equivalência entre os modelos esférico e $n$-vetorial parece, pois, ser dada pelo trabalho de Shcherbina. Uma revisão do assunto foi publicada por Khorunzhy e colaboradores [41].

$\mathrm{O}$ sucesso do modelo esférico reflete-se na sua solubilidade analítica em alguns casos importantes. $\mathrm{O}$ modelo com interações de curto alcance ${ }^{7}$ (de primeiros vizinhos, ferromagnético) foi mencionado acima. Para interações de longo alcance, com potenciais que decaem algebricamente com a distância, o trabalho de Joyce[42] estuda a influência da intensidade do decaimento na criticalidade do modelo; este mesmo autor tem outras contribuições para o modelo esférico, sendo uma delas um artigo de revisão acerca do assunto[43].

Já no contexto dos sistemas desordenados, a versão esférica do modelo de Sherrington-Kirkpatrick foi estudada por Kosterlitz et al.[44]. A integral de troca, obedecendo uma distribuição de probabilidades do tipo Gauss, permite invocar, dentro do contexto de matrizes aleatórias, a lei do semi-círculo para estabelecer a distribuição de seus autovalores e assim determinar a energia livre sem recorrer ao método das réplicas (este truque é apresentado no capítulo 3). Neste caso, o modelo esférico revelou-se um ótimo laboratório para testar o truque das réplicas, indicando a coincidência dos resultados obtidos por ambos os métodos.

O comportamento crítico do modelo esférico é, pois, analiticamente solúvel para sistemas "suficientemente simples" - embora importantes. Foram citados, aqui, alguns trabalhos referentes à termodinâmica

\footnotetext{
${ }^{7}$ O conceito de "curto alcance" pode ser controverso no modelo esférico, visto que o vínculo esférico estabelece uma relação entre todos os spins dos sítios; aqui, a classificação quanto ao alcance da interação é decidida somente pelas propriedades da integral de troca - se esta é uma função de suporte compacto, a interação é entendida como sendo de "curto alcance".
} 
dos modelos. Como foi mencionado na seção anterior, há publicações que trataram da análise dinâmica, e a sua solubilidade analítica foi novamente útil para enriquecer o estudo.

No presente trabalho, o modelo esférico médio é examinado, estimulado pela possibilidade de tratar analiticamente o comportamento crítico (estático e dinâmico). A implementação da competição é inspirada no modelo ANNNI, fato este evidenciado abaixo.

\section{4- Modelo esférico médio com competição - Introdução}

Na literatura, a lista de publicações que trata de investigar fenômenos de não-equilíbrio - como "aging" e violação do teorema flutuação-dissipação - em sistemas de spins contínuos é significativa, e o foco de atenção divide-se em duas áreas. A primeira, que enfoca sistemas desordenados, estuda o modelo de Sherrington-Kirkpatrick e a abordagem é via campo médio; é o caso de [16] e [24]. A segunda, representada por modelos ferromagnéticos com interações de curto[24, 25] (de primeiros vizinhos) e longo[27] (interações que decaem algebricamente com a distância) alcance, trata de examinar sistemas sem desordem e sem frustração. Uma abordagem analítica na dinâmica de um modelo competitivo, entretanto, parece estar ausente na literatura; é neste ponto que esta tese pretende contribuir.

Com relação às propriedades estáticas, a primeira introdução e estudo de efeitos competitivos no modelo esférico parece remeter ao trabalho de Kalok e Obermair[45], que propõe confrontar, isotropicamente, interações ferromagnéticas de primeiros vizinhos com uma interação antiferromagnética de segundos vizinhos. Neste trabalho, uma atenção especial é dada à existência de duas fases ordenadas (ferromagnética e helicoidal) com cálculos incipientes caracterizando suas interfaces. Posteriormente, o encontro entre essas fases e a fase desordenada foi nomeado "ponto de Lifshitz" por Hornreich et al. [46]. Estes autores realizaram também um levantamento de um diagrama de fases para uma versão esférica do modelo ANNNI[47]. Uma revisão do assunto pode ser encontrada em [48].

Seja $\Lambda_{N}:=\{-L, \cdots, L+1\}^{d} \subset \mathbb{Z}^{d}$ uma rede hipercúbica finita em $d$ dimensões, com parâmetro de rede igual a 1 e englobando $N=(2 L+1)^{d}$ pontos. As condiçōes de contorno são periódicas, caracterizando $\Lambda_{N}$ com um toro em $d$ dimensões. Cada ponto $x$ da rede será descrito pelo sistema cartesiano de coordenadas, $x=\left(x_{1}, \cdots, x_{d}\right)$. Denota-se por $e_{i}$,

$$
e_{i}:=(0, \cdots, 0,1,0, \cdots, 0),
$$

com o 1 ocupando a $i$-ésima coordenada, os versores cartesianos em $\Lambda_{N}$. Das $d$ direções linearmente independentes e ortogonais entre si (imersas na estrutura do sistema cartesiano), selecionam-se arbitrariamente $m$ ao longo das quais as interações competitivas podem ser introduzidas; estas $m$ direções, sem perda de generalidade, podem ser representadas pelo conjunto de versores $\left\{e_{1}, \cdots, e_{m}\right\}$. Nas $d-m$ direções restantes, descritas pelo conjunto $\left\{e_{m+1}, \cdots, e_{d}\right\}$, não serão introduzidas competições.

A função de Hamilton é escrita como

$$
H_{N}\left(\left\{S_{x}\right\}\right)=-\frac{1}{2} \sum_{x, x^{\prime} \in \Lambda_{N}} J\left(x, x^{\prime}\right) S_{x} S_{x^{\prime}}
$$

onde $S_{x} \in \mathbb{R}$ e a interação de troca $J\left(x, x^{\prime}\right)$ é dada por

$$
J\left(x, x^{\prime}\right)=\left\{\begin{array}{cll}
R J & , x-x^{\prime}= \pm e_{i} & i \in\{1, \cdots, m\} \\
S J \quad, x-x^{\prime}= \pm 2 e_{i} & i \in\{1, \cdots, m\} \\
J \quad, x-x^{\prime}= \pm e_{i} & i \in\{m+1, \cdots, d\} \\
0 \quad, \quad x-x^{\prime} \neq \pm e_{i}, \pm 2 e_{j} & i \in\{1, \cdots, d\} \quad \text { e } j \in\{m+1, \cdots, d\}
\end{array}\right.
$$


que é uma generalização natural do análogo esférico do modelo ANNNI. Neste trabalho tomou-se $J>0$. Apenas ao longo das direções de $e_{1}$ a $e_{m}$ têm-se interações de segundos vizinhos.

A situação de competição é realizada quando $R>0$ e $S<0$, ou $R<0$ e $S<0$. No primeiro caso, a frustração decorre do conflito entre inter ações ferromagnéticas de primeiros vizinhos e antiferromagnéticas de segundos vizinhos; por outro lado, no segundo caso, ambas as interações, de primeiros e segundos vizinhos, são antiferromagnéticas.

A razão por preferir o modelo esférico médio $((2.5)$ e (2.6)) ao modelo esférico tradicional $((2.3)$ e (2.4)) nesta tese será esclarecida mais adiante, na análise da dinâmica.

\section{5- Modelo esférico médio com competição - Estática}

Esta seção é dedicada a apresentar os resultados termodinâmicos essenciais à análise dinâmica. Muitos dos aspectos estáticos do modelo já foram explorados na literatura $[45,46,47,48]$, e o que se pretende fazer aqui é reunir, de forma condensada, somente as informações que interessam.

A termodinâmica do sistema é proveniente da função de partição grãa-canônica (2.5). Considerando a condição de contorno periódica, é conveniente realizar a análise estática no espaço recíproco, onde se tem (Apêndice A.1)

$$
\Xi_{\hat{\Lambda}_{N}}(\beta, \mu)=\left(\frac{\pi}{\beta}\right)^{\frac{N}{2}} \prod_{k \in \hat{\Lambda}_{N}}\left[\mu-\frac{1}{2} \hat{J}(k)\right]^{-\frac{1}{2}},
$$

onde $\hat{J}(k)$ é a transformada de Fourier de $J\left(x, x^{\prime}\right)$,

$$
\hat{J}(k)=2 J\left[R \sum_{i=1}^{m} \cos \left(k_{i}\right)+S \sum_{i=1}^{m} \cos \left(2 k_{i}\right)+\sum_{i=m+1}^{d} \cos \left(k_{i}\right)\right], \quad k \in \hat{\Lambda}_{N}
$$

O vetor $k$ é um elemento do conjunto $\hat{\Lambda}_{N}:=\left\{\frac{\pi x}{L+1}, x \in \Lambda_{N}\right\}$, que é a primeira zona de Brillouin. A condição necessária para a existência da equação (2.10) restringe o valor de $\mu$, que deve satisfazer, no limite termodinâmico,

$$
\mu \geq \mu_{c}:=\sup _{k \in \hat{[}-\pi, \pi]^{d}}\left\{\frac{1}{2} \hat{J}(k)\right\},
$$

definindo o potencial químico crítico $\mu_{c}$, assim como o vetor de onda $k_{c}$ (que satisfaz $\mu_{c}=\frac{1}{2} \hat{J}\left(k_{c}\right)$ ), dado por

$$
k_{c}=(\underbrace{q_{c}, \cdots, q_{c}}_{m}, \underbrace{0, \cdots, 0}_{d-m})
$$

onde

$$
q_{c}=\left\{\begin{array}{lllll}
0 & , & R>0 & \text { e } & S>-\frac{R}{4} \\
\pi & , & R<0 & \text { e } & S>-\frac{R}{4} \\
\pm \phi & , & S<-\frac{|R|}{4}
\end{array}\right.
$$


onde $\phi:=\arccos \left(-\frac{R}{4 S}\right)$.

A regra de soma (2.6) pode ser escrita, então, como

$$
\beta(\mu)=\int_{[-\pi, \pi]^{d}} \frac{d^{d} k}{2(2 \pi)^{d}} \frac{1}{\mu-\frac{1}{2} \hat{J}(k)} .
$$

A equação acima expressa a temperatura em função do potencial químico. Algumas propriedades de $\beta(\mu)$ podem ser facilmente verificadas:

(i) $\beta:\left[\mu_{c}, \infty\right) \rightarrow[0, \infty)$.

(ii) $\beta$ é monotonicamente decrescente com $\mu$.

(iii) $\beta$ é convexa.

A equação (2.15) é usada para determinar as dimensões críticas do sistema, que dependem de $m$, $d, R$ e $S .^{8}$ O estudo é realizado investigando a finitude da temperatura crítica $\beta_{c}:=\beta\left(\mu_{c}\right)$. Cabe observar que a imagem de $\beta$ percorre toda a reta real não-negativa na ausência de transição de fase $\left(\beta_{c}=\infty\right)$.

O cálculo das dimensões críticas $d_{c}$ e dimensões críticas superiores $\bar{d}$ (acima das quais os expoentes críticos passam a ser clássicos) é feito no Apêndice A.2, com os resultados organizados na tabela a seguir.

\begin{tabular}{|l|l|l|l|l|}
\hline \multirow{2}{*}{$m \neq 0, d-m \neq 0$} & $|R|+4 S \neq 0$ & Caso 1 & $d_{c}=2$ & $\bar{d}=4$ \\
\cline { 2 - 5 } & $|R|+4 S=0$ & Caso 2 & $d_{c}=\frac{4}{2-r}$ & $\bar{d}=\frac{8}{2-r}$ \\
\hline \multirow{2}{*}{$m \neq 0, d-m=0$} & $|R|+4 S \neq 0$ & Caso 3 & $d_{c}=2$ & $\bar{d}=4$ \\
\cline { 2 - 5 } & $|R|+4 S=0$ & Caso 4 & $d_{c}=4$ & $\bar{d}=8$ \\
\hline$m=0, d-m \neq 0$ & & Caso 5 & $d_{c}=2$ & $\bar{d}=4$ \\
\hline
\end{tabular}

Tabela 2.1: Dimensões críticas.

A tabela acima, com $r:=m / d$, contém as informações que permitem concluir a análise dinâmica, apresentada na seção a seguir.

\section{6- Modelo esférico médio com competição - Dinâmica}

\subsection{1- Considerações gerais}

Em modelos de spins, como em (2.8) com (2.9), o parâmetro "tempo" é ausente. O interesse desses modelos resume-se na sua análise estática, como apresentado na seção anterior. A dinâmica, portanto, deve ser imposta, sendo a sua escolha arbitrária. Conforme mencionado, uma classificação das dinâmicas possíveis foi proposta por Hohenberg e Halperin[14]; dentre as possibilidades, a dinâmica dissipativa (ou modelo A), sendo a mais popular, foi eleita para análise.

O representante típico desta classe de dinâmica é a equação de Langevin

$$
\frac{\partial S_{x}(t)}{\partial t}=-\frac{\delta}{\delta S_{x}(t)}\left\{H\left[S_{x}\right](t)+\mu \sum_{x \in \Lambda_{N}} S_{x}^{2}(t)\right\}+\xi_{x}(t),
$$

\footnotetext{
${ }^{8}$ Mais precisamente, de $m, d$ e da razão $S / R$.
} 
onde se introduz um conjunto de variáveis aleatórias $\left\{\xi_{x}(t)\right\}$ tal que

$$
\left\langle\xi_{x}(t)\right\rangle=0 \quad \text { e } \quad\left\langle\xi_{x}(t) \xi_{x^{\prime}}\left(t^{\prime}\right)\right\rangle=2 T \delta_{x, x^{\prime}} \delta\left(t-t^{\prime}\right),
$$

onde $\langle\cdot\rangle$ indica média térmica. Os fatores irrelevantes em (2.16) e (2.17) foram tomados como sendo 1. Notar que a participação do termo $\sum_{x \in \Lambda} S_{x}^{2}$ de forma microcanônica, como ocorre no modelo esférico, impede expressar a equação de Langevin da forma apropriada acima - (2.16). Por esta razão, o modelo esférico médio é preferido ao modelo esférico no estudo desta dinâmica.

Novamente, $\mu$ tem o papel de multiplicador de Lagrange, e aqui assegura o vínculo esférico para cada instante $t$.

Como no caso estático, a análise revelar-se-á mais adequada no espaço recíproco, onde a equação (2.16) é escrita como

$$
\frac{\partial \hat{S}_{k}(t)}{\partial t}=[\hat{J}(k)-2 \mu(t)] \hat{S}_{k}(t)+\hat{\xi}_{k}(t)
$$

com as equações em (2.17) transformadas em $\left\langle\hat{\xi}_{k}(t)\right\rangle=0$ e $\left\langle\hat{\xi}_{k}(t) \hat{\xi}_{-k}\left(t^{\prime}\right)\right\rangle=2 T \delta\left(t-t^{\prime}\right)$.

A solução formal de (2.18),

$$
\hat{S}_{k}(t)=\hat{S}_{k}(0) \exp \left[\hat{J}(k) t-\int_{0}^{t} 2 \mu\left(t^{\prime}\right) d t^{\prime}\right]+\int_{0}^{t} \exp \left[\hat{J}(k)\left(t-t^{\prime}\right)-\int_{t^{\prime}}^{t} 2 \mu\left(t^{\prime \prime}\right) d t^{\prime \prime}\right] \hat{\xi}_{k}\left(t^{\prime}\right) d t^{\prime}
$$

é o ponto de partida para definir grandezas como a autocorrelação e a função resposta. A solução da equação diferencial admite, naturalmente, a dependência com a condição inicial $\hat{S}_{k}(0)$.

Defina, agora, a função de autocorrelação $C\left(t, t^{\prime}\right)$,

$$
C\left(t, t^{\prime}\right):=\frac{1}{N} \sum_{x \in \Lambda_{N}}\left\langle S_{x}(t) S_{x}\left(t^{\prime}\right)\right\rangle=\frac{1}{N} \sum_{k \in \hat{\Lambda}_{N}} C_{k}\left(t, t^{\prime}\right),
$$

onde $C_{k}\left(t, t^{\prime}\right):=\left\langle\hat{S}_{k}(t) \hat{S}_{-k}\left(t^{\prime}\right)\right\rangle$, e a função resposta

$$
R\left(t, t^{\prime}\right):=\left.\frac{1}{N} \sum_{x \in \Lambda_{N}} \frac{\delta\left\langle S_{x}(t)\right\rangle}{\delta h_{x}\left(t^{\prime}\right)}\right|_{h=0}=\frac{1}{N} \sum_{k \in \hat{\Lambda}_{N}} R_{k}\left(t, t^{\prime}\right)
$$

onde $R_{k}\left(t, t^{\prime}\right):=\frac{\delta\left\langle\hat{S}_{k}(t)\right\rangle}{\delta h_{k}\left(t^{\prime}\right)}$ e $h$ é um campo auxiliar cuja finalidade é introduzir uma perturbação (pequena) que permita obter a resposta do sistema. As representações das funções de dois tempos no espaço de Fourier são dadas em seus respectivos terceiros membros nas equações (2.20) e (2.21). O coeficiente de flutuação - dissipação é definido por

$$
X\left(t, t^{\prime}\right):=\frac{T R\left(t, t^{\prime}\right)}{\partial_{t^{\prime}} C\left(t, t^{\prime}\right)} .
$$

O teorema flutuação - dissipação realiza-se quando - e somente quando - $X\left(t, t^{\prime}\right)=1$. A meta deste trabalho é detalhar os aspectos dinâmicos dessas duas funçōes de dois tempos; o estudo permite, ainda, verificar a validade do teorema flutuação - dissipação em situações fora do equilíbrio. O comportamento 
de $C\left(t, t^{\prime}\right)$ e $R\left(t, t^{\prime}\right)$, embora seja inteiramente determinado pela equação de movimento para os spins, (2.19), necessita de alguns meandros técnicos intermediários para uma solução analítica satisfatória.

Introduzindo o funcional

$$
\psi[\mu](t):=\exp \left[4 \int_{0}^{t} \mu\left(t^{\prime}\right) d t^{\prime}\right]
$$

que será denotado doravante por $\psi(t)$, chega-se a

$$
C_{k}\left(t, t^{\prime}\right)=\frac{1}{\sqrt{\psi(t) \psi\left(t^{\prime}\right)}}\left\{C_{k}(0,0) \exp \left[\hat{J}(k)\left(t+t^{\prime}\right)\right]+2 T \int_{0}^{t^{\prime}} \exp \left[\hat{J}(k)\left(t+t^{\prime}-2 t^{\prime \prime}\right)\right] \psi\left(t^{\prime \prime}\right) d t^{\prime \prime}\right\}
$$

com a informação acerca das condições iniciais contida em $C_{k}(0,0)$. A situação de interesse é, a partir de uma temperatura efetivamente infinita, resfriar o sistema, que se encontra, pois, em um estado inicial totalmente desordenado. Este caso implica $C_{k}(0,0)=1$ (Apêndice A.4).

O cálculo de $C\left(t, t^{\prime}\right)$ está sujeito à determinação do multiplicador de Lagrange (agora representado por $\psi[\mu](t)$ ), que pode ser determinado através do vínculo esférico $C(t, t)=1$ (vide $(2.6)$ e $(2.20)$ ), chegando-se a

$$
\psi(t)=\frac{1}{N} \sum_{k \in \hat{\Lambda}_{N}}\left\{e^{2 \hat{J}(k) t}+2 T \int_{0}^{t} e^{2 \hat{J}(k)\left(t-t^{\prime}\right)} \psi\left(t^{\prime}\right) d t^{\prime}\right\}
$$

onde a condição inicial $C_{k}(0,0)=1$ foi imposta. No limite termodinâmico $N \rightarrow \infty$, através de uma soma de Riemann, a equação (2.25) assume a forma de uma equação integral de Volterra,

$$
\psi(t)=f(t)+2 T \int_{0}^{t} f\left(t-t^{\prime}\right) \psi\left(t^{\prime}\right) d t^{\prime}
$$

onde

$$
f(t):=\int_{[-\pi, \pi]^{d}} \frac{d^{d} k}{(2 \pi)^{d}} e^{2 \hat{J}(k) t}=\left[I_{0}(4 J t)\right]^{d-m}\left[\frac{1}{\pi} \int_{0}^{\pi} e^{4 J t g(k)} d k\right]^{m},
$$

$I_{0}(x)$ é a função modificada de Bessel de ordem zero no ponto $x$ e $g(k)$,

$$
g(k):=R \cos k+S \cos (2 k),
$$

é a parte da integral de troca responsável pela interação de primeiros e segundos vizinhos.

O produto de convolução na integral em (2.26) sugere a resolução desta equação via transformadas de Laplace, que fornece

$$
\psi(t)=\frac{1}{2 \pi i} \int_{\sigma-i \infty}^{\sigma+i \infty} d s e^{s t} \frac{\mathcal{L}[f](s)}{1-2 T \mathcal{L}[f](s)}
$$


onde $\sigma$ é maior que a parte real de todos os pólos do integrando. A importância de $\psi(t)$ é evidenciada pelo fato de a autocorrelação,

$$
C\left(t, t^{\prime}\right)=\frac{1}{\sqrt{\psi(t) \psi\left(t^{\prime}\right)}}\left[f\left(\frac{t+t^{\prime}}{2}\right)+2 T \int_{0}^{t^{\prime}} d y f\left(\frac{t+t^{\prime}}{2}-y\right) \psi(y)\right]
$$

e a função resposta,

$$
R\left(t, t^{\prime}\right)=f\left(\frac{t-t^{\prime}}{2}\right) \sqrt{\frac{\psi\left(t^{\prime}\right)}{\psi(t)}},
$$

poderem ser por ela expressas. O comportamento dinâmico dessas funções de dois tempos, antes relacionadas à equação de movimento (2.19), está condicionado, agora, a $f(t)$ e $\psi(t)$. O interesse é analisar dois regimes de tempo, $1 \sim \tau \ll t^{\prime}$ e $1 \ll \tau \sim t^{\prime}$, como será visto mais adiante. Estes dois casos demandam determinar o comportamento assintótico (de grandes tempos) de $f$ e $\psi$. Para $f$ (Apêndice A.5),

$$
f(t) \sim K_{p} \frac{e^{2 \hat{J}\left(k_{c}\right) t}}{t^{\gamma_{p}}}
$$

onde $p$ indica o conjunto $p=\{R, S, m, d\}$, e portanto $K_{p}$ expressa a dependência do coeficiente $K$ com os parâmetros $R, S, m$ e $d$. O mesmo comentário vale para $\gamma_{p}=\gamma(R, S, m, d)$. Os valores para $K_{p}$ e $\gamma_{p}$ são listados abaixo, divididos segundo a classificação da tabela 2.1.

\begin{tabular}{|c|c|c|}
\hline & $K_{p}$ & $\gamma_{p}$ \\
\hline Caso 1 & $2^{m}(8 \pi J)^{-\frac{d}{2}}\left|g^{(2)}\left(q_{c}\right)\right|^{-\frac{m}{2}}$ & $\frac{d}{2}$ \\
\hline Caso 2 & $(8 \pi J)^{-\frac{2 d-m}{4}}\left(\frac{48}{\pi^{3}}\right)^{\frac{m}{4}} \Gamma\left(\frac{5}{4}\right)^{m}\left|g^{(4)}\left(q_{c}\right)\right|^{-\frac{m}{4}}$ & $\frac{2 d-m}{4}$ \\
\hline Caso 3 & $2^{d}(8 \pi J)^{-\frac{d}{2}}\left|g^{(2)}\left(q_{c}\right)\right|^{-\frac{d}{2}}$ & $\frac{d}{2}$ \\
\hline Caso 4 & $(8 \pi J)^{-\frac{d}{4}}\left(\frac{48}{\pi^{3}}\right)^{\frac{d}{4}} \Gamma\left(\frac{5}{4}\right)^{d}\left|g^{(4)}\left(q_{c}\right)\right|^{-\frac{d}{4}}$ & $\frac{d}{4}$ \\
\hline Caso 5 & $(8 \pi J)^{-\frac{d}{2}}$ & $\frac{d}{2}$ \\
\hline
\end{tabular}

Tabela 2.2: Valores para $K_{p}$ e $\gamma_{p}$.

A expansão assintótica de $\psi(t)$ (para $t$ grande) requer, antes, o comportamento de $\mathcal{L}[f](s)$ para $s$ pequeno (Apêndice A.6, equação (A.81), para o caso 5). Definindo $\epsilon:=s-2 \hat{J}\left(k_{c}\right)$, tem-se

$$
\mathcal{L}[f](s) \sim\left\{\begin{array}{ll}
\frac{F_{p} g_{p}}{\epsilon^{-\alpha_{p}}} & 0<d<d_{c} \\
F_{p}(-\ln \epsilon) & d=d_{c} \\
A_{1}-F_{p}\left|g_{p}\right| \epsilon^{\alpha_{p}} & d_{c}<d<\bar{d} \\
A_{1}-F_{p}(-\epsilon \ln \epsilon) & d=\bar{d} \\
A_{1}-F_{p} \epsilon & d>\bar{d}
\end{array} .\right.
$$


Novamente, $o$ índice $p$ nos coeficientes $F_{p}$ e $g_{p}$, e no expoente $\alpha_{p}$ indica a dependência destes em relação a $p=\{R, S, m, d\}$. Os valores que estes três assumem foram determinados pelo método descrito no Apêndice A.6, chegando-se aos resultados organizados na tabela 2.3, lembrando que $r:=m / d$.

\begin{tabular}{|c|c|c|c|}
\hline Casos & $F_{p}$ & $d$ & $\alpha_{p}, g_{p}$ \\
\hline \multirow[t]{2}{*}{ Caso 1} & $x$ & $d<2$ & \multirow{3}{*}{$\alpha_{p}=\frac{d-2}{2}$} \\
\hline & $2^{m}(8 \pi J)^{-1}\left|g^{(2)}\left(q_{c}\right)\right|^{-\frac{m}{2}}$ & $d=2$ & \\
\hline \multirow{3}{*}{$\begin{array}{c}m \neq 0 \\
d-m \neq 0 \\
|R|+4 S \neq 0\end{array}$} & $2^{m}(8 \pi J)^{-\frac{d}{2}}\left|g^{(2)}\left(q_{c}\right)\right|^{-\frac{m}{2}}$ & $2<d<4$ & \\
\hline & $2^{m}(8 \pi J)^{-2}\left|g^{(2)}\left(q_{c}\right)\right|^{-\frac{m}{2}}$ & $d=4$ & \multirow[t]{2}{*}{$g_{p}=\Gamma\left(\frac{2-d}{2}\right)$} \\
\hline & $A_{2}$ & $d>4$ & \\
\hline \multirow[t]{2}{*}{ Caso 2} & $(8 \pi J)^{-\frac{2 d-m}{4}}\left(\frac{48}{\pi^{3}}\right)^{\frac{m}{4}} \Gamma\left(\frac{5}{4}\right)^{m}\left|g^{(4)}\left(q_{c}\right)\right|^{-\frac{m}{4}}$ & $d<\frac{4}{2-r}$ & \multirow{5}{*}{$\alpha_{p}=\frac{2 d-m-4}{4}$} \\
\hline & $(8 \pi J)^{-1}\left(\frac{48}{\pi^{3}}\right)^{\frac{m}{4}} \Gamma\left(\frac{5}{4}\right)^{m}\left|g^{(4)}\left(q_{c}\right)\right|^{-\frac{m}{4}}$ & $d=\frac{4}{2-r}$ & \\
\hline \multirow{3}{*}{$\begin{array}{c}m \neq 0 \\
d-m \neq 0 \\
|R|+4 S=0\end{array}$} & $(8 \pi J)^{-\frac{2 d-m}{4}}\left(\frac{48}{\pi^{3}}\right)^{\frac{m}{4}} \Gamma\left(\frac{5}{4}\right)^{m}\left|g^{(4)}\left(q_{c}\right)\right|^{-\frac{m}{4}}$ & $\frac{4}{2-r}<d<\frac{8}{2-r}$ & \\
\hline & $(8 \pi J)^{-2}\left(\frac{48}{\pi^{3}}\right)^{\frac{m}{4}} \Gamma\left(\frac{5}{4}\right)^{m}\left|g^{(4)}\left(q_{c}\right)\right|^{-\frac{m}{4}}$ & $d=\frac{8}{2-r}$ & \\
\hline & $\overline{A_{2}}$ & $d>\frac{8}{2-r}$ & \\
\hline \multirow{5}{*}{$\begin{array}{c}m \neq 0 \\
d-m=0 \\
|R|+4 S \neq 0\end{array}$} & $2^{d}(8 \pi J)^{-\frac{d}{2}}\left|g^{(2)}\left(q_{c}\right)\right|^{-\frac{d}{2}}$ & $d<2$ & \multirow{5}{*}{$\alpha_{p}=\frac{d-2}{2}$} \\
\hline & $2^{d}(8 \pi J)^{-1}\left|g^{(2)}\left(q_{c}\right)\right|^{-1}$ & $d=2$ & \\
\hline & $2^{d}(8 \pi J)^{-\frac{d}{2}}\left|g^{(2)}\left(q_{c}\right)\right|^{-\frac{d}{2}}$ & $2<d<4$ & \\
\hline & $2^{d}(8 \pi J)^{-2}\left|g^{(2)}\left(q_{c}\right)\right|^{-2}$ & $d=4$ & \\
\hline & $A_{2}$ & $d>4$ & \\
\hline Caso 4 & $(8 \pi J)^{-\frac{d}{4}}\left(\frac{48}{\pi^{3}}\right)^{\frac{d}{4}} \Gamma\left(\frac{5}{4}\right)^{d}\left|g^{(4)}\left(q_{c}\right)\right|^{-\frac{d}{4}}$ & $d<4$ & \multirow{5}{*}{$\alpha_{p}=\frac{d-4}{4}$} \\
\hline \multirow{4}{*}{$\begin{array}{c}m \neq 0 \\
d-m=0 \\
|R|+4 S=0\end{array}$} & $(8 \pi J)^{-1}\left(\frac{48}{\pi^{3}}\right) \Gamma\left(\frac{5}{4}\right)^{4}\left|g^{(4)}\left(q_{c}\right)\right|^{-1}$ & $d=4$ & \\
\hline & $(8 \pi J)^{-\frac{d}{4}}\left(\frac{48}{\pi^{3}}\right)^{\frac{d}{4}} \Gamma\left(\frac{5}{4}\right)^{d}\left|g^{(4)}\left(q_{c}\right)\right|^{-\frac{d}{4}}$ & $4<d<8$ & \\
\hline & $(8 \pi J)^{-2}\left(\frac{48}{\pi^{3}}\right)^{2} \Gamma\left(\frac{5}{4}\right)^{8}\left|g^{(4)}\left(q_{c}\right)\right|^{-2}$ & $d=8$ & \\
\hline & $A_{2}$ & $d>8$ & \\
\hline Caso 5 & $(8 \pi J)^{-\frac{d}{2}}$ & $d<2$ & \multirow{5}{*}{$\alpha_{p}=\frac{d-2}{2}$} \\
\hline \multirow{2}{*}{$m=0$} & $(8 \pi J)^{-1}$ & $d=2$ & \\
\hline & $(8 \pi J)^{-\frac{d}{2}}$ & $2<d<4$ & \\
\hline \multirow{2}{*}{$d-m \neq 0$} & $(8 \pi J)^{-2}$ & $d=4$ & \\
\hline & $A_{2}$ & $d>4$ & \\
\hline
\end{tabular}

Tabela 2.3: Valores para $F_{p}, \alpha_{p}$ e $g_{p}$. 
A tabela 2.3 é referência para toda a análise que se segue; nela, $g^{(n)}$ é a $n$-ésima derivada da aplicação $g$, definida em (2.28) (não confundir com $g_{p}$ ). Juntamente com a tabela 2.2, nota-se que

$$
\alpha_{p}=\gamma_{p}-1
$$

A forma de $\mathcal{L}[f](s)$ para $s \sim 2 \hat{J}\left(k_{c}\right),(2.33)$, é essencial para determinar o comportamento assintótico de $\psi(t \sim \infty)$ mediante a equação (2.29) (Apêndice A.8). Como a circunstância experimental consiste em, a partir de uma situação desordenada, resfriar o sistema até uma temperatura $T$, três situações distintas são possíveis e são analisadas a seguir. Os detalhes técnicos dos cálculos assintóticos das funções de dois tempos estão no Apêndice A.11.

\subsection{2- Dinâmica supercrítica}

Quando, a partir de uma fase desordenada, o sistema é resfriado até a uma temperatura $T>T_{c}$, a função $\psi$ tem um comportamento exponencial,

$$
\psi(t) \sim e^{t / \tau_{e q}}
$$

onde $\tau_{e q}$ é um tempo característico, estimado no Apêndice A.8 para $T \sim T_{c}^{+}$. Esta peculiaridade é responsável pelo decaimento do sistema a uma situação de equilíbrio em um tempo finito, quando então a autocorrelação,

$$
C\left(t, t^{\prime}\right) \sim C(\tau)=T \int_{\tau}^{\infty} d y f\left(\frac{y}{2}\right) e^{-\frac{y}{2 \tau_{e q}}}
$$

e a função resposta,

$$
R\left(t, t^{\prime}\right) \sim R(\tau)=f\left(\frac{\tau}{2}\right) e^{-\frac{\tau}{2 \tau_{e q}}},
$$

passam a depender somente da diferença de tempo $\tau:=t-t^{\prime}$. Naturalmente, nesta situação, o teorema flutuação-dissipação é válido, fato que é expresso por

$$
X\left(t, t^{\prime}\right) \sim 1
$$

\subsection{3- Dinâmica crítica}

A dinâmica crítica é a única entre as três que requer uma análise mais minunciosa de seu comportamento dinâmico no sentido deste depender da dimensionalidade do sistema. $O$ caso crítico pressupōe a ocorrência de uma transição de fase, o que implica $T_{c} \neq 0$ e, por conseguinte, $d>d_{c}$.

O comportamento assintótico de $\psi$, dado por

$$
\psi(t) \sim \begin{cases}\frac{e^{2 \hat{J}\left(k_{c}\right) t}}{t^{1-\alpha_{p}}}, & d_{c}<d<\bar{d} \\ \frac{e^{2 \hat{J}\left(k_{c}\right) t}}{\ln t} & , \quad d=\bar{d} \\ e^{2 \hat{J}\left(k_{c}\right) t} & , \quad d>\bar{d}\end{cases}
$$


é sensível à dimensão em questão, e a análise deve considerar este fator.

A investigação do comportamento dinâmico crítico das funções de dois tempos leva a uma definição natural de duas escalas de tempo, que não eram observadas no caso supercrítico (lembrando sempre que $\left.1 \ll t^{\prime} \leq t\right)$.

O primeiro regime de tempo, dito estacionário, realiza-se para $1 \sim \tau \ll t^{\prime}$. Neste quadro, o comportamento dinâmico é tipicamente de equilíbrio, e, para qualquer dimensão $d$, tanto a autocorrelação,

$$
C\left(t, t^{\prime}\right) \sim C_{e q, c}(\tau)
$$

quanto a função resposta,

$$
R\left(t, t^{\prime}\right) \sim f\left(\frac{\tau}{2}\right) e^{-\hat{J}\left(k_{c}\right) \tau}
$$

dependem somente da diferença de tempo $\tau$. Aqui, definiu-se

$$
C_{e q, c}(\tau):=T_{c} \int_{\tau}^{\infty} d y f\left(\frac{y}{2}\right) e^{-\hat{J}\left(k_{c}\right) y}
$$

O teorema flutuação - dissipação é naturalmente satisfeito com

$$
X\left(t, t^{\prime}\right) \sim 1
$$

A escolha de um intervalo $\tau$ não muito grande com $1 \sim \tau \ll t^{\prime}$ foi suficiente para impedir que os efeitos do decaimento rumo ao equilíbrio (que evidenciariam o fato do sitema estar, ainda no instante $t^{\prime}$, em uma situação de não - equilíbrio) fossem observados, conferindo ao modelo um caráter de equilíbrio. Logo, tomando o intervalo entre $t^{\prime}$ e $t$ maior, é de se esperar que as propriedades da mecânica estatística de equilíbrio sejam violadas. Este argumento define a segunda escala de tempo, o regime de "aging" (ou envelhecimento), em que $1 \ll \tau \sim t^{\prime}$. Neste regime, é conveniente definir

$$
x:=\frac{t}{t^{\prime}}
$$

esta notação será usada ao longo deste capítulo. A forma assintótica da autocorrelação,

$$
C\left(t, t^{\prime}\right) \sim\left\{\begin{array}{ll}
\frac{2 K_{p} T_{c} 2^{\gamma_{p}}}{\gamma_{p}-1} t^{1-\gamma_{p}} \frac{x^{1-\frac{\gamma_{p}}{2}(x-1)^{1-\gamma_{p}}}}{x+1} & , d_{c}<d<\bar{d} \\
\frac{T_{c} K_{p} 2^{\gamma_{p}}}{\gamma_{p}-1} t^{1-\gamma_{p}}\left[1+\frac{\ln (x+1)}{\ln t^{\prime}}\right]^{-1} \sqrt{1+\frac{\ln x}{\ln t^{\prime}}} & , d=\bar{d} \\
\frac{2^{\gamma_{p}} K_{p} T_{c}}{\gamma_{p}-1} t^{1-\gamma_{p}}(x-1)^{1-\gamma_{p}} & , d>\bar{d}
\end{array},\right.
$$

e a função resposta,

$$
R\left(t, t^{\prime}\right) \sim \begin{cases}2^{\gamma_{p}} K_{p} t^{\prime \gamma_{p}} x^{\frac{1-\alpha_{p}}{2}}(x-1)^{-\gamma_{p}} & , \quad d_{c}<d<\bar{d} \\ 2^{\gamma_{p}} K_{p} t^{\gamma^{-\gamma_{p}}}(x-1)^{-\gamma_{p}} \sqrt{1+\frac{\ln x}{\ln t^{\prime}}} & , \quad d=\bar{d} \\ 2^{\gamma_{p}} K_{p} t^{-\gamma_{p}}(x-1)^{-\gamma_{p}} & , d>\bar{d}\end{cases}
$$


indicam que a invariância por translação temporal é mantida para altas dimensões $(d>\bar{d})$, quando o comportamento assintótico é descrito por uma potência do fator $t^{\prime}(x-1)=t-t^{\prime}$, que é uma função de $\tau$.

O coeficiente de flutuação - dissipação,

$$
X\left(t, t^{\prime}\right) \sim \begin{cases}\frac{\left(\gamma_{p}-1\right)(x+1)^{2}}{\left(\gamma_{p} x+\gamma_{p}-2\right)(x+1)-2(x-1)} & , d_{c}<d<\bar{d} \\ \frac{2\left(\gamma_{p}-1\right)}{\frac{x-1+2\left(\gamma_{p}-1\right) \ln t^{\prime}}{\ln t^{\prime}+\ln (x+1)}-\left(\frac{x-1}{x+1}\right) \frac{\ln t^{\prime}}{\left[\ln t^{\prime}+\ln (x+1)\right]^{2}}} & , d=\bar{d} \\ 1 & , d>\bar{d}\end{cases}
$$

evidencia a violação do teorema flutuação - dissipação para $d_{c}<d \leq \bar{d}$, embora a mesma afirmação não possa ser feita para $d>\bar{d}$. Notar que $\boldsymbol{x} \sim 1$ corresponderia a um caso limite estacionário; de fato, tomar $x \rightarrow 1$ em (2.47) implica $X\left(t, t^{\prime}\right) \sim 1$ nos três casos.

\subsection{4- Dinâmica subcrítica}

A ocorrência de uma transição de fase é, evidentemente, exigida neste caso, o que impõe restrições na dimensionalidade do sistema. Satisfeita esta condição, a dinâmica subcrítica, da mesma forma como na crítica, determina as mesmas escalas de tempo. Em ambos os casos, a função $\psi$ é assintoticamente dada por

$$
\psi(t) \sim \frac{f(t)}{M_{e q}^{4}}
$$

onde o termo

$$
M_{e q}^{2}:=1-\frac{T}{T_{c}}
$$

reforça o caráter subcrítico remetendo à idéia de magnetização do modelo estático.

No regime estacionário de tempo, $1 \sim \tau \ll t^{\prime}$, tanto a autocorrelação,

$$
C\left(t, t^{\prime}\right) \sim M_{e q}^{2}+\left(1-M_{e q}^{2}\right) C_{e q, c}(\tau),
$$

com a função $C_{e q, c}$ definida em (2.42), quanto a função resposta,

$$
R\left(t, t^{\prime}\right) \sim f\left(\frac{\tau}{2}\right) e^{-\hat{J}\left(k_{c}\right) \tau}
$$

possuem características estacionárias, dependendo somente de $\tau$, com o teorema flutuação-dissipação

$$
X\left(t, t^{\prime}\right) \sim 1
$$

sendo válido.

Por outro lado, no regime de tempo de "aging", $1 \ll \tau \sim t^{\prime}$, a autocorrelação, 


$$
C\left(t, t^{\prime}\right) \sim M_{e q}^{2}\left[\frac{4 x}{(x+1)^{2}}\right]^{\frac{\gamma_{p}}{2}}
$$

e a função resposta,

$$
R\left(t, t^{\prime}\right) \sim K_{p} 2^{\gamma_{p} t^{-\gamma_{p}}} x^{\frac{\gamma_{p}}{2}}(x-1)^{-\gamma_{p}}
$$

não são mais invariantes por translação temporal, o que é evidenciado pela dependência tanto de $t^{\prime}$ quanto de $t$. Notar que entre os dois regimes de tempo a autocorrelação assume o valor do plateau

$$
\lim _{\tau \rightarrow \infty} \lim _{t^{\prime} \rightarrow \infty} C\left(t, t^{\prime}\right)=M_{e q}^{2}=1-\frac{T}{T_{c}},
$$

que é equivalente ao parâmetro de ordem de Edwards - Anderson. O cálculo dos dois limites em (2.55) equivale a tomar $\tau \rightarrow \infty \mathrm{em}(2.50)$ ou $x \rightarrow 1 \mathrm{em}(2.53)$. Esta conexão entre os dois regimes permite expressar a autocorrelação como

$$
C\left(t, t^{\prime}\right) \sim\left(1-M_{e q}^{2}\right) C_{e q, c}(\tau)+M_{e q}^{2}\left[\frac{4 x}{(x+1)^{2}}\right]^{\frac{\gamma_{p}}{2}}
$$

que interpola os dois comportamentos dinâmicos.

O teorema flutuação - dissipação,

$$
X\left(t, t^{\prime}\right) \sim \frac{2 T K_{p}}{\gamma_{p} M_{e q}^{2}} t^{\prime^{1-\gamma_{p}}}\left(\frac{x+1}{x-1}\right)^{1+\gamma_{p}},
$$

é violado, sugerindo a existência de temperaturas distintas no sistema.

\section{7- Considerações finais}

Os resultados principais deste capítulo, as dinâmicas supercrítica, crítica e subcrítica, retratam o comportamento das funções de dois tempos (autocorrelação e função resposta) em cada caso. Esta descrição levou em consideração duas escalas de tempo presentes na dinâmica, que discerne o regime estacionário do regime de "aging". O primeiro tem traços tipicamente de um sistema em equilíbrio, apresentando invariância por translação temporal e obediência ao teorema flutuação - dissipação; estas propriedades foram confirmadas nas dinâmicas crítica, subcrítica e também no caso supercrítico, este último por atingir a situação de equilíbrio em tempo finito (lembrar que todas as análises foram feitas para $t^{\prime} \gg 1$ ). A segunda escala de tempo, o regime de "aging", não tem as propriedades supracitadas; as funções de dois tempos não dependem somente da diferença de tempo, $\tau:=t-t^{\prime}$, e o teorema flutuação - dissipação é violado. Estas peculiaridades foram observadas na dinâmica crítica e subcrítica quando $1 \ll \tau \sim t^{\prime}$.

Os resultados listados acima são características gerais da dinâmica dissipativa adotada e já eram esperados, exceto o caso $d>\bar{d}$ na dinâmica crítica. A contribuição do trabalho, em sendo investigar os efeitos de competição na dinâmica de Langevin através de resultados analíticos, deve ter como finalidade comparar os resultados aqui obtidos com a dinâmica de um ferromagneto de curto alcance. Foi eleito como sendo o artigo mais representativo nesse sentido a referência [25], que, expressando de uma forma 
simplória, é um estudo detalhado do caso 5 do presente trabalho. Aparentemente, as únicas discrepâncias (oriundas do efeito de competição) entre os dois trabalhos podem ser resumidas mais em aspectos quantitativos que qualitativos com uma ressalva: não foi encontrado "aging" no caso crítico para $d>\bar{d}$, embora esta diferença não seja proveniente dos efeitos de competição. Ademais, enquanto no modelo ferromagnético de Godrèche e Luck[25] a dimensionalidade crítica restringia-se a $d_{c}=2$, a introdução da competição foi responsável pelo surgimento de um panorama mais rico, onde a dimensão crítica depende do número de direções com interações de primeiros e segundos vizinhos, $m$, do número de direções com interações de primeiros vizinhos, $d-m$, e da razão $S / R$ que fornece a intensidade da competição. A conseqüência disto é refletida nos casos 1 a 4 . Naturalmente, os prefatores da expansão assintótica de $\psi$, o coeficiente de flutuação - dissipação e outros detalhes são dependentes dos parâmetros $m, d, R$ e $S$, mas as conclusões acerca do comportamento dinâmico mantêm-se inalteradas exceto no caso $d>\bar{d}$ da dinâmica crítica, onde foi constatado um regime estacionário neste trabalho, ao passo que em [25] o "aging" foi novamente encontrado.

Como uma observação final, a mecânica estatística de equilíbrio corresponderia em tomar, inicialmente, o limite $t^{\prime} \rightarrow \infty$, tempo "suficiente" para a convergência ao equilíbrio. Notar que, uma vez tomado este limite, para qualquer $t$ (mesmo $t \rightarrow \infty$ ), a razão $\tau / t^{\prime}$ é arbitrariamente pequena ${ }^{9}$, o que corresponde aos casos estacionários estudados, que apresentam características de equilíbrio termodinâmico.

\footnotetext{
${ }^{9} \mathrm{~A}$ ordem da tomada dos limites $t^{\prime} \rightarrow \infty$ e $t \rightarrow \infty$ é essencial para esta conclusão.
} 


\section{Capítulo 3}

\section{Modelo de Viana-Bray com campo aleatório}

\section{1- Introdução}

Do ponto de vista da área de sistemas desordenados, a década de 1970 ficou marcada pelo surgimento de modelos de vidros de spin[49, 50]: as impurezas eram simuladas admitindo a integral de troca como uma variável aletória, numa tentativa de captar as peculiaridades desse sistema e possibilitando, ainda, a caracterização de uma fase vítrea. Nessa mesma década um outro modelo, em que a desordem era atribuída não às interações entre spins, mas ao campo externo, foi proposto. O modelo de Ising na presença de um campo externo aleatório[51] despertou grande interesse; a simplicidade de sua formulação (no sentido de permitir uma vasta análise analítica), sem perder, entretanto, contatos com a parte experimental (o artigo de Fishman e Aharony[52] parece ser a proposta para a realização experimental desse modelo mais conhecida), torna-o bastante atraente. As considerações gerais e históricas acerca deste modelo são apresentadas na seção 3.2.

Embora o cálculo de campo médio, no espírito de Curie-Weiss, para o modelo de Ising com campo aleatório não tenha fornecido resultados excitantes [53, 54], limitando-se apenas a apresentar uma fase ferromagnética e paramagnética, trabalhos mais refinados $[55,56]$, que exploraram terrenos para além do "campo médio usual", indicam a presença de uma fase vitrosa. Na linguagem do método das réplicas (com uma apresentação sucinta na seção 3.3), existe uma região de instabilidade da solução réplica-simétrica. Este assunto é abordado nas seções 3.4 e 3.5, onde se encontra a contribuição do presente trabalho[57]: a investigação sobre a presença (ou ausência) da fase vidros de spin em um ferromagento diluído sob campo externo aleatório. Os resultados são reorganizados e comentados na última seção, 3.6.

\section{2- Modelo de Ising com campo aleatório}

O desenvolvimento da teoria de fenômenos críticos, motivado pelo surgimento do grupo de renormalização em 1971, concebeu modelos de grande importância e interesse no magnetismo. Dentre eles, a introdução de impurezas aleatórias nos modelos de mecânica estatística havia ganhado grande interesse; em particular, o primeiro trabalho em que a aleatoriedade é introduzida no campo externo foi proposto por Imry e Ma[51] em meados da década de 1970.

O interesse por este modelo de Ising na presença de um campo aleatório cuja função de Hamilton é 


$$
H\left(\left\{\sigma_{x}\right\}\right)=-\sum_{x, x^{\prime} \in \Lambda_{N}} J_{x, x^{\prime}} \sigma_{x} \sigma_{x^{\prime}}-\sum_{x \in \Lambda_{N}} H_{x} \sigma_{x}
$$

em que $J_{x, x^{\prime}}=J>0$ para uma interação ferromagnética, cresceu com a conjectura de sua equivalência com um antiferromagneto diluído na presença de um campo externo uniforme[52], e esta idéia ganhou força com o trabalho de Cardy[58], que mostrou que os comportamentos estáticos críticos de ambos modelos eram idênticos; trabalhar com um antiferromagneto diluído submetido a um campo uniforme é particularmente mais atraente do ponto de vista experimental. Atualmente, há uma significativa diversidade de experimentos relacionados ao modelo de Ising com campo aleatório[59]. A equivalência, entretanto, não é assegurada nos domínios da dinâmica.

A questão da dimensão crítica, que era um problema em aberto que atraía muito interesse, já havia sido investigado por Imry e $\mathrm{Ma}[51]$ para interações ferromagnéticas de curto alcance $\left(J_{x, x^{\prime}}=J>0\right.$ para $\left\|x-x^{\prime}\right\|_{1}=1$, e $J_{x, x^{\prime}}=0$ caso contrário) e campo aleatório descorrelacionado e de média nula,

$$
\mathbb{E}\left\{H_{x}\right\}=0 \quad \text { e } \quad \mathbb{E}\left\{H_{x} H_{x^{\prime}}\right\}=H_{R}^{2} \delta_{x, x^{\prime}},
$$

onde a esperança é tomada sobre a desordem. É importante destacar que se o campo aleatório for suficientemente grande o sistema é desordenado mesmo para temperaturas baixas[60]. O caso de interesse é um campo aleatório suficientemente pequeno a ponto de evitar esta situação. Com argumentos heurísticos, baseados na estimativa energética de formação de ilhas ordenadas no sistema, Imry e Ma concluíram que para $d \leq 2$ a fase ferromagnética é instável no estado fundamental. A dimensão crítica $d_{c}$ foi rigorosamente estabelecida como sendo $d_{c}=2$ por Imbrie[61] em meados da década de 1980 para integrais de troca de curto alcance.

A versão de campo médio para o modelo de Ising com campo aleatório foi estudada logo após sua aparição. Foram consideradas as distribuições de probabilidade tipo Gauss[53, 54] e bimodal[54] para o campo aleatório. Em ambos casos, encontrou-se um diagrama de fases sem muito interesse, contendo somente as fases ferromagnética e paramagnética. A diferença marcante entre as duas distribuições era a presença de um ponto tricrítico no caso bimodal, ao passo que para a distribuição de Gauss as fases ordenada e desordenada eram separadas apenas por uma linha crítica de segunda ordem. Há, porém, uma controvérsia quanto à natureza da transição em sistemas de curto alcance para campos bimodais, apontada por trabalhos computacionais[62].

Trabalhos de revisão acerca dos assuntos abordados acima podem ser encontrados em [63] e [62].

A versão de campo médio, à la Curie-Weiss, do modelo de Ising com campo aleatório, embora solúvel de forma rigorosa[64] pelo método de van Hemmen[65, 66] e também pelo truque de réplicas[53] (constituindo, pois, um ótimo laboratório para testar este método), não apresenta, como foi dito, resultados interessantes. Este fato, no entanto, não restringe um ferromagneto sob campo aleatório a exibir somente uma fase ferromagnética e uma outra paramagnética. $O$ estado fundamental desse modelo na rede de Bethe é bastante rico[67]; analisando através de um campo médio mais refinado, uma fase vidros de spin é encontrada por de Almeida e Bruinsma[55] em um antiferromagneto diluído sob ação de um campo externo, e por Pastor et al. [56] na rede de Bethe. A fase vítrea é esperada em dimensões finitas para o modelo de Ising com campo aleatório, o que além de ser um resultado interessante por si, viola a hipótese de redução dimensional[68].

\section{3- Truque de réplicas}

A identidade

$$
\ln x=\lim _{n \rightarrow 0} \frac{x^{n}-1}{n}=\lim _{n \rightarrow 0} \frac{1}{n} \ln x^{n}, \quad x \in \mathbb{R}_{+},
$$


que é de fácil verificação e conhecida há muito tempo, popularizou-se em física da matéria condensada de forma definitiva a partir do trabalho pioneiro de Edwards e Anderson[49], que introduziu um modelo para vidros de spin de curto alcance; a sua versão de campo médio foi proposta logo em seguida por Sherrington e Kirkpatrick[50].

O problema encontrado nestes dois modelos é oriundo da existência de duas escalas de tempo no modelo. Nestes sistemas, além da termalização dos spins, a integral de troca é uma variável aleatória cuja variação com o tempo é um fato que não pode ser ignorado. A dificuldade surge quando a ordem de grandeza do tempo de termalização da integral de troca é bem superor à da termalização dos spins. Operacionalmente, isto significa que a energia livre por sítio, em uma rede finita $\Lambda_{N}$ de $N$ sítios, a ser calculada é dada por

$$
f_{\Lambda}(\beta)=-\frac{1}{\beta N} \mathbb{E}_{J}\left\{\ln Z_{\Lambda}\left(\beta,\left\{J_{x, x^{\prime}}\right\}\right)\right\} .
$$

Esta é a situação do limite de desordem temperada ("quenched"). O outro extremo, o limite de desordem recozida ("annealed"), realiza-se na situação em que o tempo de termalização dos spins é bem maior que o das integrais de troca; este caso, embora de simples tratamento analítico, é de menor interesse físico, visto que o outro, além de representar um desafio teórico pelos obstáculos técnicos envolvidos, encontrou em materiais tão familiares como vidros, por exemplo, a sua imagem experimental.

O empecilho técnico imposto pela equação (3.4) no cálculo da esperança de uma função logarítmica para $x \in \mathbb{R}_{+}$resultou na ousada decisão de recorrer à identidade (3.3), fornecendo

$$
f(\beta)=\lim _{N \rightarrow \infty} f_{\Lambda}(\beta)=-\lim _{N \rightarrow \infty} \lim _{n \rightarrow 0} \frac{1}{\beta n N} \ln \mathbb{E}_{J}\left\{Z_{\Lambda}^{n}(\beta)\right\},
$$

para contornar o problema da determinação da energia livre $f$. A esperança a ser tomada seria, então, sobre um produto independente de $n$ cópias (réplicas) da função de partição.

O truque de réplicas, apesar de seu sucesso (no sentido de haver conseguido grande popularidade, fato este comprovado nas inúmeras implementações), enfrentou críticas, sendo as principais delas de natureza matemática. Destacar-se-ão, aqui, dois problemas:

(i) Troca dos limites $N \rightarrow \infty$ e $n \rightarrow 0$ em (3.5)

(ii) Continuação analítica de $n$.

O significado dessas questões será esclarecido mais adiante, e a explicação será baseada através de um exemplo, o paradigma de um sistema desordenado: o modelo de Sherrington e Kirkpatrick, a versão de campo médio à la Curie-Weiss para vidros de spin.

Em geral, o desenvolvimento de (3.5) leva a

$$
f(\beta)=-\lim _{N \rightarrow \infty} \lim _{n \rightarrow 0} \frac{1}{\beta n N} \ln \int e^{-N \psi\left(\left\{q_{x}\right\}\right)} \prod_{x \in X} d q_{x}
$$

para algum conjunto de índices $X$. Este conjunto de índices é fruto da tomada da esperança em (3.5), que tem como efeito acoplar as $n$ réplicas inicialmente independentes. $O$ campo $q_{x}$, entendido como parâmetro de ordem, aparece em apenas dois tipos no caso do modelo de Sherrington - Kirkpatrick, $q_{\alpha}$ e $q_{\alpha \beta}$, com $\alpha, \beta \in\{1, \cdots, n\}$; de forma geral, tem-se

$$
q_{\alpha_{1} \cdots \alpha_{r}}:=\mathbb{E}\left\{\left\langle\sigma_{1} \cdots \sigma_{r}\right\rangle\right\},
$$

onde $\langle\cdot\rangle$ indica média térmica. O parâmetro $q_{\alpha}=m_{\alpha}$ é associado à magnetização, e $q_{E A}:=q_{\alpha \beta}$ é conhecido como o parâmetro de ordem de Edwards-Anderson. Notar que se a comutação entre os dois 
limites presentes em (3.6) for possível, o problema haverá de sugerir sua resolução pelo método do ponto de sela, quando então o cálculo da energia livre é reduzido a

$$
f(\beta)=\lim _{n \rightarrow 0} \frac{1}{\beta n} \inf _{\substack{\left\{q_{x}\right\} \\ x \in X}} \psi\left(\left\{q_{x}\right\}\right)
$$

e um conjunto de equações de estado

$$
\frac{\partial \psi\left(\left\{q_{x}\right\}\right)}{\partial q_{x}}=0
$$

que é a extremização de $\psi$, condição necessária para a determinação de seu ínfimo. O problema da comutação entre os limites termodinâmico $(N \rightarrow \infty)$ e $n \rightarrow 0$ (crítica (i)) foi abordado por van Hemmen e Palmer. Estes autores estudaram a legitimidade dessa operação no caso do modelo de Sherrington e Kirkpatrick[69], além de introduzirem condições favoráveis para possibilitar a troca no caso de sistemas de curto alcance[70]. (3.9).

Admitida a possibilidade de trocar $N \rightarrow \infty$ e $n \rightarrow 0$, o problema resume-se nas equações (3.8) e

O conjunto $\left\{q_{x}\right\}$ que minimiza $\psi$, porém, não é tecnicamente de fácil acesso; o procedimento usual para tratar o problema é admitir um Ansatz para a solução. Inicialmente, decompõe-se $\left\{q_{x}\right\}$ em subconjuntos $\left\{q_{x}\right\}=\cup_{i}\left\{q_{x_{i}}\right\}$; esta decomposição, que forma uma classe de equivalência, é de tal sorte que o número de índices em $x_{i}$ é diferente do de $x_{j}$ se, e somente se, $i \neq j$. Exemplificando, $\left\{q_{\alpha}\right\}$ e $\left\{q_{\alpha \beta}\right\}$ são duas dessas classes distintas.

O Ansatz para a solução prescreve admitir que todos os elementos para cada um dos subconjuntos $\left\{q_{x_{i}}\right\}$ sejam iguais, $q_{x_{i}}=q_{i}$. Este procedimento constitui a solução réplica-simétrica. A validade desta hipótese é assegurada se esta solução for, de fato, o ínfimo de $\psi$, o que pode ser verificado pela análise de estabilidade via matriz de Hesse,

$$
\frac{\partial^{2} \psi\left(\left\{q_{x}\right\}\right)}{\partial q_{x} \partial q_{y}}
$$

examinando a positividade de seus autovalores. Esta análise foi realizada pela primeira vez em [71] para o modelo de Sherrington-Kirkpatrick, onde foi encontrada uma região de instabilidade da solução réplicasimétrica; em outros termos, tomar todos parâmetros de ordem de classes de equivalência iguais não correspondia a um mínimo de $\psi$, e a simetria de permutação entre as réplicas deveria ser quebrada. A sugestão heurística de Parisi[72] de como esta quebra deveria ser feita veio a resolver esta questão. Segundo esta proposta, a quebra poderia ser feita em etapas, e em cada passo a assimetria sendo incrementada. Esta idéia veio a fornecer uma solução para o modelo de Sherrington-Kirkpatrick, que exigia um número infinito de quebras de simetria. Estes passos eram responsáveis pela profusão de um número grande, que crescia exponencialmente com o número de sítios da rede, de estados metaestáveis, ultrametricamente organizados[73], correspondendo a mínimos locais da energia livre.

A solução de Parisi, tal como o truque de réplicas, parte do pressuposto de que $n$ é um número inteiro (eventualmente "muito grande"), com os cálculos sendo desenvolvidos, até certo ponto, sob esta hipótese; a sua continuação analítica (exigida por (3.3) ou (3.5)) para um número real, com a intenção de levá-lo posteriormente a zero, causa estranheza, e atitudes questionando a legitimidade desta operação, que constituem a base da crítica (ii), são inevitáveis.

Existem várias publicações de revisão acerca dos assuntos acima, sendo [74] e [75] os trabalhos provavelmente mais conhecidos.

A solução heurística de Parisi só veio a ganhar credibilidade dentro do contexto da física-matemática em 2003 com o trabalho rigoroso de Talagrand[76], que demonstrou a legitimidade de seu resultado. 


\section{4- Modelo de Viana-Bray com campo aleatório}

\subsection{1- Introdução}

Alternativamente à forma tradicional de incorporar a conectividade como parâmetro numa análise de campo médio, através da construção de uma árvore de Cayley (ou também através de uma rede de Bethe), Viana e Bray[77] introduziram um modelo capaz de admitir aleatoriedade nas ligações entre spins, e a grandeza em questão é a conectividade média. Este modelo foi inicialmente estudado nas proximidades da temperatura de transição[77]; o comportamento a baixas temperaturas foi tratado alguns anos mais tarde por Kanter e Sompolinsky[78] e Mézard e Parisi[79]. A análise de estabilidade das fases no estado fundamental foi realizada em $[80,81]$ no limite de percolação. O estudo de um modelo com conectividade finita e na presença de um campo externo parece se resumir ao artigo de Bruinsma[67], não havendo indícios de trabalho similar no modelo de Viana-Bray. Para conciliar a conectividade (média) $c$ com a assinatura de um campo médio tipo Curie-Weiss, a integral de troca $J_{x, x^{\prime}}$, entre spins de Ising localizados nos sítios $x$ e $x^{\prime}$, é considerada como uma variável aleatória que assume, com probabilidade $1-c / N$, valor zero, e assume um valor finito com probabilidade $c / N$, onde $N$ é o número de sítios de uma rede finita $\Lambda_{N}$. A função de Hamilton do modelo de Viana-Bray é, pois,

$$
H\left(\left\{\sigma_{x}\right\}\right)=-\sum_{\substack{x, x^{\prime} \in \Lambda_{N} \\ x \neq x^{\prime}}} J_{x, x^{\prime}} \sigma_{x} \sigma_{x^{\prime}}-\sum_{x \in \Lambda_{N}} H_{x} \sigma_{x},
$$

com $\sigma_{x} \in\{-1,1\}, \forall x \in \Lambda_{N}$, e a função de distribuição das variáveis aleatórias $\left\{J_{x, x^{\prime}}\right\}$ dada por

$$
p_{J}\left(J_{x, x^{\prime}}\right)=\left(1-\frac{c}{N}\right) \delta\left(J_{x, x^{\prime}}\right)+\frac{c}{N} \rho\left(J_{x, x^{\prime}}\right) .
$$

A função de distribuição $\rho$ das ligações é arbitrária. No presente trabalho, toma-se

$$
\rho\left(J_{x, x^{\prime}}\right)=\delta\left(J_{x, x^{\prime}}-J_{0}\right)
$$

com $J_{0}>0$, para mimetizar um ferromagneto. Indo um passo adiante em relação aos trabalhos já publicados $[78,79,80]$, será introduzido um campo externo aletório que obedece a uma distribuição discreta bimodal,

$$
p_{H}\left(H_{x}\right)=\frac{1}{2} \delta\left(H_{x}-H_{R}\right)+\frac{1}{2} \delta\left(H_{x}+H_{R}\right),
$$

ao sistema.

Os ingredientes acima permitem determinar a energia livre (estacionária) do modelo. Para tal, recorrese ao truque de réplicas,

$$
f(\beta)=\mathbb{E}_{J}\left\{\mathbb{E}_{H}\left\{\ln Z\left(\beta,\left\{J_{x, x^{\prime}}\right\},\left\{H_{x}\right\}\right)\right\}\right\}=\lim _{n \rightarrow 0} \frac{1}{n} \ln \mathbb{E}_{J}\left\{\mathbb{E}_{H}\left\{Z^{n}\left(\beta,\left\{J_{x, x^{\prime}}\right\},\left\{H_{x}\right\}\right)\right\}\right\} .
$$

Da comutação entre os limites $\lim _{N \rightarrow \infty}$ e $\lim _{n \rightarrow 0}$, chega-se a

$$
f(\beta)=-\lim _{n \rightarrow 0} \lim _{N \rightarrow \infty} \frac{1}{\beta n N} \ln \exp \left[-N \psi\left(\left\{q_{\alpha_{1} \cdots \alpha_{r}}\right\}\right)\right]
$$

onde 


$$
\begin{aligned}
\psi\left(\left\{q_{\alpha_{1} \cdots \alpha_{r}}\right\}\right):= & \frac{c}{2}+\frac{c}{2} \sum_{r=0}^{n} \sum_{\left(\alpha_{1}, \cdots, \alpha_{r}\right)} b_{r} q_{\alpha_{1} \cdots \alpha_{r}}^{2}-\ln \operatorname{Tr}_{\left\{\sigma_{\alpha}\right\}} \exp \left[c \sum_{r=0}^{n} \sum_{\left(\alpha_{1}, \cdots, \alpha_{r}\right)} b_{r} q_{\alpha_{1} \cdots \alpha_{r}} \sigma_{\alpha_{1}} \cdots \sigma_{\alpha_{r}}+\right. \\
& \left.+\ln \int_{\mathbb{R}} d H p_{H}(H) \exp \left(\beta H \sum_{\alpha=1}^{n} \sigma_{\alpha}\right)\right]
\end{aligned}
$$

onde $\sum_{\left(\alpha_{1}, \cdots, \alpha_{r}\right)}$ indica uma soma nos índices $1 \leq \alpha_{1}<\cdots<\alpha_{r} \leq n$.

Deve-se ter sempre em mente que o produto interno

$$
\langle u, v\rangle_{n}:=\operatorname{Tr}_{\left\{\sigma_{\alpha}\right\}}\left[u\left(\left\{\sigma_{\alpha}\right\}\right) v\left(\left\{\sigma_{\alpha}\right\}\right)\right], \quad u, v \in \mathfrak{H}^{(n)},
$$

define um espaço de Hilbert $\mathfrak{H}^{(n)}$ de dimensão finita $\left(\operatorname{dim} \mathfrak{H}^{(n)}=2^{n}\right)$.

A equação (3.16) expressa a tradicional aplicação do método do ponto de sela na determinação da energia livre via truque de réplicas. Em concordância com este método, o argumento de sua exponencial deve conter o ínfimo da função $\psi$ dentre todos os valores de $\left\{q_{\alpha_{1} \cdots \alpha_{r}}\right\}$. A extremização de $\psi$,

$$
\frac{\partial \psi\left(\left\{q_{\alpha_{1} \cdots \boldsymbol{\alpha}_{r}}\right\}\right)}{\partial q_{\alpha_{1} \cdots \boldsymbol{\alpha}_{r}}}=0
$$

que é um sistema de $\left(\begin{array}{l}n \\ r\end{array}\right)$ equações, pode ser condensada na equação

$$
q_{\alpha_{1} \cdots \alpha_{r}}=\frac{1}{Z_{n G}} \operatorname{Tr}_{\left\{\sigma_{\alpha}\right\}} \sigma_{\alpha_{1}} \cdots \sigma_{\alpha_{r}} \exp \left[G\left(\left\{\sigma_{\alpha}\right\}\right)+\ln \int_{\mathbb{R}} d H p_{H}(H) \exp \left(\beta H \sum_{\alpha=1}^{n} \sigma_{\alpha}\right)\right]
$$

onde

$$
Z_{n G}:=\int_{\mathbb{R}} d H p_{H}(H) \operatorname{Tr}_{\left\{\sigma_{\alpha}\right\}} \exp \left[G\left(\left\{\sigma_{\alpha}\right\}\right)+\beta H \sum_{\alpha=1}^{n} \sigma_{\alpha}\right]
$$

com

$$
G\left(\left\{\sigma_{\alpha}\right\}\right):=c \sum_{r=0}^{n} \sum_{\left(\alpha_{1}, \cdots, \alpha_{r}\right)} b_{r} q_{\alpha_{1} \cdots \alpha_{r}} \sigma_{\alpha_{1}} \cdots \sigma_{\alpha_{r}}
$$

que é um parâmetro de ordem global e

$$
b_{r}:=\int_{\mathbb{R}} d J \rho(J) \cosh ^{n}(\beta J) \tanh ^{r}(\beta J)
$$

A energia livre estacionária é dada por 


$$
\begin{aligned}
f(\beta)= & \frac{1}{\beta} \lim _{n \rightarrow 0} \frac{1}{n}\left\{\frac{c}{2}+\frac{c}{2} \sum_{r=0}^{n} \sum_{\left(\alpha_{1}, \cdots, \alpha_{r}\right)} b_{r} q_{\alpha_{1}, \cdots, \alpha_{r}}^{2}-\ln \int_{\mathbb{R}} d H p_{H}(H) \times\right. \\
& \left.\times \operatorname{Tr}_{\left\{\sigma_{\alpha}\right\}} \exp \left[G\left(\left\{\sigma_{\alpha}\right\}\right)+\beta H \sum_{\alpha=1}^{n} \sigma_{\alpha}\right]\right\}
\end{aligned}
$$

se os valores assumidos por $\left\{q_{\alpha_{1} \cdots \alpha_{r}}\right\}$ obedecem à condição de extremo (3.20).

Os cálculos acima estão detalhados no Apêndice B.1.

\subsection{2- Solução réplica-simétrica}

A extremização da energia livre requer a determinação do conjunto $\left\{q_{\alpha_{1} \cdots \alpha_{r}}\right\}$, obtido através da equação (3.20). O obstáculo técnico imposto por esta equação sugere a procura de soluções simples, provavelmente parciais, dedutíveis sem grandes esforços. O efeito do cálculo da esperança de $Z^{n}$ (que inicialmente eram cópias independentes de $n$ sistemas) é visível em (3.17), onde as $n$ cópias do sistema coexistem dependentemente, indicando que as réplicas passaram a ser acopladas. A busca por uma solução réplica-simétrica será feita com um passo intermediário, com a introdução de um campo efetivo $h$, que atuará da mesma forma, com a mesma distribuição de probabilidades $P$, em todas as réplicas. Inspirado na forma da equação (3.20), o campo efetivo será tal que

$$
q_{\alpha_{1} \cdots \alpha_{r}}=\int_{\mathbb{R}} d h P(h) \frac{\operatorname{Tr}_{\left\{\sigma_{\alpha}\right\}} \sigma_{\alpha_{1}} \cdots \sigma_{\alpha_{r}} e^{\beta h \sum_{\alpha=1}^{n} \sigma_{\alpha}}}{\operatorname{Tr}_{\left\{\sigma_{\alpha}\right\}} e^{\beta h \sum_{\alpha=1}^{n} \sigma_{\alpha}}}
$$

Um cálculo simples mostra que

$$
q_{\alpha_{1} \cdots \alpha_{r}}=\int_{\mathbb{R}} d h P(h) \tanh ^{r}(\beta h)
$$

e uma comparação entre (3.20) e (3.25) relaciona as duas grandezas de interesse, $P$ e $G$,

$$
\int_{\mathbb{R}} \frac{d h P(h) e^{\beta h \sum_{\alpha=1}^{n} \sigma_{\alpha}}}{2^{n} \cosh ^{n}(\beta h)}=\frac{1}{Z_{n G}} \exp \left[G\left(\left\{\sigma_{\alpha}\right\}\right)+\ln \int_{\mathbb{R}} d H p_{H}(H) \exp \left(\beta H \sum_{\alpha=1}^{n} \sigma_{\alpha}\right)\right] .
$$

O parâmetro de ordem global, ao ser explicitado em função da distribuição $P$,

$$
\begin{aligned}
G\left(\left\{\sigma_{\alpha}\right\}\right)= & c \int_{\mathbb{R}} d J \rho(J) \int_{\mathbb{R}} d h P(h) \cosh ^{n}(\beta J) \times \\
& \times \exp \left\{\sum_{\alpha=1}^{n} \sigma_{\alpha} \tanh ^{-1}[\tanh (\beta J) \tanh (\beta h)]+\frac{n}{2} \ln \left[1-\tanh ^{2}(\beta J) \tanh ^{2}(\beta h)\right]\right\},
\end{aligned}
$$

revela que, dentro do contexto dessa solução réplica-simétrica, sua dependência com os spins dá-se de uma forma bem peculiar, a saber, $G$ depende apenas da soma deles em todas as réplicas. De (3.28), definindo $\hat{\sigma}:=\sum_{\alpha=1}^{n} \sigma_{\alpha}$, é evidente que 


$$
G\left(\left\{\sigma_{\alpha}\right\}\right)=G(\hat{\sigma})
$$

Agora, para se tomar o limite $n \rightarrow 0$, deve-se primeiramente alargar o espaço de Hilbert $\mathfrak{H}^{(n)}$ (de dimensão finita) para um espaço de Hilbert $\mathfrak{H}$ de dimensão infinita. Em $\mathfrak{H}$, o produto interno

$$
\langle u, v\rangle_{\infty}:=\int_{\mathbb{R}} u^{*}(y) v(y) d y, \quad u, v \in \mathfrak{H},
$$

pressupõe que seus elementos sejam integráveis.

Nesta situação, impõe-se a continuação analítica de $\hat{\sigma} \in[-n, n] \subset \mathbb{Z}$ para $\hat{\sigma} \in \mathbb{C}$. Escolhendo então $\hat{\sigma}=i y / \beta$, com $y \in \mathbb{R}$, a equação (3.27), tomando o limite $n \rightarrow 0$, assume a forma (Apêndice B.3)

$$
P(h)=\int_{\mathbb{R}} d H p_{H}(H) \int_{\mathbb{R}} d y \exp \left[-i y(h-H)+G\left(\frac{i y}{\beta}\right)-c\right] .
$$

A continuação analítica em $\hat{\sigma}$ permitiu introduzir uma transformada de Fourier que possibilitou escrever a dependência funcional de $P$ com $G$. ${ }^{10}$

Uma vez que nas mesmas condições (extensão do espaço de Hilbert e continuação analítica de $\hat{\sigma}$ ) a equação (3.28) adquire a forma

$$
G\left(\frac{i y}{\beta}\right)=c \int_{\mathbb{R}} d J \rho(J) \int_{\mathbb{R}} d h P(h) \exp \left\{\frac{i y}{\beta} \tanh ^{-1}[\tanh (\beta J) \tanh (\beta h)]\right\},
$$

a equação (3.31) passa a ser escrita como uma equação integral não-linear,

$$
\begin{aligned}
P(h)= & \int_{\mathbb{R}} d H p_{H}(H) \int_{\mathbb{R}} d y \exp \left\{-c-i y(h-H)+c \int_{\mathbb{R}} d J \rho(J) \int_{\mathbb{R}} d x P(x) \times\right. \\
& \times \exp \left[\frac{i y}{\beta} \tanh ^{-1}[\tanh (\beta J) \tanh (\beta x)]\right\},
\end{aligned}
$$

e o problema da extremização de $\psi((3.17)$ e (3.19)) transformou-se na determinação da função distribuição $P$ na equação acima na solução réplica-simétrica.

Cabe observar que a procura pela função distribuição $P$ é feita após o limite $n \rightarrow 0$ ter sido tomado, o que não ocorre no caso do modelo de Sherrington-Kirkpatrick. A implicação desta inversão é estar em busca de soluções em um espaço (infinitamente) maior que o apresentado pelo modelo inicial.

O primeiro interesse deste trabalho é a investigação do comportamento crítico a baixas temperaturas; em particular, o estado fundamental $(\beta \rightarrow \infty)$ é investigado. A resolução da equação (3.33) foi obtida somente para uma classe de soluções onde o campo aleatório $H_{R}$ é um múltiplo da intensidade ferromagnética $J_{0}$, que é referente a uma subclasse das distribuições discretas de $P$, que então pode ser representada por

$$
P(h)=\sum_{k \in \mathbb{Z}} a_{k} \delta\left(h-k J_{0}\right)
$$

\footnotetext{
${ }^{10}$ A energia livre estacionária pode ser expressa em termos de $P$, como pode ser visto no Apêndice B.4.
} 
Ainda sobre a condição $m:=H_{R} / J_{0} \in \mathbb{Z}_{+}$, observa-se que, a partir de (3.13) e (3.34),

$$
\begin{aligned}
\lim _{\beta \rightarrow \infty} G\left(\frac{i x}{\beta}\right) & =c e^{-i x J_{0}} \int_{\left(-\infty,-J_{0}\right]} d h P(h)+c \int_{\left(-J_{0}, J_{0}\right)} d h P(h) e^{i x \operatorname{sign}(h) \min \left\{J_{0},|h|\right\}}+c e^{i x J_{0}} \int_{\left[J_{0}, \infty\right)} d h P(h) \\
& =c a_{0}+c \sum_{k=1}^{\infty} a_{k} e^{i x J_{0}}+c \sum_{k=-\infty}^{-1} a_{k} e^{-i x J_{0}} \\
& =A+B e^{i x J_{0}}+C e^{-i x J_{0}}
\end{aligned}
$$

onde

$$
A:=c a_{0}, \quad B:=c \sum_{k=1}^{\infty} a_{k} \quad \text { e } \quad C:=c \sum_{k=-\infty}^{-1} a_{k} .
$$

Pode-se mostrar (Apêndice B.6) que

$$
a_{k}=\frac{e^{A-c}}{2}\left[\left(\frac{B}{C}\right)^{\frac{k+m}{2}} I_{k+m}(2 \sqrt{B C})+\left(\frac{B}{C}\right)^{\frac{k-m}{2}} I_{k-m}(2 \sqrt{B C})\right]
$$

além de

$$
A=\frac{c e^{A-c}}{2}\left[\left(\frac{B}{C}\right)^{\frac{m}{2}}+\left(\frac{B}{C}\right)^{-\frac{m}{2}}\right] I_{m}(2 \sqrt{B C})
$$

e

$$
\begin{aligned}
B= & e^{B+C}-e^{C} \delta_{m, 0}-C\left(\frac{C}{B}\right)^{\frac{m-1}{2}} \int_{0}^{1} d u e^{C u}(1-u)^{\frac{m-1}{2}} I_{m-1}(2 \sqrt{B C(1-u)})+ \\
& +B\left(\frac{B}{C}\right)^{\frac{m}{2}} \int_{0}^{1} d u e^{B u}(1-u)^{\frac{m}{2}} I_{m}(2 \sqrt{B C(1-u)})
\end{aligned}
$$

A terceira equação necessária para fixar $A, B$ e $C$ pode ser dada pela condição

$$
\lim _{\beta \rightarrow \infty} G\left(\frac{i 0}{\beta}\right)=c=A+B+C,
$$

que é facilmente dedutível de (3.32) e (3.35).

\section{5- Análise de estabilidade}

A determinação da energia livre estacionária, convertida na busca do ínfimo de $\psi\left(\left\{q_{\alpha_{1} \cdots \alpha_{r}}\right\}\right),(3.17)$, através de uma aplicação do método do ponto de sela, passou a estar sujeita à resolução da equação 
(3.19). A fim de tentar contornar os meandros técnicos envolvidos, procura-se sugerir soluções para esta equação, e uma proposta simples é a solução réplica-simétrica. Esta solução, porém, é somente um extremizador de $\psi$. A condição que legitimiza a raíz encontrada como sendo um minimizador é a análise da estabilidade da solução. Optou-se em prosseguir o estudo com o parâmetro de ordem global $G$ (ao invés da distribuição $P$ ), verificando se ele minimiza o funcional

$$
\begin{aligned}
\psi[G]= & \frac{c}{2}+\frac{1}{2 c 2^{2 n}} \sum_{r=0}^{n} \sum_{\left(\alpha_{1}, \cdots, \alpha_{r}\right)} \frac{1}{b_{r}} \operatorname{Tr}_{\left\{\sigma_{\alpha}\right\}} \operatorname{Tr}_{\left\{\tau_{\alpha}\right\}} G\left(\left\{\sigma_{\alpha}\right\}\right) G\left(\left\{\tau_{\alpha}\right\}\right) \sigma_{\alpha_{1}} \cdots \sigma_{\alpha_{r}} \tau_{\alpha_{1}} \cdots \tau_{\alpha_{r}}- \\
& -\ln \operatorname{Tr}_{\left\{\sigma_{\alpha}\right\}} \int_{\mathbb{R}} d H p_{H}(H) e^{G\left(\left\{\sigma_{\alpha}\right\}\right)+\beta H \sum_{\alpha=1}^{n} \sigma_{\alpha}}
\end{aligned}
$$

obtido de (3.17) e (3.22).

Este problema conduz à investigação da estabilidade da solução $G$ na matriz de Hesse,

$$
\frac{\delta^{2} \psi[G]}{\delta G\left(\left\{\sigma_{\alpha}\right\}\right) \delta G\left(\left\{\tau_{\alpha}\right\}\right)}
$$

através do comportamento de seus autovalores, a serem encontrados na equação secular

$$
\operatorname{Tr}_{\left\{\tau_{\alpha}\right\}} \frac{\delta^{2} \psi[G]}{\delta G\left(\left\{\sigma_{\alpha}\right\}\right) \delta G\left(\left\{\tau_{\alpha}\right\}\right)} \varphi\left(\left\{\tau_{\alpha}\right\}\right)=\lambda \varphi\left(\left\{\sigma_{\alpha}\right\}\right)
$$

ou (Apêndice B.7)

$$
\begin{aligned}
& \lambda \operatorname{Tr}_{\left\{\sigma_{\alpha}\right\}} \int_{\mathbb{R}} d J \rho(J) e^{\beta J \sum_{\alpha=1}^{n} \sigma_{\alpha} s_{\alpha}} \varphi\left(\left\{\sigma_{\alpha}\right\}\right)= \\
& =\frac{1}{c} \varphi\left(\left\{s_{\alpha}\right\}\right)+\frac{G(\hat{s})}{c} \int_{\mathbb{R}} d H p_{H}(H) \operatorname{Tr}_{\left\{\sigma_{\alpha}\right\}} \frac{e^{G(\hat{\sigma})+\beta H} \sum_{\alpha=1}^{n} \sigma_{\alpha} \varphi\left(\left\{\sigma_{\alpha}\right\}\right)}{Z_{n G}}- \\
& \quad-\int_{\mathbb{R}} d H p_{H}(H) \operatorname{Tr}_{\left\{\sigma_{\alpha}\right\}} e^{G(\hat{\sigma})+\beta H \sum_{\alpha=1}^{n} \sigma_{\alpha}} \int_{\mathbb{R}} d J \rho(J) e^{\beta J \sum_{\alpha=1}^{n} \sigma_{\alpha} s_{\alpha}} \frac{\varphi\left(\left\{\sigma_{\alpha}\right\}\right)}{Z_{n G}},
\end{aligned}
$$

onde a notação (3.29) está sendo usada. É conveniente recorer, agora, ao seguinte resultado devido a Mottishaw e de Dominicis[80],

Teorema 3.1 (Mottishaw - de Dominicis): Na solução réplica-simétrica, com a equação secular dada por (3.44), o espaço dos autovetores $\varphi\left(\left\{\sigma_{\alpha}\right\}\right)$ é varrido pelo conjunto de $2^{n}$ funçôes de duas variáveis $\varphi_{\left\{\mu_{\alpha}\right\}}^{\prime}\left(\hat{\sigma}, q_{\sigma \mu}\right) ;$ simbolicamente,

$$
\varphi\left(\left\{\sigma_{\alpha}\right\}\right) \rightarrow \varphi_{\left\{\mu_{\alpha}\right\}}^{\prime}\left(\hat{\sigma}, q_{\sigma_{\mu}}\right),
$$

onde $q_{\sigma \mu}:=\sum_{\alpha=1}^{n} \sigma_{\alpha} \mu_{\alpha}$ e $\left\{\mu_{\alpha}\right\}$ é um conjunto de vetores que rotula o autovetor.

A prova do teorema encontra-se no Apêndice B.8; fazendo uso dele, a resolução do problema de autovalores é feita inicialmente transformando-a na seguinte equação integral linear 


$$
\begin{aligned}
& \lambda \int_{\mathbb{R}} d y_{1} \int_{\mathbb{R}} d y_{2} \tilde{\chi}\left(i y_{1}, i y_{2}\right) \int_{\mathbb{R}} d h_{1} \int_{\mathbb{R}} d h_{2} e^{-i y_{1} h_{1}-i y_{2} h_{2}} \int_{\mathbb{R}} d J \rho(J) \exp \left[\frac{i \hat{\mu}}{2} \max \left\{\left|h_{1}\right|,|J|\right\}-\right. \\
& \left.-\frac{i \hat{\mu}}{2} \max \left\{\left|h_{2}\right|,|J|\right\}+i \eta \operatorname{sign}\left(h_{1} J\right) \min \left\{\left|h_{1}\right|,|J|\right\}+i \xi \operatorname{sign}\left(h_{2} J\right) \min \left\{\left|h_{2}\right|,|J|\right\}\right]= \\
& =\frac{1}{c} \tilde{\chi}(i \eta, i \xi)+\frac{1}{c} \tilde{G}(i \eta+i \xi) \int_{\mathbb{R}} d h_{1} \int_{\mathbb{R}} d h_{2} \int_{\mathbb{R}} d y_{1} \int_{\mathbb{R}} d y_{2} e^{-i y_{1} h_{1}-i y_{2} h_{2}} e^{\tilde{G}\left(i y_{1}+i y_{2}\right)-c} \tilde{\chi}\left(i y_{1}, i y_{2}\right) \times \\
& \quad \times \int_{\mathbb{R}} d H p_{H}(H) e^{i\left(y_{1}+y_{2}\right) H} \exp \left[\frac{i \hat{\mu}}{2} \operatorname{sign}\left(h_{1}^{2}-h_{2}^{2}\right) \min \left\{\left|h_{1}+h_{2}\right|,\left|h_{1}-h_{2}\right|\right\}\right]- \\
& \quad-\int_{\mathbb{R}} d y_{1} \int_{\mathbb{R}} d y_{2} e^{\tilde{G}\left(i y_{1}+i y_{2}\right)-c} \tilde{\chi}\left(i y_{1}, i y_{2}\right) \int_{\mathbb{R}} d H p_{H}(H) e^{i\left(y_{1}+y_{2}\right) H} \int_{\mathbb{R}} d h_{1} \int_{\mathbb{R}} d h_{2} e^{-i y_{1} h_{1}-i y_{2} h_{2}} \times \\
& \quad \times \int_{\mathbb{R}} d J \rho(J) \exp \left[\frac{i \hat{\mu}}{2}\left[\max \left\{\left|h_{1}\right|,|J|\right\}-\max \left\{\left|h_{2}\right|,|J|\right\}\right]\right] \times \\
& \quad \times \exp \left[i \eta \operatorname{sign}\left(h_{1} J\right) \min \left\{\left|h_{1}\right|,|J|\right\}+i \xi \operatorname{sign}\left(h_{2} J\right) \min \left\{\left|h_{2}\right|,|J|\right\}\right],
\end{aligned}
$$

onde o limite $n \rightarrow 0$ foi tomado, além de continuações analíticas adequadas (Apêndice B.10). Agora, a tarefa de encontrar uma função $\varphi$ que satisfaça (3.44) foi convertida no problema de encontrar uma função de duas variáveis $\tilde{\chi}(i \eta, i \xi)$ que, a rigor, depende da soma das componentes $\hat{\mu}:=\sum_{\alpha=1}^{n} \mu_{\alpha}$ de um conjunto de vetores $\left\{\mu_{\alpha}\right\}$, introduzidos pelo teorema 3.1. São tratados os casos de autovalores longitudinais (Apêndice B.9) e transversais (Apêndice B.10), este último quando $\hat{\mu}=0$ e o caso mais geral, $\hat{\mu} \neq 0$. A resolução do problema de autovalores (3.46) é efetuada impondo um Ansatz,

$$
\tilde{\chi}_{\hat{\mu}}(i \eta, i \xi)=\sum_{\theta \in \mathbb{Z}} \sum_{u, v=0, \pm 1} K_{\theta}(u, v) e^{i u \eta J_{0}+i v \xi J_{0}+\frac{i \hat{a} \theta J_{0}}{2}}
$$

para os autovetores, onde $K_{\theta}(u, v) \in \mathbb{R}$ são coeficientes que não devem ser todos nulos - o que levaria a uma resposta trivial da equação secular. A adequação do Ansatz é comprovada a posteriori por autoconsistência, e os autovalores calculados são

$$
\lambda=\frac{1}{c}, \quad \frac{1}{c}-\frac{A}{c}, \quad \frac{1}{c}-\frac{A}{c}+\sqrt{a_{1} a_{-1}}
$$

e

$$
\lambda_{0}=\frac{1}{c}-\frac{A}{c}-\sqrt{a_{1} a_{-1}}
$$

onde este último é o menor dentre os encontrados. As equações (3.37), (3.38), (3.39) e (3.40) determinam $A, a_{1}$ e $a_{-1}$.

Trabalhos anteriores[55,56] sugerem a existência de uma fase vitrosa no estado fundamental. Esperase que numa fase paramagnética ou vidros de spin a distribuição do campo efetivo seja simétrica; em outros termos, $a_{k}=a_{-k}$, ou $B=C$. Por outro lado, numa fase ferromagnética haveria um desbalanceamento entre os $a_{k>0}$ e $a_{k<0}(B \neq C)$. Duas situações extremas serão examinadas, $m=0$ e $m \rightarrow \infty$; a primeira descreve um ferromagneto diluído sem campo externo, e uma fase ferromagnética é esperada (com instabilidade das fases paramagnética e vidros de spin).

As equações disponíveis para a caracterizar fase paramagnética são, de (3.37), (3.38), (3.40) e (3.49), 


$$
A=c e^{A-c} I_{m}(c-A)
$$

e

$$
\begin{aligned}
\lambda_{P} & =\frac{1}{c}-\frac{A}{c}-a_{1} \\
& =\frac{1}{c}-\frac{A}{c}-\frac{e^{A-c}}{2}\left[I_{m+1}(c-A)+I_{m-1}(c-A)\right],
\end{aligned}
$$

ao passo que a fase ferromagnética é governada pelas equações (3.38), (3.39) e (3.40), lembrando que $B \neq C$. A análise é feita sobre (3.49).

Os autovalores referentes às fases ferromagnética, $\lambda_{F}$, e paramagnética, $\lambda_{P}$, podem ser computados numericamente, como mostrado na tabela abaixo.

\begin{tabular}{|c|c|c|c|c|c|c|c|c|c|c|c|c|}
\hline $\mathrm{c}$ & $m$ & 0 & 1 & 2 & 3 & 4 & 5 & 6 & 7 & 8 & 9 & 10 \\
\hline \hline 3 & $\lambda_{P}$ & -0.180 & -0.066 & 0.103 & 0.225 & 0.292 & 0.320 & 0.330 & 0.333 & 0.333 & 0.333 & 0.333 \\
& $\lambda_{F}$ & 0.274 & 0.116 & - & - & - & - & - & - & - & - & - \\
\hline 4 & $\lambda_{P}$ & -0.188 & -0.116 & 0.013 & 0.121 & 0.191 & 0.227 & 0.242 & 0.248 & 0.249 & 0.250 & 0.250 \\
& $\lambda_{F}$ & 0.230 & 0.167 & - & - & - & - & - & - & - & -- & - \\
\hline 5 & $\lambda_{P}$ & -0.188 & -0.138 & -0.037 & 0.058 & 0.126 & 0.166 & 0.187 & 0.195 & 0.199 & 0.200 & 0.200 \\
& $\lambda_{F}$ & 0.193 & 0.167 & 0.082 & - & - & - & - & - & - & - & - \\
\hline 6 & $\lambda_{P}$ & -0.185 & -0.148 & -0.067 & 0.016 & 0.081 & 0.123 & 0.147 & 0.159 & 0.164 & 0.166 & 0.166 \\
& $\lambda_{F}$ & 0.164 & 0.153 & 0.115 & - & - & - & - & - & - & - & - \\
\hline 7 & $\lambda_{P}$ & -0.181 & -0.152 & -0.085 & -0.013 & 0.048 & 0.091 & 0.117 & 0.132 & 0.138 & 0.141 & 0.142 \\
& $\lambda_{F}$ & 0.142 & 0.137 & 0.120 & 0.072 & - & - & - & - & - & - & - \\
\hline 8 & $\lambda_{P}$ & -0.176 & -0.153 & -0.097 & -0.033 & 0.023 & 0.065 & 0.093 & 0.110 & 0.118 & 0.122 & 0.124 \\
& $\lambda_{F}$ & 0.125 & 0.123 & 0.115 & 0.092 & 0.024 & - & - & - & - & - & - \\
\hline 9 & $\lambda_{P}$ & -0.172 & -0.153 & -0.105 & -0.049 & 0.004 & 0.045 & 0.074 & 0.092 & 0.102 & 0.107 & 0.110 \\
& $\lambda_{F}$ & 0.111 & 0.110 & 0.106 & 0.095 & 0.068 & - & - & - & - & - & - \\
\hline 10 & $\lambda_{P}$ & -0.168 & -0.152 & -0.111 & -0.060 & -0.011 & 0.028 & 0.058 & 0.077 & 0.088 & 0.095 & 0.098 \\
& $\lambda_{F}$ & 0.100 & 0.100 & 0.098 & 0.092 & 0.079 & 0.044 & - & - & - & - & - \\
\hline
\end{tabular}

Tabela 3.1: Tabela $\lambda_{P}, \lambda_{F} \times m:=H_{R} / J_{0}$ para $c=3, \cdots, 10$.

Conforme esperado, a fase ferromagnética é estável para baixos campos aleatórios e a fase paramagnética para altos campos. Notar que, embora o autovalor correspondente à fase paramagnética exista sempre, a solução ferromagnética é possível apenas para $m:=H_{R} / J_{0}$ suficientemente pequeno. É possível provar (Apêndice B.12) que, além de ser negativo para $H_{R}=0$, o autovalor paramagnético tende a $1 / c$ no limite de campos infinitos; este último fato é observado na tabela acima, sendo o efeito mais pronunciado para conectividades menores. Notar que, a partir de $c=8$, existe uma região de $m:=H_{R} / J_{0}$ em que ambos autovalores são positivos, o que é um indício de transição de fase de primeira ordem em virtude da coexistência das duas fases, que os cálculos mostram ser estáveis.

$O$ que não se observa na tabela acima é uma região onde tanto $\lambda_{P}$ quanto $\lambda_{F}$ sejam negativos - o que caracterizaria uma fase vidros de spin. Os dados indicam que a quebra de simetria de permutação entre as réplicas é desnecessária no presente estudo (com campo aleatório múltiplo de $J_{0}$ ). Os dados para $c$ iguais a $8,16,32$ e 64 são mostrados nas figuras abaixo, evidenciando o alargamento da região de coexistência das duas fases com o aumento da conectividade; numericamente, isto ocorre para $c \geq 8$, indicando que abaixo deste limite a transição ordem-desordem seja de segunda ordem. 

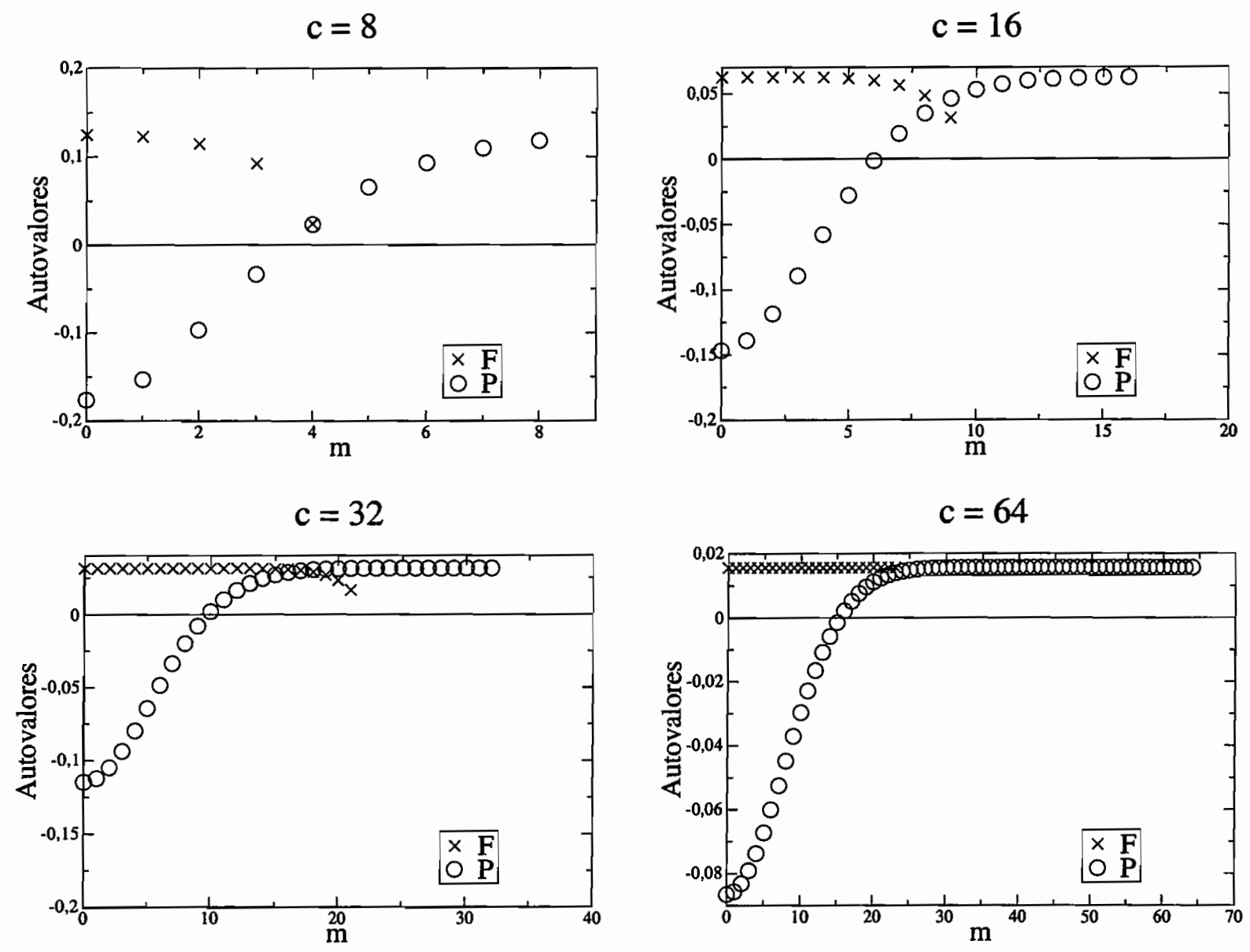

Figura 1: $\lambda_{0} \times m$ para $c=8,16,32$ e 64 .

\section{6- Considerações finais}

Contrariamente às expectativas de alguns trabalhos [55, 56], os resultados alcançados aqui não sugerem a presença de uma fase vidros de spin no estado fundamental. A solução réplica-simétrica mostrou-se estável para todo campo aleatório $H_{R}$ não-negativo, a fase ferromagnética sendo estável para baixos valores de $H_{R}$ e a fase paramagnética indicada para $H_{R}$ suficientemente grande. As soluções numéricas não forneceram um intervalo de campo aleatório (inteiro) em que os autovalores da matriz de estabilidade fossem ambos negativos.

Entretanto, as conclusões acima não devem ser consideradas definitivas. Recapitulando a análise, um ferromagneto diluído na presença de um campo externo aleatório bimodal foi tratado pelo truque de réplicas para a análise termodinâmica. A equação de estado (3.19), transformada em uma equação integral não linear (3.33), não pôde ser satisfatoriamente resolvida, e a análise prosseguiu com uma solução parcial, mas legítima, no estado fundamental e para campos $H_{R}$ múltiplos da integral de troca ferromagnética $J_{0}$, (3.34) (juntamente com (3.37), (3.38), (3.39) e (3.40)). Aqui, cabe a primeira observação que diz respeito à completeza das soluções encontradas. Espera-se que existam as soluções contínuas (tal como no caso vidros de spin sem campo externo [81]); ademais, a unicidade da solução obtida para campos discretos não foi provada. Novos autovalores provenientes de outras soluções poderiam estar escapando da análise, que então poderia levar a outras conclusões. 
Deve-se observar, no entanto, que esta não é a única fonte de incompleteza no estudo. A análise de estabilidade, traduzida na determinação dos autovalores de (3.46), foi realizada baseando-se no Ansatz (que foi verificada a posteriori) (3.47); novamente, a questão da (possível) unicidade desta solução não foi demonstrada. A possibilidade de existir outras soluções, que não foram exploradas, para a equação secular (3.46) permite conceber autovalores distintos dos obtidos e levar o exame da estabilidade das soluções réplica-simétricas a tomar outros rumos.

Os problemas de completeza supracitados não afligem a análise, por exemplo, do modelo de Sherrington e Kirkpatrick, e a explicação para tal diferença essencial entre este paradigma dos sistema desordenados e o modelo tratado aqui está relacionado com o desenvolvimento que se faz do truque de réplicas. Sabe-se que o limite $n \rightarrow 0$ deve ser tomado em algum estágio do estudo; no caso de SherringtonKirkpatrick, o número $n$, que é a dimensão do espaço de Hilbert $\mathfrak{H}_{S K}$, pode ser carregado até o fim da análise, e a completeza das soluções para a equação de estado ou os autovalores da matriz estabilidade pode ser averiguada. A continuação analítica $n \rightarrow 0$ neste caso, então, resume-se em um valor particular de um parâmetro que surge tanto nas soluções (da equação de estado) quanto nos autovalores obtidos. Por outro lado, no problema tratado nesta tese, o limite $n \rightarrow 0$ é tomado em uma etapa incipiente como recurso para possibilitar obter resultados heurísticos. Contrariamente ao caso de Sherrington e Kirkpatrick, onde esse limite pode ser tomado quando a noção de espaço de Hilbert deixa de ser relevante para a análise, no presente caso a continuação analítica é antecipada, quando então o espaço de Hilbert $\mathfrak{H}^{(n)}$ ainda é indispensável para o estudo. Daí, a transição de (3.18) para (3.30). Como conseqüência, ao preço de se obter soluções heurísticas, o conjunto $\mathfrak{H}^{(n)}$ é estendido para uma dimensão infinita. É natural, pois, que no presente caso estudos análogos como o de de Almeida e Thouless[71] sejam impossibilitados. 


\section{Apêndice A}

\section{A.1- Função de partição grã-canônica}

As informações termodinâmicas do modelo em estudo estão contidas na função de partição grãcanônica,

$$
\Xi_{\Lambda_{N}}(\beta, \mu)=\int_{\mathbb{R}^{\Lambda_{N}}} \exp \left[-\beta H\left(\left\{S_{x}\right\}\right)-\beta \mu \sum_{x \in \Lambda_{N}} S_{x}^{2}\right] \prod_{x \in \Lambda_{N}} d S_{x},
$$

sujeita ao vínculo esférico

$$
\left\langle\sum_{x \in \Lambda_{N}} S_{x}^{2}\right\rangle:=-\frac{1}{\beta} \frac{\partial}{\partial \mu} \ln \Xi_{\Lambda_{N}}(\beta, \mu)=N
$$

com a função de Hamilton dada por

$$
H\left(\left\{S_{x}\right\}\right)=-\frac{1}{2} \sum_{x, x^{\prime} \in \Lambda_{N}} J\left(x, x^{\prime}\right) S_{x} S_{x^{\prime}}-h \sum_{x \in \Lambda_{N}} S_{x}
$$

e a integral de troca por

$$
J\left(x, x^{\prime}\right)=J\left(x-x^{\prime}\right)=\left\{\begin{array}{lll}
R J & , x-x^{\prime}= \pm e_{i} & i \in\{1, \cdots, m\} \\
S J & , x-x^{\prime}= \pm 2 e_{i} & i \in\{1, \cdots, m\} \\
J \quad, \quad x-x^{\prime}= \pm e_{i} & i \in\{m+1, \cdots, d\} \\
0 & , x-x^{\prime} \neq \pm e_{i}, \pm 2 e_{j} & i \in\{1, \cdots, d\} \text { e } j \in\{1, \cdots, m\}
\end{array}\right.
$$

Os parâmetros acima foram definidos no capítulo 2. Notar, comparando com (2.8), a inclusão de um campo externo auxiliar $h$ na função de Hamilton. O passo inicial para a análise é escrever a função de partição grã-canônica no espaço recíproco mediante as transformadas de Fourier

$$
\hat{S}_{k}=\frac{1}{\sqrt{N}} \sum_{x \in \Lambda_{N}} S_{x} e^{-i k x} \quad \text { e } \quad \hat{J}(k)=\sum_{x \in \Lambda_{N}} J(x) e^{-i k x}
$$


É importante lembrar que as condições de contorno adotadas são periódicas. De (A.4), (A.5) e da realidade de $S_{x}$, é imediato que $\hat{J}(k)=\hat{J}(-k)$ e $\hat{S}_{-k}=\hat{S}_{k}^{*}$. Divide-se a primeira zona de Brillouin $\hat{\Lambda}_{N}=\left\{\frac{\pi x}{L+1}, x \in \Lambda_{N}\right\}$ em três subconjuntos disjuntos, $\{0\}, \hat{\Lambda}_{+}$e $\hat{\Lambda}_{-}$, tais que $\hat{\Lambda}_{N}=\{0\} \cup \hat{\Lambda}_{+} \cup \hat{\Lambda}_{-}$e se $k \in \hat{\Lambda}_{+}$, então $-k \in \hat{\Lambda}_{-}$. No espaço recíproco, tem-se

$$
\hat{J}(k)=2 J\left[R \sum_{i=1}^{m} \cos k_{i}+S \sum_{i=1}^{m} \cos \left(2 k_{i}\right)+\sum_{i=m+1}^{d} \cos k_{i}\right]
$$

e

$$
\begin{aligned}
\Xi_{\hat{\Lambda}_{N}}(\beta, \mu)= & \int_{\mathbb{R}^{N}} \exp \left\{-\beta \sum_{k \in \hat{\Lambda}_{+} \cup \hat{\Lambda}_{-}}\left[\mu-\frac{1}{2} \hat{J}(k)\right]\left|\hat{S}_{k}\right|^{2}-\beta\left[\mu-\frac{1}{2} \hat{J}(0)\right] \hat{S}_{0}^{2}+\beta h \sqrt{N} \hat{S}_{0}\right\} \times \\
& \times d \hat{S}_{0} \prod_{k \in \hat{\Lambda}_{+} \cup \hat{\Lambda}_{-}} d \hat{S}_{k} d \hat{S}_{k}^{*}
\end{aligned}
$$

A transformação ortogonal

$$
\left\{\begin{array} { l l } 
{ \hat { S } _ { 0 } = x _ { 0 } } & { ( k = 0 ) } \\
{ \hat { S } _ { k } = \frac { 1 } { \sqrt { 2 } } ( x _ { k } + i y _ { k } ) } & { ( k \in \hat { \Lambda } _ { + } ) }
\end{array} , \text { onde } \left\{\begin{array}{l}
x_{k}=x_{-k} \in \mathbb{R} \\
y_{k}=y_{-k} \in \mathbb{R}
\end{array},\right.\right.
$$

é útil para o prosseguimento do cálculo. A função de partição grã - canônica assume, então, a forma

$$
\begin{aligned}
\Xi_{\hat{\Lambda}_{N}}(\beta, \mu, h)= & \int_{\mathbb{R}} \exp \left\{-\beta\left[\mu-\frac{1}{2} \hat{J}(0)\right] x_{0}^{2}+\beta h \sqrt{N} x_{0}\right\} d x_{0} \times \\
& \times \int_{\mathbb{R}^{N-1}} \exp \left\{-\beta\left[\mu-\frac{1}{2} \hat{J}(k)\right]\left[\frac{\left.x_{k}^{2}+y_{k}^{2}\right]}{2}\right]\right\} \prod_{k \in \hat{\Lambda}_{+} \cup \hat{\Lambda}_{-}} d x_{k} d y_{k} \\
= & \left(\frac{\pi}{\beta}\right)^{\frac{N}{2}} \exp \left\{\frac{\beta h^{2} N}{4\left[\mu-\frac{1}{2} \hat{J}(0)\right]}\right\} \prod_{k \in \hat{\Lambda}_{N}}\left[\mu-\frac{1}{2} \hat{J}(k)\right]^{-\frac{1}{2}} .
\end{aligned}
$$

O cálculo acima é permitido para

$$
\mu>\mu_{c}:=\max _{k \in \hat{\Lambda}_{N}}\left\{\frac{1}{2} \hat{J}(k)\right\} .
$$

A energia livre pode ser escrita como

$$
\begin{aligned}
f_{\Lambda_{N}}(\beta, \mu, h) & =-\frac{1}{\beta N} \ln \Xi_{\hat{\Lambda}_{N}}(\beta, \mu, h) \\
& =-\frac{1}{2 \beta} \ln \left(\frac{\pi}{\beta}\right)-\frac{h^{2}}{4\left[\mu-\frac{1}{2} \hat{J}(0)\right]}-\frac{1}{2 \beta N} \sum_{k \in \hat{\Lambda}_{N}} \ln \left[\mu-\frac{1}{2} \hat{J}(k)\right] .
\end{aligned}
$$

Tomando $h=0$ em (A.9), recupera-se (2.10). 


\section{A.2- Dimensões críticas}

Esta seção é dedicada à determinação da dimensão crítica $d_{c}$. Para $d \leq d_{c}$ a integral

$$
Y(d):=\int_{\hat{\Lambda}} \frac{d^{d} k}{(2 \pi)^{d}} \frac{1}{\hat{J}\left(k_{c}\right)-\hat{J}(k)} \quad\left(=\beta_{c}\right)
$$

diverge. A primeira zona de Brillouin $\hat{\Lambda}=[-\pi, \pi]^{d}$ é limitada e a integral não apresenta problemas no ultravioleta. A convergência ou a divergência está ligada ao domínio que contém o vetor de onda $k_{c}$. Na região próxima deste ponto,

$$
\begin{aligned}
\hat{J}\left(k_{c}\right)-\hat{J}(k)= & J\left[c_{2} \sum_{i=1}^{m}\left(k_{i}-q_{c}\right)^{2}+\sum_{i=m+1}^{d} k_{i}^{2}\right]-\frac{J}{3} c_{3} \sum_{i=1}^{m}\left(k_{i}-q_{c}\right)^{3}+ \\
& +\frac{J}{12}\left[c_{4} \sum_{i=1}^{m}\left(k_{i}-q_{c}\right)^{4}+\sum_{i=m+1}^{d} k_{i}^{4}\right]+\cdots
\end{aligned}
$$

onde

$$
\begin{gathered}
c_{2}=R \cos \left(q_{c}\right)+4 S \cos \left(2 q_{c}\right), \\
c_{3}=R \sin \left(q_{c}\right)+8 S \sin \left(2 q_{c}\right), \\
c_{4}=-R \cos \left(q_{c}\right)-16 S \cos \left(2 q_{c}\right), \\
k_{c}=(\underbrace{q_{c}, \cdots, q_{c}}_{m}, \underbrace{0, \cdots, 0}_{d-m}),
\end{gathered}
$$

e

$$
q_{c}= \begin{cases}0, & R>0 \text { e } S>-\frac{R}{4}, \\ \pi, & R<0 \text { e } S>-\frac{R}{4}, . \\ \pm \phi, & S<-\frac{|R|}{4},\end{cases}
$$

onde $\phi:=\arccos \left(-\frac{R}{4 S}\right)$.

Nota-se que $c_{2} \geq 0$. A situação $c_{2}=0$ realiza-se somente nas linhas $R+4 S=0 \quad\left(q_{c}=0\right)$ e $R-4 S=0\left(q_{c}=\pi\right)$, quando também $c_{3}=0$ e os termos de quarta ordem desempenham um papel importante na expansão (A.13) e no comportamento da integral de $Y(d)$.

O estudo será feito nas seguintes etapas: 


\begin{tabular}{|c|c|c|}
\hline \multirow{2}{*}{$m \neq 0, d-m \neq 0$} & $c_{2}>0$ & Caso 1 \\
\cline { 2 - 3 } & $c_{2}=0$ & Caso 2 \\
\hline \multirow{2}{*}{$m \neq 0, d-m=0$} & $c_{2}>0$ & Caso 3 \\
\cline { 2 - 3 } & $c_{2}=0$ & Caso 4 \\
\hline$m=0, d-m \neq 0$ & & Caso 5 \\
\hline
\end{tabular}

Antes de prosseguir, para $a, b \in \mathbb{N}$ (com $a \leq b$ ), $x \in \mathbb{R}$ e $\delta \in \mathbb{R}_{+}$, definem-se os conjuntos $U_{a}^{b}(x, \delta$ ), $K_{a}^{b}(x, \delta)$ e $B_{\delta}\left(x_{0}, y_{0}\right)$,

$$
\begin{aligned}
& U_{a}^{b}(x, \delta):=\left\{k \in \hat{\Lambda}: \sum_{i=a}^{b}\left(k_{i}-x\right)^{2}<\delta^{2}\right\}, \\
& K_{a}^{b}(x, \delta):=\left\{k \in \hat{\Lambda}: \max _{i=a, \cdots, b}\left|k_{i}-x\right|<\delta\right\}
\end{aligned}
$$

e

$$
B_{\delta}\left(x_{0}, y_{0}\right):=\left\{(x, y) \in \mathbb{R}^{2}:\left(x-x_{0}\right)^{2}+\left(y-y_{0}\right)^{2}<\delta^{2}\right\}
$$

que serão úteis na análise que se segue. $\mathrm{O}$ conjunto $U_{a}^{b}(x, \delta)\left(K_{a}^{b}(x, \delta)\right)$ é um subconjunto da primeira zona de Brillouin cuja projeção nas direções $a$ a $b$ é uma hiperesfera (hipercubo) de raio (lado) $\delta(2 \delta)$ centrada(o) em $(\underbrace{0, \cdots, 0}_{a-1}, \underbrace{x, \cdots, x}_{b-a+1}, \underbrace{0, \cdots, 0}_{d-b})$. O conjunto $B_{\delta}\left(c_{1}, c_{2}\right)$ é uma bola aberta em $\mathbb{R}^{2}$ centrada em $\left(c_{1}, c_{2}\right)$. Por fim, é necessário introduzir o fator de contagem $d_{f c}\left(q_{c}, m\right)$, dado por

$$
d_{f c}\left(q_{c}, m\right):=\left\{\begin{array}{ccc}
1 & , & q_{c}=0, \pi \\
2^{m} & , & q_{c} \neq 0, \pi
\end{array} .\right.
$$

Este fator distingue a situação em que $q_{c}= \pm \phi$ da outra onde $q_{c}=0$ ou $q_{c}=\pi$. O intervalo $[-\pi, \pi]$ (mais precisamente, $(-\pi, \pi])$ compreende apenas um valor para 0 e $\pi$; por outro lado, $\cos \phi=-\frac{R}{4 S}$ é satisfeito não apenas para $\phi$, mas também para $-\phi$, e esta degenerescência impõe um fator dois nos resultados abaixo para cada uma das $m$ direções de competição onde $q_{c}=\phi$. O procedimento adotado será desconsiderar as contribuições de $-\phi$ e corrigir o resultado final impondo o fator $d_{f c}\left(q_{c}, m\right)$.

$\diamond \operatorname{Casos} 1$ e $2(m \neq 0, d-m \neq 0)$

Estes casos tratam de um modelo em que existe ao menos uma direção onde está presente uma interação de segundos vizinhos e pelo menos uma direção com apenas interações de primeiros vizinhos. A dimensão mínima que é exigida para esta situação é, pois, 2 .

* Caso $1\left(c_{2}>0\right)$

Neste caso, os termos de ordem superior ou igual a três em (A.13) podem ser desprezados frente aos termos de segunda ordem para uma região de integração $U_{1}^{m}\left(q_{c}, \delta_{1}\right) \cap U_{m+1}^{d}\left(0, \delta_{2}\right)$ suficientemente 
próxima do ponto crítico $k_{c}$, bastando examinar a situação de baixos valores de $\delta_{1}$ e $\delta_{2}$. Escreve-se a função $Y(d)$ como

$$
\begin{aligned}
Y(d)= & \frac{2}{J} d_{f c}\left(q_{c}, m\right) \underset{U_{1}^{m}\left(q_{c}, \delta_{1}\right) \cap U_{m+1}^{d}\left(0, \delta_{2}\right)}{\frac{d^{d} k}{(2 \pi)^{d}}} \frac{1}{c_{2} \sum_{i=1}^{m}\left(k_{i}-q_{c}\right)^{2}+\mathcal{O}\left(\delta_{1}^{3}\right)+\sum_{i=m+1}^{d} k_{i}^{2}+\mathcal{O}\left(\delta_{2}^{4}\right)}+ \\
& +\underset{\left.\hat{\Lambda} \backslash\left(U_{1}^{m}\left(q_{c}, \delta_{1}\right) \cap U_{m+1}^{d}\left(0, \delta_{2}\right)\right) \cup\left(U_{1}^{m}\left(-q_{c}, \delta_{1}\right) \cap U_{m+1}^{d}\left(0, \delta_{2}\right)\right)\right]}{\frac{d^{d} k}{(2 \pi)^{d}} \frac{1}{\hat{J}\left(k_{c}\right)-\hat{J}(k)} .}
\end{aligned}
$$

Reunir $\mathcal{O}\left(\delta_{1}^{3}\right)$ e $\mathcal{O}\left(\delta_{2}^{4}\right)$ em um único termo, através da escolha $\delta_{1}=\delta_{2}^{4 / 3}$, é um procedimento simplificador e não compromete a análise, visto que a convergência da integral em $Y(d)$ independe do valor de $\delta_{1}$ ou $\delta_{2}$. Estes dois têm o papel meramente auxiliar de delimitar, convenientemente, a região que engloba o ponto de interesse $k_{c}$ para então calcular a integral. Para $\delta_{2}{ }^{11}$ suficientemente pequeno, tem-se

$$
\begin{aligned}
Y(d)= & \frac{2}{J} d_{f c}\left(q_{c}, m\right) \underbrace{\int_{U_{1}^{m}\left(0, \delta_{1}\right) \cap U_{m+1}^{d}\left(0, \delta_{2}\right)} \frac{d^{d} k}{(2 \pi)^{d}} \frac{1}{c_{2} \sum_{i=1}^{m} k_{i}^{2}+\sum_{i=m+1}^{d} k_{i}^{2}}}_{Y_{1}(d)}+\mathcal{O}\left(\delta_{2}^{2}\right)+ \\
& +\int_{\hat{\Lambda} \backslash\left[\left(U_{1}^{m}\left(q_{c}, \delta_{1}\right) \cap U_{m+1}^{d}\left(0, \delta_{2}\right)\right) \cup\left(U_{1}^{m}\left(-q_{c}, \delta_{1}\right) \cap U_{m+1}^{d}\left(0, \delta_{2}\right)\right)\right]} \frac{d^{d} k}{(2 \pi)^{d}} \frac{1}{\hat{J}\left(k_{c}\right)-\hat{J}(k)}
\end{aligned}
$$

$\operatorname{Em} \hat{\Lambda} \backslash\left[\left(U_{1}^{m}\left(q_{c}, \delta_{1}\right) \cap U_{m+1}^{d}\left(0, \delta_{2}\right)\right) \cup\left(U_{1}^{m}\left(-q_{c}, \delta_{1}\right) \cap U_{m+1}^{d}\left(0, \delta_{2}\right)\right)\right]$, a função $\left[\hat{J}\left(k_{c}\right)-\hat{J}(k)\right]^{-1}$ é limitada; por conseguinte, o último termo de (A.22) é limitado. A finitude da função $Y$ está condicionada, pois, ao comportamento da integral $Y_{1}(d)$. A análise prossegue tratando esta integral em coordenadas (hiper)esféricas,

$$
\begin{aligned}
Y_{1}(d) & =\frac{1}{(2 \pi)^{d}} \int_{0}^{\delta_{1}} d u u^{m-1} \int_{0}^{\delta_{2}} d k \frac{k^{d-m-1}}{c_{2} u^{2}+k^{2}} \int d \Omega_{u} d \Omega_{k} \\
& =\frac{1}{(2 \pi)^{d}} c_{2}^{\frac{d-m-2}{2}} \underbrace{\int_{0}^{\delta_{1}} d u u^{m-1} \int_{0}^{\frac{\delta_{2}}{\sqrt[c^{2}]{2}} d y \frac{y^{d-m-1}}{u^{2}+y^{2}}} \int d \Omega_{u} d \Omega_{k}}_{Y_{11}(d)}
\end{aligned}
$$

para $\left(u, \Omega_{u}\right) \subset U_{1}^{m}\left(0, \delta_{1}\right)$ e $\left(k, \Omega_{k}\right) \subset U_{m+1}^{d}\left(0, \delta_{2}\right)$, sendo $\Omega_{u}$ e $\Omega_{k}$ a parte angular do subespaço de $\hat{\Lambda}$ compreendido pelas dimensões 1 a $m$, e $m+1$ a $d$, respectivamente. Tomando uma bola aberta de raio $\eta$, definida em (A.19), e considerando $\eta>0$ suficientemente pequeno, tem-se $B_{\eta}\left(\frac{\delta_{1}}{2}, \frac{\delta_{2}}{2 \sqrt{c_{2}}}\right) \subset$ $\left[0, \delta_{1}\right] \times\left[0, \frac{\delta_{2}}{\sqrt{c_{2}}}\right]$ e, desta forma,

\footnotetext{
${ }^{11}$ Sabe-se que $\delta_{2}$ é função de $\delta_{1}$; esta dependência, porém, não será explicitada para não sobrecarregar a notação.
} 


$$
\begin{aligned}
Y_{11}(d) & =\int_{0}^{\delta_{1}} d u u^{m-1} \int_{0}^{\frac{\delta_{2}}{\sqrt{c_{2}}} d y \frac{y^{d-m-1}}{u^{2}+y^{2}}} \\
& =\int_{B_{\eta}\left(\frac{\delta_{1}}{2}, \frac{\delta_{2}}{2 \sqrt{c_{2}}}\right)} d u d y \frac{u^{m-1} y^{d-m-1}}{u^{2}+y^{2}}+\int_{\left(\left[0, \delta_{1}\right] \times\left[0, \frac{\delta_{2}}{\sqrt{c_{2}}}\right]\right) \backslash B_{\eta}\left(\frac{\delta_{1}}{2}, \frac{\delta_{2}}{2 \sqrt{c_{2}}}\right)} d u d y \frac{u^{m-1} y^{d-m-1}}{u^{2}+y^{2}} \\
& =\int_{0}^{2 \pi} d \theta \cos ^{m-1} \theta \sin ^{d-m-1} \theta \int_{0}^{\eta} d r r^{d-3}+\int_{\left(\left[0, \delta_{1}\right] \times\left[0, \frac{\delta_{2}}{\sqrt{c_{2}}}\right]\right) \backslash B_{\eta}\left(\frac{\delta_{1}}{2}, \frac{\delta_{2}}{2 \sqrt{c_{2}}}\right)} d u d y \frac{u^{m-1} y^{d-m-1}}{u^{2}+y^{2}}
\end{aligned}
$$

onde o último termo na equação acima é limitado e o penúltimo termo determina a convergência (ou não) de $Y_{11}(d)$, que converge para $d>2$, estabelecendo a dimensão crítica inferior como sendo 2 . Conclui-se que para $m \neq 0, d-m \neq 0$ e $c_{2}>0$, a integral em $Y(d)$ converge se, e somente se, $m>1$ ou $d-m>1$.

\section{* Caso $2\left(c_{2}=0\right)$}

A condição $c_{2}=0$ realiza-se necessariamente quando $q_{c}=0$ ou $\pi$; nesta situação, pode-se mostrar facilmente que $c_{3}=0$ (através da equação em (A.14)) e $d_{f c}\left(q_{c}, m\right)=1$. No caso 2, os termos quárticos da expansão (A.13) nas direções 1 a $m$ não podem ser desprezados. Utilizando um domínio hipercúbico em torno de $k_{c}$ (recorrendo a (A.18)), e procedendo analogamente ao caso 1, a função $Y(d)$, aqui, assume a forma

$$
\begin{aligned}
& Y(d)=\frac{2}{J} \int_{K_{1}^{m}\left(q_{c}, \delta_{1}\right) \cap K_{m+1}^{d}\left(0, \delta_{2}\right)} \frac{d^{d} k}{(2 \pi)^{d}} \frac{1}{\sum_{i=m+1}^{d} k_{i}^{2}+\mathcal{O}\left(\delta_{2}^{4}\right)+\frac{c_{4}}{12} \sum_{j=1}^{m}\left(k_{j}-q_{c}\right)^{4}+\mathcal{O}\left(\delta_{1}^{6}\right)}+ \\
& +\int_{\hat{\Lambda} \backslash\left(K_{1}^{m}\left(q_{c}, \delta_{1}\right) \cap K_{m+1}^{d}\left(0, \delta_{2}\right)\right)} \frac{d^{d} k}{(2 \pi)^{d}} \frac{1}{\hat{J}\left(k_{c}\right)-\hat{J}(k)} \\
& =\frac{2}{J} \underbrace{\underbrace{}_{K_{1}^{m}\left(0, \delta_{1}\right) \cap K_{m+1}^{d}\left(0, \delta_{2}\right)} \frac{d^{d} k}{(2 \pi)^{d}} \frac{1}{\sum_{i=m+1}^{d} k_{i}^{2}+\frac{c_{4}}{12} \sum_{j=1}^{m} k_{j}^{4}}}_{Y_{2}(d)}+\mathcal{O}\left(\delta_{1}^{2}\right)+ \\
& +\int_{\hat{\Lambda} \backslash\left(K_{\mathbf{1}}^{m}\left(q_{c}, \delta_{1}\right) \cap K_{m+1}^{d}\left(0, \delta_{2}\right)\right)} \frac{d^{d} k}{(2 \pi)^{d}} \frac{1}{\hat{J}\left(k_{c}\right)-\hat{J}(k)}
\end{aligned}
$$

onde, na última passagem, foi feita a escolha $\delta_{2}=\delta_{1}^{\frac{3}{2}}$. O último termo da equação acima é limitado, e a finitude de $Y(d)$ depende somente de 


$$
\begin{aligned}
Y_{2}(d) & =\int_{\left[-\delta_{1}, \delta_{1}\right]^{m} \times\left[-\delta_{2}, \delta_{2}\right]^{d-m}} \frac{d^{d} k}{(2 \pi)^{d}} \frac{1}{\sum_{i=m+1}^{d} k_{i}^{2}+\frac{c_{4}}{12} \sum_{j=1}^{m} k_{j}^{4}} \\
& =\frac{1}{(2 \pi)^{d}} \int_{\left[-\delta_{2}, \delta_{2}\right]^{d-m}} d k_{m+1} \cdots d k_{d} 2^{m} \int_{\left[0, \delta_{1}\right]^{m}} \frac{d k_{1} \cdots d k_{m}}{\sum_{i=m+1}^{d} k_{i}^{2}+\frac{c_{4}}{12} \sum_{j=1}^{m} k_{j}^{4}} \\
& =\frac{1}{(2 \pi)^{d}} \int_{\left[-\delta_{2}, \delta_{2}\right]^{d-m}} d k_{m+1} \cdots d k_{d} \frac{2^{m}}{2^{m}}\left(\frac{12}{c_{4}}\right)^{\frac{m}{4}} \int_{\left[0, \delta_{1}\right]^{m}} \frac{d u_{1} \cdots d u_{m}}{\sqrt{\prod_{j=1}^{m} u_{j}} \frac{1}{\sum_{i=m+1}^{d} k_{i}^{2}+\sum_{l=1}^{m} u_{l}^{2}}} \\
& =\frac{1}{(2 \pi)^{d}}\left(\frac{192}{c_{4}}\right)^{\frac{m}{4}} \int_{\left[-\delta_{1}, \delta_{1}\right]^{m}} \underbrace{}_{d^{\left.d-\delta_{2}, \delta_{2}\right]^{d-m}} \int_{Y_{21}(d)} \frac{d^{m} u}{\sqrt{\prod_{j=1}^{m}\left|u_{j}\right|} \frac{1}{\sum_{i=m+1}^{d} k_{i}^{2}+\sum_{l=1}^{m} u_{l}^{2}}}}
\end{aligned}
$$

Definindo, para $\eta \in \mathbb{R}_{+}$suficientemente pequeno, os conjuntos $U_{1}^{m}(0, \eta) \subset\left[-\delta_{1}, \delta\right]^{m}$ e $U_{m+1}^{d}(0, \eta) \subset$ $\left[-\delta_{2}, \delta_{2}\right]^{d-m}$, vem

$$
\begin{aligned}
& Y_{21}(d)=\underbrace{\int_{U_{1}^{m}(0, \eta) \cap U_{m+1}^{d}(0, \eta)} \frac{d^{d} u}{\sqrt{\prod_{j=1}^{m}\left|u_{j}\right|}} \frac{1}{\sum_{i=m+1}^{d} u_{i}^{2}+\sum_{l=1}^{m} u_{l}^{2}}}_{Y_{211}(d)}+ \\
& +\int_{\left[-\delta_{1}, \delta_{1}\right] \times\left[-\delta_{2}, \delta_{2}\right] \backslash\left(U_{1}^{m}(0, \eta) \cap U_{m+1}^{d}(0, \eta)\right)} \frac{d^{d} u}{\sqrt{\prod_{j=1}^{m}\left|u_{j}\right|}} \frac{1}{\sum_{i=m+1}^{d} u_{i}^{2}+\sum_{l=1}^{m} u_{l}^{2}} .
\end{aligned}
$$

Pela limitação do último termo em (A.27), é suficiente verificar a convergência de $Y_{211}(d)$, que pode ser expresso em coordenadas (hiper)esféricas $\left(r,\left\{\phi_{i}\right\}\right)$, onde $r$ é o raio e $\left\{\phi_{i}\right\}$ representa a parte angular. É imediato que

$$
\begin{aligned}
Y_{211}(d)= & \int \frac{d \Omega_{r}}{\sqrt{\prod_{i=1}^{m}\left|\phi_{i}\right|}} \int d \Omega_{s} \int_{0}^{\eta} \frac{d r r^{m-1}}{r^{\frac{m}{2}}} \int_{0}^{\eta} \frac{d s s^{d-m-1}}{r^{2}+s^{2}} \\
= & \int \frac{d \Omega_{r}}{\sqrt{\prod_{i=1}^{m}\left|\phi_{i}\right|}} \int d \Omega_{s}\left(\int_{[0, \eta] \times[0, \eta] \backslash B_{\gamma}\left(\frac{\eta}{2}, \frac{\eta}{2}\right)} \frac{d r d s r^{\frac{m}{2}-1} s^{d-m-1}}{r^{2}+s^{2}}\right. \\
& \left.+\int_{0}^{2 \pi} d \theta \cos ^{\frac{m}{2}-1} \theta \sin ^{d-m-1} \theta \int_{0}^{\gamma} d \rho \rho^{d-\frac{m}{2}-3}\right) .
\end{aligned}
$$

A convergência da integral depende unicamente do último termo, que a assegura para $d-\frac{m}{2}>2$. Estas observações são possíveis em virtude do seguinte lema:

Lema A1: Em uma hiperesfera de dimensão $d \geq 2$, a integral em todo o ângulo sólido

$$
\int \frac{d \Omega}{\sqrt{\prod_{i=1}^{d}\left|\phi_{i}\right|}}
$$


em que $\left\{\phi_{i}\right\}$ é o conjunto das componentes angulares, é limitada (por $\frac{\sqrt{2}}{\pi}(2 \pi)^{d}$ ).

A demonstração encontra-se no Apêndice A.3. Este lema mostra que a parte angular da integral em $Y_{211}(d)$ é finita e não nula, e as eventuais divergências (em função da dimensão) são inteiramente apontadas pela integral da parte radial; em outras palavras, a condição de finitude de $Y_{2}(d)$ é sempre $d-\frac{m}{2}>2$. A fim de explicitar a dimensão crítica, define-se $r:=m / d$, que éum número fixo, e a condição de finitude de $Y_{2}(d)$ é $2 d-r d>4$, o que leva à dimensão crítica $d_{c}=\frac{4}{2-r}$.

$\diamond \operatorname{Casos} 3$ e $4(m \neq 0, d-m=0)$

Quando $m=d$, a direção de competição está presente isotropicamente em todos os eixos. Esta situação particular ilustra o modelo esférico com interações isotrópicas de primeiros e segundos vizinhos. $\mathrm{O}$ análogo de (A.21) nesta situação é

$$
\begin{aligned}
Y_{3,4}(d)= & \frac{2}{J} \int_{U_{1}^{d}\left(q_{c}, \delta\right)} \frac{d^{d} k}{(2 \pi)^{d}} \frac{d_{f c}\left(q_{c}, m\right)}{c_{2} \sum_{i=1}^{d}\left(k_{i}-q_{c}\right)^{2}-\frac{c_{3}}{3} \sum_{i=1}^{d}\left(k_{i}-q_{c}\right)^{3}+\frac{c_{4}}{12} \sum_{i=1}^{d}\left(k_{i}-q_{c}\right)^{4}+\mathcal{O}\left(\delta^{5}\right)}+ \\
& +\int_{\hat{\Lambda} \backslash\left(U_{1}^{d}\left(q_{c}, \delta\right) \cup U_{1}^{d}\left(-q_{c}, \delta\right)\right)} \frac{d^{d} k}{(2 \pi)^{d}} \frac{1}{\hat{J}\left(k_{c}\right)-\hat{J}(k)}
\end{aligned}
$$

O último termo na equação acima é limitado e não participa na análise da finitude de $Y_{3,4}(d)$

* Caso $3\left(c_{2}>0\right)$

Nesta situação, os termos superiores a $\mathcal{O}\left(\delta^{3}\right)$ podem ser desprezados diante do termo quadrático, chegando-se a

$$
\begin{aligned}
Y_{3}(d) & =\frac{2}{J} d_{f c}\left(q_{c}, d\right) \int_{U_{1}^{d}\left(q_{c}, \delta\right)} \frac{d^{d} k}{(2 \pi)^{d}} \frac{1}{c_{2} \sum_{i=1}^{d}\left(k_{i}-q_{c}\right)^{2}+\mathcal{O}\left(\delta^{3}\right)}+\int_{\hat{\Lambda} \backslash\left(U_{1}^{d}\left(q_{c}, \delta\right) \cup U_{1}^{d}\left(-q_{c}, \delta\right)\right)} \frac{d^{d} k}{(2 \pi)^{d}} \frac{1}{\hat{J}\left(k_{c}\right)-\hat{J}(k)} \\
& =\frac{2}{J} d_{f c}\left(q_{c}, d\right) \int_{\int_{U_{3}^{d}(d)} \frac{d^{d} k}{\left(2 \pi q_{c}, \delta\right)} \frac{1}{c_{2} \sum_{i=1}^{d}\left(k_{i}-q_{c}\right)^{2}}}+\mathcal{O}(\delta)+\int_{\hat{\Lambda} \backslash\left(U_{1}^{d}\left(q_{c}, \delta\right) \cup U_{1}^{d}\left(-q_{c}, \delta\right)\right)} \frac{d^{d} k}{(2 \pi)^{d}} \frac{1}{\hat{J}\left(k_{c}\right)-\hat{J}(k)} .
\end{aligned}
$$

Por procedimento análogo ao caso 1, escreve-se $Y_{3}(d)$ em coordenadas (hiper)esféricas, obtendo

$$
Y_{3}(d)=\frac{2 \pi^{\frac{d}{2}}}{\Gamma\left(\frac{d}{2}\right)} \int_{0}^{\delta} \frac{d k k^{d-1}}{(2 \pi)^{d}} \frac{1}{c_{2} k^{2}},
$$

donde se nota que a dimensão crítica neste caso é 2 .

* Caso $4\left(c_{2}=0\right)$ 
Com a ausência dos termos quadráticos e cúbicos (a situação $c_{2}=0$ realiza-se somente quando $q_{c}=0$ ou $q_{c}=\pi$, quando então $c_{3}=0$ e $\left.d_{f c}\left(q_{c}, d\right)=1\right)$,

$$
\begin{aligned}
Y(d) & =\frac{24}{J} \int_{K_{1}^{d}\left(q_{c}, \delta\right)} \frac{d^{d} k}{(2 \pi)^{d}} \frac{1}{c_{4} \sum_{i=1}^{d}\left(k_{i}-q_{c}\right)^{4}+\mathcal{O}\left(\delta^{6}\right)}+\int_{\hat{\Lambda} \backslash K_{1}^{d}\left(q_{c}, \delta\right)} \frac{d^{d} k}{(2 \pi)^{d}} \frac{1}{\hat{J}\left(k_{c}\right)-\hat{J}(k)} \\
& =\frac{24}{J} \int_{K_{K_{1}^{d}\left(q_{c}, \delta\right)} \frac{d^{d} k}{(2 \pi)^{d}} \frac{1}{c_{4} \sum_{i=1}^{d}\left(k_{i}-q_{c}\right)^{4}}}+\mathcal{O}\left(\delta^{2}\right)+\int_{\hat{\Lambda} \backslash K_{1}^{d}\left(q_{c}, \delta\right)} \frac{d^{d} k}{(2 \pi)^{d}} \frac{1}{\hat{J}\left(k_{c}\right)-\hat{J}(k)} .
\end{aligned}
$$

A finitude de $Y(d)$ depende somente de $Y_{4}(d)$, visto que o último termo é limitado. Então

$$
\begin{aligned}
Y_{4}(d) & =\int_{K_{1}^{d}\left(q_{c}, \delta\right)} \frac{d^{d} k}{(2 \pi)^{2}} \frac{1}{c_{4} \sum_{i=1}^{d}\left(k_{i}-q_{c}\right)^{4}}=\int_{K_{1}^{d}(0, \delta)} \frac{d^{d} k}{(2 \pi)^{2}} \frac{1}{c_{4} \sum_{i=1}^{d} k_{i}^{4}} \\
& =\int_{\left[0, \delta^{2}\right]^{d}} \frac{d^{d} u}{(2 \pi)^{d}} \frac{1}{c_{4} \sqrt{\prod_{i=1}^{d} u_{i} \sum_{j=1}^{d} u_{j}^{2}}}=\frac{1}{2^{d}} \int_{\left[-\delta^{2}, \delta^{2}\right]^{d}} \frac{d^{d} u}{(2 \pi)^{d}} \frac{1}{c_{4} \sqrt{\left|\prod_{i=1}^{d} u_{i}\right| \sum_{j=1}^{d} u_{j}^{2}}} .
\end{aligned}
$$

Para $\eta \in \mathbb{R}_{+}$suficientemente pequeno, existe um $U_{1}^{d}(0, \eta) \subset\left[-\delta^{2}, \delta^{2}\right]^{d}$. Então,

$$
Y_{4}(d)=\frac{1}{2^{d}} \underbrace{\int_{U_{1}^{d}(0, \eta)} \frac{d^{d} u}{(2 \pi)^{d}} \frac{1}{c_{4} \sqrt{\left|\prod_{i=1}^{d} u_{i}\right|} \sum_{j=1}^{d} u_{j}^{2}}}_{Y_{41}(d)}+\frac{1}{2^{d}} \int_{\left[-\delta^{2}, \delta^{2}\right]^{d} \backslash U_{1}^{d}(0, \delta)} \frac{d^{d} u}{(2 \pi)^{d}} \frac{1}{c_{4} \sqrt{\left|\prod_{i=1}^{d} u_{i}\right| \sum_{j=1}^{d} u_{j}^{2}}},
$$

onde o segundo termo no segundo membro é limitado. Em coordenadas (hiper)esféricas,

$$
u_{i}=r \phi_{i}\left(\Omega_{u}\right) \quad \text { com } \quad \sum_{i=1}^{d} u_{i}^{2}=r^{2},
$$

onde $\Omega_{u}$ representa a parte angular de $u$, chega-se a

$$
Y_{41}(d)=\int \frac{d \Omega}{c_{4} \sqrt{\left|\prod_{i=i}^{d} \phi_{i}(\Omega)\right|}} \int_{0}^{\eta} \frac{d u u^{d-1}}{\sqrt{u^{d}} u^{2}}
$$

e, pelo lema A.1, a dimensão crítica é 4 .

$\diamond$ Caso $5(m=0, d-m \neq 0)$ 
Este último caso retrata um modelo onde não há interações de segundos vizinhos, reduzindo-se a um ferromagneto simples. A integral em $Y(d)$ é escrita como

$$
\begin{aligned}
Y(d) & =\frac{2}{J} \int_{U_{1}^{d}(0, \delta)} \frac{d^{d} k}{(2 \pi)^{d}} \frac{1}{\sum_{i=1}^{d} k_{i}^{2}+\mathcal{O}\left(\delta^{3}\right)}+\int_{\hat{\Lambda} \backslash U_{1}^{d}(0, \delta)} \frac{d^{d} k}{(2 \pi)^{d}} \frac{1}{\hat{J}\left(k_{c}\right)-\hat{J}(k)} \\
& =\frac{2}{J} \underbrace{\int_{U_{1}^{d}(0, \delta)} \frac{d^{d} k}{(2 \pi)^{d}} \frac{1}{\sum_{i=1}^{d} k_{i}^{2}}}_{Y_{5}(d)}+\mathcal{O}(\delta)+\int_{\hat{\Lambda} \backslash U_{1}^{d}(0, \delta)} \frac{d^{d} k}{(2 \pi)^{d}} \frac{1}{\hat{J}\left(k_{c}\right)-\hat{J}(k)} .
\end{aligned}
$$

Escrevendo $Y_{5}(d)$ em coordenadas (hiper)esféricas,

$$
Y_{5}(d)=\frac{2 \pi^{\frac{d}{2}}}{\Gamma\left(\frac{d}{2}\right)} \int_{0}^{\delta} \frac{d k k^{d-1}}{(2 \pi)^{d}} \frac{1}{k^{2}},
$$

obtém-se a dimensão crítica, que é 2 .

Reunindo os resultados obtidos, tem-se, lembrando que $r:=m / d$,

\begin{tabular}{|c|l|l|l|}
\hline \multirow{2}{*}{$m \neq 0, d-m \neq 0$} & Caso 1 & $|R|+4 S \neq 0$ & $d_{c}=2$ \\
\cline { 2 - 4 } & Caso 2 & $|R|+4 S=0$ & $d_{c}=\frac{4}{2-r}$ \\
\hline \multirow{2}{*}{$m \neq 0, d-m=0$} & Caso 3 & $|R|+4 S \neq 0$ & $d_{c}=2$ \\
\cline { 2 - 4 } & Caso 4 & $|R|+4 S=0$ & $d_{c}=4$ \\
\hline$m=0, d-m \neq 0$ & Caso 5 & & $d_{c}=2$ \\
\hline
\end{tabular}

A dimensão crítica superior, $\bar{d}$, é indicada pelo comportamento logarítmico de $\beta\left(\mu_{c}\right)$; este cálculo é formalmente idêntico à determinação de $\mathcal{L}[f](s)$ para $s \sim 2 \hat{J}\left(k_{c}\right)^{+}$, apresentado no Apêndice A.6.

\section{A.3- Prova do lema A.1}

Lema A1: Em uma hiperesfera de dimensão $d \geq 2$, a integral em todo o ângulo sólido

$$
\int \frac{d \Omega}{\sqrt{\prod_{i=1}^{d}\left|\phi_{i}\right|}}
$$

em que $\left\{\phi_{i}\right\}$ é o conjunto das componentes angulares, é positiva e limitada (por $\left.\frac{\sqrt{2}}{\pi}(2 \pi)^{d}\right)$.

Prova: A transformação entre o sistema cartesiano, de dimensão $d$, de coordenadas, $\left(x_{1}, \cdots, x_{d}\right)$, e as coordenadas hiperesféricas, $\left(r, \theta_{1}, \cdots, \theta_{d-1}\right)$ (com $\theta_{i \neq d-1} \in[0, \pi]$ e $\theta_{d-1} \in[0,2 \pi)$ ), é dada por

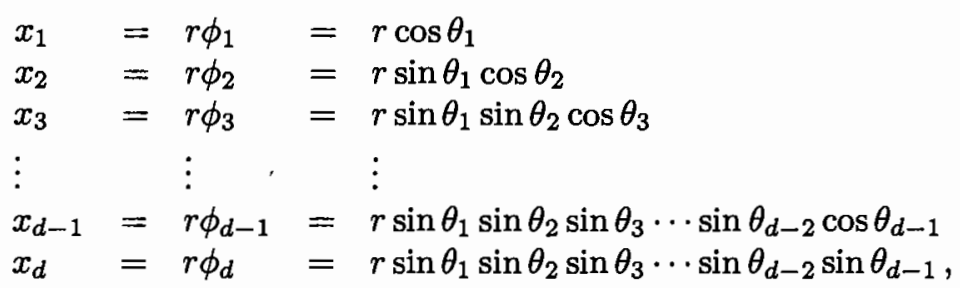


donde se obtém

$$
\prod_{i=1}^{d} \phi_{i}=\prod_{i=1}^{d-1} \cos \theta_{i} \sin ^{d-i} \theta_{i}
$$

e o jacobiano da transformação

$$
\frac{\partial\left(x_{1}, \cdots, x_{d}\right)}{\partial\left(r, \theta_{1}, \cdots, \theta_{d-1}\right)}= \begin{cases}r & , d=2 \\ r^{d-1} \prod_{i=1}^{d-2} \sin ^{d-i-1} \theta_{i} & , d \geq 3\end{cases}
$$

Logo,

$$
\int \frac{d \Omega}{\sqrt{\prod_{i=1}^{d-1}\left|\phi_{i}\right|}}=\left\{\begin{array}{ll}
\int_{0}^{2 \pi} \frac{d \theta_{1}}{\sqrt{\left|\cos \theta_{1} \sin \theta_{1}\right|}} & , d=2 \\
\int_{0}^{2 \pi} \frac{d \theta_{d-1}}{\sqrt{\left|\cos \theta_{d-1} \sin \theta_{d-1}\right|}} \prod_{i=1}^{d-2} \int_{0}^{\pi} d \theta_{i} \frac{\sin ^{\frac{d-i}{2}-1} \theta_{i}}{\sqrt{\left|\cos \theta_{i}\right|}}, & d \geq 3
\end{array} .\right.
$$

Para $\alpha \geq 0$, é imediato que

S:

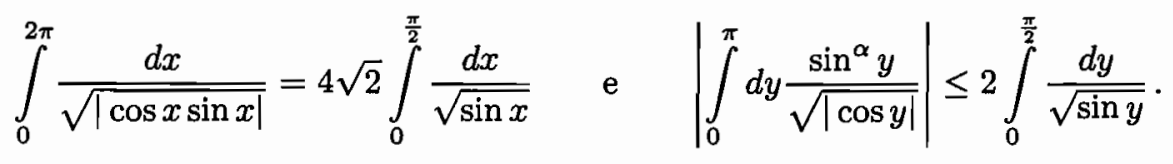

Defina, agora, a função $\kappa(x):=\frac{\sin x}{x}$ para $x \in\left(0, \frac{\pi}{2}\right]$. Nota-se que

$$
\frac{d}{d x} \kappa(x)=\frac{\cos x}{x^{2}}(x-\tan x) \leq 0,
$$

visto que no mesmo intervalo $(x-\tan x)$ é decrescente (verificável pela sua derivada) com valor máximo zero. A função $\kappa$ é, portanto, decrescente em $\left(0, \frac{\pi}{2}\right]$, ou seja, $\kappa(x) \geq \kappa\left(\frac{\pi}{2}\right)$. Como $\sin 0=0$, conclui-se que $\sin x \geq \frac{2}{\pi} x$, fato que implica

L

$$
\left|\int_{0}^{\frac{\pi}{2}} \frac{d x}{\sqrt{\sin x}}\right| \leq \pi
$$

que usado em (A.43), fornece

$$
\left|\int \frac{d \Omega}{\sqrt{\prod_{i=1}^{d}\left|\phi_{i}\right|}}\right| \leq \frac{\sqrt{2}}{\pi}(2 \pi)^{d}
$$


Por outro lado, para $d \geq 2$, tem-se $\prod_{i=1}^{d}\left|\phi_{i}\right|<1 \mathrm{e}$

$$
0<\frac{2 \pi^{\frac{d}{2}}}{\Gamma\left(\frac{d}{2}\right)}<\int \frac{d \Omega}{\sqrt{\prod_{i=1}^{d}\left|\phi_{i}\right|}}
$$

\section{A.4- Condição inicial}

Esta seção é dedicada a justificar que uma condição inicial altamente desordenada, de temperatura efetivamente infinita, corresponde a tomar $C_{k}(0,0)=1$.

A partir das transformadas de Fourier (A.5), tem-se

$$
\begin{aligned}
C_{k}(0,0) & =\left\langle\hat{S}_{k}(0) \hat{S}_{-k}(0)\right\rangle \\
& =\frac{1}{N} \sum_{x, x^{\prime} \in \Lambda_{N}}\left\langle S_{x}(0) S_{x^{\prime}}(0)\right\rangle e^{i k\left(x-x^{\prime}\right)}
\end{aligned}
$$

Na condição inicial descrita acima, as correlações e as magnetizações espontâneas são nulas; logo,

$$
\left\langle S_{x}(0) S_{x^{\prime}}(0)\right\rangle= \begin{cases}\left\langle S_{x}(0)\right\rangle\left\langle S_{x^{\prime}}(0)\right\rangle=0 & , x \neq x^{\prime} \\ \left\langle S_{x}^{2}(0)\right\rangle & , x=x^{\prime}\end{cases}
$$

Por outro lado, do vínculo esférico $C(t, t)=1$, que é válido para cada instante de tempo $t$, tem-se

$$
\begin{aligned}
N & =\sum_{x \in \Lambda_{N}}\left\langle S_{x}^{2}(0)\right\rangle \\
& =N\left\langle S_{x}^{2}(0)\right\rangle
\end{aligned}
$$

donde se conclui que $\left\langle S_{x}^{2}(0)\right\rangle=1$ e, por conseguinte, que $\left\langle S_{x}(0) S_{x^{\prime}}(0)\right\rangle=\delta_{x, x^{\prime}}$. Este resultado, quando inserido em (A.49), fornece $C_{k}(0,0)=1$.

\section{A.5- Forma assintótica de $f$}

O comportamento assintótico da função

$$
f(t):=\frac{1}{(2 \pi)^{d}} \int_{\hat{\Lambda}} d^{d} k e^{2 \hat{J}(k) t}
$$

em relação a $t$ depende diretamente da dimensionalidade e dos valores que os parâmetros $R$ e $S$ assumem. Os cálculos serão realizados para cada caso de interesse, obedecendo à classificação da seção A.2. A fim de simplificar a notação, define-se, inicialmente,

$$
g(k):=R \cos k+S \cos (2 k)
$$


que admite a expansão

$$
g(k)=g\left(q_{c}\right)-\frac{c_{2}}{2 !}\left(k-q_{c}\right)^{2}+\frac{c_{3}}{3 !}\left(k-q_{c}\right)^{3}-\frac{c_{4}}{4 !}\left(k-q_{c}\right)^{4}+\cdots,
$$

em torno de $q_{c}$, onde os coeficientes foram definidos em (A.14).

Reescrevendo (A.52) como

$$
f(t)=\left[\frac{1}{2 \pi} \int_{-\pi}^{\pi} d k e^{4 J t g(k)}\right]^{m}\left[I_{0}(4 J t)\right]^{d-m},
$$

onde $I_{0}(x)$ é a função de Bessel modificada de ordem zero em $x$, é suficiente determinar a forma assintótica de dois termos distintos.

Suponha, inicialmente, que $c_{2}>0$ e $q_{c} \neq 0, \pi$; considere $\delta t^{-\alpha} \in \mathbb{R}_{+}$, onde $\alpha \in\left(\frac{1}{3}, \frac{1}{2}\right)$ e $\delta \in \mathbb{R}_{+}$é fixo. Em dimensão um, $U_{1}^{1}\left(q_{c}, \delta t^{-\alpha}\right)=K_{1}^{1}\left(q_{c}, \delta t^{-\alpha}\right)$, e tem-se

$$
\begin{aligned}
\frac{1}{\pi} \int_{0}^{\pi} d k e^{4 J t g(k)}= & \frac{e^{4 J t g\left(q_{c}\right)}}{\pi} \int_{U_{1}^{1}\left(q_{c}, \delta t^{-\alpha}\right)} d k \exp \left[-2 J t c_{2}\left(k-q_{c}\right)^{2}+\mathcal{O}\left(t \delta^{3} t^{-3 \alpha}\right)\right]+ \\
& +\frac{1}{\pi} \int_{[0, \pi] \backslash U_{1}^{1}\left(q_{c}, \delta t^{-\alpha}\right)} d k e^{4 J t g(k)} \\
= & \frac{e^{4 J t g\left(q_{c}\right)}}{\pi} \int_{-\delta t^{-\alpha}}^{\delta t^{-\alpha}} d k e^{-2 J t c_{2} k^{2}}\left[1+\mathcal{O}\left(\delta^{3} t^{1-3 \alpha}\right)\right]+\frac{1}{\pi} \int_{[0, \pi] \backslash U_{1}^{1}\left(q_{c}, \delta t^{-\alpha}\right)} d k e^{4 J t g(k)} \\
= & \frac{2}{\sqrt{8 \pi J c_{2} t}} e^{4 J t g\left(q_{c}\right)} \operatorname{erf}\left(\delta \sqrt{2 J c_{2}} t^{1 / 2-\alpha}\right)\left[1+\mathcal{O}\left(\delta^{3} t^{1-3 \alpha}\right)\right]+\frac{1}{\pi} \int_{[0, \pi] \backslash U_{1}^{1}\left(q_{c}, \delta t^{-\alpha}\right)} d k e^{4 J t g(k)} \\
= & \frac{2}{\sqrt{8 \pi J c_{2} t}} e^{4 J t g\left(q_{c}\right)}\left[1+\mathcal{O}\left(\delta^{3} t^{1-3 \alpha}\right)\right]+\frac{1}{\pi} \int_{[0, \pi] \backslash U_{1}^{1}\left(q_{c}, \delta t^{-\alpha}\right)} d k e^{4 J t g(k)} .
\end{aligned}
$$

O último termo em (A.56) é limitado por $e^{4 J t\left[R \cos \left(\delta t^{-\alpha}\right)+S \cos \left(2 \delta t^{-\alpha}\right)\right]} ; \operatorname{logo}$,

$$
\lim _{t \rightarrow \infty} \sqrt{t} e^{-4 J t g\left(q_{c}\right)}\left[\frac{1}{\pi} \int_{0}^{\pi} d k e^{4 J t g(k)}\right]=\frac{1}{\sqrt{2 \pi J c_{2}}},
$$

ou, simbolicamete, a expansão assintótica (em relação a $t$ ) é representada por

$$
\frac{1}{\pi} \int_{0}^{\pi} d k e^{4 J t g(k)} \sim \frac{2}{\sqrt{8 \pi J c_{2}}} \frac{e^{4 J t g\left(q_{c}\right)}}{\sqrt{t}} .
$$

Procedendo-se de forma análoga para $q_{c}=0$ ou $q_{c}=\pi$, tem-se

$$
\frac{1}{\pi} \int_{0}^{\pi} d k e^{4 J t g(k)} \sim \frac{1}{\sqrt{8 \pi J} c_{2}} \frac{e^{4 J t g\left(g_{c}\right)}}{\sqrt{t}}
$$


que difere do caso anterior por um fator dois no prefator.

Para $c_{2}=0$, que é possível somente quando $q_{c}=0$ ou $q_{c}=\pi$ (se $q_{c}=\pi$, translada-se em $\pi$ a variável $k$ ), define-se novamente $\delta t^{-\alpha} \in \mathbb{R}_{+}$, onde agora $\alpha \in\left(\frac{1}{6}, \frac{1}{4}\right)$. Com esta escolha, tem-se

$$
\begin{aligned}
\frac{1}{\pi} \int_{0}^{\pi} d k e^{4 J t g(k)=} & \frac{e^{4 J t g\left(q_{c}\right)}}{\pi} \int_{0}^{\delta t^{-\alpha}} d k \exp \left[-\frac{J t}{6} c_{4} k^{4}+\mathcal{O}\left(t \delta^{6} t^{-6 \alpha}\right)\right]+ \\
& +\frac{1}{\pi} \int_{\delta t^{-\alpha}}^{\pi} d k e^{4 J t g(k)} \\
= & \frac{e^{4 J t g\left(q_{c}\right)}}{\pi} \int_{0}^{\delta t^{-\alpha}} d k e^{-\frac{J t c_{4}}{6} k^{4}}\left[1+\mathcal{O}\left(\delta^{6} t^{1-6 \alpha}\right)\right]+\frac{1}{\pi} \int_{\delta t^{-\alpha}}^{\pi} d k e^{4 J t g(k)} \\
= & \left(\frac{6}{J t c_{4}}\right)^{\frac{1}{4}} \frac{e^{4 J t g\left(q_{c}\right)}}{\pi} \int_{0}^{\delta\left(\frac{J c_{4}}{6}\right)^{1 / 4} t^{1 / 4-\alpha}} d u e^{-u^{4}}\left[1+\mathcal{O}\left(\delta^{6} t^{1-6 \alpha}\right)\right]+\frac{1}{\pi} \int_{\delta t^{-\alpha}}^{\pi} d k e^{4 J t g(k)} \\
= & \left(\frac{6}{J t c_{4}}\right)^{\frac{1}{4}} \frac{e^{4 J t g\left(q_{c}\right)}}{\pi} \Gamma\left(\frac{5}{4}\right)\left[1+\mathcal{O}\left(\delta^{6} t^{1-6 \alpha}\right)\right]+\frac{1}{\pi} \int_{\delta t^{-\alpha}}^{\pi} d k e^{4 J t g(k)}
\end{aligned}
$$

Por fim,

$$
\frac{1}{\pi} \int_{0}^{\pi} d k e^{4 J t g(k)} \sim \frac{1}{(8 \pi J)^{\frac{1}{4}}}\left(\frac{48}{\pi^{3} c_{4}}\right)^{\frac{1}{4}} \Gamma\left(\frac{5}{4}\right) \frac{e^{4 J t g\left(q_{c}\right)}}{t^{\frac{1}{4}}} .
$$

A expansão assintótica da função de Bessel de ordem zero para argumentos grandes é, mutatis mutandis, similar aos casos anteriores:

$$
I_{0}(4 J t):=\frac{1}{2 \pi} \int_{-\pi}^{\pi} d k e^{4 J t \cos k} \sim \frac{e^{4 J t}}{\sqrt{8 \pi J t}}
$$

Lembrando que

$$
\hat{J}\left(k_{c}\right)=2 J\left[m g\left(q_{c}\right)+d-m\right],
$$

basta combinar os resultados (A.58), (A.59), (A.61) e (A.62) para determinar a forma assintótica de $f$ via (A.55).

$\diamond \operatorname{Casos} 1$ e $2(m \neq 0, d-m \neq 0)$

* Caso $1\left(c_{2}>0\right)$

$$
f(t) \sim \begin{cases}(8 \pi J)^{-\frac{d}{2}} c_{2}^{-\frac{m}{2}} \frac{e^{2 \hat{J}\left(k_{c}\right) t}}{t^{\frac{d}{2}}} \quad, \quad q_{c}=0, \pi \\ 2^{m}(8 \pi J)^{-\frac{d}{2}} c_{2}^{-\frac{m}{2}} \frac{e^{2 \hat{J}\left(k_{c}\right) t}}{t^{\frac{d}{2}}} \quad, \quad q_{c} \neq 0, \pi\end{cases}
$$


* Caso $2\left(c_{2}=0\right)$

$$
f(t) \sim(8 \pi J)^{-\frac{2 d-m}{4}}\left(\frac{48}{\pi^{3}}\right)^{\frac{m}{4}} \Gamma\left(\frac{5}{4}\right)^{m}\left|c_{4}\right|^{-\frac{m}{4}} \frac{e^{2 \hat{J}\left(k_{c}\right) t}}{t^{\frac{2 d-m}{4}}}
$$

$\diamond \operatorname{Casos} 3$ e $4(m \neq 0, d-m=0)$

* Caso $3\left(c_{2}>0\right)$

$$
f(t) \sim\left\{\begin{array}{cc}
(8 \pi J)^{-\frac{d}{2}} c_{2}^{-\frac{d}{2}} \frac{e^{2 \hat{J}\left(k_{c}\right) t}}{t^{\frac{d}{2}}} & , \quad q_{c}=0, \pi \\
2^{d}(8 \pi J)^{-\frac{d}{2}} c_{2}^{-\frac{d}{2}} \frac{e^{2 \hat{J}\left(k_{c}\right) t}}{t^{\frac{d}{2}}} & , \quad q_{c} \neq 0, \pi
\end{array}\right.
$$

* Caso $4\left(c_{2}=0\right)$

$$
f(t) \sim(8 \pi J)^{-\frac{d}{4}}\left(\frac{48}{\pi^{3}}\right)^{\frac{d}{4}} \Gamma\left(\frac{5}{4}\right)^{d}\left|c_{4}\right|^{-\frac{d}{4}} \frac{e^{2 \hat{J}\left(k_{c}\right) t}}{t^{\frac{d}{4}}}
$$

$\diamond$ Caso $5(m=0, d-m \neq 0)$

$$
f(t) \sim(8 \pi J)^{-\frac{d}{2}} \frac{e^{2 \hat{J}\left(k_{c}\right) t}}{t^{\frac{d}{2}}}
$$

Os resultados acima indicam que

$$
f(t) \sim K_{p} \frac{e^{2 \hat{J}\left(k_{c}\right) t}}{t^{\gamma_{p}}},
$$

onde $K_{p}=K(R, S, m, d)$ e $\gamma_{p}=\gamma(R, S, m, d)$ assumem valores distintos em cada caso.

\section{A.6- Transformada de Laplace de $f(|\epsilon| \ll 1)$}

As dimensões críticas do modelo, calculadas na seção A.2, são informações importantes para determinar o comportamento assintótico da transformada de Laplace $\mathcal{L}[f](s)$ para $s \sim 2 \hat{J}\left(k_{c}\right)^{+}$, que é essencial para chegar à expansão assintótica de $\psi(t)$ para grandes valores de $t$. A partir da definição de $f,($ A.52), o objetivo aqui é o cálculo de

$$
\mathcal{L}[f](s)=\int_{[-\pi, \pi]^{d}} \frac{d^{d} k}{(2 \pi)^{d}} \frac{1}{\epsilon+2 \hat{J}\left(k_{c}\right)-2 \hat{J}(k)},
$$

onde $\epsilon:=\epsilon(s)=s-2 \hat{J}\left(k_{c}\right)$. 
Será considerada a transformada de Laplace (para $\epsilon \sim 0$ ) quando $m=0$ e $d-m \neq 0$ (o caso 5 , na seção anterior), que é a situação mais simples, para ilustrar o procedimento padrão de cálculo de $\mathcal{L}[f](s)$ para $\epsilon \sim 0$. Os demais casos, embora tecnicamente mais árduos, são resolvidos mutatis mutandis.

$\diamond$ Caso $5(m=0, d-m \neq 0)$

A equação a ser considerada é

$$
\mathcal{L}[f](s)=\int_{[-\pi, \pi]^{d}} \frac{d^{d} k}{(2 \pi)^{d}} \frac{1}{\epsilon+2 \hat{J}\left(k_{c}\right)-2 \hat{J}(k)},
$$

com $k_{c}=0,2 \hat{J}\left(k_{c}\right)=4 J d$ e $2 \hat{J}(k)=4 J \sum_{i=1}^{d} \cos k_{i}$. Recorrendo, novamente, ao conjunto $U_{1}^{d}\left(k_{c}, \delta\right)$, definido em (A.17), tem-se

$$
\begin{aligned}
\mathcal{L}[f](s)= & \int_{U_{1}^{d}\left(k_{c}, \delta\right)} \frac{d^{d} k}{(2 \pi)^{d}} \frac{1}{\epsilon+2 \hat{J}\left(k_{c}\right)-\left[2 \hat{J}\left(k_{c}\right)-2 J \sum_{i=1}^{d} k_{i}^{2}+\mathcal{O}\left(\delta^{4}\right)\right]}+ \\
& +\int_{[-\pi, \pi]^{d} \backslash U_{1}^{d}\left(k_{c}, \delta\right)} \frac{d^{d} k}{(2 \pi)^{d}} \frac{1}{\epsilon+2 \hat{J}\left(k_{c}\right)-2 \hat{J}(k)} .
\end{aligned}
$$

No segundo membro, reescrevendo o primeiro termo em coordenadas (hiper)esféricas e notando que para $\delta>0$ o segundo termo é analítico em $\epsilon$, tem-se

$$
\begin{aligned}
\mathcal{L}[f](s) & =\frac{2 \pi^{\frac{d}{2}}}{\Gamma\left(\frac{d}{2}\right)(2 \pi)^{d}} \int_{0}^{\delta} \frac{d k k^{d-1}}{\epsilon+2 J k^{2}}+\mathcal{O}\left(\delta^{2}\right)+\sum_{j=1}^{\infty}(-1)^{j-1} A_{j}^{\prime}(d, \delta) \epsilon^{j-1} \\
& =\frac{\epsilon^{\frac{d-2}{2}}}{(8 \pi J)^{\frac{d}{2}} \Gamma\left(\frac{d}{2}\right)} \int_{0}^{\frac{2 J \delta^{2}}{e}} \frac{d k k^{\frac{d}{2}-1}}{k+1}+\mathcal{O}\left(\delta^{2}\right)+\sum_{j=1}^{\infty}(-1)^{j-1} A_{j}^{\prime}(d, \delta) \epsilon^{j-1}
\end{aligned}
$$

onde

$$
A_{j}^{\prime}(d, \delta):=\int_{[-\pi, \pi]^{d} \backslash U_{1}^{d}\left(k_{c}, \delta\right)} \frac{d^{d} k}{(2 \pi)^{d}} \frac{1}{\left[2 \hat{J}\left(k_{c}\right)-2 \hat{J}(k)\right]^{j}} .
$$

Define-se, também,

$$
A_{j}(d):=\int_{[-\pi, \pi]^{d}} \frac{d^{d} k}{(2 \pi)^{d}} \frac{1}{\left[2 \hat{J}\left(k_{c}\right)-2 \hat{J}(k)\right]^{j}} .
$$

Note que, do vínculo esférico (2.15) e de (A.75), tem-se

$$
A_{1}=\frac{1}{2 T_{c}}
$$


Para uma análise detalhada do comportamento assintótico de $\mathcal{L}[f](s)$, examinar-se-ão, separadamente, os termos $\int_{0}^{2 J \delta^{2} / \epsilon} \frac{d k k^{\frac{d}{2}-1}}{k+1}$ e $A_{j}^{\prime}(d, \delta) \epsilon^{j-1}$.

Para dimensões pares, $d=2 q, q \in \mathbb{N}$, e escolhendo $\epsilon \ll \delta^{2} \ll 1$, tem-se

$$
\begin{aligned}
\int_{0}^{\frac{2 J \delta^{2}}{\epsilon}} \frac{d k k^{\frac{2 q}{2}-1}}{k+1} & =\int_{1}^{1+\frac{2 J \delta^{2}}{\epsilon}} \frac{d u}{u} \sum_{j=0}^{q-1}\left(\begin{array}{c}
q-1 \\
j
\end{array}\right)(-1)^{q-1-j} u^{j} \\
& =\left\{\begin{array}{ll}
-\ln \epsilon+\mathcal{O}\left(\ln \delta^{2}\right) & , q=1 \\
(-1)^{q} \ln \epsilon+2(-1)^{q-1} \ln \delta+\frac{1}{q-1}\left(\frac{2 J \delta^{2}}{\epsilon}\right)^{q-1}+\mathcal{O}\left(\left(\frac{\delta^{2}}{\epsilon}\right)^{q-2}\right), & q>1
\end{array} .\right.
\end{aligned}
$$

Delimitando a escolha de $\delta$ para valores que satisfazem $\epsilon \ll \delta^{2} \ll \epsilon|\ln \epsilon|^{\frac{1}{q-1}} \ll 1^{12}$, tem-se

$$
\int_{0}^{\frac{2 J \delta^{2}}{\epsilon}} \frac{d k k^{\frac{2 q}{2}-1}}{k+1} \sim\left\{\begin{array}{ll}
-\ln \epsilon+\mathcal{O}\left(\ln \delta^{2}\right) & , q=1 \\
(-1)^{q} \ln \epsilon+\mathcal{O}\left(\left(\frac{\delta^{2}}{\epsilon}\right)^{q-1}\right), & q>1
\end{array} .\right.
$$

Para dimensões que não são pares, tem-se (Apêndice A.7)

$$
\begin{aligned}
\int_{0}^{\frac{2 J \delta^{2}}{\epsilon}} \frac{d k k^{\frac{d}{2}-1}}{k+1} & =\Gamma_{g}\left(\frac{d}{2}\right)-\overline{\Gamma_{g}}\left(\frac{d}{2}\right) \\
& =\Gamma\left(\frac{d}{2}\right) \Gamma\left(1-\frac{d}{2}\right)-\frac{2(2 J)^{\frac{d-2}{2}}}{2-d}\left(\frac{\epsilon}{\delta^{2}}\right)^{\left|\frac{d-2}{2}\right|}\left[1+\mathcal{O}\left(\frac{\epsilon}{\delta^{2}}\right)\right]
\end{aligned}
$$

sendo que $\Gamma_{g}$ e $\overline{\Gamma_{g}}$ são definidos e tratados na seção seguinte. A partir de (A.73) e (A.79), tem-se, lembrando que $\epsilon \ll \delta^{2} \ll 1$,

$$
\begin{aligned}
\mathcal{L}[f](s)= & \sum_{j=1}^{\infty}(-1)^{j-1} A_{j}^{\prime}(d, \delta) \epsilon^{j-1}+\mathcal{O}\left(\delta^{2}\right)+ \\
& + \begin{cases}(8 \pi J)^{-\frac{d}{2}} \Gamma\left(1-\frac{d}{2}\right) \epsilon^{-\frac{2-d}{2}}+\mathcal{O}\left(\delta^{-(2-d)}\right) & , d<2 \\
(8 \pi J)^{-1}(-\ln \epsilon)+\mathcal{O}\left(-\ln \delta^{2}\right) & , d=2 \\
(8 \pi J)^{-\frac{d}{2}} \Gamma\left(1-\frac{d}{2}\right) \epsilon^{\frac{d-2}{2}}+\mathcal{O}\left(\delta^{d-2}\right) & , \quad 2<d<4 \\
(8 \pi J)^{-2} \epsilon \ln \epsilon+\mathcal{O}\left(\delta^{2}\right) & , d=4 \\
\mathcal{O}\left(\delta^{d-2}\right) & , d>4\end{cases}
\end{aligned}
$$

\footnotetext{
${ }^{12}$ Uma escolha possivel seria $\delta^{2}=\epsilon \sqrt{|\ln \epsilon|^{(q-1)^{-1}}}$.
} 
Reunindo (A.80) com (A.93) (vide seção seguinte), que fornece o comportamento da série acima, $\sum_{j=1}^{\infty}(-1)^{j-1} A_{j}^{\prime}(d, \delta) \epsilon^{j-1}$, tem-se

$$
\mathcal{L}[f](s)= \begin{cases}(8 \pi J)^{-\frac{d}{2}} \Gamma\left(1-\frac{d}{2}\right) \epsilon^{-\frac{2-d}{2}}+\mathcal{O}\left(\delta^{-(2-d)}\right) & , d<2 \\ (8 \pi J)^{-1}(-\ln \epsilon)+\mathcal{O}\left(-\ln \delta^{2}\right) & , d=2 \\ A_{1}(d)-(8 \pi J)^{-\frac{d}{2}}\left|\Gamma\left(1-\frac{d}{2}\right)\right| \epsilon^{\frac{d-2}{2}}+\mathcal{O}\left(\delta^{d-2}\right) & , \quad 2<d<4 \\ A_{1}(d)-(8 \pi J)^{-2}(-\epsilon \ln \epsilon)+\mathcal{O}\left(\delta^{2}\right) & , d=4 \\ A_{1}(d)-A_{2}(d) \epsilon+\mathcal{O}\left(\delta^{d-4}\right) & , \quad d>4\end{cases}
$$

lembrando que $\epsilon \ll \delta^{2} \ll \epsilon|\ln \epsilon|^{\frac{2}{d-2}} \ll 1$.

\section{A.7- Funções $\Gamma_{g}, \overline{\Gamma_{g}}$ e $A_{j}^{\prime}$}

Esta seção preocupará em estudar as funções $\Gamma_{g}, \overline{\Gamma_{g}}$ e $A_{j}^{\prime}$, que apareceram na seção anterior na determinação de $\mathcal{L}[f](s)$ para $s \sim 2 \hat{J}\left(k_{c}\right)$. Estas três funções são úteis para desenvolver os cálculos necessários para o caso 5, embora não os sejam necessariamente nos outros quatro casos - onde outras funções, similares àquelas que aqui aparecem, precisam ser definidas. Entretanto, como já dito, o procedimento técnico nos cinco casos é o mesmo.

\section{A.7.1- $\Gamma_{g}$}

A função

$$
\Gamma_{g}(p):=\int_{0}^{\infty} d x \frac{x^{p-1}}{x+1}
$$

é, a.priori, definida para $p \in(0,1)$ e, no plano complexo, tem um ramo que, convenientemente, é colocado sobre a semi - reta $[0, \infty)$. O contorno é mostrado abaixo. 
APÊNDICE A

51

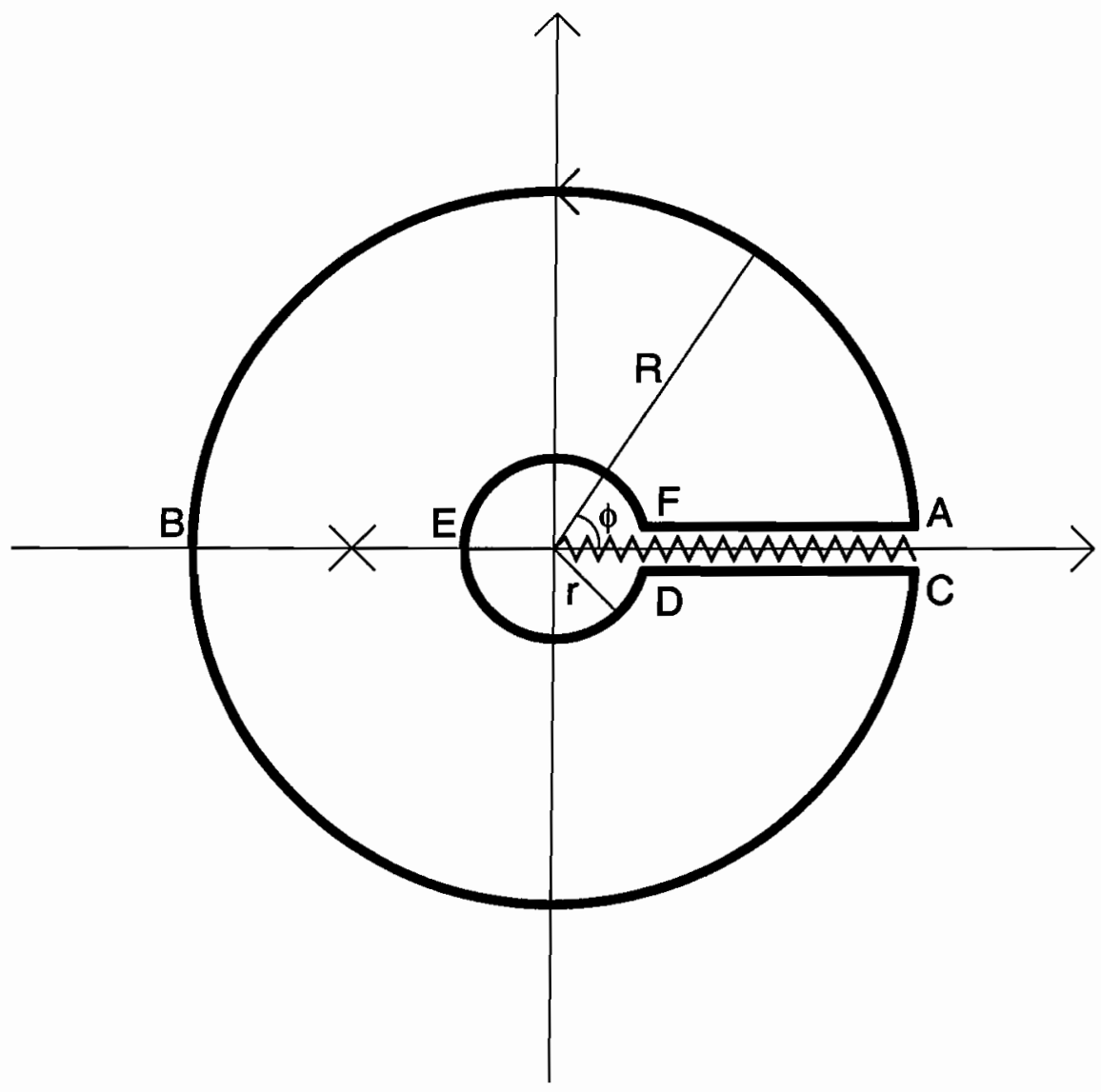

Figura A1: Contorno para $\Gamma_{g}$.

O pólo simples -1 determina o resíduo, que é $e^{i \pi(p-1)} . \mathrm{O}$ teorema dos resíduos assegura que

$$
\begin{aligned}
2 \pi i e^{i \pi(p-1)}= & \int_{r}^{R} d\left(x e^{i 0}\right) \frac{\left(x e^{i 0}\right)^{p-1}}{x e^{i 0}+1}+\int_{0}^{2 \pi} d\left(R e^{i \phi}\right) \frac{\left(R e^{i \phi}\right)^{p-1}}{R e^{i \phi}+1}+ \\
& +\int_{R}^{r} d\left(x e^{i 2 \pi}\right) \frac{\left(x e^{i 2 \pi}\right)^{p-1}}{x e^{i 2 \pi}+1}+\int_{2 \pi}^{0} d\left(r e^{i \phi}\right) \frac{\left(r e^{i \phi}\right)^{p-1}}{r e^{i \phi}+1},
\end{aligned}
$$

que, nos limites $r \rightarrow 0$ e $R \rightarrow \infty$, assume a forma

$$
2 \pi i e^{i \pi(p-1)}=\int_{0}^{\infty} d x \frac{x^{p-1}}{x+1}\left[1-e^{i 2 \pi(p-1)}\right] \Leftrightarrow \int_{0}^{\infty} d x \frac{x^{p-1}}{x+1}=\frac{2 \pi i e^{i \pi p} e^{-i \pi}}{1-e^{i 2 \pi p} e^{-i 2 \pi}}=\frac{2 \pi i}{e^{i \pi p}-e^{-i \pi p}}
$$

finalmente,

$$
\int_{0}^{\infty} d x \frac{x^{p-1}}{x+1}=\frac{\pi}{\sin (p \pi)}, \quad p \in(0,1)
$$


Existe, porém, uma forma mais adequada para $\Gamma_{g}(p)$, que pode ser obtida mediante o seguinte cálculo:

$$
\Gamma(p) \Gamma(1-p)=\int_{0}^{\infty} d t e^{-t} t^{p-1} \int_{0}^{\infty} d s e^{-s} s^{1-p-1}=\int_{0}^{\infty} d t \int_{0}^{\infty} d s e^{-(t+s)} t^{-1}\left(\frac{t}{s}\right)^{p}
$$

com a mudança de variáveis $u=t+s$ e $v=\frac{t}{s}$, é imediato que

$$
\Gamma(p) \Gamma(1-p)=\Gamma_{g}(p) \quad, \quad p \in(0,1),
$$

que é um resultado de maior interesse. A função $\Gamma_{g}$ é definida somente no aberto $(0,1)$; no entanto, através da equação funcional (A.87) (ou (A.85)), é possível estendê-la analiticamente para toda a reta real, exceto nos pontos inteiros. Seu gráfico é obtido pela equação (A.85) ou (A.87) para $p \in \mathbb{R} \backslash \mathbb{Z}$ :

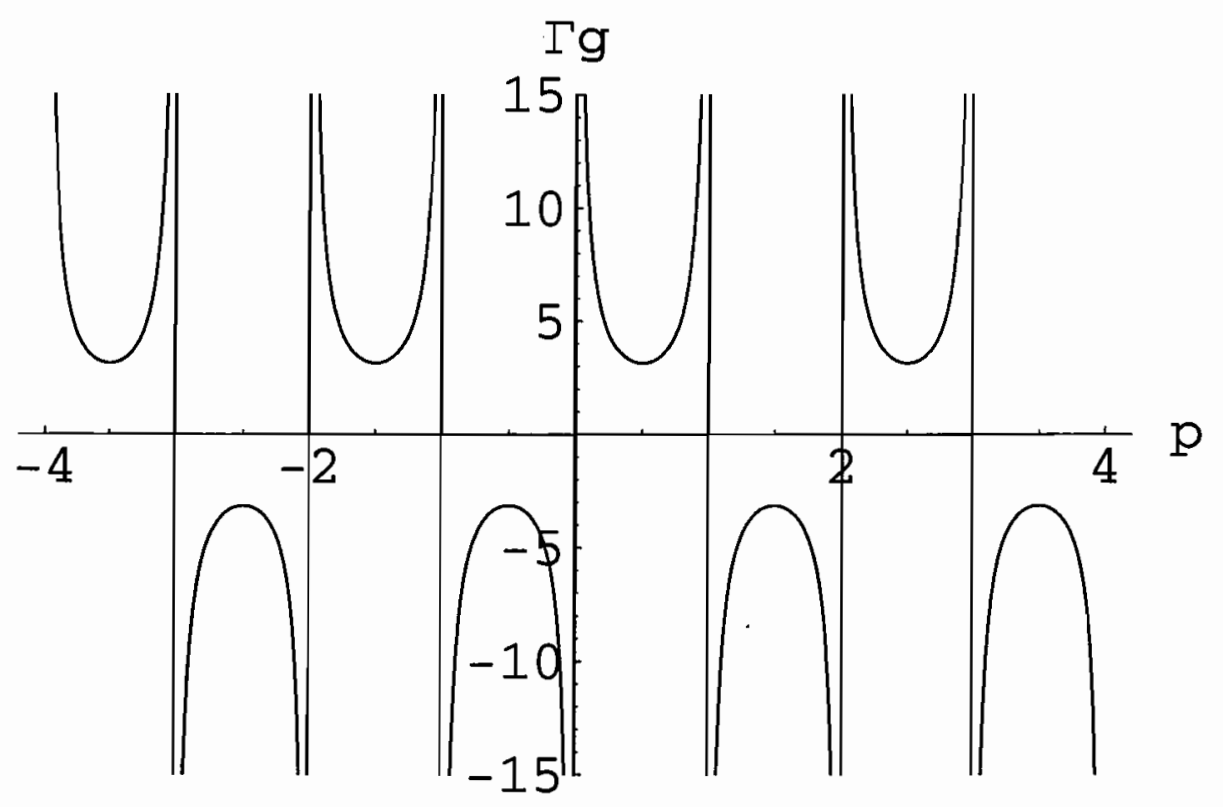

Figura A2: Gráfico $\Gamma_{g}(p) \times p$.

\section{A.7.2- $\overline{\Gamma_{g}}$}

Novamente, admitindo inicialmente que $p \in(0,1)$, a integral

$$
\overline{\Gamma_{g}}(p):=\int_{R}^{\infty} d x \frac{x^{p-1}}{x+1}
$$

com $R \gg 1$ converge, fornecendo, em um cálculo simples, 


$$
\overline{\Gamma_{g}}(p)=\sum_{j=0}^{\infty}(-1)^{j} \frac{R^{p-1-j}}{j+1-p}=\frac{1}{1-p} \frac{1}{R^{|1-p|}}\left[1+\mathcal{O}\left(R^{-1}\right)\right]
$$

A continuação analítica do intervalo $(0,1)$ para $\mathbb{R} \backslash \mathbb{Z}$ é feita através da equação (A.89).

\section{A.7.3- $A_{j}^{\prime}$}

* Caso $5(m=0, d-m \neq 0)$

Da seção A.2, é imediato que $A_{1}(d)=A_{1}^{\prime}(d, 0)$ converge para $d>2$. Com o mesmo procedimento adotado, verifica-se que $A_{j}(d)$ é finito para $d>2 j$. Neste caso,

$$
\begin{aligned}
A_{j}^{\prime}(d, \delta) & =A_{j}(d)-\int_{U_{1}^{d}\left(k_{c}, \delta\right)} \frac{d^{d} k}{(2 \pi)^{d}} \frac{1}{\left[2 \hat{J}\left(k_{c}\right)-2 \hat{J}(k)\right]^{j}} \\
& =A_{j}(d)-\int_{U_{1}^{d}\left(k_{c}, \delta\right)} \frac{d^{d} k}{(2 \pi)^{d}} \frac{1}{\left[2 J \sum_{i=1}^{d} k_{i}^{2}\right]^{j}}+\mathcal{O}\left(\delta^{2}\right) \\
& =A_{j}(d)-\frac{2 \pi^{\frac{d}{2}}}{(2 \pi)^{d} \Gamma\left(\frac{d}{2}\right)(2 J)^{j}} \int_{0}^{\delta} d k k^{d-1-2 j}+\mathcal{O}\left(\delta^{2}\right) \\
& =A_{j}(d)+\mathcal{O}\left(\delta^{d-2 j}\right)+\mathcal{O}\left(\delta^{2}\right), \quad d>2 j .
\end{aligned}
$$

Para $d \leq 2 j$, quando a integral $A_{j}(d)$ diverge, deve-se explicitar o comportamento assintótico de $\epsilon^{j-1} A_{j}^{\prime}(d, \delta)$. Provar-se-á, agora, que $\cos x \geq 1-\frac{x^{2}}{2}$ em toda a reta real; esta desigualdade será útil mais adiante.

Defina, inicialmente, $g(x):=\cos x-1+\frac{x^{2}}{2}, x \in \mathbb{R} ;$ mostrar-se-á que $g(x) \geq 0$, a igualdade sendo válida somente no ponto $x=0$. Pela paridade de $g$, é suficiente verificar a asserção somente na semireta não-negativa. Como $g(0)=0$, deve-se provar que $g(x)>0$ em $\mathbb{R}_{+}$. Para $x>2$, verifica-se que $g(x) \geq-1-1+\frac{x^{2}}{2}>0$, restando analisar somente o intervalo $(0,2)$, visto que esta desigualdade estendese no ponto $x=2$. Como $\frac{d^{2} g(x)}{d x^{2}}=-\cos x+1>0 \mathrm{em}(0,2)$, a função $\frac{d g}{d x}$ é crescente nesse domínio; pela continuidade de $\frac{d g}{d x}$, tem-se $0=\frac{d g(0)}{d x}<\frac{d g(x)}{d x}$ em $(0,2)$, mostrando que $g$ é crescente nesse intervalo e, pela sua continuidade, $0=g(0)<g(x)$, completando a prova. A desigualdade $\cos x \geq 1-\frac{x^{2}}{2}$ será aplicada na seguinte majoração ${ }^{13}$ :

\footnotetext{
${ }^{13}$ É importante destacar que esta desigualdade é válida em toda a reta real, e não é fruto de uma expansão de Mac Laurin.
} 


$$
\begin{aligned}
\left|\epsilon^{j-1} A_{j}^{\prime}(d, \delta)\right| & \leq\left|\epsilon_{[-\pi, \pi]^{j-1} \backslash U_{1}^{d}\left(k_{c}, \delta\right)} \frac{d^{d} k}{(2 \pi)^{d}} \frac{1}{\left[4 J d-4 J\left(d-\sum_{i=1}^{d} \frac{k_{i}^{2}}{2}\right)\right]^{j}}\right| \\
& =\left.\frac{\epsilon^{j-1}}{(2 J)^{j}(2 \pi)^{d}}\right|_{[-\pi, \pi]^{d} \backslash U_{1}^{d}\left(k_{c}, \delta\right)} \frac{d^{d} k}{\left(\sum_{i=1}^{d} k_{i}^{2}\right)^{j}} \mid \\
& \leq \frac{\epsilon^{j-1}}{(2 J)^{j}(2 \pi)^{d}} \frac{2 \pi^{\frac{d}{2}}}{\Gamma\left(\frac{d}{2}\right)}\left|\int_{\delta}^{\pi \sqrt{d}} d k k^{d-1-2 j}\right| \\
& = \begin{cases}\frac{\epsilon^{\frac{d-2}{2}}}{(8 \pi J)^{\frac{d}{2}} \Gamma\left(\frac{d}{2}\right)}\left|\ln \left(\pi^{2} d\right)-\ln \delta^{2}\right|=\frac{\epsilon^{j-1}}{(8 \pi J)^{j} \Gamma(j)}\left|\ln \left(2 \pi^{2} j\right)-\ln \delta^{2}\right|, & , d=2 j \\
\frac{\epsilon^{j-1}}{(2 J)^{j}(2 \pi)^{d}} \frac{2 \pi^{\frac{d}{2}}}{\Gamma\left(\frac{d}{2}\right)}\left|\frac{(\pi \sqrt{d})^{d-2 j}}{d-2 j}+\frac{\left(\delta^{2}\right)^{-\frac{2 j-d}{2}}}{2 j-d}\right| & d<2 j\end{cases}
\end{aligned}
$$

Em suma,

$$
\left|\epsilon^{j-1} A_{j}^{\prime}(d, \delta)\right| \leq \begin{cases}\frac{1}{(8 \pi J)^{j} \Gamma(j)} \epsilon^{j-1}\left|\ln \delta^{2}\right|+\mathcal{O}\left(\epsilon^{j-1}\right) & , d=2 j \\ \frac{2 \pi^{\frac{d}{2}}}{(2 J)^{j}(2 \pi)^{d} \Gamma\left(\frac{d}{2}\right)(2 j-d)} \delta^{d-2}\left(\frac{\epsilon}{\delta^{2}}\right)^{j-1}+\mathcal{O}\left(\epsilon^{j-1}\right) & , d<2 j\end{cases}
$$

De (A.90) e (A.92), chega-se a

$$
\sum_{j=1}^{\infty}(-1)^{j-1} A_{j}^{\prime}(d, \delta) \epsilon^{j-1}= \begin{cases}\mathcal{O}\left(\delta^{-(2-d)}\right) & , d<2 \\ \mathcal{O}\left(-\ln \delta^{2}\right) & , d=2 \\ A_{1}(d)+\mathcal{O}\left(\delta^{d-2}\right) & , 2<d<4 \\ A_{1}(d)+\mathcal{O}\left(\delta^{2}\right) & , d=4 \\ A_{1}(d)-A_{2}(d) \epsilon+\mathcal{O}\left(\delta^{d-4}\right) & , d>4\end{cases}
$$

\section{A.8- Comportamento assintótico de $\psi$}

\section{A.8.1- Considerações gerais}

Esta seção é dedicada a determinar a forma assintótica de 


$$
\psi(t)=\frac{1}{2 \pi i} \int_{\sigma-i \infty}^{\sigma+i \infty} d s e^{s t} \mathcal{L}[\psi](s), \quad \mathcal{L}[\psi](s):=\frac{\mathcal{L}[f](s)}{1-2 T \mathcal{L}[f](s)}
$$

para $t \sim \infty$. A partir da transformada de Laplace de $f$,

$$
\mathcal{L}[f](s)=\int_{0}^{\infty} d t e^{-s t} \int_{[-\pi, \pi]^{d}} \frac{d^{d} k}{(2 \pi)^{d}} e^{2 \hat{J}(k) t}=\int_{[-\pi, \pi]^{d}} \frac{d^{d} k}{(2 \pi)^{d}} \frac{1}{s-2 \hat{J}(k)},
$$

tomando $s>2 \hat{J}\left(k_{c}\right)$ para $s \in \mathbb{R}$, a continuação analítica do último membro de (A.95) indica que o plano complexo de $s$ tem um corte no segmento $\left[\inf _{k \in\{-\pi, \pi]^{d}}\{2 \hat{J}(k)\}, 2 \hat{J}\left(k_{c}\right)\right]$. Nota-se, ainda, que para $s \in \mathbb{R}, \mathcal{L}[f](s)$ é monotonicamente decrescente em $s$, sendo $\mathcal{L}[f](s) \in\left[0, \frac{\beta_{c}}{2}\right]$, onde $\frac{\beta_{c}}{2}$ é infinito quando não há ocorrência de transição de fase.

Defina $P:=\left\{y \in \mathbb{C}: y\right.$ é pólo de $\left.e^{s t} \mathcal{L}[\psi](s)\right\}$ e $\bar{p}:=\sup _{y \in P}\{\Re y\}$. As dinâmicas supercrítica, crítica e subcrítica, correspondem a um "quench" a uma temperatura $T$ respectivamente superior, igual e inferior à crítica $T_{c}$.

\section{A.8.2- Regime supercrítico}

Pela propriedade de monotonicidade de $\mathcal{L}[f](s)$, o denominador de (A.94) percorre o intervalo [1 $\left.\frac{T}{T_{c}}, 1\right]$, atingindo cada ponto uma única vez. Como $T>T_{c}$, o integrando de (A.94) apresenta um (único) pólo isolado, que será denotado por $\tau_{e q}^{-1}$.

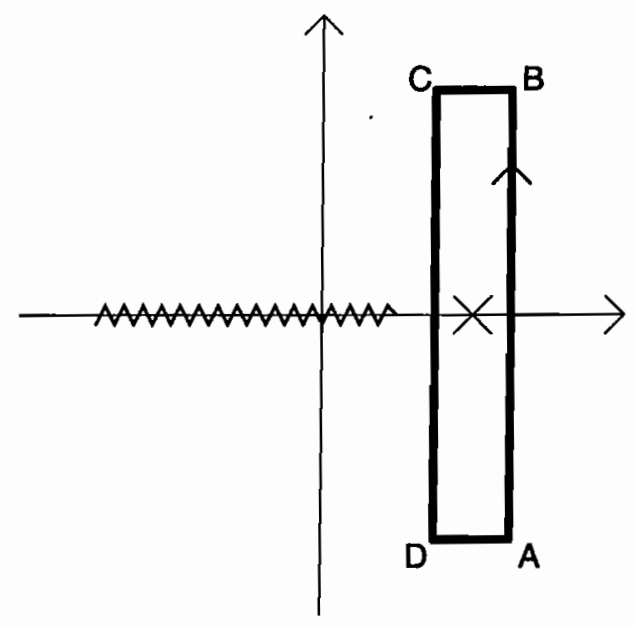

Figura A3: Contorno para integração.

No contorno indicado na figura A.3, seja $A B C D$ um retângulo de vértices $c \pm i R$ e $\sigma \pm i R$, onde $c<\bar{p}<\sigma$; escolha $2 \hat{J}\left(k_{c}\right)<c<\bar{p}<\sigma$. Segundo o teorema dos resíduos, 


$$
\begin{aligned}
2 \pi i \operatorname{Res} e^{s t} \mathcal{L}[\psi](s)= & \int_{\substack{A B \cup B C \cup C D \cup D A \\
\sigma+i R}} d s e^{s t} \mathcal{L}[\psi](s) \\
= & \int_{\sigma-i R}^{c} d s e^{s t} \mathcal{L}[\psi](s)+e^{i R t} \int_{\sigma}^{c} d y e^{y t} \mathcal{L}[\psi](y+i R)+ \\
& +i e^{c t} \int_{R}^{-R} d y e^{i y t} \mathcal{L}[\psi](c+i y)+e^{-i R t} \int_{c}^{\sigma} d y e^{y t} \mathcal{L}[\psi](y-i R) .
\end{aligned}
$$

Verifica-se que $\lim _{R \rightarrow \infty}|\mathcal{L}[\psi](y \pm i R)|=0$, para $y \in[c, \sigma]$, e que o terceiro termo é $\mathcal{O}\left(e^{c t}\right)$, sendo desprezível para $t \sim \infty$ em relação ao primeiro (que é $2 \pi i \psi(t)$ para $R \rightarrow \infty$ ), que é $\mathcal{O}\left(e^{\sigma t}\right)$. Logo, no regime supercrítico e para tempos assintoticamente grandes $t \sim \infty$, chega-se a

$$
\psi(t) \sim \operatorname{Res} e^{s t} \mathcal{L}[\psi](s)=-\frac{1}{4 T^{2}} \frac{1}{\frac{\partial}{\partial s} \mathcal{L}[\psi]\left(\tau_{e q}^{-1}\right)} e^{\frac{t}{\tau_{e q}}}
$$

$\mathrm{O}$ valor de $\tau_{e q}$ pode ser estimado em regiões próximas à temperatura crítica. À medida que a temperatura $T$ é admitida como sendo próxima a $T_{c}^{+}$, o pólo aproxima-se da extemidade do corte em $s=2 \hat{J}\left(k_{c}\right)$ (atingindo-o quando $T=T_{c}$, que é o caso crítico, assunto da próxima subseção). Para $T$ suficientemente perto de $T_{c}, T \sim T_{c}^{+}$, a função $\mathcal{L}[\psi]$, na determinação do resíduo

$$
\operatorname{Res} e^{s t} \mathcal{L}[\psi](s) \sim \lim _{s \rightarrow 2 \hat{J}\left(k_{c}\right)^{+}}\left[s-2 \hat{J}\left(k_{c}\right)\right] e^{s t} \mathcal{L}[\psi](s), \quad\left(T \sim T_{c}^{+}\right)
$$

pode ser substituída pela sua forma assintótica,

$$
\mathcal{L}[\psi](s) \sim \begin{cases}\frac{F_{p} g_{p}}{\epsilon^{-\alpha_{p}}-2 T F_{p} g_{p}} & , d<d_{c} \\ \frac{F_{p}(-\ln \epsilon)}{1-2 T F_{p}(-\ln \epsilon)} & , d=d_{c} \\ \frac{A_{1}-F_{p} g_{p} \epsilon^{\alpha_{p}}}{1-2 T A_{1}+2 T F_{p}\left|g_{p}\right| \epsilon^{\alpha_{p}}} & , \quad d_{c}<d<\bar{d} \\ \frac{A_{1}-F_{p}(-\epsilon \ln \epsilon)}{1-2 T A_{1}+2 T F_{p}(-\epsilon \ln \epsilon)} & , d=\bar{d} \\ \frac{A_{1}-A_{2} \epsilon}{1-2 T A_{1}+2 T A_{2} \epsilon} & , d>\bar{d}\end{cases}
$$

calculada através de (A.94) e (A.81). Aqui, deve-se lembrar que $\epsilon:=s-2 \hat{J}\left(k_{c}\right)$ e que $T_{c}=0$ para $d \leq d_{c}$, quando então não há transiição de fase. A expansão assintótica (A.99) fornece uma estimativa dos pólos para $T \sim T_{c}^{+}$, que é 


$$
\tau_{e q}^{-1} \sim \begin{cases}2 \hat{J}\left(k_{c}\right)+\left(2 T F_{p} g_{p}\right)^{-\frac{1}{\alpha_{p}}} & , d<d_{c} \\ 2 \hat{J}\left(k_{c}\right)+\exp \left(-\frac{1}{2 T F_{p}}\right) & , d=d_{c} \\ 2 \hat{J}\left(k_{c}\right)+\left[-\frac{1}{2 T F_{p}\left|g_{p}\right|}\left(1-\frac{T}{T_{c}}\right)\right]^{\frac{1}{\alpha_{p}}} & , \quad d_{c}<d<\bar{d}, \\ 2 \hat{J}\left(k_{c}\right)+\epsilon^{*} & , d=\bar{d} \\ 2 \hat{J}\left(k_{c}\right)+\left(-\frac{1}{2 T A_{2}}\right)\left(1-\frac{T}{T_{c}}\right) & , d>\bar{d}\end{cases}
$$

onde $\epsilon^{*}$ é a raíz de

$$
\epsilon \ln \epsilon=\frac{1}{2 T F_{p}}\left(1-\frac{T}{T_{c}}\right)
$$

tal que $|\epsilon| \ll 1$ - esta última condição é necesária para especificar a solução de (A.101), que não é única.

\section{A.8.3- Regime crítico}

O pólo de $\mathcal{L}[\psi](s)$, simples e isolado no regime supercrítico, coincide com o ponto $2 \hat{J}\left(k_{c}\right)$, que é um dos extremos do corte. Seja $c<2 \hat{J}\left(k_{c}\right)<\sigma$; tomando o contorno da figura $A 4($ a), com $A=\sigma-i R$, $B=\sigma+i R, C=c-i R$ e $I=c+i R$, a aplicação do teorema de Cauchy indica que

$$
\int_{\sigma-i R}^{\sigma+i R} d s e^{s t} \mathcal{L}[\psi](s)=\int_{I B} d s e^{s t} \mathcal{L}[\psi](s)+\int_{A C} d s e^{s t} \mathcal{L}[\psi](s)+\int_{C D \cup D E \cup E F G \cup G H \cup H I} d s e^{s t} \mathcal{L}[\psi](s)
$$
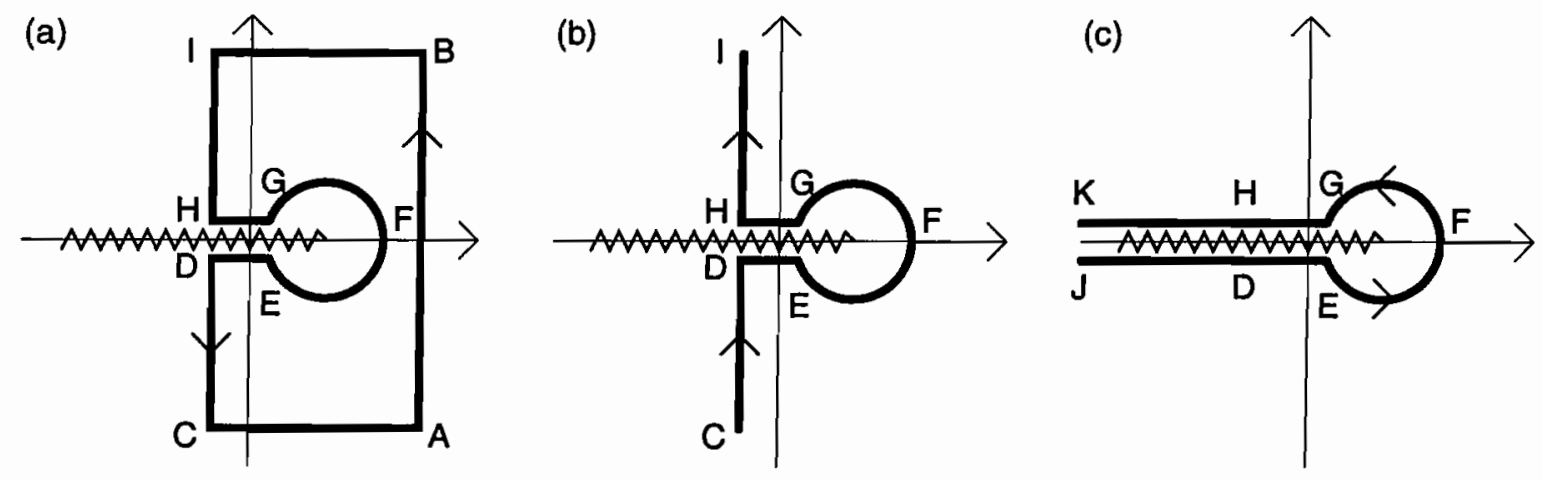

Figura A4: Contornos para integração.

Como $\lim _{R \rightarrow \infty}|\mathcal{L}[\psi](y \pm i R)|=0$, para $y \in[c, \sigma]$, as integrais nos caminhos $I B$ e $A C$ anulamse no limite $R \rightarrow \infty$, que deve ser tomado. A determinação do primeiro membro de (A.102) (que é $2 \pi i f(t)$ quando $R \rightarrow \infty$ ) fica condicionada ao cálculo da terceira integral do segundo membro, que é 
representada pelo contorno na figura $\mathrm{A} 4$ (b). O teorema de Cauchy permite distorcer os caminhos $C D$ e $H I$, e a integral de interesse passa a ser aquela calculada no contorno mostrado na figura A4(c) no limite $R \rightarrow \infty$,

$$
\psi(t)=\frac{1}{2 \pi i} \int_{\sigma-i \infty}^{\sigma+i \infty} d s e^{s t} \mathcal{L}[\psi](s)=\lim _{R \rightarrow \infty} \frac{1}{2 \pi i} \int_{J E F G K} d s e^{s t} \mathcal{L}[\psi](s)
$$

Para tempos suficientemente grandes, a contribuição do caminho $E F G$, que é $\mathcal{O}\left(e^{2 \hat{J}\left(k_{c}\right) t}\right)$, é dominante sobre aquelas provenientes dos caminhos $J D$ e $H K$, que fornecem contribuições $\mathcal{O}\left(e^{c t}\right)$. Logo, substituir $\mathcal{L}[f](s)$ por sua expansão assintótica (2.33) para calcular $\mathcal{L}[\psi](s)$ é uma operação legítima, com correções desprezíveis para $t \sim \infty$.

Com as observações acima, pode-se finalmente explicitar, para tempos longos no regime crítico, o comportamento assintótico de $\psi$. Este comportamento depende da dimensionalidade do sistema.

Se $d \in\left(d_{c}, \bar{d}\right), \mathcal{L}[f](s) \sim A_{1}-F_{p}\left|g_{p}\right| \epsilon^{\alpha_{p}}$, conforme (2.33). Então, $\mathcal{L}[\psi](s) \sim \frac{A_{l}^{2}}{F_{p} \mid g_{p}} \epsilon^{-\alpha_{p}}$ e

$$
\psi(t) \sim \frac{A_{1}^{2}}{F_{p}\left|g_{p}\right|} \frac{1}{2 \pi i} \int_{J E F G K} d s e^{s t}\left(s-2 \hat{J}\left(k_{c}\right)\right)^{-\alpha_{p}} .
$$

Com a mudança de variável $\left(s-2 \hat{J}\left(k_{c}\right)\right) \rightarrow s$, é simples notar que a integral acima é a função gama com o contorno de Hankel. Portanto,

$$
\psi(t) \sim \frac{A_{1}^{2}}{F_{p}\left|g_{p}\right|} \frac{t^{\alpha_{p}-1} e^{2 \hat{J}\left(k_{c}\right) t}}{\Gamma\left(\alpha_{p}\right)}, \quad\left(d_{c}<d<\bar{d}\right)
$$

Se $d=\bar{d}, \mathcal{L}[f](s) \sim A_{1}-F_{p}(-\epsilon \ln \epsilon)$ e, assim, $\mathcal{L}[\psi](s) \sim-\frac{A_{1}^{2}}{F_{p} \epsilon \ln \epsilon}$. Novamente, o contorno a ser utilizado é aquele indicado pela figura A.4(c). Pode-se mostrar, facilmente, que a contribuição proveniente do arco $E F G$ é nula (levando o seu raio a zero); então,

$$
\begin{aligned}
\psi(t) & \sim-\frac{A_{1}}{2 T_{c} F_{p}} \frac{e^{2 \hat{J}\left(k_{c}\right) t}}{2 \pi i}\left[\int_{\infty}^{0} \frac{d\left(r e^{-i \pi}\right) e^{t r e^{-i \pi}}}{r e^{-i \pi}(\ln r-i \pi)}+\int_{0}^{\infty} \frac{d\left(r e^{i \pi}\right) e^{t r e^{i \pi}}}{r e^{i \pi}(\ln r+i \pi)}\right] \\
& =\frac{A_{1}}{2 T_{c} F_{p}} e^{2 \hat{J}\left(k_{c}\right) t} \int_{0}^{\infty} \frac{d r e^{-r t}}{r\left(\ln ^{2} r+\pi^{2}\right)}
\end{aligned}
$$

Recorrendo a uma mudança de variável $r \rightarrow e^{\pi r}$, e em seguida integrando por partes, tem-se 


$$
\begin{aligned}
\psi(t) & \sim \frac{A_{1}}{2 T_{c} F_{p}} \frac{e^{2 \hat{J}\left(k_{c}\right) t}}{\pi} \int_{-\infty}^{\infty} \frac{d r \exp \left(-t e^{\pi r}\right)}{r^{2}+1} \\
& =\frac{A_{1}}{2 T_{c} F_{p}} \frac{e^{2 \hat{\jmath}\left(k_{c}\right) t}}{\pi}\left\{\left.\exp \left(-t e^{\pi r}\right) \tan ^{-1} r\right|_{-\infty} ^{\infty}+\pi t \int_{-\infty}^{\infty} d r \tan ^{-1} r \exp \left(\pi r-t e^{\pi r}\right)\right\} \\
& =\frac{A_{1}}{2 T_{\mathrm{c}} F_{p}} \frac{e^{2 \hat{J}\left(k_{c}\right) t}}{\pi}\left\{\frac{\pi}{2}-\int_{0}^{\infty} d u e^{-u} \tan ^{-1}\left[\frac{1}{\pi} \ln \left(\frac{t}{u}\right)\right]\right\} \\
& =\frac{A_{1}}{2 T_{c} F_{p}} \frac{e^{2 \hat{J}\left(k_{c}\right) t}}{\pi}\left\{\frac{\pi}{2}-\int_{0}^{\infty} d u e^{-u}\left[\frac{\pi}{2}-\frac{\pi}{\ln \left(\frac{t}{u}\right)}+\mathcal{O}\left(\ln ^{-3} t\right)\right]\right\} \\
& =\frac{A_{1}}{2 T_{c} F_{p}} \frac{e^{2 \hat{J}\left(k_{c}\right) t}}{\ln t}\left[\int_{0}^{\infty} d u \frac{e^{-u}}{1-\frac{\ln u}{\ln t}}+\mathcal{O}\left(\ln { }^{-4} t\right)\right]
\end{aligned}
$$

A aplicação $\frac{e^{-u}}{1-\frac{\ln u}{\ln t}}$, enquanto função de $u$, é, em quase toda parte, definida no intervalo $(0, \infty)$, e a integral em

$$
A(t):=\int_{0}^{\infty} d u \frac{e^{-u}}{1-\frac{\ln u}{\ln t}}
$$

deve ser entendida com sendo o valor principal de Cauchy

$$
A(t):=\text { v.p. } \int_{0}^{\infty} d u \frac{e^{-u}}{1-\frac{\ln u}{\ln t}}
$$

O último resultado para se chegar à expansão assintótica de $\psi$ para $d=\bar{d}$ é

Lema A2: $\lim _{t \rightarrow \infty} A(t)=1$ com a função $A$ definida em (A.109).

A prova é apresentada no Apêndice A.9.

Para $d=\bar{d}$, então, o lema A2 garante que

$$
\psi(t) \sim \frac{A_{1}}{2 T_{c} F_{p}} \frac{e^{2 \hat{J}\left(k_{c}\right) t}}{\ln t}
$$

Para $d>\bar{d}, \mathcal{L}[f](s) \sim A_{1}-A_{2} \epsilon$ e, portanto, $\mathcal{L}[\psi](s) \sim \frac{A_{1}^{2}}{A_{2}\left(s-2 \hat{J}\left(k_{c}\right)\right)} \cdot$ Então,

$$
\psi(t) \sim \frac{A_{1}^{2}}{A_{2}} \frac{1}{2 \pi i} \int_{J E F G K} d s \frac{e^{s t}}{s-2 \hat{J}\left(k_{c}\right)}
$$


com a contribuição dos caminhos $H K$ e $J D$ sendo desprezíveis frente àquela próxima do ponto $s=$ $2 \hat{J}\left(k_{c}\right)$ quando $t \sim \infty$. Fazendo o raio da curva $E F G$ tender a zero, é imediato que

$$
\psi(t) \sim \frac{A_{1}^{2}}{A_{2}} e^{2 \hat{J}\left(k_{c}\right) t}, \quad(d>\bar{d})
$$

Em suma,

$$
\psi(t) \sim\left\{\begin{array}{ll}
\frac{A_{1}^{2}}{F_{p}|g| \Gamma\left(\alpha_{p}\right)} \frac{e^{2 \hat{J}\left(k_{c}\right) t}}{t^{1-\alpha_{p}}} & d_{c}<d<\bar{d} \\
\frac{A_{1}}{2 T_{c} F_{p}} \frac{e^{2 \hat{J}\left(k_{c}\right) t}}{\ln t} & d=\bar{d} \\
\frac{A_{1}^{2}}{A_{2}} e^{2 \hat{J}\left(k_{c}\right) t} & d>\bar{d}
\end{array} .\right.
$$

\section{A.8.4- Regime subcrítico}

No regime subcrítico, para $s \sim 2 \hat{J}\left(k_{c}\right)$, tem-se $\mathcal{L}[f](s) \sim A_{1}-h\left(\left[s-2 \hat{J}\left(k_{c}\right]\right)\right)$, onde $h$ é uma função contínua para $s \geq 2 \hat{J}\left(k_{c}\right)$ e $h(0)=0$. A forma de $h$ pode ser encontrada em (2.33). Um cálculo simples indica que

$$
\mathcal{L}[\psi](s)=\frac{1}{M_{e q}^{4}} \mathcal{L}[f](s)-\frac{T A_{1}}{M_{e q}^{4} T_{c}}+\mathcal{O}\left(\left[h\left(s-2 \hat{J}\left(k_{c}\right)\right]^{2}\right), \quad s \sim 2 \hat{J}\left(k_{c}\right)\right.
$$

onde

$$
M_{e q}^{2}:=1-\frac{T}{T_{c}}
$$

Com os mesmos argumentos apresentados no regime crítico, e usando os mesmos contornos da figura A4 (irrelevância dos caminhos $B I$ e $A C$ ), tem-se, para tempos grandes,

$$
\psi(t)=\frac{1}{M_{e q}^{4}} \int_{J E F G K} d s e^{s t} \mathcal{L}[f](s)+\mathcal{O}\left(\left[h\left(s-2 \hat{J}\left(k_{c}\right)\right]^{2}\right)\right.
$$

Com um raciocínio análogo, pode-se representar a integral acima (primeiro termo do segundo membro de (A.116)) pelo caminho $A B$, chegando-se a

$$
\psi(t) \sim \frac{1}{M_{e q}^{4}} \mathcal{L}^{-1} \mathcal{L}[f](t)=\frac{f(t)}{M_{e q}^{4}}
$$




\section{A.9- Prova do lema A2}

Demonstrar-se-á, agora, o

Lema A2: $\lim _{t \rightarrow \infty} A(t)=1$ com a função $A$ definida em (A.109).

Prova: Assumindo $t \gg e$, a integral em

$$
A(t)=\mathrm{v} \cdot \mathrm{p} \cdot \int_{0}^{\infty} d u \frac{e^{-u}}{1-\frac{\ln u}{\ln t}}=A^{(1)}(t)+A^{(2)}(t)+A^{(3)}(t)
$$

é dividida em três partes, sendo que

$$
A^{(1)}(t):=\int_{0}^{\frac{1}{\ln t}} d u \frac{e^{-u}}{1-\frac{\ln u}{\ln t}}=\ln t \int_{0}^{\frac{1}{\ln t}} d u \frac{e^{-u}}{\ln t+|\ln u|}
$$

Então,

$$
\left|A^{(1)}(t)\right| \leq \frac{\ln t}{\ln t+\ln \ln t} \int_{0}^{\frac{1}{\ln t}} d u e^{-u}=\frac{\ln t}{\ln t+\ln \ln t}\left(1-e^{-\frac{1}{\ln t}}\right)=\frac{\ln t}{\ln t+\ln \ln t}\left[\frac{1}{\ln t}+\mathcal{O}\left(\ln ^{-2} t\right)\right]
$$

ri: $\quad \lim _{t \rightarrow \infty} A^{(1)}(t)=0$.

O segundo termo, $A^{(2)}(t)$, é responsável pela contribuição não nula do comportamento assintótico de $A(t)$ :

$$
A^{(2)}(t):=\int_{\frac{1}{\ln t}}^{\ln t} d u \frac{e^{-u}}{1-\frac{\ln u}{\ln t}}=\int_{\frac{1}{\ln t}}^{\ln t} d u e^{-u}[1+o(1)]=e^{-\frac{1}{\ln t}}-e^{-\ln t}+o(1)
$$

logo, $\lim _{t \rightarrow \infty} A^{(2)}(t)=1$.

Resta mostrar que o terceiro termo, $A^{(3)}(t)$, é nulo para $t \rightarrow \infty$. Dividindo-o em três termos,

$f^{x}$

$$
A^{(3)}(t):=\mathrm{v} \cdot \mathrm{p} \cdot \int_{\ln t}^{\infty} d u \frac{e^{-u}}{1-\frac{\ln u}{\ln t}}=A^{(3 \alpha)}(t)+A^{(3 b)}(t)+A^{(3 c)}(t)
$$

mostrar-se-á que cada um deles é nulo no limite $t \rightarrow \infty$.

O primeiro termo,

$$
A^{(3 a)}(t):=\int_{\ln t}^{t\left(1-\frac{1}{\ln t}\right)} d u \frac{e^{-u}}{1-\frac{\ln u}{\ln t}}=-t \ln t \int_{\frac{\ln t}{t}}^{1-\frac{1}{\ln t}} d u \frac{e^{-t u}}{\ln u}
$$


admite a majoração

$$
\begin{aligned}
\left|A^{(3 a)}(t)\right| & \leq t \ln t \int_{\frac{\ln t}{t}}^{1-\frac{1}{\ln t}} d u \frac{e^{-t u}}{|\ln u|} \leq \frac{t \ln t}{\left|\ln \left(1-\frac{1}{\ln t}\right)\right|} \int_{\frac{\ln t}{t}}^{1-\frac{1}{\ln t}} d u e^{-t u} \\
& =\frac{t \ln t}{\left|\frac{1}{\ln t}+\mathcal{O}\left(\frac{1}{\ln ^{2} t}\right)\right|} \frac{1}{t}\left[\frac{1}{t}-e^{-t\left(1-\frac{1}{\ln t}\right)}\right]
\end{aligned}
$$

donde $\lim _{t \rightarrow \infty} A^{(3 a)}(t)=0$.

O segundo termo,

$$
A^{(3 b)}(t):=\text { v.p. } \int_{t\left(1-\frac{1}{\ln t}\right)}^{t\left(1+\frac{1}{\ln t}\right)} d u \frac{e^{-u}}{1-\frac{\ln u}{\ln t}}=-t \ln t \text { v.p. } \int_{1-\frac{1}{\ln t}}^{1+\frac{1}{\ln t}} d u \frac{e^{-t u}}{\ln u}
$$

por ser majorado por

$$
\begin{aligned}
\left|A^{(3 b)}(t)\right| & \leq t \ln t \text { v.p. } \int_{1-\frac{1}{\ln t}}^{1+\frac{1}{\ln t}} d u \frac{e^{-t u}}{|\ln u|} \\
& \leq t e^{-t\left(1-\frac{1}{\ln t}\right)} \ln t \text { v.p. } \int_{1-\frac{1}{\ln t}}^{1+\frac{1}{\ln t}} d u \frac{1}{|\ln u|} \\
& =t e^{-t\left(1-\frac{1}{\ln t}\right)} \ln t \text { v.p. } \int_{-\frac{1}{\ln t}}^{\frac{1}{\ln t}} \frac{d u}{|\ln (1+u)|} \\
& =t e^{-t\left(1-\frac{1}{\ln t}\right)} \ln t \text { v.p. } \int_{-\frac{1}{\ln t}}^{\frac{1}{\ln t}} \frac{d u}{|u|}\left[1+\frac{|u|}{2}+\mathcal{O}\left(u^{2}\right)\right] \\
& =t e^{-t\left(1-\frac{1}{\ln t}\right)} \ln t\left[\frac{1}{\ln t}+o\left(\ln { }^{-1} t\right)\right]
\end{aligned}
$$

de onde é imediato que $\lim _{t \rightarrow \infty} A^{(3 b)}(t)=0$.

Por fim, o terceiro termo,

$$
A^{(3 c)}(t):=\int_{t\left(1+\frac{1}{\ln t}\right)}^{\infty} d u \frac{e^{-u}}{1-\frac{\ln u}{\ln t}}=-t \ln t \int_{1+\frac{1}{\ln t}}^{\infty} d u \frac{e^{-t u}}{\ln u}
$$

também tende a zero no limite $t \rightarrow \infty$. Com efeito,

$$
\left|A^{(3 c)}(t)\right| \leq \frac{t \ln t}{\ln \left(1+\frac{1}{\ln t}\right)} \int_{1+\frac{1}{\ln t}}^{\infty} d u e^{-t u}=\frac{t \ln t}{\frac{1}{\ln t}+\mathcal{O}\left(\ln ^{-2} t\right)} \frac{e^{-t\left(1+\frac{1}{\ln t}\right)}}{t}
$$


implica $\lim _{t \rightarrow \infty} A^{(3 c)}(t)=0$, o que completa a prova.

\section{A.10- Três equações auxiliares}

Três equações serão de grande utilidade na determinação dos comportamentos assintóticos das funções de dois tempos:

(i) $\frac{1}{2 T_{c}}=\int_{0}^{\infty} d t e^{-2 \hat{J}\left(k_{c}\right) t} f(t)$

A equação (i) é a definição de temperatura crítica; basta substituir a definiçãa de $f(t)$ e efetuar a integração.

(ii) $\frac{1}{2 T}=\int_{0}^{\infty} d t e^{-\frac{t}{\tau_{e q}}} f(t) \quad\left(T>T_{c}\right)$

De (A.94), e $\tau_{e q}^{-1}$ sendo o pólo simples de $\mathcal{L}[\psi](s)$, tem-se

$$
\mathcal{L}[f]\left(\tau_{e q}^{-1}\right)=\frac{1}{2 T}
$$

Logo,

$$
\int_{0}^{\infty} d t e^{-\frac{t}{\tau_{e q}}} f(t)=\lim _{s \rightarrow \tau_{e q}^{-1}} \int_{0}^{\infty} d t e^{-s t} f(t)=\lim _{s \rightarrow \tau_{e q}^{-1}} \mathcal{L}[f](s)=\frac{1}{2 T}
$$

que é o resultado desejado.

(iii) $\frac{1}{2 T_{c} M_{e q}^{2}}=\int_{0}^{\infty} d t e^{-2 \hat{J}\left(k_{c}\right) t} \psi(t) \quad\left(T<T_{c}\right)$

1.

De forma geral, para $T<T_{c}$, tem-se $\mathcal{L}[f](s) \sim A_{1}+h\left(s-2 \hat{J}\left(k_{c}\right)\right)$ para $s \sim 2 \hat{J}\left(k_{c}\right)^{+}$, onde $h$ é uma função contínua de $s-2 \hat{J}\left(k_{c}\right)$, sendo $h(0)=0$. Logo, por essa expansão e recorrendo a (A.94),

$$
\lim _{s \rightarrow 2 \hat{J}\left(k_{c}\right)^{+}} \int_{0}^{\infty} d t e^{-s t} \psi(t)=\lim _{s \rightarrow 2 \hat{J}\left(k_{c}\right)^{+}} \mathcal{L}[\psi](s)=\lim _{s \rightarrow 2 \hat{J}\left(k_{c}\right)^{+}} \frac{\mathcal{L}[f](s)}{1-2 T \mathcal{L}[f](s)}=\frac{A_{1}}{1-2 T A_{1}}
$$

$\mathrm{O}$ resultado segue de $A_{1}=\frac{1}{2 T_{c}}$. 


\section{A.11- Autocorrelação, função resposta e coeficiente de flutuação - dissipação}

A autocorrelação (2.30),

$$
\begin{aligned}
C\left(t, t^{\prime}\right) & =\frac{1}{\sqrt{\psi(t) \psi\left(t^{\prime}\right)}}\left[f\left(\frac{t+t^{\prime}}{2}\right)+2 T \int_{0}^{t^{\prime}} d y f\left(\frac{t+t^{\prime}}{2}-y\right) \psi(y)\right] \\
& =\frac{1}{\sqrt{\psi\left(t^{\prime}+\tau\right) \psi\left(t^{\prime}\right)}}\left[f\left(t^{\prime}+\frac{\tau}{2}\right)+2 T \int_{0}^{t^{\prime}} d y f\left(t^{\prime}-y+\frac{\tau}{2}\right) \psi(y)\right]
\end{aligned}
$$

pode ser escrita como

$$
\begin{aligned}
C\left(t, t^{\prime}\right) & =\frac{1}{\sqrt{\psi\left(t^{\prime}+\tau\right) \psi\left(t^{\prime}\right)}}\left[\psi\left(t^{\prime}+\frac{\tau}{2}\right)-2 T \int_{t^{\prime}}^{t^{\prime}+\frac{\tau}{2}} d y f\left(t^{\prime}-y+\frac{\tau}{2}\right) \psi(y)\right] \\
& =\frac{1}{\sqrt{\psi\left(t^{\prime}+\tau\right) \psi\left(t^{\prime}\right)}}\left[\psi\left(t^{\prime}+\frac{\tau}{2}\right)-2 T \int_{0}^{\frac{\tau}{2}} d y f(y) \psi\left(t^{\prime}-y+\frac{\tau}{2}\right)\right]
\end{aligned}
$$

mediante a equação (2.26),

$$
\psi(b)=f(b)+2 T \int_{0}^{b} d y f(b-y) \psi(y)
$$

tomada no ponto $b=t^{\prime}+\frac{\tau}{2}$.

A autocorrelação, a função resposta,

$$
R\left(t, t^{\prime}\right)=f\left(\frac{\tau}{2}\right) \sqrt{\frac{\psi\left(t^{\prime}\right)}{\psi(t)}},
$$

e o coeficiente de flutuação - dissipação,

$$
X\left(t, t^{\prime}\right)=\frac{T R\left(t, t^{\prime}\right)}{\partial_{t^{\prime}} C\left(t, t^{\prime}\right)},
$$

apresentam comportamentos distintos dependendo dos regimes de tempo e temperatura. Seguindo a ordem estabelecida no capítulo 2, o cálculo destas funções será dividido em três blocos, correspondendo cada um deles a um regime de temperatura. Dentro de cada bloco, as distintas escalas de tempo determinarão diferentes comportamentos dessas três grandezas para as dinâmicas crítica e subcrítica.

A notação

$$
x:=\frac{t}{t^{\prime}}
$$

será utilizada. 


\section{A.11.1- Dinâmica supercrítica}

Pela forma assintótica (A.97) e usando a equação (A.130), chega-se, para $t^{\prime} \sim \infty$, a

$$
\begin{aligned}
C\left(t, t^{\prime}\right) & \sim \frac{1}{e^{\left(t^{\prime}+\frac{\tau}{2}\right) \tau_{e q}^{-1}}}\left[e^{\left(t^{\prime}+\frac{\tau}{2}\right) \tau_{e q}^{-1}}-2 T \int_{0}^{\frac{\tau}{2}} d y f(y) e^{\left(t^{\prime}+\frac{\tau}{2}-y\right) \tau_{e q}^{-1}}\right] \\
& =1-2 T \int_{0}^{\frac{\tau}{2}} d y f(y) e^{-\frac{y}{\tau_{e q}}}=1-2 T \int_{0}^{\infty} d y f(y) e^{-\frac{y}{\tau_{e q}}}+2 T \int_{\frac{\tau}{2}}^{\infty} d y f(y) e^{-\frac{y}{\tau_{e q}}} \\
& =T \int_{\tau}^{\infty} d y f\left(\frac{y}{2}\right) e^{-\frac{y}{2 \tau_{e q}}}
\end{aligned}
$$

onde na última passagem recorreu-se à equação auxiliar (ii) (seção A.10) para, em seguida, realizar a transformação de variáveis $y \rightarrow \frac{y}{2}$.

Quanto à função resposta, é imediato de (A.132) e (A.97) que

$$
R\left(t, t^{\prime}\right) \sim f\left(\frac{\tau}{2}\right) e^{-\frac{\tau}{2 \tau_{e q}}}
$$

De (A.135) e (A.136), o teorema flutuação - dissipação,

$$
X\left(t, t^{\prime}\right) \sim T f\left(\frac{\tau}{2}\right) e^{-\frac{\tau}{2 \tau_{e q}}}\left[-\frac{\partial}{\partial \tau} T \int_{\tau}^{\infty} d y f\left(\frac{y}{2}\right) e^{-\frac{y}{2 \tau_{e q}}}\right]^{-1}=1
$$

é obedecido.

\section{A.11.2- Dinâmica crítica}

O caso crítico é a única das três dinâmicas onde a dimensionalidade deve ser considerada na caracterização dos comportamentos assintóticos das funções de dois pontos. Deve-se observar que a ocorrência de transição é requerida, e portanto $d>d_{c}$. Os cálculos serão divididos em três partes conforme a dimensão.

\section{A.11.2.1- $d_{c}<d<\bar{d}$}

Se $d_{c}<d<\bar{d}$, sabe-se que $\psi(t) \sim \frac{e^{2 \hat{j}\left(k_{c}\right) t}}{t^{1-\alpha_{p}}}$, de (A.105). No regime estacionário, em que $1 \sim \tau \ll t^{\prime}$, usa-se (A.130) para se chegar a 


$$
\begin{aligned}
C\left(t, t^{\prime}\right) & \sim \frac{\left[\left(t^{\prime}+\tau\right) t^{\prime}\right]^{\frac{1-\alpha_{p}}{2}}}{e^{\hat{J}\left(k_{c}\right)\left(2 t^{\prime}+\tau\right)}}\left[\frac{e^{\hat{J}\left(k_{c}\right)\left(2 t^{\prime}+\tau\right)}}{\left(t^{\prime}+\frac{\tau}{2}\right)^{1-\alpha_{p}}}-2 T_{c} \int_{0}^{\frac{\tau}{2}} d y f(y) \frac{e^{\hat{J}\left(k_{c}\right)\left(2 t^{\prime}+\tau-2 y\right)}}{\left(t^{\prime}+\frac{\tau}{2}-y\right)^{1-\alpha_{p}}}\right] \\
& =\left[\frac{4\left(1+\frac{\tau}{t^{\prime}}\right)}{\left(2+\frac{\tau}{t^{\prime}}\right)^{2}}\right]^{\frac{1-\alpha_{p}}{2}}\left[1-2 T_{c} \int_{0}^{\frac{\tau}{2}} \frac{d y f(y) e^{-2 \hat{J}\left(k_{c}\right) y}}{\left(1-\frac{y}{t^{\prime}+\frac{\tau}{2}}\right)^{1-\alpha_{p}}}\right] \\
& \sim 1-2 T_{c} \int_{0}^{\frac{\tau}{2}} d y f(y) e^{-2 \hat{J}\left(k_{c}\right) y} \\
& =C_{e q, c}(\tau),
\end{aligned}
$$

onde a equação auxiliar (i) (seção A.10) foi invocada e

$$
C_{e q, c}(\tau):=T_{c} \int_{\tau}^{\infty} d y f\left(\frac{y}{2}\right) e^{-\hat{J}\left(k_{c}\right) y}
$$

A função resposta, sendo

$$
\begin{aligned}
R\left(t, t^{\prime}\right) & \sim f\left(\frac{\tau}{2}\right) \sqrt{\frac{e^{2 \hat{J}\left(k_{c}\right) t^{\prime} / t^{1-\alpha_{p}}}}{e^{2 \hat{J}\left(k_{c}\right) t} / t^{1-\alpha_{p}}}} \\
& =f\left(\frac{\tau}{2}\right) \frac{e^{-\hat{J}\left(k_{c}\right) \tau}}{\left(1+\frac{\tau}{t^{\prime}}\right)^{1-\alpha_{p}}} \\
& \sim f\left(\frac{\tau}{2}\right) e^{-\hat{J}\left(k_{c}\right) \tau}
\end{aligned}
$$

também é uma função somente de $\tau$, e o teorema flutuação - dissipação, $X\left(t, t^{\prime}\right) \sim 1$, é assintoticamente obedecido.

No caso "aging", de (A.130), tem-se

$$
\begin{aligned}
C\left(t, t^{\prime}\right) & \sim \frac{\left[\left(t^{\prime}+\tau\right) t^{\prime}\right]^{\frac{1-\alpha_{p}}{2}}}{e^{\hat{J}\left(k_{c}\right)\left(2 t^{\prime}+\tau\right)}}\left[\frac{e^{\hat{J}\left(k_{c}\right)\left(2 t^{\prime}+\tau\right)}}{\left(t^{\prime}+\frac{\tau}{2}\right)^{1-\alpha_{p}}}-2 T_{c} \int_{0}^{\frac{\tau}{2}} d y \frac{e^{\hat{J}\left(k_{c}\right)\left(2 t^{\prime}+\tau-2 y\right)}}{\left(t^{\prime}+\frac{\tau}{2}-y\right)^{1-\alpha_{p}}} f(y)\right] \\
& =\frac{\left[\left(t^{\prime}+\tau\right) t^{\prime}\right]^{\frac{1-\alpha_{p}}{2}}}{\left(t^{\prime}+\frac{\tau}{2}\right)^{1-\alpha_{p}}}\left[1-2 T_{c} \int_{0}^{\frac{\tau}{2}} \frac{d y f(y) e^{-2 \hat{J}\left(k_{c}\right) y}}{\left(1-\frac{y}{t^{\prime}+\frac{\tau}{2}}\right)^{1-\alpha_{p}}}\right] .
\end{aligned}
$$

Escolhendo um $\delta>0$ tal que $2 / \tau \ll \delta \ll 1^{14}$, dividindo a integral em (A.141) em duas partes, vem, lembrando de (A.69) e que $\alpha_{p}=\gamma_{p},-1$ (equação (2.34)),

\footnotetext{
${ }^{14}$ Por exemplo, $\delta=2 / \sqrt{\tau}$
} 


$$
\begin{aligned}
\int_{0}^{\frac{\tau}{2}} \frac{d y f(y) e^{-2 \hat{J}\left(k_{c}\right) y}}{\left(1-\frac{y}{t^{\prime}+\frac{\tau}{2}}\right)^{1-\alpha_{p}}} & =\int_{0}^{\frac{\delta \tau}{2}} \frac{d y f(y) e^{-2 \hat{J}\left(k_{c}\right) y}}{\left(1-\frac{y}{t^{\prime}+\frac{\tau}{2}}\right)^{1-\alpha_{p}}}+\int_{\frac{\delta \tau}{2}}^{\frac{\tau}{2}} \frac{d y f(y) e^{-2 \hat{J}\left(k_{c}\right) y}}{\left(1-\frac{y}{t^{\prime}+\frac{\tau}{2}}\right)^{1-\alpha_{p}}} \\
& \sim \int_{0}^{\frac{\delta \tau}{2}} d y f(y) e^{-2 \hat{J}\left(k_{c}\right) y}[1+\mathcal{O}(\delta)]+K_{p} \int_{\frac{\delta \tau}{2}}^{\frac{\tau}{2}} \frac{d y}{y^{\gamma_{p}}\left(1-\frac{y}{t^{\prime}+\frac{\tau}{2}}\right)^{1-\alpha_{p}}} \\
& =\int_{0}^{\frac{\delta \tau}{2}} d y f(y) e^{-2 \hat{J}\left(k_{c}\right) y}[1+\mathcal{O}(\delta)]+K_{p} \int_{\frac{\delta \tau}{2}}^{\frac{\tau}{2}} \frac{d y}{y^{\gamma_{p}}\left(1-\frac{y}{t^{\prime}+\frac{\tau}{2}}\right)^{2-\gamma_{p}}} \\
& =\int_{0}^{\frac{\delta \tau}{2}} d y f(y) e^{-2 \hat{J}\left(k_{c}\right) y}[1+\mathcal{O}(\delta)]+K_{p} \int_{\frac{2}{\tau}}^{\frac{2}{\delta \tau}} \frac{d z}{\left(z-\frac{1}{t^{\prime}+\frac{\tau}{2}}\right)^{2-\gamma_{p}}}
\end{aligned}
$$

onde, na última linha, recorreu-se à mudança de variável $z=1 / y$. Invocando (A.69) e a relação auxiliar (i) (seção A.10),

$$
\begin{aligned}
\int_{0}^{\frac{\tau}{2}} \frac{d y f(y) e^{-2 \hat{J}\left(k_{c}\right) y}}{\left(1-\frac{y}{t^{\prime}+\frac{\tau}{2}}\right)^{1-\alpha_{p}}} & =\frac{1}{2 T_{c}}-\int_{\frac{\delta \tau}{2}}^{\infty} d y f(y) e^{-2 \hat{J}\left(k_{c}\right) y}+\mathcal{O}(\delta)+\frac{K_{p}}{\gamma_{p}-1}\left[\left(z-\frac{1}{t^{\prime}+\frac{\tau}{2}}\right)^{\gamma_{p}-1}\right]_{\frac{2}{\tau}}^{\frac{2}{\delta \tau}} \\
& \sim \frac{1}{2 T_{c}}-K_{p} \int_{\frac{\delta \tau}{2}}^{\infty} \frac{d y}{y^{\gamma_{p}}}+\frac{K_{p}}{\gamma_{p}-1}\left(\frac{2}{\tau}\right)^{\gamma_{p}-1}\left[\frac{1}{\delta^{\gamma_{p}-1}}(1+\mathcal{O}(\delta))^{\gamma_{p}-1}-\left(\frac{2 t^{\prime}}{2 t^{\prime}+\tau}\right)^{\gamma_{p}-1}\right] \\
& \sim \frac{1}{2 T_{c}}+\frac{K_{p}}{1-\gamma_{p}}\left[\frac{4 t^{\prime}}{\tau\left(2 t^{\prime}+\tau\right)}\right]^{\gamma_{p}-1},
\end{aligned}
$$

que, substituído em (A.141), fornece

$$
C\left(t, t^{\prime}\right) \sim \frac{2 K_{p} T_{c} 2^{\gamma_{p}}}{\gamma_{p}-1} t^{1-\gamma_{p}} \frac{x^{1-\frac{\gamma_{p}}{2}}(x-1)^{1-\gamma_{p}}}{x+1}
$$

Quanto à função resposta, de (A.132) e (A.69), vem

$$
\begin{aligned}
R\left(t, t^{\prime}\right) & \sim f\left(\frac{\tau}{2}\right) \sqrt{\frac{e^{2 \hat{J}\left(k_{c}\right) t^{\prime} / t^{\prime-\alpha_{p}}}}{e^{2 \hat{J}\left(k_{c}\right) t} / t^{1-\alpha_{p}}}} \\
& =f\left(\frac{\tau}{2}\right) e^{-\hat{J}\left(k_{c}\right) \tau} x^{\frac{1-\alpha_{p}}{2}} \\
& \sim 2^{\gamma_{p}} K_{p} t^{\prime-\gamma_{p}}(x-1)^{-\gamma_{p}} x^{\frac{1-\alpha_{p}}{2}}
\end{aligned}
$$

e o coeficiente de flutuação - dissipação é, de (A.144) e (A.145),

$$
X\left(t, t^{\prime}\right) \sim \frac{\left(\gamma_{p}-1\right)(x+1)^{2}}{\left(\gamma_{p} x+\gamma_{p}-2\right)(x+1)-2(x-1)}
$$


A.11.2.2- $d=\bar{d}$

Para $d=\bar{d}$, tem-se $\psi(t) \sim \frac{A_{1}}{2 T_{c} F_{p}} \frac{e^{2 j\left(k_{c}\right) t}}{\ln t}$ (vide (A.110)) e o regime estacionário, $1 \sim \tau \ll t^{\prime}$, prevê, de (A.130),

$$
\begin{aligned}
C\left(t, t^{\prime}\right) & \sim \frac{\sqrt{\ln \left(t^{\prime}+\tau\right) \ln t^{\prime}}}{e^{\hat{J}\left(k_{c}\right)\left(2 t^{\prime}+\tau\right)}}\left[\frac{e^{\hat{J}\left(k_{c}\right)\left(2 t^{\prime}+\tau\right)}}{\ln \left(t^{\prime}+\frac{\tau}{2}\right)}-2 T_{c} \int_{0}^{\frac{\tau}{2}} d y f(y) \frac{e^{\hat{J}\left(k_{c}\right)\left(2 t^{\prime}+\tau-2 y\right)}}{\ln \left(t^{\prime}+\frac{\tau}{2}-y\right)}\right] \\
& \sim C_{e q, c}(\tau)
\end{aligned}
$$

e, de (A.132),

$$
\begin{aligned}
R\left(t, t^{\prime}\right) & \sim f\left(\frac{\tau}{2}\right) \sqrt{\frac{e^{2 \hat{J}\left(k_{c}\right) t^{\prime}} / \ln t^{\prime}}{e^{2 \hat{J}\left(k_{c}\right) t / \ln \left(t^{\prime}+\tau\right)}}} \\
& =f\left(\frac{\tau}{2}\right) \frac{e^{-\hat{J}\left(k_{c}\right) \tau}}{\sqrt{1+\frac{\ln \left(1+\tau / t^{\prime}\right)}{\ln t^{\prime}}}} \\
& \sim f\left(\frac{\tau}{2}\right) e^{-\hat{J}\left(k_{c}\right) \tau}
\end{aligned}
$$

assegurando, novamente, $X\left(t, t^{\prime}\right) \sim 1$ no regime estacionário.

No regime de "aging", $1 \ll \tau \sim t^{\prime}$, e a partir de (A.130), (A.113), tem-se

$$
\begin{aligned}
C\left(t, t^{\prime}\right) & \sim \frac{\sqrt{\ln \left(t^{\prime}+\tau\right) \ln t^{\prime}}}{e^{\hat{J}\left(k_{c}\right)\left(2 t^{\prime}+\tau\right)}}\left[\frac{e^{\hat{J}\left(k_{c}\right)\left(2 t^{\prime}+\tau\right)}}{\ln \left(t^{\prime}+\frac{\tau}{2}\right)}-2 T_{c} \int_{0}^{\frac{\tau}{2}} d y f(y) \frac{e^{\hat{J}\left(k_{c}\right)\left(2 t^{\prime}+\tau-2 y\right)}}{\ln \left(t^{\prime}+\frac{\tau}{2}-y\right)}\right] \\
& =\frac{\sqrt{\ln \left(t^{\prime}+\tau\right) \ln t^{\prime}}}{\ln \left(t^{\prime}+\frac{\tau}{2}\right)}\left[1-2 T_{c} \int_{0}^{\frac{\tau}{2}} \frac{d y f(y) e^{-2 \hat{J}\left(k_{c}\right) y}}{\left.1+\frac{\ln \left[1-y /\left(t^{\prime}+\frac{\tau}{2}\right)\right]}{\ln \left(t^{\prime}+\frac{\tau}{2}\right)}\right]}\right.
\end{aligned}
$$

e escolhendo um $\delta>0$ tal que $1 / t^{\prime} \ll \delta \ll 1^{15}$, a integral em (A.149) pode ser desenvolvida como

$$
\begin{aligned}
\int_{0}^{\frac{\tau}{2}} \frac{d y f(y) e^{-2 \hat{J}\left(k_{c}\right) y}}{1+\frac{\ln \left[1-y /\left(t^{\prime}+\frac{\tau}{2}\right)\right]}{\ln \left(t^{\prime}+\frac{\tau}{2}\right)}} & =\int_{0}^{\frac{\tau}{2}} d y f(y) e^{-2 \hat{J}\left(k_{c}\right) y}\left[1-\frac{\ln \left(1-y /\left(t^{\prime}+\frac{\tau}{2}\right)\right)}{\ln \left(t^{\prime}+\frac{\tau}{2}\right)}+\mathcal{O}\left(\ln ^{-2}\left(t^{\prime}+\frac{\tau}{2}\right)\right)\right] \\
& \sim \int_{0}^{\frac{\tau}{2}} d y f(y) e^{-2 \hat{J}\left(k_{c}\right) y}-\frac{1}{\ln \left(t^{\prime}+\frac{\tau}{2}\right)} \int_{0}^{\frac{\tau}{2}} d y f(y) e^{-2 \hat{J}\left(k_{c}\right) y} \ln \left(1-\frac{y}{t^{\prime}+\frac{\tau}{2}}\right) \\
& =\frac{1}{2 T_{c}}+\frac{K_{p}}{1-\gamma_{p}}\left(\frac{\tau}{2}\right)^{1-\gamma_{p}}-\frac{1}{\ln \left(t^{\prime}+\frac{\tau}{2}\right)} \int_{0}^{\frac{\tau}{2}} d y f(y) e^{-2 \hat{J}\left(k_{c}\right) y} \ln \left(1-\frac{y}{t^{\prime}+\frac{\tau}{2}}\right) .
\end{aligned}
$$

${ }^{15}$ Novamente, $\delta=1 / \sqrt{t^{\prime}}$ é uma escolha possível 
onde (A.69) e a relação auxiliar (i) (seção A.10) foi novamente usada. Resta agora demonstrar que o terceiro termo é desprezível frente ao segundo para $1 \ll \tau \sim t^{\prime}$. Escolhendo $\eta>0$ tal que $1 / \tau \ll \eta \ll$ $\tau^{1-\gamma_{p}} \ln \tau, \operatorname{vem}^{16}$, de (A.69) e da relação auxiliar (i) (seção A.10),

$$
\begin{aligned}
\left|\int_{0}^{\frac{\tau}{2}} d y f(y) e^{-2 \hat{J}\left(k_{c}\right) y} \ln \left(1-\frac{y}{t^{\prime}+\frac{\tau}{2}}\right)\right| \leq & \frac{1}{t^{\prime}+\frac{\tau}{2}} \int_{0}^{\frac{\eta \tau}{2}} d y f(y) e^{-2 \hat{J}\left(k_{c}\right) y} \mathcal{O}(\eta \tau)+ \\
& +K_{p} \int_{\frac{\eta \tau}{2} \frac{d y}{y^{\gamma_{p}}}\left|\ln \left(1-\frac{y}{t^{\prime}+\frac{\tau}{2}}\right)\right|}^{\leq^{\frac{\tau}{2}}} \\
& \mathcal{O}(\eta) \underbrace{\left[\frac{1}{2 T_{c}}-\int_{\frac{\eta \tau}{2}}^{\infty} d y f(y) e^{-2 \hat{J}\left(k_{c}\right) y}\right]}_{\mathcal{O}(1)}+\mathcal{O}\left(\frac{\tau^{2-\gamma_{p}}}{t^{\prime}+\frac{\tau}{2}}\right) \\
= & \mathcal{O}(\eta) .
\end{aligned}
$$

Logo, o terceiro termo é $\mathcal{O}\left(\frac{\eta}{\ln \left(t^{\prime}+\frac{\tau}{2}\right)}\right)$, que é desprezível (para $1 \ll \tau \sim t^{\prime}$ ) frente ao segundo termo. Reunindo as informações acima, chega-se finalmente a

$$
C\left(t, t^{\prime}\right) \sim \frac{T_{c} K_{p} 2^{\gamma_{p}}}{\gamma_{p}-1} t^{1-\gamma_{p}}(x-1)^{1-\gamma_{p}}\left[1+\frac{\ln (x+1)}{\ln t^{\prime}}\right]^{-1} \sqrt{1+\frac{\ln x}{\ln t^{\prime}}},
$$

enquanto que o cálculo da função resposta é mais simples, sendo

$$
\begin{aligned}
R\left(t, t^{\prime}\right) & \sim f\left(\frac{\tau}{2}\right) e^{-\hat{J}\left(k_{c}\right) \tau} \sqrt{1+\frac{\ln x}{\ln t^{\prime}}} \\
& \sim K_{p} 2^{\gamma_{p}} t^{\prime^{-\gamma_{p}}}(\dot{x}-1)^{-\gamma_{p}} \sqrt{1+\frac{\ln x}{\ln t^{\prime}}}
\end{aligned}
$$

O coeficiente de flutuação - dissipação, então, é

$$
X\left(t, t^{\prime}\right) \sim \frac{2\left(\gamma_{p}-1\right)}{\frac{x-1+2\left(\gamma_{p}-1\right) \ln t^{\prime}}{\ln t^{\prime}+\ln (x+1)}-\left(\frac{x-1}{x+1}\right) \frac{\ln t^{\prime}}{\left[\ln t^{\prime}+\ln (x+1)\right]^{2}}}
$$

\section{A.11.2.3- $d>\bar{d}$}

Finalmente, para $d>\bar{d}$, a autocorrelação é, de (A.130), (A.113) e da relação auxiliar (i) (seção A.10),

$$
C\left(t, t^{\prime}\right) \sim 1-2 T_{c} \int_{0}^{\frac{\tau}{2}} d y f(y) e^{-2 \hat{J}\left(k_{c}\right) y}=C_{e q, c}(\tau) .
$$

\footnotetext{
${ }^{16} \hat{E}$ suficiente tomar, por exemplo, $\eta=\sqrt{\tau^{-\gamma_{p}} \ln \tau}$.
} 
A função resposta é obtida de (A.113) e (A.132), sendo

$$
R\left(t, t^{\prime}\right) \sim f\left(\frac{\tau}{2}\right) e^{-\hat{J}\left(k_{c}\right) \tau}
$$

No regime estacionário (onde $\tau \sim 1$ ), as expansões assintóticas das funções de dois tempos são descritas por (A.155) e (A.156); neste caso, tem-se ainda $X\left(t, t^{\prime}\right) \sim 1$. Por outro lado, no regime de "aging", quando então $\tau \gg 1$, essas expressões têm os seguintes comportamentos assintóticos:

$$
C\left(t, t^{\prime}\right) \sim \frac{2^{\gamma_{p}} K_{p} T_{c}}{\gamma_{p}-1} \tau^{1-\gamma_{p}}=\frac{2^{\gamma_{p}} K_{p} T_{c}}{\gamma_{p}-1} t^{1-\gamma_{p}}(x-1)^{1-\gamma_{p}}
$$

e

$$
R\left(t, t^{\prime}\right) \sim 2^{\gamma_{p}} K_{p} \tau^{-\gamma_{p}}=2^{\gamma_{p}} K_{p} t^{\prime^{-\gamma_{p}}}(x-1)^{-\gamma_{p}}
$$

Aqui, novamente o teorema flutuação - dissipação é respeitado com $X\left(t, t^{\prime}\right) \sim 1$.

\section{A.11.3- Dinâmica subcrítica}

Similarmente à dinâmica crítica, as duas escalas de tempo presentes naquela estão também aqui.

No regime de tempo estacionário, $1 \sim \tau \ll t^{\prime}$, de (A.130), usando (A.117) e (A.69),

$$
\begin{aligned}
C\left(t, t^{\prime}\right) & \sim \frac{M_{e q}^{4}\left[t^{\prime}\left(t^{\prime}+\tau\right)\right]^{\frac{\gamma}{2}}}{K_{p} e^{\hat{J}\left(k_{c}\right)\left(2 t^{\prime}+\tau\right)}}\left\{\frac{1}{M_{e q}^{4}} \frac{K_{p} e^{\hat{\jmath}\left(k_{c}\right)\left(2 t^{\prime}+\tau\right)}}{\left(t^{\prime}+\frac{\tau}{2}\right)^{\gamma}}-2 T \int_{0}^{\frac{\tau}{2}} d y f(y) \frac{1}{M_{e q}^{4}} \frac{K_{p} e^{\hat{J}\left(k_{c}\right)\left(2 t^{\prime}+\tau-2 y\right)}}{\left(t^{\prime}+\frac{\tau}{2}-y\right)^{\gamma}}\right\} \\
& =\left[\frac{4 t^{\prime}\left(t^{\prime}+\tau\right)}{\left(2 t^{\prime}+\tau\right)^{2}}\right]^{\frac{\gamma}{2}}-2 T\left[t^{\prime}\left(t^{\prime}+\tau\right)\right]^{\frac{\gamma}{2}} \int_{0}^{\frac{\tau}{2}} d y \frac{f(y) e^{-2 \hat{J}\left(k_{c}\right) y}}{\left(t^{\prime}+\frac{\tau}{2}-y\right)^{\gamma}} \\
& =1-2 T \int_{0}^{\frac{\tau}{2}} d y f(y) e^{-2 \hat{J}\left(k_{c}\right) y}+\mathcal{O}\left(\frac{\tau}{t^{\prime}}\right) \\
& \sim 1-2 T \int_{0}^{\infty} d y f(y) e^{-2 \hat{J}\left(k_{c}\right) y}+2 T \int_{\frac{\tau}{2}}^{\infty} d y f(y) e^{-2 \hat{J}\left(k_{c}\right) y} \\
& =1-\frac{T}{T_{c}}+T \int_{\tau}^{\infty} d y f\left(\frac{y}{2}\right) e^{-\hat{J}\left(k_{c}\right) y}
\end{aligned}
$$

onde, na última passagem foi usada a equação auxiliar (i) (seção A.10). Com a definição de $M_{e q}^{2}$, dada em (A.115), vem

$$
C\left(\tau, t^{\prime}\right) \sim M_{e q}^{2}+\left(1-M_{e q}^{2}\right) C_{e q, c}(\tau)
$$


Tomando, agora, $\tau \gg 1$ (mas com $\tau \ll t^{\prime}$ ), tem-se, de (A.69),

$$
C_{e q, c}(\tau \gg 1) \sim \int_{\tau}^{\infty} d y \frac{K_{p} e^{\hat{J}\left(k_{c}\right) y}}{\left(\frac{y}{2}\right)^{\gamma_{p}}} e^{-\hat{J}\left(k_{c}\right) y}=\frac{K_{p} 2^{\gamma_{p}}}{\gamma_{p}-1} \tau^{1-\gamma_{p}}
$$

Usando (A.117), a função resposta, descrita por

$$
R\left(t, t^{\prime}\right) \sim f\left(\frac{\tau}{2}\right) \sqrt{\frac{f\left(t^{\prime}\right)}{f(t)}}
$$

nos dois regimes de tempo, assume a forma

$$
R\left(t, t^{\prime}\right) \sim f\left(\frac{\tau}{2}\right) e^{-\hat{J}\left(k_{c}\right) \tau}\left(1+\frac{\tau}{t^{\prime}}\right)^{\frac{\gamma_{p}}{2}}=f\left(\frac{\tau}{2}\right) e^{-\hat{J}\left(k_{c}\right) \tau}+\mathcal{O}\left(\frac{\tau}{t^{\prime}}\right)
$$

no caso estacionário; não é difícil ver que nesta escala de tempo o teorema flutuação - dissipação é válido $\operatorname{com} X\left(t, t^{\prime}\right) \sim 1$.

No regime de "aging", $1 \ll \tau \sim t^{\prime}$, a autocorrelação pode ser escrita como

$$
C\left(t, t^{\prime}\right) \sim M_{e q}^{4}\left[\frac{4 x}{(x+1)^{2}}\right]^{\frac{\gamma_{p}}{2}}\left\{1+2 T \int_{0}^{t^{\prime}} \frac{d y e^{-2 \hat{J}\left(k_{c}\right) y} \psi(y)}{\left[1-\frac{2 y}{t^{\prime}(x+1)}\right]^{\gamma_{p}}}\right\}
$$

usando (A.117) e (A.69) em (A.129). Tome, agora, $\delta>0$ tal que $1 / t^{\prime} \ll \delta \ll 1$ para $t^{\prime} \sim \infty^{17}$. Repartindo a integral em (A.164) em dois intervalos complementares, $\left[0, t^{\prime}\right]=\left[0, \delta t^{\prime}\right) \cup\left[\delta t^{\prime}, t^{\prime}\right]$, tem-se, lembrando que $x>1$ e $\gamma_{p} \in(1,2)$ para $d_{c}<d<\bar{d}$,

$$
\begin{aligned}
\int_{0}^{\delta t^{\prime}} \frac{d y e^{-2 \hat{J}\left(k_{c}\right) y} \psi(y)}{\left[1-\frac{2 y}{t^{\prime}(x+1)}\right]^{\gamma_{p}}+\int_{\delta t^{\prime}}^{t^{\prime}} \frac{d y e^{-2 \hat{J}\left(k_{c}\right) y} \psi(y)}{\left[1-\frac{2 y}{t^{\prime}(x+1)}\right]^{\gamma_{p}}}} & \sim \int_{0}^{\delta t^{\prime}} d y e^{-2 \hat{J}\left(k_{c}\right) y} \psi(y)[1+\mathcal{O}(\delta)]+\int_{\delta t^{\prime}} \frac{d y e^{-2 \hat{J}\left(k_{c}\right) y} f(y)}{M_{e q}^{4}\left[1-\frac{2 y}{t^{\prime}(x+1)}\right]^{\gamma_{p}}} \\
& \sim \int_{0}^{\delta t^{\prime}} d y e^{-2 \hat{J}\left(k_{c}\right) y} \psi(y)+\frac{K_{p}}{M_{e q}^{4}} \int_{\delta t^{\prime}}^{t^{\prime}} \frac{d y}{y^{\gamma_{p}}\left[1-\frac{2 y}{t^{\prime}(x+1)}\right]^{\gamma_{p}}} \\
& \sim \int_{0}^{\delta t^{\prime}} d y e^{-2 \hat{J}\left(k_{c}\right) y} \psi(y)+\frac{K_{p}}{M_{e q}^{4}} \int_{\delta t^{\prime}}^{t^{\prime}} \frac{d y}{y^{\gamma_{p}}}\left[1+\mathcal{O}\left(\frac{y}{t^{\prime}}\right)\right] \\
& \sim \int_{0}^{\infty} d y e^{-2 \hat{J}\left(k_{c}\right) y} \psi(y)-\int_{\delta t^{\prime}}^{\infty} d y e^{-2 \hat{J}\left(k_{c}\right) y} \psi(y)+\mathcal{O}\left(\left(\delta t^{\prime}\right)^{1-\gamma_{p}}\right) .
\end{aligned}
$$

O segundo termo na última linha de (A.165) é também $\mathcal{O}\left(\left(\delta t^{\prime}\right)^{1-\gamma_{p}}\right)$ (basta usar (A.117) e (A.69)); pela relação auxiliar (iii) (seção A.10),

\footnotetext{
${ }^{17}$ Aqui, é suficiente considerar, por exemplo, $\delta=1 / \sqrt{t^{\prime}}$.
} 


$$
\int_{0}^{t^{\prime}} \frac{d y e^{-2 \hat{J}\left(k_{c}\right) y} \psi(y)}{\left[1-\frac{2 y}{t^{\prime}(x+1)}\right]^{\gamma_{p}}} \sim \frac{1}{2 T_{\mathbf{c}} M_{e q}^{2}},
$$

que é o comportamento assintótico da integral em (A.164). A partir deste resultado, é imediato que a autocorrelação é

$$
C\left(t, t^{\prime}\right) \sim M_{e q}^{2}\left[\frac{4 x}{(x+1)^{2}}\right]^{\frac{\gamma_{p}}{2}} .
$$

O cálculo assintótico da função resposta é mais simples, fornecendo

$$
R\left(t, t^{\prime}\right) \sim \frac{K_{p} e^{\hat{j}\left(k_{c}\right) \tau}}{\left(\frac{\tau}{2}\right)^{\gamma_{p}}} e^{-\hat{J}\left(k_{c}\right) \tau} x^{\frac{\gamma_{p}}{2}}=K_{p} 2^{\gamma_{p}} t^{\prime^{-\gamma_{p}}} x^{\frac{\gamma_{p}}{2}}(x-1)^{-\gamma_{p}}
$$

O coeficiente de flutuação - dissipação assintótico é, por (A.167) e (A.168),

$$
X\left(t, t^{\prime}\right) \sim \frac{2 T K_{p}}{\gamma_{p} M_{e q}^{2}} t^{\prime 1-\gamma_{p}}\left(\frac{x+1}{x-1}\right)^{1+\gamma_{p}}
$$




\section{Apêndice B}

\section{B.1- Energia livre variacional}

Para uma função de Hamilton

$$
H\left(\left\{\sigma_{x}\right\}\right)=-\sum_{\substack{x, x^{\prime} \in \Lambda_{N} \\ x \neq x^{\prime}}} J_{x, x^{\prime}} \sigma_{x} \sigma_{x^{\prime}}-\sum_{x \in \Lambda_{N}} H_{x} \sigma_{x}
$$

onde $\sigma_{x} \in\{-1,1\}$ são spins de Ising e $\left\{H_{x}\right\}$ e $\left\{J_{x, x^{\prime}}\right\}$ constituem conjuntos de variáveis aleatórias, idênticas e independentemente distribuídas, com distribuições dadas, respectivamente, por

$$
p_{H}\left(H_{x}\right)=\frac{1}{2} \delta\left(H_{x}-H_{R}\right)+\frac{1}{2} \delta\left(H_{x}+H_{R}\right)
$$

e

$$
p_{J}\left(J_{x, x^{\prime}}\right)=\left(1-\frac{c}{N}\right) \delta\left(J_{x, x^{\prime}}\right)+\frac{c}{N} \rho\left(J_{x, x^{\prime}}\right)
$$

onde $\rho$ também é uma função distribuição de probabilidades,

$$
\rho\left(J_{x, x^{\prime}}\right)=\delta\left(J_{x, x^{\prime}}-J_{0}\right)
$$

calcula-se a energia livre pelo método das réplicas. Considere, inicialmente, a função de partição

$$
Z\left(\beta,\left\{J_{x, x^{\prime}}\right\},\left\{H_{x}\right\}\right)=\operatorname{Tr}_{\left\{\sigma_{x}\right\}} e^{-\beta H\left(\beta,\left\{H_{x}\right\},\left\{J_{x, x^{\prime}}\right\}\right)},
$$

onde o traço representa a soma sobre todas as $2^{N}$ configurações dos $N$ spins localizados numa rede finita $\Lambda_{N}$. Para aplicar o truque de réplicas, é necessário calcular, inicialmente, a esperança (em relação a $p_{H}$ e $\left.p_{J}\right)$ de $n$ cópias da função de partição, $Z^{n}\left(\beta,\left\{J_{x, x^{\prime}}\right\},\left\{H_{x}\right\}\right)$ : 


$$
\begin{aligned}
\mathbb{E}_{H}\left\{\mathbb { E } _ { J } \left\{Z ^ { n } \left(\beta,\left\{J_{\left.\left.\left.\left.x, x^{\prime}\right\},\left\{H_{x}\right\}\right)\right\}\right\}=}\right.\right.\right.\right. & \mathbb{E}_{H}\left\{\mathbb{E}_{J}\left\{\operatorname{Tr}_{\left\{\sigma_{x \alpha}\right\}} \exp \left[\beta \sum_{\substack{x, x^{\prime} \in \Lambda_{N} \\
x \neq x^{\prime}}} J_{x, x^{\prime}} \sum_{\alpha=1}^{n} \sigma_{x \alpha} \sigma_{x^{\prime} \alpha}+\beta \sum_{x \in \Lambda_{N}} H_{x} \sum_{\alpha=1}^{n} \sigma_{x \alpha}\right]\right\}\right\} \\
= & \left.\left.\operatorname{Tr}_{\left\{\sigma_{x \alpha}\right\}}\left[\prod_{\substack{x, x^{\prime} \in \Lambda_{N} \\
x \neq x^{\prime}}} \int_{\mathbb{R}} d J_{x, x^{\prime}} e^{\beta J_{x, x^{\prime}} \sum_{\alpha=1}^{n} \sigma_{x \alpha} \sigma_{x^{\prime} \alpha}}\right]\left[\prod_{x \in \Lambda_{N}} \int_{\mathbb{R}} d H_{x} e^{\beta H_{x} \sum_{\alpha=1}^{n} \sigma_{x \alpha}}\right]\right)\right] \\
= & \operatorname{Tr}_{\left\{\sigma_{x \alpha}\right\}} \exp \left\{\sum _ { \substack { x , x ^ { \prime } \in \Lambda _ { N } \\
x \neq x ^ { \prime } } } \operatorname { l n } \left[1+\frac{c}{N}\left(\int_{\mathbb{R}} d J_{x, x^{\prime}} \rho\left(J_{x, x^{\prime}}\right) e^{\left.\beta J_{x, x^{\prime}} \sum_{\alpha=1}^{n} \sigma_{x \alpha} \sigma_{x^{\prime} \alpha}-1\right)}\right.\right.\right. \\
& \left.+\sum_{x \in \Lambda_{N}} \ln \int_{\mathbb{R}} d H_{x} p_{H}\left(H_{x}\right) e^{\beta H_{x} \sum_{\alpha=1}^{n} \sigma_{x \alpha}}\right\} \\
= & \operatorname{Tr}_{\left\{\sigma_{x \alpha}\right\}} \exp \left\{-\frac{N c}{2}+\frac{c}{N} \sum_{\substack{x, x^{\prime} \in \Lambda_{N} \\
x \neq x^{\prime}}} \int_{\mathbb{R}} d J_{x, x^{\prime}} \rho\left(J_{x, x^{\prime}}\right) e^{\beta J_{x, x^{\prime}} \sum_{\alpha=1}^{n} \sigma_{x \alpha} \sigma_{x^{\prime} \alpha}+}\right. \\
& \left.+\sum_{x \in \Lambda_{N}} \ln \int_{\mathbb{R}} d H_{x} p_{H}\left(H_{x}\right) e^{\beta H_{x} \sum_{\alpha=1}^{n} \sigma_{x \alpha}}+\mathcal{O}(1)\right\}
\end{aligned}
$$

e agora o traço é tomado sobre $2^{n N}$ configurações dos $n N$ spins.

Substituindo, agora,

$$
\begin{aligned}
e^{\beta J_{x, x^{\prime}} \sum_{\alpha=1}^{n} \sigma_{x \alpha} \sigma_{x^{\prime} \alpha}} & =\prod_{\alpha}^{n} e^{\beta J_{x, x^{\prime}} \sigma_{x \alpha} \sigma_{x^{\prime} \alpha}}=\prod_{\alpha}^{n}\left[\sum_{k=0}^{\infty} \frac{\left(\beta J_{x, x^{\prime}}\right)^{2 k}}{(2 k) !}+\sigma_{x \alpha} \sigma_{x^{\prime} \alpha} \sum_{k=0}^{\infty} \frac{\left(\beta J_{x, x^{\prime}}\right)^{2 k+1}}{(2 k+1) !}\right] \\
& =\cosh ^{n}\left(\beta J_{x, x^{\prime}}\right) \prod_{\alpha=1}^{n}\left[1+\sigma_{x \alpha} \sigma_{x^{\prime} \alpha} \tanh \left(\beta J_{x, x^{\prime}}\right)\right]
\end{aligned}
$$

e

$$
\int_{\mathbb{R}} d J_{x, x^{\prime}} \rho\left(J_{x, x^{\prime}}\right) e^{\beta J_{x, x^{\prime}} \sum_{\alpha=1}^{n} \sigma_{x \alpha} \sigma_{x^{\prime} \alpha}}=\sum_{r=0}^{n} b_{r} \sum_{\left(\alpha_{1}, \cdots, \alpha_{r}\right)}\left(\sigma_{x \alpha_{1}} \cdots \sigma_{x \alpha_{r}}\right)\left(\sigma_{x^{\prime} \alpha_{1}} \cdots \sigma_{x^{\prime} \alpha_{r}}\right),
$$

onde $\sum_{\left(\alpha_{1}, \cdots, \alpha_{r}\right)}$ indica a soma nos índices $1 \leq \alpha_{1}<\cdots<\alpha_{r} \leq n$, em (B.6), chega-se a

$$
\begin{aligned}
\mathbb{E}_{H}\left\{\mathbb{E}_{J}\left\{Z^{n}\left(\beta,\left\{J_{x, x^{\prime}}\right\},\left\{H_{x}\right\}\right)\right\}\right\}= \\
=\operatorname{Tr}_{\left\{\sigma_{x \alpha}\right\}} \exp \left[-\frac{c N}{2}+\frac{c}{N} \sum_{r=0}^{n} b_{r} \sum_{\left(\alpha_{1}, \cdots, \alpha_{r}\right)}\left(\sigma_{x \alpha_{1}} \cdots \sigma_{x \alpha_{r}}\right)\left(\sigma_{x^{\prime} \alpha_{1}} \cdots \sigma_{x^{\prime} \alpha_{r}}\right)+\right. \\
\left.\quad+\sum_{x \in \Lambda_{N}} \ln \int_{\mathbb{R}} d H_{x} p_{H}\left(H_{x}\right) e^{\beta H_{x} \sum_{\alpha=1}^{n} \sigma_{x \alpha}}+\mathcal{O}(1)\right]
\end{aligned}
$$


A igualdade (B.8) é verificada a posteriori pela determinação dos coeficientes $\left\{b_{r}\right\}$. Considere a equação (B.8) para spin de um único sítio,

$$
\sum_{r=0}^{n} \sum_{\left(\alpha_{1}, \cdots, \alpha_{r}\right)} b_{r} \sigma_{\alpha_{1}} \cdots \sigma_{\alpha_{r}}=\int_{\mathbb{R}} d J \rho(J) e^{\beta J \sum_{\alpha=1}^{n} \sigma_{\alpha}}
$$

Multiplicando ambos membros por $\sigma_{\gamma_{1}} \cdots \sigma_{\gamma_{s}}$ e tomando o traço nas $2^{n}$ configurações, vem

$$
\begin{aligned}
2^{n} b_{s} & =\int_{\mathbb{R}} d J \rho(J) \operatorname{Tr}_{\left\{\sigma_{\alpha}\right\}} \sigma_{\gamma_{1}} \cdots \sigma_{\gamma_{s}} e^{\beta J \sum_{\alpha=1}^{n} \sigma_{\alpha}} \\
& =\int_{\mathbb{R}} d J \rho(J) \operatorname{Tr}_{\left\{\sigma_{\alpha}\right\}} 2^{s} \sinh ^{s}(\beta J) 2^{n-s} \cosh ^{n-s}(\beta J),
\end{aligned}
$$

ou

$$
b_{r}=\int_{\mathbb{R}} d J \rho(J) \cosh ^{n}(\beta J) \tanh ^{r}(\beta J)
$$

Os conjuntos $\left\{H_{x}\right\}$ e $\left\{J_{x, x^{\prime}}\right\}$ representam variáveis aleatórias independentes e identicamente distribuídas: não há uma dependência espacial, e os índices referentes às posições na rede podem ser omitidos. Usando, agora,

$$
\sum_{\substack{x, x^{\prime} \in \Lambda_{N} \\ x \neq x^{\prime}}}\left(\sigma_{x \alpha_{1}} \cdots \sigma_{x \alpha_{r}}\right)\left(\sigma_{x^{\prime} \alpha_{1}} \cdots \sigma_{x^{\prime} \alpha_{r}}\right)=\frac{1}{2}\left[\left(\sum_{x \in \Lambda_{N}} \sigma_{x \alpha_{1}} \cdots \sigma_{x \alpha_{r}}\right)^{2}-N\right],
$$

vem

$$
\begin{aligned}
\mathbb{E}_{H}\left\{\mathbb{E}_{J}\left\{Z^{n}\left(\beta,\left\{J_{x, x^{\prime}}\right\},\left\{H_{x}\right\}\right)\right\}\right\}= \\
=\operatorname{Tr}_{\left\{\sigma_{x}^{\alpha}\right\}} \exp \left[-\frac{c N}{2}+\frac{c}{2 N} \sum_{r=0}^{n} b_{r} \sum_{\left(\alpha_{1}, \cdots, \alpha_{r}\right)}\left(\sum_{x \in \Lambda_{N}} \sigma_{x \alpha_{1}} \cdots \sigma_{x \alpha_{r}}\right)^{2}+\right. \\
\left.\quad+\sum_{x \in \Lambda_{N}} \ln \int_{\mathbb{R}} d H p_{H}(H) e^{\beta H \sum_{\alpha=1}^{n} \sigma_{x \alpha}}+\mathcal{O}(1)\right]
\end{aligned}
$$

Da identidade de Gauss,

$$
e^{\lambda a^{2}}=\frac{1}{\sqrt{\pi}} \int_{\mathbb{R}} d x e^{-x^{2}+2 \sqrt{\lambda} a x}
$$

onde $a \in \mathbb{R}$ e $\lambda>0$, tem-se 


$$
\begin{aligned}
\exp & {\left[\frac{c}{2 N} \sum_{r=0}^{n} b_{r} \sum_{\left(\alpha_{1}, \cdots, \alpha_{r}\right)}\left(\sum_{x \in \Lambda_{N}} \sigma_{x \alpha_{1}} \cdots \sigma_{x \alpha_{r}}\right)^{2}\right]=} \\
= & \int \exp \left\{-\sum_{r=0}^{n} \sum_{\left(\alpha_{1}, \cdots, \alpha_{r}\right)}\left[x_{\alpha_{1} \cdots \alpha_{r}}^{2}-2 x_{\alpha_{1} \cdots \alpha_{r}} \sqrt{\frac{c b_{r}}{2 N}} \sum_{x \in \Lambda_{N}} \sigma_{x \alpha_{1}} \cdots \sigma_{x \alpha_{r}}\right]\right\} \prod_{r=0}^{n} \prod_{\left(\alpha_{1}, \cdots, \alpha_{r}\right)} \frac{d x_{\alpha_{1} \cdots \alpha_{r}}}{\sqrt{\pi}} \\
= & \int \exp \left[-\frac{N c}{2} \sum_{r=0}^{n} \sum_{\left(\alpha_{1}, \cdots, \alpha_{r}\right)} b_{r} q_{\alpha_{1} \cdots \alpha_{r}}^{2}+c \sum_{r=0}^{n} \sum_{\left(\alpha_{1}, \cdots, \alpha_{r}\right)} b_{r} q_{\alpha_{1} \cdots \alpha_{r}} \sum_{x \in \Lambda_{N}} \sigma_{x \alpha_{1}} \cdots \sigma_{x \alpha_{r}}\right] \times \\
& \times \prod_{r=0}^{n} \prod_{\left(\alpha_{1}, \cdots, \alpha_{r}\right)} \sqrt{\frac{c N b_{r}}{2 \pi}} d q_{\alpha_{1} \cdots \alpha_{r}},
\end{aligned}
$$

onde, na última passagem, foi introduzida a mudança de variável $x_{\alpha_{1} \cdots \alpha_{r}}=\sqrt{\frac{N c b_{x}}{2}} q_{\alpha_{1} \cdots \alpha_{r}}$. A combinação de (B.14) e (B.16) fornece

$$
\begin{aligned}
& \mathbb{E}_{H}\left\{\mathbb{E}_{J}\left\{Z^{n}\left(\beta,\left\{J_{x, x^{\prime}}\right\},\left\{H_{x}\right\}\right)\right\}\right\}= \\
& =\operatorname{Tr}_{\left\{\sigma_{x \alpha}\right\}} \int \exp \left[-\frac{N c}{2}-\frac{N c}{2} \sum_{r=0}^{n} \sum_{\left(\alpha_{1}, \cdots, \alpha_{r}\right)} b_{r} q_{\alpha_{1} \cdots \alpha_{r}}^{2}+c \sum_{r=0}^{n} \sum_{\left(\alpha_{1}, \cdots, \alpha_{r}\right)} b_{r} q_{\alpha_{1} \cdots \alpha_{r}} \sum_{x \in \Lambda_{N}} \sigma_{x \alpha_{1}} \cdots \sigma_{x \alpha_{r}}+\right. \\
& \left.+\sum_{x \in \Lambda_{N}} \ln \int_{\mathbb{R}} d H p_{H}(H) e^{\beta H \sum_{\alpha=1}^{n} \sigma_{x \alpha}}+\mathcal{O}(1)\right] \prod_{r=0}^{n} \prod_{\left(\alpha_{1}, \cdots, \alpha_{r}\right)} \sqrt{\frac{c N b_{r}}{2 \pi}} d q_{\alpha_{1} \cdots \alpha_{r}} .
\end{aligned}
$$

A identidade e independência das variáveis aleatórias permitem suprimir a dependência dos spins com as suas posições; a expressão acima assume, então, a forma

$$
\begin{aligned}
& \mathbb{E}_{H}\left\{\mathbb{E}_{J}\left\{Z^{n}\left(\beta,\left\{J_{x, x^{\prime}}\right\},\left\{H_{x}\right\}\right)\right\}\right\}= \\
& =\int \exp \left\{-\frac{N c}{2}-\frac{N c}{2} \sum_{r=0}^{n} \sum_{\left(\alpha_{1}, \cdots, \alpha_{r}\right)} b_{r} q_{\alpha_{1} \cdots \alpha_{r}}^{2}+N \ln \operatorname{Tr}_{\left\{\sigma_{\alpha}\right\}} \exp \left[c \sum_{r=0}^{n} \sum_{\left(\alpha_{1}, \cdots, \alpha_{r}\right)} b_{r} q_{\alpha_{1} \cdots \alpha_{r}} \sigma_{\alpha_{1}} \cdots \sigma_{\alpha_{r}}+\right.\right. \\
& \left.\left.\quad+\ln \int_{\mathbb{R}} d H p_{H}(H) e^{\beta H} \sum_{\alpha=1}^{n} \sigma_{\alpha}\right]+\mathcal{O}(1)\right\} \prod_{r=0}^{n} \prod_{\left(\alpha_{1}, \cdots, \alpha_{r}\right)} \sqrt{\frac{c N b_{r}}{2 \pi}} d q_{\alpha_{1} \cdots \alpha_{r}} ;
\end{aligned}
$$

agora, o traço é tomado sobre $2^{n}$ configurações de spins.

A comutação dos limites

$$
\begin{aligned}
f(\beta) & =-\lim _{N \rightarrow \infty} \lim _{n \rightarrow 0} \frac{1}{\beta n N} \ln \mathbb{E}_{H}\left\{\mathbb{E}_{J}\left\{Z^{n}\left(\beta,\left\{H_{x}\right\},\left\{J_{x, x^{\prime}}\right\}\right)\right\}\right\} \\
& =-\lim _{n \rightarrow 0} \lim _{N \rightarrow \infty} \frac{1}{\beta n N} \ln \mathbb{E}_{H}\left\{\mathbb{E}_{J}\left\{Z^{n}\left(\beta,\left\{H_{x}\right\},\left\{J_{x, x^{\prime}}\right\}\right)\right\}\right\}
\end{aligned}
$$

permite o recurso ao método do ponto de sela para se chegar à energia livre estacionária 


$$
\begin{aligned}
f(\beta) & =-\lim _{n \rightarrow 0} \lim _{N \rightarrow \infty} \frac{1}{\beta n N} \ln \exp \left[-N \psi\left(\left\{q_{\alpha_{1} \cdots \alpha_{r}}\right\}\right)\right] \\
& =\lim _{n \rightarrow 0} \frac{1}{\beta n} \inf _{\left\{q_{\alpha_{1} \cdots \alpha_{r}}\right\}} \psi\left(\left\{q_{\alpha_{1} \cdots \alpha_{r}}\right\}\right)
\end{aligned}
$$

onde

$$
\begin{aligned}
\psi\left(\left\{q_{\alpha_{1} \cdots \alpha_{r}}\right\}\right):= & \frac{c}{2}+\frac{c}{2} \sum_{r=0}^{n} \sum_{\left(\alpha_{1}, \cdots, \alpha_{r}\right)} b_{r} q_{\alpha_{1} \cdots \alpha_{r}}^{2}-\ln \operatorname{Tr}_{\left\{\sigma_{\alpha}\right\}} \exp \left[c \sum_{r=0}^{n} \sum_{\left(\alpha_{1}, \cdots, \alpha_{r}\right)} b_{r} q_{\alpha_{1} \cdots \alpha_{r}} \sigma_{\alpha_{1}} \cdots \sigma_{\alpha_{r}}+\right. \\
& \left.+\ln \int_{\mathbb{R}} d H p_{H}(H) e^{\beta H \sum_{\alpha=1}^{n} \sigma_{\alpha}}\right]
\end{aligned}
$$

A extremização de $\psi$,

$$
\frac{\partial \psi\left(q_{\alpha_{1} \cdots \alpha_{r}}\right)}{\partial q_{\alpha_{1} \cdots \alpha_{r}}}=0
$$

necessária (embora não suficiente) para encontrar seus pontos de mínimo, requer um conjunto de $\left(\begin{array}{l}n \\ r\end{array}\right)$ equações representadas por

$$
q_{\alpha_{1} \cdots \alpha_{r}}=\frac{1}{Z_{n G}} \operatorname{Tr}_{\left\{\sigma_{\alpha}\right\}} \sigma_{\alpha_{1}} \cdots \sigma_{\alpha_{r}} \exp \left[G\left(\left\{\sigma_{\alpha}\right\}\right)+\ln \int_{\mathbb{R}} d H p_{H}(H) e^{\beta H \sum_{\alpha=1}^{n} \sigma_{\alpha}}\right]
$$

onde

$$
Z_{n G}:=\operatorname{Tr}_{\left\{\sigma_{\alpha}\right\}} \exp \left[G\left(\left\{\sigma_{\alpha}\right\}\right)+\ln \int_{\mathbb{R}} d H p_{H}(H) e^{\beta H \sum_{\alpha=1}^{n} \sigma_{\alpha}}\right]
$$

e

$$
G\left(\left\{\sigma_{\alpha}\right\}\right):=c \sum_{r=0}^{n} \sum_{\left(\alpha_{1}, \cdots, \alpha_{r}\right)} b_{r} q_{\alpha_{1} \cdots \alpha_{r}} \sigma_{\alpha_{1}} \cdots \sigma_{\alpha_{r}}
$$

\section{B.2- Equação auxiliar - I}

Considere

$$
e^{\sum_{\alpha=1}^{n} y_{\alpha} \sigma_{\alpha}}=\sum_{r=0}^{n} \sum_{\left(\alpha_{1}, \cdots, \alpha_{r}\right)} a_{\alpha_{1} \cdots \alpha_{r}} \sigma_{\alpha_{1}} \cdots \sigma_{\alpha_{r}}
$$

que é uma equação similar a (B.10), com $\left\{\sigma_{\alpha}\right\}$ sendo o conjunto de spins tipo Ising. Da mesma forma que em (B.11), tem-se 


$$
a_{\alpha_{1} \cdots \alpha_{r}}=\frac{1}{2^{n}} \operatorname{Tr}_{\left\{\sigma_{\alpha}\right\}} \sigma_{\alpha_{1}} \cdots \sigma_{\alpha_{r}} e^{\sum_{\alpha=1}^{n} y_{\alpha} \sigma_{\alpha}}=\left[\prod_{i=1}^{r} \sinh y_{\alpha_{i}}\right]\left[\prod_{i=r+1}^{n} \cosh y_{\alpha_{i}}\right]
$$

Para $y_{\alpha_{i}}=\tanh ^{-1} X_{\alpha_{i}}$, com $X_{\alpha} \in[-1,1]$, mostra-se que $a_{\alpha_{1} \cdots \alpha_{r}}=\prod_{i=1}^{r} X_{\alpha_{i}} \prod_{j=1}^{n}\left(1-X_{\alpha_{j}}^{2}\right)^{-\frac{1}{2}}$ que, substituída em (B.26), chega-se a

$$
\sum_{r=0}^{n} \sum_{\left(\alpha_{1}, \cdots, \alpha_{r}\right)} X_{\alpha_{1}} \cdots X_{\alpha_{r}} \sigma_{\alpha_{1}} \cdots \sigma_{\alpha_{r}}=\exp \left[\sum_{\alpha=1}^{n} \sigma_{\alpha} \tanh ^{-1} X_{\alpha}+\frac{1}{2} \sum_{\alpha=1}^{n} \ln \left(1-X_{\alpha}^{2}\right)\right]
$$

\section{B.3- Campo efetivo - solução réplica - simétrica}

A introdução do campo efetivo $h$, com distribuição de probabilidades $P$, é feita através do parâmetro de ordem

$$
q_{\alpha_{1} \cdots \alpha_{r}}=\int_{\mathbb{R}} d h P(h) \frac{\operatorname{Tr}_{\left\{\sigma_{\alpha}\right\}} \sigma_{\alpha_{1}} \cdots \sigma_{\alpha_{\tau}} e^{\beta h \sum_{\alpha=1}^{n} \sigma_{\alpha}}}{\operatorname{Tr}_{\left\{\sigma_{\alpha}\right\}} e^{\beta h \sum_{\alpha=1}^{n} \sigma_{\alpha}}}
$$

que, ao ser comparada com (B.23), relaciona as duas grandezas de interese, $G$ e $P$,

$$
\int_{\mathbb{R}} \frac{d h P(h) e^{\beta h \sum_{\alpha=1}^{n} \sigma_{\alpha}}}{2^{n} \cosh ^{n}(\beta h)}=\frac{1}{Z_{n G}} \exp \left[G\left(\left\{\sigma_{\alpha}\right\}\right)+\ln \int_{\mathbb{R}} d H p_{H}(H) \exp \left(\beta H \sum_{\alpha=1}^{n} \sigma_{\alpha}\right)\right]
$$

Efetuando o traço em (B.29),

$$
q_{\alpha_{1} \cdots \alpha_{r}}=\int_{\mathbb{R}} d h P(h) \tanh ^{r}(\beta h)
$$

e substituindo-a, juntamente com (B.12) em (B.25), tem-se

$$
G\left(\left\{\sigma_{\alpha}\right\}\right)=c \int_{\mathbb{R}} d J \rho(J) \int_{\mathbb{R}} d h P(h) \cosh ^{n}(\beta J) \sum_{r=0}^{n} \sum_{\left(\alpha_{1}, \cdots, \alpha_{r}\right)}[\tanh (\beta J) \tanh (\beta h)]^{\tau} \sigma_{\alpha_{\mathbf{1}}} \cdots \sigma_{\alpha_{r}},
$$

que com a aplicação da fórmula (B.28) (aqui, $X_{\alpha_{i}}=\tanh (\beta J) \tanh (\beta h) \in[-1,1]$ ), vem

$$
\begin{aligned}
G\left(\left\{\sigma_{\alpha}\right\}\right)= & c \int_{\mathbb{R}} d J \rho(J) \int_{\mathbb{R}} d h P(h) \cosh ^{n}(\beta J) \times \\
& \times \exp \left\{\sum_{\alpha=1}^{n} \sigma_{\alpha} \tanh ^{-1}[\tanh (\beta J) \tanh (\beta h)]+\frac{n}{2} \ln \left[1-\tanh ^{2}(\beta J) \tanh ^{2}(\beta h)\right]\right\},
\end{aligned}
$$


onde fica evidenciado a dependência $G\left(\left\{\sigma_{\alpha}\right\}\right)=G(\hat{\sigma})$, com $\hat{\sigma}:=\sum_{\alpha=1}^{n} \sigma_{\alpha}$.

Obtidas as equações (B.30) e (B.33), o passo seguinte é tomar o limite $n \rightarrow 0$; antes, porém, é considerada a continuação analítica $\mathbb{Z} \ni \hat{\sigma} \rightarrow i y / \beta \in \mathbb{C}, y \in \mathbb{R}$. Estas duas operações levam (B.30) e (B.33) a, respectivamente,

$$
\int_{\mathbb{R}} d h P(h) e^{i y h}=\int_{\mathbb{R}} d H p_{H}(H) e^{G(i y / \beta)-c+i y H}
$$

e

$$
G\left(\frac{i y}{\beta}\right)=c \int_{\mathbb{R}} d J \rho(J) \int_{\mathbb{R}} d h P(h) \exp \left[\frac{i y}{\beta} \tanh ^{-1}[\tanh (\beta J) \tanh (\beta h)]\right]
$$

onde $\lim _{n \rightarrow 0} Z_{n G}=e^{c}$, calculado a seguir. A fim de simplificar a notação, definem-se $U_{1}(J, h):=$ $\tanh ^{-1}[\tanh (\beta J) \tanh (\beta h)]$ e $U_{2}(J, h):=\ln \cosh (\beta J)+\frac{1}{2} \ln \left[.1-\tanh ^{2}(\beta J) \tanh ^{2}(\beta h)\right]$ para determinar $Z_{n G}$ no limite $n \rightarrow 0$; usando (B.33) em (B.24), vem

$$
\begin{aligned}
Z_{G}:= & \lim _{n \rightarrow 0} Z_{n G}=\lim _{n \rightarrow 0} \operatorname{Tr}_{\left\{\sigma_{\alpha}\right\}} \exp \left[G\left(\left\{\sigma_{\alpha}\right\}\right)+\ln \int_{\mathbb{R}} d H p_{H}(H) e^{\beta H \sum_{\alpha=1}^{n} \sigma_{\alpha}}\right] \\
= & \lim _{n \rightarrow 0} \int_{\mathbb{R}} d H p_{H}(H) \operatorname{Tr}_{\left\{\sigma_{\alpha}\right\}} e^{\beta H \sum_{\alpha=1}^{n} \sigma_{\alpha}} \sum_{m=0}^{\infty} \frac{c^{m}}{m !}\left[\int_{\mathbb{R}} d J \rho(J) \times\right. \\
& \left.\times \int_{\mathbb{R}} d h P(h) e^{U_{1}(J, h) \sum_{\alpha=1}^{n} \sigma_{\alpha}+n U_{2}(J, h)}\right]^{m} \\
= & \left.\lim _{n \rightarrow 0} \int_{\mathbb{R}} d H p_{H}(H) \sum_{m=0}^{\infty} \frac{c^{m}}{m !} \int \operatorname{Tr}_{\sigma} \exp \left[\sigma\left(\beta H+\sum_{j=1}^{m} U_{1}\left(J_{j}, h_{j}\right)\right)+\sum_{j=1}^{m} U_{2}\left(J_{j}, h_{j}\right)\right]\right\}^{n} \times \\
& \times \prod_{j=1}^{m} d J_{j} \rho\left(J_{i}\right) d h_{j} P\left(h_{j}\right) \\
= & \int_{\mathbb{R}} d H p_{H}(H) \sum_{m=0}^{\infty} \frac{c^{m}}{m !} \int \prod_{j=1}^{m} d J_{j} \rho\left(J_{i}\right) d h_{j} P\left(h_{j}\right)=e^{c} .
\end{aligned}
$$

A escolha da continuação analítica $\hat{\sigma} \rightarrow i y / \beta$ possibilita introduzir uma transformada de Fourier em (B.34),

$$
P(h)=\int_{\mathbb{R}} d H p_{H}(H) \int_{\mathbb{R}} d y e^{-i y(h-H)} e^{G(i y / \beta)-c} .
$$




\section{B.4- Energia livre estacionária - solução réplica - simétrica}

A energia livre

$$
f(\beta)=\lim _{n \rightarrow 0} \frac{1}{\beta n}\left[\frac{c}{2}+\frac{c}{2} \sum_{r=0}^{n} \sum_{\left(\alpha_{1}, \cdots, \alpha_{r}\right)} b_{r} q_{\alpha_{1} \cdots \alpha_{r}}^{2}-\ln \int_{\mathbb{R}} d H p_{H}(H) \operatorname{Tr}_{\left\{\sigma_{\alpha}\right\}} e^{G\left(\left\{\sigma_{\alpha}\right\}\right)+\beta H \sum_{\alpha=1}^{n} \sigma_{\alpha}}\right]
$$

pode ser expressa em termos da distribuição do campo efetivo $P$. Reescrever-se-á, a seguir, o segundo e o terceiro termo dentro do colchetes da equação (B.38).

$\underline{\text { Segundo termo: }}$

$\operatorname{De}(\mathrm{B} .12)$ e (B.31),

$$
\begin{aligned}
\frac{c}{2} \sum_{r=0}^{n} \sum_{\left(\alpha_{1}, \cdots, \alpha_{r}\right)} b_{r} q_{\alpha_{1} \cdots \alpha_{r}}^{2}= & \frac{c}{2} \int_{\mathbb{R}} d J \rho(J) \int_{\mathbb{R}} d h P(h) \int_{\mathbb{R}} d h^{\prime} P\left(h^{\prime}\right) \cosh ^{n}(\beta J) \times \\
& \times \sum_{r=0}^{n} \underbrace{\sum_{r}}_{\left(\begin{array}{c}
n \\
\left(\alpha_{1}, \cdots, \alpha_{r}\right)
\end{array}\right.} 1^{n-r}\left[\tanh (\beta J) \tanh (\beta h) \tanh \left(\beta h^{\prime}\right)\right]^{r} \\
= & \frac{c}{2} \int_{\mathbb{R}} d J \rho(J) \int_{\mathbb{R}} d h P(h) \int_{\mathbb{R}} d h^{\prime} P\left(h^{\prime}\right) \cosh ^{n}(\beta J) \times \\
& \times\left[1+\tanh (\beta J) \tanh (\beta h) \tanh \left(\beta h^{\prime}\right)\right]^{n} \\
= & \frac{c}{2} \int_{\mathbb{R}} d J \rho(J) \int_{\mathbb{R}} d h P(h) \int_{\mathbb{R}} d h^{\prime} P\left(h^{\prime}\right)\{1+n \ln \cosh (\beta J)+ \\
& \left.+n \ln \left[1+\tanh (\beta J) \tanh (\beta h) \tanh \left(\beta h^{\prime}\right)\right]+\mathcal{O}\left(n^{2}\right)\right\} .
\end{aligned}
$$

\section{Terceiro termo:}

De (B.33), e lembrando que $|\hat{\sigma}| \leq n$, tem-se 


$$
\begin{aligned}
& \ln \int_{\mathbb{R}} d H p_{H}(H) \operatorname{Tr}_{\left\{\sigma_{\alpha}\right\}} e^{G\left(\left\{\sigma_{\alpha}\right\}\right)+\beta H \sum_{\alpha=1}^{n} \sigma_{\alpha}}= \\
& =\ln \int_{\mathbb{R}} d H p_{H}(H) \operatorname{Tr}_{\left\{\sigma_{\alpha}\right\}} \exp \left\{\beta H \sum_{\alpha=1}^{n} \sigma_{\alpha}+c \int_{\mathbb{R}} d J \rho(J) \int_{\mathbb{R}} d h P(h) \times\right. \\
& \left.\times \exp \left[\sum_{\alpha=1}^{n} \sigma_{\alpha} \tanh ^{-1}[\tanh (\beta J) \tanh (\beta h)]+n \ln \cosh (\beta J)+\frac{n}{2} \ln \left[1-\tanh ^{2}(\beta J) \tanh ^{2}(\beta h)\right]\right]\right\} \\
& =\ln \int_{\mathbb{R}} d H p_{H}(H) \operatorname{Tr}_{\left\{\sigma_{\alpha}\right\}} \exp \left\{\beta H \hat{\sigma}+c \int_{\mathbb{R}} d J \rho(J) \int_{\mathbb{R}} d h P(h)\left[1+\hat{\sigma} \tanh ^{-1}[\tanh (\beta J) \tanh (\beta h)]+\right.\right. \\
& \left.\left.+\mathcal{O}\left(n^{2}\right)\right]\left[1+n \ln \cosh (\beta J)+\frac{n}{2} \ln \left[1-\tanh ^{2}(\beta J) \tanh ^{2}(\beta h)\right]+\mathcal{O}\left(n^{2}\right)\right]\right\} \\
& =\ln \int_{\mathbb{R}} d H p_{H}(H) \operatorname{Tr}_{\left\{\sigma_{\alpha}\right\}} \exp \left\{\beta H \hat{\sigma}+c+c \int_{\mathbb{R}} d J \rho(J) \int_{\mathbb{R}} d h P(h)\left[\hat{\sigma} \tanh ^{-1}[\tanh (\beta J) \tanh (\beta h)]+\right.\right. \\
& \left.\left.+n \ln \cosh (\beta J)+\frac{n}{2} \ln \left[1-\tanh ^{2}(\beta J) \tanh ^{2}(\beta h)\right]+\mathcal{O}\left(n^{2}\right)\right]\right\} \\
& =n c \int_{\mathbb{R}} d J \rho(J) \ln \cosh (\beta J)+\frac{n c}{2} \int_{\mathbb{R}} d J \rho(J) \int_{\mathbb{R}} d h P(h) \ln \left[1-\tanh ^{2}(\beta J) \tanh ^{2}(\beta h)\right]+\mathcal{O}\left(n^{2}\right)+ \\
& +\ln \int_{\mathbb{R}} d H p_{H}(H) \operatorname{Tr}_{\left\{\sigma_{\alpha}\right\}} \exp \left\{\beta H \hat{\sigma}+c \int_{\mathbb{R}} d J \rho(J) \int_{\mathbb{R}} d h P(h) e^{\hat{\sigma} \tanh ^{-1}[\tanh (\beta J) \tanh (\beta h)]}\right\} .
\end{aligned}
$$

A última linha de (B.40) pode ainda ser desenvolvida e apresentar um aspecto mais simples. Tomando $i y / \beta=s$ em (3.32), tem-se

$$
\int_{\mathbb{R}} d H p_{H}(H) e^{\beta s H+G(s)}=\int_{\mathbb{R}} d h P(h) e^{c+\beta s h} .
$$

Esta relação haverá de simplificar a forma final da energia livre estacionária na solução réplica-simétrica, visto que no passo seguinte a equação (B.41) será reconhecida dentro da estrutura da última linha de (B.40). Continuando com os cálculos, 


$$
\begin{aligned}
\ln \int_{\mathbb{R}} d H p_{H}(H) \operatorname{Tr}_{\left\{\sigma_{\alpha}\right\}} e^{G\left(\left\{\sigma_{\alpha}\right\}\right)+\beta H \sum_{\alpha=1}^{n} \sigma_{\alpha}}= \\
=\quad n c \int_{\mathbb{R}} d J \rho(J) \ln \cosh (\beta J)+\frac{n c}{2} \int_{\mathbb{R}} d J \rho(J) \int_{\mathbb{R}} d h P(h) \ln \left[1-\tanh ^{2}(\beta J) \tanh ^{2}(\beta h)\right]+ \\
\quad \mathcal{O}\left(n^{2}\right)+\ln \operatorname{Tr}_{\left\{\sigma_{\alpha}\right\}} \int_{\mathbb{R}} d s \delta(s-\hat{\sigma}) \times \\
\quad \times \underbrace{}_{\mathbb{R}} d H p_{H}(H) \exp \left\{\beta H s+c \int_{\mathbb{R}} d J \rho(J) \int_{\mathbb{R}} d h P(h) e^{s \tanh ^{-1}[\tanh (\beta J) \tanh (\beta h)]}\right\} \\
=\quad n c \int_{\mathbb{R}} d J \rho(J) \ln \cosh (\beta J)+\frac{n c}{2} \int_{\mathbb{R}} d J \rho(J) \int_{\mathbb{R}} d h P(h) \ln \left[1-\tanh ^{2}(\beta J) \tanh ^{2}(\beta h)\right]+\mathcal{O}\left(n^{2}\right)+ \\
\quad+\ln \operatorname{Tr}_{\left\{\sigma_{\alpha}\right\}} \int_{\mathbb{R}} d h P(h) e^{c+\beta \hat{\sigma} h} \\
\quad n c \int_{\mathbb{R}} d J \rho(J) \ln \cosh (\beta J)+\frac{n c}{2} \int_{\mathbb{R}} d J \rho(J) \int_{\mathbb{R}} d h P(h) \ln \left[1-\tanh ^{2}(\beta J) \tanh ^{2}(\beta h)\right]+ \\
+c+\ln \int_{\mathbb{R}} d h P(h)[2 \cosh (\beta h)]^{n}+\mathcal{O}\left(n^{2}\right) .
\end{aligned}
$$

A energia livre estacionária em termos de $P$ é obtida reunindo (B.39) e (B.42) em (B.38), e tomando o limite $n \rightarrow 0$,

$$
\begin{aligned}
f(\beta)= & -\frac{c}{2 \beta} \int_{\mathbb{R}} d J \rho(J) \ln \cosh (\beta J)-\int_{\mathbb{R}} d h P(h) \ln [2 \cosh (\beta h)]+ \\
& +\frac{c}{2 \beta} \int_{\mathbb{R}} d J \rho(J) \int_{\mathbb{R}} d h P(h) \int_{\mathbb{R}} d h^{\prime} P\left(h^{\prime}\right) \ln \left[1+\tanh (\beta J) \tanh (\beta h) \tanh \left(\beta h^{\prime}\right)\right]- \\
& -\frac{c}{2 \beta} \int_{\mathbb{R}} d J \rho(J) \int_{\mathbb{R}} d h P(h) \ln \left[1-\tanh ^{2}(\beta J) \tanh ^{2}(\beta h)\right]
\end{aligned}
$$

\section{B.5- Relações auxiliares no estado fundamental}

$$
\lim _{\beta \rightarrow \infty} \frac{1}{\beta} \tanh ^{-1}[\tanh (\beta a) \tanh (\beta b)]=\operatorname{sign}(a b) \min \{|a|,|b|\}
$$




$$
\begin{aligned}
& \lim _{\beta \rightarrow \infty} \frac{1}{\beta} \tanh ^{-1}[\tanh (\beta a) \tanh (\beta b)]= \operatorname{sign}(a b) \lim _{\beta \rightarrow \infty} \frac{1}{\beta} \tanh ^{-1}[\tanh (\beta|a|) \tanh (\beta|b|)] \\
&= \operatorname{sign}(a b) \lim _{\beta \rightarrow \infty} \frac{1}{2 \beta} \ln \left[\frac{1+\tanh (\beta|a|) \tanh (\beta|b|)}{1-\tanh (\beta|a|) \tanh (\beta|b|)}\right] \\
&= \operatorname{sign}(a b) \lim _{\beta \rightarrow \infty} \frac{1}{2 \beta} \ln \left[\frac{e^{2 \beta|a|+2 \beta|b|}+1}{\left.e^{2 \beta|a|}+e^{2 \beta|b|}\right]}\right. \\
&= \operatorname{sign}(a b) \lim _{\beta \rightarrow \infty} \frac{1}{2 \beta}\left[2 \beta|a|+2 \beta|b|+\mathcal{O}\left(e^{-2 \beta|a|-2 \beta|b|}\right)-\right. \\
&=\left.-2 \beta \max \{|a|,|b|\}-\mathcal{O}\left(e^{-2 \beta|| a|-| b||}\right)\right] \\
& \lim _{\beta \rightarrow \infty}\left[\frac{\cosh (\beta a+\beta J) \cosh (\beta a-\beta J)}{\cosh (\beta b+\beta J) \cosh (\beta b-\beta J)}\right]^{\frac{1}{\beta}}=\exp [2 \max \{|a|,|J|\}-2 \max \{|b|,|J|\}] \\
& \lim _{\beta \rightarrow \infty}\left[\frac{\cosh (\beta a+\beta J) \cosh (\beta a-\beta J)}{\cosh (\beta b+\beta J) \cosh (\beta b-\beta J)}\right]^{\frac{1}{\beta}}= \lim _{\beta \rightarrow \infty}\left[\frac{e^{2 \beta a}+e^{2 \beta J}+e^{-2 \beta J}+e^{-2 \beta a}}{e^{2 \beta b}+e^{2 \beta J}+e^{-2 \beta J}+e^{-2 \beta b}}\right]^{\frac{1}{\beta}} \\
&= \lim _{\beta \rightarrow \infty}\left[\frac{e^{2 \beta|a|}+e^{2 \beta|J|}+\mathcal{O}\left(e^{-2 \beta|J|}\right)+\mathcal{O}\left(e^{-2 \beta|a|}\right)}{e^{2 \beta|b|}+e^{2 \beta|J|}+\mathcal{O}\left(e^{-2 \beta|J|}\right)+\mathcal{O}\left(e^{-2 \beta|b|}\right)}\right]^{\frac{1}{\beta}} \\
&= \lim _{\beta \rightarrow \infty}\left[\frac{e^{2 \beta \max \{|a|,\{J \mid\}}+\mathcal{O}\left(e^{-2 \beta|| a|-| J \mid}\right)}{e^{2 \beta \max \{|b|,|J|\}}+\mathcal{O}\left(e^{-2 \beta|| b|-| J||}\right)}\right]^{\frac{1}{\beta}} \\
&= \exp [2 \max \{|a|,|J|\}-2 \max \{|b|,|J|\}]
\end{aligned}
$$

\section{B.6- Coeficientes $A, B, C$ e $\left\{a_{k}\right\}$}

No contexto da solução réplica-simétrica, e para campos aleatórios $H_{R}$ múltiplos de $J_{0}(m:=$ $H_{R} / J_{0} \in \mathbb{Z}_{+}$), a distribuição de campo efetivo $P$ é representada por uma soma

$$
P(h)=\sum_{k \in \mathbb{Z}} a_{k} \delta\left(h-k J_{0}\right)
$$

Viu-se, ainda, que

$$
\tilde{G}(i x):=\lim _{\beta \rightarrow \infty} G\left(\frac{i x}{\beta}\right)=A+B e^{i x J_{0}}+C e^{-i x J_{0}}
$$

onde

$$
A=c a_{0} \quad, \quad B=c \sum_{k=1}^{\infty} a_{k} \quad \text { e } \quad C=c \sum_{k=-\infty}^{-1} a_{k}
$$


A não-negatividade de $P$, que é uma função distribuição de probabilidades $(P(h) \geq 0, \forall h \in \mathbb{R})$, implica $a_{k} \geq 0$ para todo $k \in \mathbb{Z}$ e, por conseguinte, $A, B$ e $C$ são todos não-negativos. B.5)

$\mathrm{De}$ (3.35) e (3.32) no estado fundamental, tem-se, invocando (B.2), (B.3), (3.31) e (B.44) (Apêndice

$$
\begin{aligned}
\tilde{G}(i x) & =A+B e^{i x J_{0}}+C e^{-i x J_{0}} \\
& =\frac{c}{2} \int_{\mathbb{R}} d y\left(e^{i y m J_{0}}+e^{-i y m J_{0}}\right) \int_{\mathbb{R}} d h \exp \left[-i y h+\tilde{G}(i y)-c+i x \operatorname{sign}(h) \min \left\{J_{0},|h|\right\}\right] \\
& =\frac{c e^{A-c}}{2} \int_{\mathbb{R}} d h e^{i x \operatorname{sign}(h) \min \left\{J_{0},|h|\right\}} \int_{\mathbb{R}} d y e^{-i y h}\left(e^{i y m J_{0}}+e^{-i y m J_{0}}\right) \sum_{r=0}^{\infty} \frac{B^{r}}{r !} e^{i y r J_{0}} \sum_{s=0}^{\infty} \frac{C^{s}}{s !} e^{-i y s J_{0}} \\
& =\frac{c e^{A-c}}{2} \sum_{r=0}^{\infty} \sum_{s=0}^{\infty} \frac{B^{r} C^{s}}{r ! s !}\left[e^{i x J_{0} \operatorname{sign}(m+r-s) \min \{1,|m+r-s|\}}+e^{i x J_{0} \operatorname{sign}(-m+r-s) \min \{1,|-m+r-s|\}}\right],
\end{aligned}
$$

donde se nota que

$$
\tilde{G}(0)=c=A+B+C
$$

Da primeira e última linha de (B.51), obtêm-se

$$
\begin{aligned}
& A=\frac{c e^{A-c}}{2}\left[\sum_{r=0}^{\infty} \frac{B^{r} C^{r+m}}{r !(r+m) !}+\sum_{s=0}^{\infty} \frac{B^{s+m} C^{s}}{(s+m) ! s !}\right] \\
& B=\frac{c e^{A-c}}{2}\left[\sum_{\substack{r, s=0 \\
m+r-s \geq 1}}^{\infty} \frac{B^{r} C^{s}}{r ! s !}+\sum_{\substack{r, s=0 \\
-m+r-s \geq 1}}^{\infty} \frac{B^{r} C^{s}}{r ! s !}\right]
\end{aligned}
$$

e

$$
C=\frac{c e^{A-c}}{2}\left[\sum_{\substack{r, s=0 \\ m+r-s \leq-1}}^{\infty} \frac{B^{r} C^{s}}{r ! s !}+\sum_{\substack{r, s=0 \\-m+r-s \leq-1}}^{\infty} \frac{B^{r} C^{s}}{r ! s !}\right]
$$

A equação (B.52) permite dispensar uma das equações acima; escolheu-se, no presente trabalho, não considerar (B.55).

As três equações, (B.52), (B.53) e (B.54), no contexto da solução réplica-simétrica, determinam $A$, $B$ e $C$, dados os parâmetros de conectividade $c$ e a razão $m:=H_{R} / J_{0} \in \mathbb{Z}_{+}$. Antes de prosseguir, um resultado auxiliar será fornecido para reescrever duas dessas equações. Integrando por partes sucessivamente a integral

$$
\int_{0}^{x} d t f^{(p+1)}(t) \frac{(x-t)^{p}}{p !}
$$


onde $f^{(p+1)}$ indica a $(p+1)$-ésima derivada de $f$, tem-se

$$
\int_{0}^{x} d t f^{(p+1)}(t) \frac{(x-t)^{p}}{p !}=f(x)-\sum_{k=0}^{p} f^{(k)}(0) \frac{x^{k}}{k !}
$$

A escolha $f(x)=e^{x}$ implica

$$
\sum_{k=0}^{p} \frac{x^{k}}{k !}=e^{x}-x^{p+1} \int_{0}^{1} d u e^{x u} \frac{(1-u)^{p}}{p !}
$$

ou

$$
\sum_{k=p+1}^{\infty} \frac{x^{k}}{k !}=x^{p+1} \int_{0}^{1} d u e^{x u} \frac{(1-u)^{p}}{p !}
$$

que serão usadas mais adiante. A primeira equação, (B.53), pode ser escrita, via funções de Bessel modificadas,

$$
I_{\nu}(z):=\sum_{k=0}^{\infty} \frac{\left(\frac{z}{2}\right)^{2 k+\nu}}{\Gamma(k+\nu+1) k !}
$$

como

$$
A=\frac{c e^{A-c}}{2}\left[\left(\frac{B}{C}\right)^{\frac{m}{2}}+\left(\frac{B}{C}\right)^{-\frac{m}{2}}\right] I_{m}(2 \sqrt{B C}),
$$

lembrando que $I_{\nu}(z)=I_{-\nu}(z)$ para $\nu \in \mathbb{Z}$.

Desenvolvendo a segunda equação, (B.54), invocando as relações (B.58) e (B.59), obtidas anteriormente, e supondo, inicialmente, que $m \geq 1$, tem-se

$$
\begin{aligned}
B= & \frac{c e^{A-c}}{2}\left[\sum_{\substack{r, s=0 \\
m+r-s \geq 1}}^{\infty} \frac{B^{r} C^{r}}{r ! s !}+\sum_{\substack{r, s=0 \\
-m+r-s \geq 1}}^{\infty} \frac{B^{r} C^{r}}{r ! s !}\right] \\
= & \frac{c e^{A-c}}{2}\left[\sum_{r=0}^{\infty} \frac{B^{r}}{r !} \sum_{s=0}^{m+r-1} \frac{C^{s}}{s !}+\sum_{s=0}^{\infty} \frac{C^{s}}{s !} \sum_{r=m+s+1}^{\infty} \frac{B^{r}}{r !}\right] \\
= & e^{B+C}-C\left(\frac{C}{B}\right)^{\frac{m-1}{2}} \int_{0}^{1} d u e^{C u}(1-u)^{\frac{m-1}{2}} I_{m-1}(2 \sqrt{B C(1-u)})+ \\
& +B\left(\frac{B}{C}\right)^{\frac{m}{2}} \int_{0}^{1} d u e^{B u}(1-u)^{\frac{m}{2}} I_{m}(2 \sqrt{B C(1-u)})
\end{aligned}
$$


Para $m=0$, tem-se

$$
\begin{aligned}
B= & \frac{c e^{A-c}}{2}\left[\sum_{\substack{r, s=0 \\
r-s \geq 1}}^{\infty} \frac{B^{r} C^{r}}{r ! s !}+\sum_{\substack{r, s=0 \\
r-s \geq 1}}^{\infty} \frac{B^{r} C^{r}}{r ! s !}\right] \\
= & \frac{c e^{A-c}}{2}\left[\sum_{r=1}^{\infty} \frac{B^{r}}{r !} \sum_{s=0}^{r-1} \frac{C^{s}}{s !}+\sum_{s=0}^{\infty} \frac{C^{s}}{s !} \sum_{r=s+1}^{\infty} \frac{B^{r}}{r !}\right] \\
= & e^{B+C}-e^{C}-C \sqrt{\frac{B}{C}} \int_{0}^{1} d u e^{C u}(1-u)^{-\frac{1}{2}} I_{1}(2 \sqrt{B C(1-u)})+ \\
& +B \int_{0}^{1} d u e^{B u} I_{0}(2 \sqrt{B C(1-u)}) .
\end{aligned}
$$

Reunindo (B.62) e (B.63), chega-se, finalmente, a

$$
\begin{aligned}
B= & e^{B+C}-e^{C} \delta_{m, 0}-C\left(\frac{C}{B}\right)^{\frac{m-1}{2}} \int_{0}^{1} d u e^{C u}(1-u)^{\frac{m-1}{2}} I_{m-1}(2 \sqrt{B C(1-u)})+ \\
& +B\left(\frac{B}{C}\right)^{\frac{m}{2}} \int_{0}^{1} d u e^{B u}(1-u)^{\frac{m}{2}} I_{m}(2 \sqrt{B C(1-u)})
\end{aligned}
$$

A determinação do conjunto $\left\{a_{k}\right\}$ consiste em desenvolver (B.37) no estado fundamental usando (B.49) e comparar termo a termo com (B.48),

$$
\begin{aligned}
P(h)= & \sum_{k \in \mathbb{Z}} a_{k} \delta\left(h-k J_{0}\right) \\
= & \int_{\mathbb{R}} d H p_{H}(H) e^{-i y(h-H)} e^{\tilde{G}(i y)-c} \\
= & \int_{\mathbb{R}} d y e^{-i y h}\left[\frac{e^{i y m J_{0}}+e^{-i y m J_{0}}}{2}\right] e^{A+B e^{i y J_{0}}+C e^{-i y J_{0}}-c} \\
= & \frac{e^{A-c}}{2} \sum_{r=0}^{\infty} \sum_{s=0}^{\infty} \frac{B^{r} C^{s}}{r ! s !} \int_{\mathbb{R}} d y e^{-i y h}\left(e^{i y m J_{0}}+e^{-i y m J_{0}}\right) e^{i y(r-s) J_{0}} \\
= & \frac{e^{A-c}}{2} \sum_{r=0}^{\infty} \sum_{s=0}^{\infty} \frac{B^{r} C^{s}}{r ! s !} \delta\left(h-r J_{0}+s J_{0}-m J_{0}\right)+ \\
& +\frac{e^{A-c}}{2} \sum_{r=0}^{\infty} \sum_{s=0}^{\infty} \frac{B^{r} C^{s}}{r ! s !} \delta\left(h-r J_{0}+s J_{0}+m J_{0}\right) .
\end{aligned}
$$

Definindo $k:=r-s-m$ para a primeira série acima e $k:=r-s+m$ para a segunda, e com (B.60), pode-se escrever 


$$
\begin{aligned}
P(h)= & \frac{e^{A-c}}{2}\left[\sum_{k-m \geq 0} \sum_{s=0}^{\infty} \frac{B^{k-m+s} C^{s}}{(k-m+s) ! s !} \delta\left(h-k J_{0}\right)+\sum_{k-m<0} \sum_{r=0}^{\infty} \frac{B^{r} C^{r+m-k}}{r !(r+m-k) !} \delta\left(h-k J_{0}\right)\right]+ \\
& +\frac{e^{A-c}}{2}\left[\sum_{k+m \geq 0} \sum_{s=0}^{\infty} \frac{B^{k+m+s} C^{s}}{(k+m+s) ! s !} \delta\left(h-k J_{0}\right)+\sum_{k+m<0} \sum_{r=0}^{\infty} \frac{B^{r} C^{r-m-k}}{r !(r-m-k) !} \delta\left(h-k J_{0}\right)\right] \\
= & \frac{e^{A-c}}{2}\left\{\sum_{k-m \geq 0}\left(\frac{B}{C}\right)^{\frac{k-m}{2}} I_{k-m}(2 \sqrt{B C}) \delta\left(h-k J_{0}\right)+\right. \\
& +\sum_{k-m<0}\left(\frac{B}{C}\right)^{\frac{k-m}{2}} I_{k-m}(2 \sqrt{B C}) \delta\left(h-k J_{0}\right)+\sum_{k+m \geq 0}\left(\frac{B}{C}\right)^{\frac{k+m}{2}} I_{k+m}(2 \sqrt{B C}) \delta\left(h-k J_{0}\right)+ \\
& \left.+\sum_{k+m<0}\left(\frac{B}{C}\right)^{\frac{k+m}{2}} I_{k+m}(2 \sqrt{B C}) \delta\left(h-k J_{0}\right)\right\} \\
= & \sum_{k \in \mathbb{Z}} \frac{e^{A-c}}{2}\left[\left(\frac{B}{C}\right)^{\frac{k+m}{2}} I_{k+m}(2 \sqrt{B C})+\left(\frac{B}{C}\right)^{\frac{k-m}{2}} I_{k-m}(2 \sqrt{B C})\right] \delta\left(h-k J_{0}\right) ;
\end{aligned}
$$

da primeira linha de (B.65), vê-se que

$$
a_{k}=\frac{e^{A-c}}{2}\left[\left(\frac{B}{C}\right)^{\frac{k+m}{2}} I_{k+m}(2 \sqrt{B C})+\left(\frac{B}{C}\right)^{\frac{k-m}{2}} I_{k-m}(2 \sqrt{B C})\right]
$$

\section{B.7- Equação secular}

Dado o funcional $\psi[G]$,

$$
\begin{aligned}
\psi[G]= & \frac{c}{2}+\frac{1}{2 c 2^{2 n}} \sum_{r=0}^{n} \sum_{\left(\alpha_{1}, \cdots, \alpha_{r}\right)} \frac{1}{b_{r}} \operatorname{Tr}_{\left\{\sigma_{\alpha}\right\}} \operatorname{Tr}_{\left\{\tau_{\alpha}\right\}} G\left(\left\{\sigma_{\alpha}\right\}\right) G\left(\left\{\tau_{\alpha}\right\}\right) \sigma_{\alpha_{1}} \cdots \sigma_{\alpha_{r}} \tau_{\alpha_{1}} \cdots \tau_{\alpha_{r}}- \\
& -\ln \operatorname{Tr}_{\left\{\sigma_{\alpha}\right\}} \int_{\mathbb{R}} d H p_{H}(H) e^{G\left(\left\{\sigma_{\alpha}\right\}\right)+\beta H \sum_{\alpha=1}^{n} \sigma_{\alpha}}
\end{aligned}
$$

sua primeira derivada funcional é

$$
\begin{aligned}
\frac{\delta f[G]}{\delta G\left(\left\{\sigma_{\alpha}\right\}\right)}= & \frac{1}{c 2^{2 n}} \sum_{r=0}^{n} \sum_{\left(\alpha_{1}, \cdots, \alpha_{r}\right)} \frac{1}{b_{r}} \operatorname{Tr}_{\left\{\tau_{\alpha}\right\}} G\left(\left\{\tau_{\alpha}\right\}\right) \sigma_{\alpha_{1}} \cdots \sigma_{\alpha_{r}} \tau_{\alpha_{1}} \cdots \tau_{\alpha_{r}}- \\
& -\frac{1}{Z_{n G}} \int_{\mathbb{R}} d H p_{H}(H) e^{G\left(\left\{\sigma_{\alpha}\right\}\right)+\beta H \sum_{\alpha=1}^{n} \sigma_{\alpha}}
\end{aligned}
$$

enquanto a sua segunda derivada funcional é 


$$
\begin{aligned}
\frac{\delta^{2} f[G]}{\delta G\left(\left\{\sigma_{\alpha}\right\}\right) \delta G\left(\left\{\tau_{\alpha}\right\}\right)}= & \frac{1}{c 2^{2 n}} \sum_{r=0}^{n} \sum_{\left(\alpha_{1}, \cdots, \alpha_{r}\right)} \frac{1}{b_{r}} \sigma_{\alpha_{1}} \cdots \sigma_{\alpha_{r}} \tau_{\alpha_{1}} \cdots \tau_{\alpha_{r}}+ \\
& +\int_{\mathbb{R}} d H p_{H}(H) \iint_{\mathbb{R}} d H^{\prime} p_{H}\left(H^{\prime}\right) \frac{e^{G\left(\left\{\sigma_{\alpha}\right\}\right)+\beta H \sum_{\alpha=1}^{n} \sigma_{\alpha}}}{Z_{n G}} \frac{e^{G\left(\left\{\tau_{\alpha}\right\}\right)+\beta H^{\prime} \sum_{\alpha=1}^{n} \tau_{\alpha}}}{Z_{n G}}- \\
& -\int_{\mathbb{R}} d H p_{H}(H) \frac{e^{G\left(\left\{\sigma_{\alpha}\right\}\right)+\beta H \sum_{\alpha=1}^{n} \sigma_{\alpha}}}{Z_{n G}} \delta_{\left\{\sigma_{\alpha}\right\},\left\{\tau_{\alpha}\right\}}
\end{aligned}
$$

onde $\delta_{\left\{\sigma_{\alpha}\right\},\left\{\tau_{\alpha}\right\}}:=\prod_{\alpha=1}^{n} \delta_{\sigma_{\alpha}, \tau_{\alpha}}$. Substituindo (B.70) na equação secular

$$
\operatorname{Tr}_{\left\{\tau_{\alpha}\right\}} \frac{\delta^{2} \psi[G]}{\delta G\left(\left\{\sigma_{\alpha}\right\}\right) \delta G\left(\left\{\tau_{\alpha}\right\}\right)} \varphi\left(\left\{\tau_{\alpha}\right\}\right)=\lambda \varphi\left(\left\{\sigma_{\alpha}\right\}\right)
$$

é imediato que

$$
\begin{aligned}
\lambda \varphi\left(\left\{\sigma_{\alpha}\right\}\right)= & \frac{1}{c 2^{2 n}} \sum_{r=0}^{n} \sum_{\left(\alpha_{1}, \cdots, \alpha_{r}\right)} \frac{1}{b_{r}} \sigma_{\alpha_{1}} \cdots \sigma_{\alpha_{r}} \operatorname{Tr}_{\left\{\tau_{\alpha}\right\}} \tau_{\alpha_{1}} \cdots \tau_{\alpha_{r}} \varphi\left(\left\{\tau_{\alpha}\right\}\right)+ \\
& +\int_{\mathbb{R}} d H p_{H}(H) \int_{\mathbb{R}} d H^{\prime} p_{H}\left(H^{\prime}\right) \frac{e^{G\left(\left\{\sigma_{\alpha}\right\}\right)+\beta H \sum_{\alpha=1}^{n} \sigma_{\alpha}}}{Z_{n G}} \operatorname{Tr}_{\left\{\tau_{\alpha}\right\}} \frac{e^{G\left(\left\{\tau_{\alpha}\right\}\right)+\beta H^{\prime} \sum_{\alpha=1}^{n} \tau_{\alpha}}}{Z_{n G}} \varphi\left(\left\{\tau_{\alpha}\right\}\right)- \\
& -\int_{\mathbb{R}} d H p_{H}(H) \frac{e^{G\left(\left\{\sigma_{\alpha}\right\}\right)+\beta H \sum_{\alpha=1}^{n} \sigma_{\alpha}}}{Z_{n G}} \operatorname{Tr}_{\left\{\tau_{\alpha}\right\}} \delta_{\left\{\sigma_{\alpha}\right\},\left\{\tau_{\alpha}\right\}} \varphi\left(\left\{\tau_{\alpha}\right\}\right) .
\end{aligned}
$$

Por conveniência, multiplicam-se ambos membros da equação (B.72) por $e^{\beta J \sum_{\alpha=1}^{n} \sigma_{\alpha} s_{\alpha}}$, onde $s_{\alpha}$ também é um spin de Ising para cada $\alpha \in\{1, \cdots, n\}$; em seguida, toma-se a esperança em relação à medida de probabilidade $d J \rho(J)$ para, finalmente, tomar o traço em $\left\{\sigma_{\alpha}\right\}$ nos dois membros da equação. Efetuando estas operações (lineares) termo a termo em (B.72), vem

Primeiro membro:

$$
\lambda \operatorname{Tr}_{\left\{\sigma_{\alpha}\right\}} \int_{\mathbb{R}} d J \rho(J) e^{\beta J \sum_{\alpha=1}^{n} \sigma_{\alpha} s_{\alpha}} \varphi\left(\left\{\sigma_{\alpha}\right\}\right) .
$$

Segundo membro (primeiro termo):

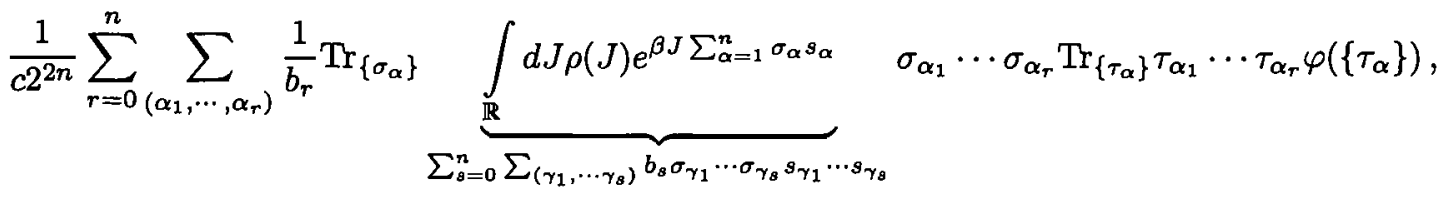

onde a expansão (B.10) foi invocada. Então,

$$
\frac{1}{c 2^{2 n}} \sum_{r=0}^{n} \sum_{\left(\alpha_{1}, \cdots, \alpha_{r}\right)} \frac{1}{b_{r}} \sum_{s=0}^{n} \sum_{\left(\gamma_{1}, \cdots \gamma_{s}\right)} \dot{b_{s} s_{\gamma_{1}} \cdots s_{\gamma_{s}}} \operatorname{Tr}_{\left\{\tau_{\alpha}\right\}} \tau_{\alpha_{1}} \cdots \tau_{\alpha_{r}} \varphi\left(\left\{\tau_{\alpha}\right\}\right) \underbrace{\operatorname{Tr}_{\left\{\sigma_{\alpha}\right\}} \sigma_{\alpha_{1}} \cdots \sigma_{\alpha_{r}} \sigma_{\gamma_{1}} \cdots \sigma_{\gamma_{s}}}_{\delta_{r, s} \delta_{\left(\alpha_{1}, \cdots, \alpha_{r}\right),\left(\gamma_{1}, \cdots, \gamma_{s}\right)}},
$$


sendo que

$$
\delta_{\left(\alpha_{1}, \cdots, \alpha_{r}\right),\left(\gamma_{1}, \cdots, \gamma_{s}\right)}:= \begin{cases}\prod_{k=1}^{r} \delta_{\alpha_{k}, \gamma_{k}} & , \quad r=s \\ 0 & , \quad r \neq s\end{cases}
$$

sempre considerando $\alpha_{1}<\cdots<\alpha_{r}$ e $\gamma_{1}<\cdots<\gamma_{s}$. Prosseguindo com os cálculos, (B.75) torna-se

$$
\begin{aligned}
& \frac{1}{c 2^{2 n}} \sum_{r=0}^{n} \sum_{\left(\alpha_{1}, \cdots, \alpha_{r}\right)} \frac{1}{b_{r}} 2^{n} b_{r} s_{\alpha_{1}} \cdots s_{\alpha_{r}} \operatorname{Tr}_{\left\{\tau_{\alpha}\right\}} \tau_{\alpha_{1}} \cdots \tau_{\alpha_{r}} \varphi\left(\left\{\tau_{\alpha}\right\}\right)= \\
& =\frac{1}{c 2^{n}} \operatorname{Tr}_{\left\{\tau_{\alpha}\right\}} \varphi\left(\left\{\tau_{\alpha}\right\}\right) \underbrace{}_{\prod_{r=0}^{\left.\sum_{\prod_{\alpha=1}^{n}\left(\alpha_{1}, \cdots, \alpha_{r}\right)}^{n} \sum_{\alpha_{\alpha}} \tau_{\alpha}\right)=2^{n} \prod_{\alpha_{1}}^{n} \cdots s_{\alpha_{r}} \delta_{\alpha_{\alpha}, \tau_{\alpha}}}} s_{\alpha_{r}} \\
& =\frac{1}{c} \varphi\left(\left\{s_{\alpha}\right\}\right) .
\end{aligned}
$$

$\underline{\text { Segundo membro (segundo termo): }}$

$$
\begin{aligned}
& \operatorname{Tr}_{\left\{\sigma_{\alpha}\right\}} \underbrace{\int_{\sum_{\left(\alpha_{1}, \cdots \alpha_{r}\right)} b_{r} \sigma_{\alpha_{1}} \cdots \sigma_{\alpha_{r} s_{\alpha_{1}} \cdots s_{\alpha_{r}}}} d J \rho(J) e^{\beta J \sum_{\alpha=1}^{n} \sigma_{\alpha} s_{\alpha}}}_{\sum_{r=0}^{n}} \int_{\mathbb{R}} d H p_{H}(H) \frac{e^{G\left(\left\{\sigma_{\alpha}\right\}\right)+\beta H \sum_{\alpha=1}^{n} \sigma_{\alpha}}}{Z_{n G}} \times \\
& \times \int_{\mathbb{R}} d H^{\prime} p_{H}\left(H^{\prime}\right) \operatorname{Tr}_{\left\{\tau_{\alpha}\right\}} \frac{e^{G\left(\left\{\tau_{\alpha}\right\}\right)+\beta H^{\prime} \sum_{\alpha=1}^{n} \tau_{\alpha}}}{Z_{n G}} \varphi\left(\left\{\tau_{\alpha}\right\}\right)= \\
& =\sum_{r=0}^{n} \sum_{\left(\alpha_{1}, \cdots \alpha_{r}\right)} b_{r} s_{\alpha_{1}} \cdots s_{\alpha_{r}} \underbrace{\frac{1}{Z_{n G}} \int_{\mathbb{R}} d H p_{H}(H) \operatorname{Tr}_{\left\{\sigma_{\alpha}\right\}} \sigma_{\alpha_{1}} \cdots \sigma_{\alpha_{r}} e^{G\left(\left\{\sigma_{\alpha}\right\}\right)+\beta H \sum_{\alpha=1}^{n} \sigma_{\alpha}} \times} \\
& \underbrace{q_{\alpha_{1} \cdots \alpha_{r}}^{\alpha_{\alpha}}}_{\frac{G\left(\left\{\beta_{\alpha}\right\}\right)}{c}} \\
& \times \int_{\mathbb{R}} d H^{\prime} p_{H}\left(H^{\prime}\right) \operatorname{Tr}_{\left\{\tau_{\alpha}\right\}} \frac{e^{G\left(\left\{\tau_{\alpha}\right\}\right)+\beta H^{\prime} \sum_{\alpha=1}^{n} \tau_{\alpha}}}{Z_{n G}} \varphi\left(\left\{\tau_{\alpha}\right\}\right) \\
& =\frac{G\left(\left\{s_{\alpha}\right\}\right)}{c} \int_{\mathbb{R}} d H p_{H}(H) \operatorname{Tr}_{\left\{\sigma_{\alpha}\right\}} \frac{e^{G\left(\left\{\sigma_{\alpha}\right\}\right)+\beta H \sum_{\alpha=1}^{n} \sigma_{\alpha}}}{Z_{n G}} \varphi\left(\left\{\sigma_{\alpha}\right\}\right) .
\end{aligned}
$$

As equações (B.10), (B.23) e (B.25) foram usadas.

Segundo membro (terceiro termo):

$$
\begin{aligned}
& \operatorname{Tr}_{\left\{\sigma_{\alpha}\right\}} \int_{\mathbb{R}} d H p_{H}(H) \frac{e^{G\left(\left\{\sigma_{\alpha}\right\}\right)+\beta H \sum_{\alpha=1}^{n} \sigma_{\alpha}}}{Z_{n G}} \operatorname{Tr}_{\left\{\tau_{\alpha}\right\}} \int_{\mathbb{R}} d J \rho(J) e^{\beta J \sum_{\alpha=1}^{n} \sigma_{\alpha} s_{\alpha}} \delta_{\left\{\sigma_{\alpha}\right\},\left\{\tau_{\alpha}\right\}} \varphi\left(\left\{\tau_{\alpha}\right\}\right)= \\
& =\operatorname{Tr}_{\left\{\sigma_{\alpha}\right\}} \int_{\mathbb{R}} d H p_{H}(H) \frac{e^{G\left(\left\{\sigma_{\alpha}\right\}\right)+\beta H \sum_{\alpha=1}^{n} \sigma_{\alpha}}}{Z_{n G}} \int_{\mathbb{R}} d J \rho(J) e^{\beta J \sum_{\alpha=1}^{n} \sigma_{\alpha} s_{\alpha}} \varphi\left(\left\{\sigma_{\alpha}\right\}\right) .
\end{aligned}
$$


Reunindo (B.73), (B.77), (B.78) e (B.79) em (B.72), e lembrando que $G\left(\left\{\sigma_{\alpha}\right\}\right)=G(\hat{\sigma})$, tem-se a equação secular (3.44),

$$
\begin{aligned}
\lambda \operatorname{Tr}_{\left\{\sigma_{\alpha}\right\}} \int_{\mathbb{R}} d J \rho(J) e^{\beta J \sum_{\alpha=1}^{n} \sigma_{\alpha} s_{\alpha}} \varphi\left(\left\{\sigma_{\alpha}\right\}\right)= \\
=\frac{1}{c} \varphi\left(\left\{s_{\alpha}\right\}\right)+\frac{G(\hat{s})}{c} \int_{\mathbb{R}} d H p_{H}(H) \operatorname{Tr}_{\left\{\sigma_{\alpha}\right\}} \frac{e^{G(\hat{\sigma})+\beta H \sum_{\alpha=1}^{n} \sigma_{\alpha}} \varphi\left(\left\{\sigma_{\alpha}\right\}\right)}{Z_{n G}}- \\
\quad-\int_{\mathbb{R}} d H p_{H}(H) \operatorname{Tr}_{\left\{\sigma_{\alpha}\right\}} e^{G(\hat{\sigma})+\beta H \sum_{\alpha=1}^{n} \sigma_{\alpha}} \int_{\mathbb{R}} d J \rho(J) e^{\beta J \sum_{\alpha=1}^{n} \sigma_{\alpha} s_{\alpha}} \frac{\varphi\left(\left\{\sigma_{\alpha}\right\}\right)}{Z_{n}} .
\end{aligned}
$$

Definindo

$$
T_{n}\left(p_{1}, p_{2},\left\{s_{\alpha}\right\}\right):=\int_{\mathbb{R}} d H p_{H}(H) \operatorname{Tr}_{\left\{\sigma_{\alpha}\right\}} e^{p_{1}\left[G(\hat{\sigma})+\beta H \sum_{\alpha=1}^{n} \sigma_{\alpha}\right]} \int_{\mathbb{R}} d J \rho(J) e^{p_{2} \beta J \sum_{\alpha=1}^{n} \sigma_{\alpha} s_{\alpha}} \varphi\left(\left\{\sigma_{\alpha}\right\}\right),
$$

a equação secular pode ser expressa como

$$
\lambda T_{n}\left(0,1,\left\{s_{\alpha}\right\}\right)=\frac{1}{c} \varphi\left(\left\{s_{\alpha}\right\}\right)+\frac{G(\hat{s})}{c Z_{n G}} T_{n}\left(1,0,\left\{s_{\alpha}\right\}\right)-\frac{1}{Z_{n G}} T_{n}\left(1,1,\left\{s_{\alpha}\right\}\right)
$$

\section{B.8- Prova do teorema de Mottishaw - De Dominicis}

Provar-se-á, aqui, o teorema 3.1. A demonstração deste resultado, introduzido por Mottishaw e de Dominicis, não consta, aparentemente, na literatura.

Teorema 3.1 (Mottishaw - de Dominicis): Na solução réplica-simétrica, com a equação secular dada por (3.44), o espaço dos autovetores $\varphi\left(\left\{\sigma_{\alpha}\right\}\right)$ é varrido pelo conjunto de $2^{n}$ funções de duas variáveis $\varphi_{\left\{\mu_{\alpha}\right\}}^{\prime}\left(\hat{\sigma}, q_{\sigma \mu}\right)$; simbolicamente,

$$
\varphi\left(\left\{\sigma_{\alpha}\right\}\right) \rightarrow \varphi_{\left\{\mu_{\alpha}\right\}}^{\prime}\left(\hat{\sigma}, q_{\sigma \mu}\right),
$$

onde $q_{\sigma \mu}:=\sum_{\alpha=1}^{n} \sigma_{\alpha} \mu_{\alpha}$ e $\left\{\mu_{\alpha}\right\}$ é um conjunto de vetores que rotula o autovetor.

Prova: A equação secular (B.80),

$$
\begin{aligned}
\varphi\left(\left\{s_{\alpha}\right\}\right)= & -G(\hat{s}) \int_{\mathbb{R}} d H p_{H}(H) \operatorname{Tr}_{\left\{\sigma_{\alpha}\right\}} \frac{e^{G(\hat{\sigma})+\beta H \sum_{\alpha=1}^{n} \sigma_{\alpha}} \varphi\left(\left\{\sigma_{\alpha}\right\}\right)}{Z_{n G}}+ \\
& +c \lambda \operatorname{Tr}_{\left\{\sigma_{\alpha}\right\}} \int_{\mathbb{R}} d J \rho(J) e^{\beta J \sum_{\alpha=1}^{n} \sigma_{\alpha} s_{\alpha}} \varphi\left(\left\{\sigma_{\alpha}\right\}\right)+ \\
& +c \int_{\mathbb{R}} d H p_{H}(H) \operatorname{Tr}_{\left\{\sigma_{\alpha}\right\}} e^{G(\hat{\sigma})+\beta H \sum_{\alpha=1}^{n} \sigma_{\alpha}} \int_{\mathbb{R}} d J \rho(J) e^{\beta J \sum_{\alpha=1}^{n} \sigma_{\alpha} s_{\alpha}} \frac{\varphi\left(\left\{\sigma_{\alpha}\right\}\right)}{Z_{n G}}
\end{aligned}
$$

pode ser expressa como 


$$
\varphi\left(\left\{s_{\alpha}\right\}\right)=G(\hat{s}) \operatorname{Tr}_{\left\{\sigma_{\alpha}\right\}} A\left(\left\{\sigma_{\alpha}\right\}\right)+\operatorname{Tr}_{\left\{\sigma_{\alpha}\right\}} \int_{\mathbb{R}} d J \rho(J) B\left(\left\{\sigma_{\alpha}\right\}\right) e^{\beta J \sum_{\alpha=1}^{n} \sigma_{\alpha} s_{\alpha}}
$$

onde $A\left(\left\{\sigma_{\alpha}\right\}\right)$ e $B\left(\left\{\sigma_{\alpha}\right\}\right)$ dependem do conjunto de spins $\left\{\sigma_{\alpha}\right\}$. Denotando por $\vec{\sigma}_{i}$, que é um vetor de $n$ componentes, cada uma das $2^{n}$ configurações englobadas pelo traço, a equação acima assume a forma

$$
\varphi\left(\left\{s_{\alpha}\right\}\right)=\sum_{i=1}^{2^{n}}\left[G(\hat{s}) A\left(\vec{\sigma}_{i}\right)+\int_{\mathbb{R}} d J \rho(J) B\left(\vec{\sigma}_{i}\right) e^{\beta J \sum_{\alpha=1}^{n}\left(\vec{\sigma}_{i}\right)_{\alpha} s_{\alpha}}\right] .
$$

Notando que para cada $i$ corresponde biunivocamente uma configuração $\vec{\sigma}_{i}$ de um vetor (de componentes $\left.\left(\vec{\sigma}_{i}\right)_{\alpha}\right)$, o segundo termo entre colchetes em (B.86) pode ser rotulado por um vetor cujas componentes são $\left\{\mu_{\alpha}\right\}$; em outros termos, $\vec{\sigma}_{i}=\left(\mu_{1}, \cdots, \mu_{n}\right)$, com $\mu_{\alpha} \in\{-1,1\}, \forall \alpha \in\{1, \cdots, n\}$. Logo,

$$
\varphi\left(\left\{s_{\alpha}\right\}\right)=\operatorname{Tr}_{\left\{\mu_{\alpha}\right\}} \underbrace{\left[G(\hat{s}) A\left(\left\{\mu_{\alpha}\right\}\right)+\int_{\mathbb{R}} d J \rho(J) B\left(\left\{\mu_{\alpha}\right\}\right) e^{\beta J q_{s \mu}}\right]}_{\varphi_{\left\{\mu_{\alpha}\right\}}^{\prime}\left(G, q_{s \mu}\right)},
$$

mostrando que $\varphi\left(\left\{s_{\alpha}\right\}\right)$ pode ser representada por uma soma de $2^{n}$ funções de duas variáveis descrita acima. Como a aplicação $\left\{\mu_{\alpha}\right\} \mapsto \sum_{\alpha=1}^{n} \sigma_{\alpha} \mu_{\alpha}$ é injetiva, os $2^{n}$ vetores $\left\{\varphi_{\left\{\mu_{\alpha}\right\}}^{\prime}\left(\hat{s}, q_{s \mu}\right)\right\}$ formam um conjunto linearmente independente, demonstrando que ele varre todo o espaço determinado por $\left\{\varphi\left(\left\{\sigma_{\alpha}\right\}\right)\right\}$ por constituir uma base.

No desenvolvimento da tese, recorrer-se-á a um abuso de notação, omitindo o índice ', e renomeando $\varphi_{\left\{\mu_{\alpha}\right\}}^{\prime}$ por $\varphi_{\left\{\mu_{\alpha}\right\}}$.

\section{B.9- Autovetores longitudinais}

Nesta seção, os autovalores associados a autovetores longitudinais,

$$
\varphi\left(\left\{\sigma_{\alpha}\right\}\right) \rightarrow \varphi(\hat{\sigma})
$$

serão calculados para ilustrar as técnicas envolvidas em sua determinação. O caso mais geral, que será visto mais adiante, é similar a esta situação restrita.

Admite-se que existam campos efetivos que correspondam ao comportamento de

$$
\int_{\mathbb{R}} d H p_{H}(H) e^{p_{1}\left[G(\hat{\sigma})+\beta H \sum_{\alpha=1}^{n} \sigma_{\alpha}\right]} \quad \text { e } \quad \varphi(\hat{\sigma}),
$$

cujas funções distribuição de probabilidades sejam, respectivamente, $P_{1}$ e $P_{L}$. Em outros termos,

$$
\int_{\mathbb{R}} d H p_{H}(H) e^{p_{1}\left[G(\hat{\sigma})+\beta H \sum_{\alpha=1}^{n} \sigma_{\alpha}\right]}=\int_{\mathbb{R}} d h P_{1}(h) e^{\beta h \sum_{\alpha=1}^{n} \sigma_{\alpha}},
$$




$$
\varphi(\hat{\sigma})=\int_{\mathbb{R}} d h P_{L}(h) e^{\beta h \sum_{\alpha=1}^{n} \sigma_{\alpha}}
$$

Através de uma continuação analítica $\mathbb{Z} \ni \hat{\sigma} \rightarrow i y / \beta \in \mathbb{C}, y \in \mathbb{R}$, pode-se mostrar, mediante uma transformada de Fourier, que

$$
P_{\mathbf{1}}(h)=\int_{\mathbb{R}} d y e^{-i y h} \int_{\mathbb{R}} d H p_{H}(H) e^{p_{1}[G(i y / \beta)+i y H]}
$$

e

$$
P_{L}(h)=\int_{\mathbb{R}} d y e^{-i y h} \varphi\left(\frac{i y}{\beta}\right)
$$

Então, (B.81) pode ser escrita como

$$
\begin{aligned}
T_{n}\left(p_{1}, p_{2},\left\{s_{\alpha}\right\}\right)= & \operatorname{Tr}_{\left\{\sigma_{\alpha}\right\}} \int_{\mathbb{R}} d h_{1} P_{1}\left(h_{1}\right) e^{\beta h_{1} \sum_{\alpha=1}^{n} \sigma_{\alpha}} \int_{\mathbb{R}} d J \rho(J) e^{p_{2} \beta J \sum_{\alpha=1}^{n} \sigma_{\alpha} s_{\alpha}} \times \\
& \times \int_{\mathbb{R}} d h_{2} P_{L}\left(h_{2}\right) e^{\beta h_{2} \sum_{\alpha=1}^{n} \sigma_{\alpha}} \\
= & \int_{\mathbb{R}} d h_{1}\left[\int_{\mathbb{R}} d y_{1} e^{-i y_{1} h_{1}} \int_{\mathbb{R}} d H p_{H}(H) e^{p_{1}\left[G\left(i y_{1} / \beta\right)+i y_{1} H\right]}\right] \int_{\mathbb{R}} d h_{2} \times \\
& \times\left[\int_{\mathbb{R}} d y_{2} e^{-i y_{2} h_{2}} \varphi\left(\frac{i y_{2}}{\beta}\right)\right] \int_{\mathbb{R}} d J \rho(J) 2^{n} \prod_{\alpha=1}^{n} \cosh \left[\beta\left(h_{1}+h_{2}\right)+p_{2} \beta J s_{\alpha}\right] .
\end{aligned}
$$

Recorrendo à relação auxiliar (B.184), vem

$$
\begin{aligned}
T_{n}\left(p_{1}, p_{2}, \hat{s}\right)= & \int_{\mathbb{R}} d h_{1}\left[\int_{\mathbb{R}} d y_{1} e^{-i y_{1} h_{1}} \int_{\mathbb{R}} d H p_{H}(H) e^{p_{1}\left[G\left(i y_{1} / \beta\right)+i y_{1} H\right]}\right] \int_{\mathbb{R}} d h_{2} \times \\
& \times\left[\int_{\mathbb{R}} d y_{2} e^{-i y_{2} h_{2}} \varphi\left(\frac{i y_{2}}{\beta}\right)\right] \int_{\mathbb{R}} d J \rho(J) 2^{n}\left[\cosh \left(\beta h_{1}+\beta h_{2}+p_{2} \beta J\right)\right]^{\frac{n}{2}} \times \\
& \times\left[\cosh \left(\beta h_{1}+\beta h_{2}-p_{2} \beta J\right)\right]^{\frac{n}{2}} \exp \left\{\hat{s} \tanh ^{-1}\left[\tanh \left(\beta h_{1}+\beta h_{2}\right) \tanh \left(p_{2} \beta J\right)\right]\right\},
\end{aligned}
$$

que, com a mudança $\left(h_{1}, h_{2}\right) \rightarrow\left(h, h_{3}\right)$, com $h=h_{1}+h_{2}$ e $h_{3}=h_{2}$, toma a forma 
$A P \hat{E} N D I C E B$

93

$$
\begin{aligned}
T_{n}\left(p_{1}, p_{2}, \hat{s}\right)= & \int_{\mathbb{R}} d H p_{H}(H) \int_{\mathbb{R}} d y_{1} \int_{\mathbb{R}} d y_{2} e^{p_{1}\left[G\left(i y_{1} / \beta\right)+i y_{1} H\right]} \varphi\left(\frac{i y_{2}}{\beta}\right) \int_{\mathbb{R}} d h e^{-i y_{1} h} \underbrace{\int_{\mathbb{R}} d h_{3} e^{-i h_{3}\left(y_{2}-y_{1}\right)} \times}_{\delta\left(y_{2}-y_{1}\right)} \\
& \times \int_{\mathbb{R}} d J \rho(J) 2^{n}\left[\cosh \left(\beta h+p_{2} \beta J\right) \cosh \left(\beta h-p_{2} \beta J\right)\right]^{\frac{n}{2}} \times \\
& \times \exp \left\{\hat{s} \tanh ^{-1}\left[\tanh (\beta h) \tanh \left(p_{2} \beta J\right)\right]\right\} \\
= & \int_{\mathbb{R}} d H p_{H}(H) \int_{\mathbb{R}} d y e^{p_{1}[G(i y / \beta)+i y H]} \varphi\left(\frac{i y}{\beta}\right) \int_{\mathbb{R}} d h e^{-i y h} \int_{\mathbb{R}} d J \rho(J) \times \\
& \times\left[4 \cosh \left(\beta h+p_{2} \beta J\right) \cosh \left(\beta h-p_{2} \beta J\right)\right]^{\frac{n}{2}} \exp \left\{\hat{s} \tanh ^{-1}\left[\tanh (\beta h) \tanh \left(p_{2} \beta J\right)\right]\right\} .
\end{aligned}
$$

Impondo uma continuação analítica em $n$ e $\hat{s}$ para considerar a equação acima, (B.96), no ponto $i s / \beta$ (ao invés de $\hat{s}$ ) e no limite $n \rightarrow 0$,

$$
\begin{aligned}
T\left(p_{1}, p_{2}, i s / \beta\right):= & \lim _{n \rightarrow 0} T_{n}\left(p_{1}, p_{2}, i s / \beta\right)=\int_{\mathbb{R}} d H p_{H}(H) \int_{\mathbb{R}} d y e^{p_{1}[G(i y / \beta)+i y H]} \varphi\left(\frac{i y}{\beta}\right) \int_{\mathbb{R}} d h e^{-i y h} \times \\
& \times \int_{\mathbb{R}} d J \rho(J) \exp \left\{\frac{i s}{\beta} \tanh ^{-1}\left[\tanh (\beta h) \tanh \left(p_{2} \beta J\right)\right]\right\} .
\end{aligned}
$$

Na equação secular no limite $n \rightarrow 0$, e usando também o resultado (B.36), vem

$$
\begin{aligned}
\lambda \int_{\mathbb{R}} d y \varphi\left(\frac{i y}{\beta}\right) \int_{\mathbb{R}} d h e^{-i y h} \int_{\mathbb{R}} d J \rho(J) \exp \left\{\frac{i s}{\beta} \tanh ^{-1}[\tanh (\beta h) \tanh (\beta J)]\right\}= \\
=\frac{1}{c} \varphi\left(\frac{i s}{\beta}\right)+\frac{1}{c} G\left(\frac{i s}{\beta}\right) \varphi(0)-e^{-c} \int_{\mathbb{R}} d H p_{H}(H) \int_{\mathbb{R}} d y e^{G(i y / \beta)+i y H} \varphi\left(\frac{i y}{\beta}\right) \int_{\mathbb{R}} d h e^{-i y h} \times \\
\quad \times \int_{\mathbb{R}} d J \rho(J) \exp \left\{\frac{i s}{\beta} \tanh ^{-1}[\tanh (\beta h) \tanh (\beta J)]\right\},
\end{aligned}
$$

C: $\quad$ que, no estado fundamental $\beta \rightarrow \infty$, assume a forma

$$
\begin{aligned}
& \lambda \int_{\mathbb{R}} d y \tilde{\varphi}(i y) \int_{\mathbb{R}} d h e^{-i y h} \int_{\mathbb{R}} d J \rho(J) e^{i s \operatorname{sign}(J h) \min \{|J|,|h|\}}= \\
& =\frac{1}{c} \tilde{\varphi}(i s)+\frac{1}{c} \tilde{G}(i s) \tilde{\varphi}(0)-\int_{\mathbb{R}} d H p_{H}(H) \int_{\mathbb{R}} d y e^{\tilde{G}(i y)-c+i y H} \tilde{\varphi}(i y) \int_{\mathbb{R}} d h e^{-i y h} \times \\
& \quad \times \int_{\mathbb{R}} d J \rho(J) e^{i s \operatorname{sign}(J h) \min \{|J|,|h|\}} .
\end{aligned}
$$


A resolução do problema de autovalores (B.99), que consiste em determinar $\tilde{\varphi}$ com seus respectivos autovalores, é feita através do Ansatz

$$
\tilde{\varphi}(i x)=\tilde{A}+\tilde{B}_{+} e^{i x J_{0}}+\tilde{B}_{-} e^{-i x J_{0}},
$$

que se revelará coerente a posteriori. Ao longo dos cálculos, as equações obtidas no Apêndice B.11 serão invocadas. Adotando as distribuições (B.2), (B.4) e a relação (3.31), a equação secular para os autovetores longitudinais,

$$
\begin{aligned}
\lambda \int_{\mathbb{R}} d y\left[\tilde{A}+\tilde{B}_{+} e^{i y J_{0}}+\tilde{B}_{-} e^{-i y J_{0}}\right] \int_{\mathbb{R}} d h e^{-i y h} e^{i s \operatorname{sign}(h) \min \left\{J_{0},|h|\right\}}= \\
=\frac{1}{c}\left[\tilde{A}+\tilde{B}_{+} e^{i s J_{0}}+\tilde{B}_{-} e^{-i s J_{0}}\right]+\frac{1}{c} \tilde{G}(i s)\left[\tilde{A}+\tilde{B}_{+}+\tilde{B}_{-}\right]- \\
\quad-\int_{\mathbb{R}} d H p_{H}(H) \int_{\mathbb{R}} d y e^{\tilde{G}(i y)-c+i y H}\left[\tilde{A}+\tilde{B}_{+} e^{i y J_{0}}+\tilde{B}_{-} e^{-i y J_{0}}\right] \int_{\mathbb{R}} d h e^{-i y h} e^{i s \operatorname{sign}(h) \min \left\{J_{0},|h|\right\}} \\
=\frac{1}{c}\left[\tilde{A}+\tilde{B}_{+} e^{i s J_{0}}+\tilde{B}_{-} e^{-i s J_{0}}\right]+\frac{1}{c} \tilde{G}(i s)\left[\tilde{A}+\tilde{B}_{+}+\tilde{B}_{-}\right]- \\
\quad-\tilde{A} \int_{\mathbb{R}} d h P(h) e^{i s \operatorname{sign}(h) \min \left\{J_{0},|h|\right\}}-\tilde{B}_{+} \int_{\mathbb{R}} d h P\left(h-J_{0}\right) e^{i s \operatorname{sign}(h) \min \left\{J_{0},|h|\right\}}- \\
-\tilde{B}_{-} \int_{\mathbb{R}} d h P\left(h+J_{0}\right) e^{i s \operatorname{sign}(h) \min \left\{J_{0},|h|\right\}}
\end{aligned}
$$

pode ser expressa, usando (B.49), (B.50) e (B.48), como

$$
\begin{aligned}
\lambda[\tilde{A}+ & \left.\tilde{B}_{+} e^{i s J_{0}}+\tilde{B}_{-} e^{-i s J_{0}}\right]= \\
= & \frac{1}{c}\left[\tilde{A}+\tilde{B}_{+} e^{i s J_{0}}+\tilde{B}_{-} e^{-i s J_{0}}\right]+\frac{1}{c}\left[A+B e^{i s J_{0}}+C e^{-i s J_{0}}\right]\left[\tilde{A}+\tilde{B}_{+}+\tilde{B}_{-}\right]- \\
& -\tilde{A}\left(\frac{A}{c}+\frac{B}{c} e^{i s J_{0}}+\frac{C}{c} e^{-i s J_{0}}\right)-\tilde{B}_{+}\left[a_{-1}+\frac{B+A}{c} e^{i s J_{0}}+\left(\frac{C}{c}-a_{-1}\right) e^{-i s J_{0}}\right]- \\
& -\tilde{B}_{-}\left[a_{1}+\left(\frac{B}{c}-a_{1}\right) e^{i s J_{0}}+\frac{A+C}{c} e^{-i s J_{0}}\right]
\end{aligned}
$$

Da independẽncia linear entre $1, e^{i s J_{0}}$ e $e^{-i s J_{0}}$, o problema de autovalores acima pode ser representado matricialmente por $M_{L} \tilde{\varphi}=\lambda \tilde{\varphi}$, ou

$$
\underbrace{\left(\begin{array}{ccc}
\frac{1}{c} & \frac{A}{c}-a_{-1} & \frac{A}{c}-a_{1} \\
0 & \frac{1}{c}-\frac{A}{c} & a_{1} \\
0 & a_{-1} & \frac{1}{c}-\frac{A}{c}
\end{array}\right)}_{M_{L}}\left(\begin{array}{c}
\tilde{A} \\
\tilde{B}_{+} \\
\tilde{B}_{-}
\end{array}\right)=\lambda\left(\begin{array}{c}
\tilde{A} \\
\tilde{B}_{+} \\
\tilde{B}_{-}
\end{array}\right)
$$

A fim de evitar a solução trivial $\tilde{A}=\tilde{B}_{+}=\tilde{B}_{-}=0$, impõe-se $\operatorname{det}\left(M_{L}-\lambda 1\right)=0$, donde se têm os autovalores longitudinais, 


$$
\begin{gathered}
\lambda_{L 1}=\frac{1}{c}(1-A)+\sqrt{a_{1} a_{-1}}, \\
\lambda_{L 2}=\frac{1}{c}
\end{gathered}
$$

e

$$
\lambda_{L 3}=\frac{1}{c}(1-A)-\sqrt{a_{1} a_{-1}},
$$

que é o menor dos três encontrados.

\section{B.10- Autovetores transversais}

\section{B.10.1- Considerações gerais}

Aqui, pretende-se estudar uma situação geral, onde

$$
\varphi\left(\left\{s_{\alpha}\right\}\right) \rightarrow \varphi_{\left\{\mu_{\alpha}\right\}}\left(\hat{s}, q_{s \mu}\right)=: \varphi\left(\hat{s}, q_{s \mu}\right)
$$

e o autovetor não depende somente de $\hat{s}$, mas também de sua projeção $q_{s \mu}:=\sum_{\alpha=1}^{n} s_{\alpha} \mu_{\alpha}$ ao longo de um vetor $\left(\mu_{1}, \cdots, \mu_{n}\right)$, que caracteriza $\varphi\left(\hat{s}, q_{s \mu}\right)$; o índice. $\left\{\mu_{\alpha}\right\}$ em $\varphi$ é suprimido para não sobrecarregar a notação. O ponto de partida é, novamente, a equação secular (B.82), que será tratada com passos similares ao do caso dos autovetores longitudinais, mas com a introdução do campo efetivo cuja função distribuição de probabilidades é $P_{T}$,

$$
\varphi\left(\hat{s}, q_{s \mu}\right)=\int_{\mathbb{R}} d h_{1} \int_{\mathbb{R}} d h_{2} P_{T}\left(h_{1}, h_{2}\right) e^{\beta h_{1} \hat{s}+\beta h_{2} q_{s \mu}},
$$

e a transformada (inversa) de Fourier a determina em função de $\varphi$,

$$
P_{T}\left(h_{1}, h_{2}\right)=\int_{\mathbb{R}} d y_{1} \int_{\mathbb{R}} d y_{2} e^{-i y_{1} h_{1}-i y_{2} h_{2}} \varphi\left(\frac{i y_{1}}{\beta}, \frac{i y_{2}}{\beta}\right),
$$

onde uma continuação analítica em $\hat{s}$ e $q_{s \mu}$ foi considerada. Então, recorrendo à distribuição $P_{1}$ definida em (B.90) e (B.92), 


$$
\begin{aligned}
T_{n}\left(p_{1}, p_{2},\left\{s_{\alpha}\right\},\left\{\mu_{\alpha}\right\}\right):= & \int_{\mathbb{R}} d H p_{H}(H) \operatorname{Tr}_{\left\{\sigma_{\alpha}\right\}} e^{p_{1}\left[G(\hat{\sigma})+\beta H \sum_{\alpha=1}^{n} \sigma_{\alpha}\right]} \int_{\mathbb{R}} d J \rho(J) e^{p_{2} \beta J \sum_{\alpha=1}^{n} \sigma_{\alpha} s_{\alpha}} \varphi\left(\hat{\sigma}, q_{\sigma \mu}\right) \\
= & \int_{\mathbb{R}} d h_{1} P_{1}\left(h_{1}\right) \iint_{\mathbb{R}} d h_{2} \int_{\mathbb{R}} d h_{3} P_{T}\left(h_{2}, h_{3}\right) \int_{\mathbb{R}} d J \rho(J) \times \\
& \times \prod_{\alpha=1}^{n} \operatorname{Tr}_{\sigma_{\alpha}} e^{\beta\left(h_{1}+h_{2}\right) \sigma_{\alpha}+\beta h_{3} \sigma_{\alpha} \mu_{\alpha}+p_{2} \beta J \sigma_{\alpha} s_{\alpha}} \\
= & \int_{\mathbb{R}} d h \int_{\mathbb{R}} d h_{4} P_{1}\left(h-h_{4}\right) \int_{\mathbb{R}} d h_{3} P_{T}\left(h_{4}, h_{3}\right) \int_{\mathbb{R}} d J \rho(J) \times \\
& \times 2^{n} \prod_{\alpha=1}^{n} \cosh \left(\beta h+\beta h_{3} \mu_{\alpha}+p_{2} \beta J s_{\alpha}\right) \\
= & \int_{\mathbb{R}} d h_{1} \int_{\mathbb{R}} d h_{2} \int_{\mathbb{R}} d y_{1} \int_{\mathbb{R}} d y_{2} \int_{\mathbb{R}} d H p_{H}(H) e^{-i y_{1} h_{1}-i y_{2} h_{2}} e^{p_{1}\left[G\left(i y_{1} / \beta\right)+i y_{1} H\right]} \times \\
& \times \varphi\left(\frac{i y_{1}}{\beta}, \frac{i y_{2}}{\beta}\right) \int_{\mathbb{R}} d J \rho(J) 2^{n} \prod_{\alpha=1}^{n} \cosh \left(\beta h_{1}+\beta h_{2} \mu_{\alpha}+p_{2} \beta J s_{\alpha}\right),
\end{aligned}
$$

onde $\prod_{\alpha=1}^{n} \cosh \left(\beta h_{1}+\beta h_{2} \mu_{\alpha}+p_{2} \beta J s_{\alpha}\right)$ é calculado na seção seguinte (vide (B.184)). Impondo uma continuação analítica em $n, \hat{s}, \hat{\mu}$ e $q_{s \mu}$, e tomando o limite $n \rightarrow 0$ na equação, tem-se

$$
\begin{aligned}
T\left(p_{1}, p_{2}, i \hat{s} / \beta, i \hat{\mu} / \beta, i q_{s \mu} / \beta\right)= & \lim _{n \rightarrow 0} T_{n}\left(p_{1}, p_{2}, i \hat{s} / \beta, i \hat{\mu} / \beta, i q_{s \mu} / \beta\right) \\
= & \int_{\mathbb{R}} d h_{1} \int_{\mathbb{R}} d h_{2} \int_{\mathbb{R}} d y_{1} \int_{\mathbb{R}} d y_{2} \int_{\mathbb{R}} d H p_{H}(H) \int_{\mathbb{R}} d J \rho(J) e^{-i y_{1} h_{1}-i y_{2} h_{2}} \times \\
& \times e^{p_{1}\left[G\left(i y_{1} / \beta\right)+i y_{1} H\right]} \varphi\left(\frac{i y_{1}}{\beta}, \frac{i y_{2}}{\beta}\right) \times \\
& \times\left[\frac{\cosh \left(\beta h_{1}+\beta h_{2}+p_{2} \beta J\right) \cosh \left(\beta h_{1}+\beta h_{2}-p_{2} \beta J\right)}{\cosh \left(\beta h_{1}-\beta h_{2}+p_{2} \beta J\right) \cosh \left(\beta h_{1}-\beta h_{2}-p_{2} \beta J\right)}\right]^{\frac{i \hat{\mu}}{4 \beta}} \times \\
& \times \exp \left\{\frac{i}{2 \beta}\left(\hat{s}+q_{s \mu}\right) \tanh ^{-1}\left[\tanh \left(\beta h_{1}+\beta h_{2}\right) \tanh \left(p_{2} \beta J\right)\right]\right\} \times \\
& \times \exp \left\{\frac{i}{2 \beta}\left(\hat{s}-q_{s \mu}\right) \tanh ^{-1}\left[\tanh \left(\beta h_{1}-\beta h_{2}\right) \tanh \left(p_{2} \beta J\right)\right]\right\}(\mathrm{B} .111)
\end{aligned}
$$

que recupera o caso longitudinal (B.97) se $\varphi\left(\frac{i y_{1}}{\beta}, \frac{i y_{2}}{\beta}\right)=\varphi\left(\frac{i y_{1}}{\beta}\right)$. Com uma mudança de variáveis $h_{1}+h_{2} \rightarrow h_{1}, h_{1}-h_{2} \rightarrow h_{2}, y_{1}+y_{2} \rightarrow y_{1}$ e $y_{1}-y_{2} \rightarrow y_{2}$, e definindo $\chi(x, y):=\varphi(x+y, x-y)$, 


$$
\begin{aligned}
T\left(p_{1}, p_{2}, i \hat{s} / \beta, i \hat{\mu} / \beta, i q_{s \mu} / \beta\right)= & \frac{1}{4} \int_{\mathbb{R}} d h_{1} \int_{\mathbb{R}} d h_{2} \int_{\mathbb{R}} d y_{1} \int_{\mathbb{R}} d y_{2} \int_{\mathbb{R}} d H p_{H}(H) \int_{\mathbb{R}} d J \rho(J) e^{-\frac{i y_{1} h_{1}}{2}-\frac{i y_{2} h_{2}}{2} \times} \\
& \times e^{p_{1}\left[G\left(\frac{i y_{1}+i y_{2}}{2 \beta}\right)+\frac{i y_{1} H}{2}\right]} \chi\left(\frac{i y_{1}}{2 \beta}, \frac{i y_{2}}{2 \beta}\right) \times \\
& \times\left[\frac{\cosh \left(\beta h_{1}+p_{2} \beta J\right) \cosh \left(\beta h_{1}-p_{2} \beta J\right)}{\cosh \left(\beta h_{2}+p_{2} \beta J\right) \cosh \left(\beta h_{2}-p_{2} \beta J\right)}\right]^{\frac{i \beta}{4 \beta}} \times \\
& \times \exp \left\{\frac{i}{2 \beta}\left(\hat{s}+q_{s \mu}\right) \tanh ^{-1}\left[\tanh \left(\beta h_{1}\right) \tanh \left(p_{2} \beta J\right)\right]\right\} \times \\
& \times \exp \left\{\frac{i}{2 \beta}\left(\hat{s}-q_{s \mu}\right) \tanh ^{-1}\left[\tanh \left(\beta h_{2}\right) \tanh \left(p_{2} \beta J\right)\right]\right\} \\
= & \int_{\mathbb{R}} d h_{1} \int_{\mathbb{R}} d h_{2} \int_{\mathbb{R}} d y_{1} \int d y_{\mathbb{R}} \int d H p_{H}(H) \int_{\mathbb{R}} d J \rho(J) e^{-i y_{1} h_{1}-i y_{2} h_{2}} \times \\
& \times e^{p_{1}\left[G\left(\frac{i y_{1}+i y_{2}}{\beta}\right)+i y_{1} H\right]} \chi\left(\frac{i y_{1}}{\beta}, \frac{i y_{2}}{\beta}\right) \times \\
& \times\left[\frac{\cosh \left(\beta h_{1}+p_{2} \beta J\right) \cosh \left(\beta h_{1}-p_{2} \beta J\right)}{\cosh \left(\beta h_{2}+p_{2} \beta J\right) \cosh \left(\beta h_{2}-p_{2} \beta J\right)}\right]^{\frac{i \rho}{4 \beta}} \times \\
& \times \exp \left\{\frac{i}{2 \beta}\left(\hat{s}+q_{s \mu}\right) \tanh { }^{-1}\left[\tanh \left(\beta h_{1}\right) \tanh \left(p_{2} \beta J\right)\right]\right\} \times \\
& \times \exp \left\{\frac{i}{2 \beta}\left(\hat{s}-q_{s \mu}\right) \tanh { }^{-1}\left[\tanh \left(\beta h_{2}\right) \tanh \left(p_{2} \beta J\right)\right]\right\},
\end{aligned}
$$

sendo que na última passagem, recorreu-se à mudança de variáveis $y_{1} \rightarrow 2 y_{1}$ e $y_{2} \rightarrow 2 y_{2}$.

De posse de (B.112), e introduzindo

$$
\eta:=\frac{1}{2}\left(\hat{s}+q_{s \mu}\right) \quad \text { e } \quad \xi:=\frac{1}{2}\left(\hat{s}-q_{s \mu}\right),
$$

a equação secular (B.82) é

$$
\begin{aligned}
& \lambda \int_{\mathbb{R}} d y_{1} \int_{\mathbb{R}} d y_{2} \chi\left(\frac{i y_{1}}{\beta}, \frac{i y_{2}}{\beta}\right) \int_{\mathbb{R}} d h_{1} \int_{\mathbb{R}} d h_{2} e^{-i y_{1} h_{1}-i y_{2} h_{2}} \int_{\mathbb{R}} d J \rho(J) \times \\
& \times\left[\frac{\cosh \left(\beta h_{1}+\beta J\right) \cosh \left(\beta h_{1}-\beta J\right)}{\cosh \left(\beta h_{2}+\beta J\right) \cosh \left(\beta h_{2}-\beta J\right)}\right]^{\frac{i \hat{\beta}}{4 \beta}} e^{\frac{i \eta}{\beta} \tanh ^{-1}\left[\tanh \left(\beta h_{1}\right) \tanh (\beta J)\right]+\frac{i \xi}{\beta} \tanh ^{-1}\left[\tanh \left(\beta h_{2}\right) \tanh (\beta J)\right]}= \\
& =\frac{1}{c} \chi\left(\frac{i \eta}{\beta}, \frac{i \xi}{\beta}\right)+\frac{1}{c} G\left(\frac{i \eta+i \xi}{\beta}\right) \int_{\mathbb{R}} d y_{1} \int_{\mathbb{R}} d y_{2} e^{G\left(\frac{i y_{1}+i y_{2}}{\beta}\right)-c} \chi\left(\frac{i y_{1}}{\beta}, \frac{i y_{2}}{\beta}\right) \int_{\mathbb{R}} d H p_{H}(H) e^{i\left(y_{1}+y_{2}\right) H} \times \\
& \quad \times \int_{\mathbb{R}} d h_{1} \int_{\mathbb{R}} d h_{2} e^{-i y_{1} h_{1}-i y_{2} h_{2}+\frac{i \tilde{\mu}}{\beta} \tanh ^{-1}\left[\tanh \left(\frac{\beta h_{1}+\beta h_{2}}{2}\right) \tanh \left(\frac{\beta h_{1}-\beta h_{2}}{2}\right)\right]}-\int_{\mathbb{R}} d y_{1} \int_{\mathbb{R}} d y_{2} e^{G\left(\frac{i y_{1}+i y_{2}}{\beta}\right)-c} \times \\
& \quad \times \chi\left(\frac{i y_{1}}{\beta}, \frac{i y_{2}}{\beta}\right) \int_{\mathbb{R}} d H p_{H}(H) e^{i\left(y_{1}+y_{2}\right) H} \int_{\mathbb{R}} d h_{1} \int_{\mathbb{R}} d h_{2} e^{-i y_{1} h_{1}-i y_{2} h_{2}} \int_{\mathbb{R}} d J \rho(J) \times \\
& \quad \times\left[\frac{\cosh \left(\beta h_{1}+\beta J\right) \cosh \left(\beta h_{1}-\beta J\right)}{\cosh \left(\beta h_{2}+\beta J\right) \cosh \left(\beta h_{2}-\beta J\right)}\right]^{\frac{i \tilde{i}}{4 \beta}} e^{\frac{i \eta}{\beta} \tanh ^{-1}\left[\tanh \left(\beta h_{1}\right) \tanh (\beta J)\right]+\frac{i \xi}{\beta} \tanh ^{-1}\left[\tanh \left(\beta h_{2}\right) \tanh (\beta J)\right] .}
\end{aligned}
$$




\section{Definindo}

$$
\tilde{\chi}(x, y):=\lim _{\beta \rightarrow \infty} \chi\left(\frac{x}{\beta}, \frac{y}{\beta}\right),
$$

a equação de autovalores no estado fundamental é, usando (B.44) e (B.46),

$$
\begin{aligned}
& \lambda \int_{\mathbb{R}} d y_{1} \int_{\mathbb{R}} d y_{2} \tilde{\chi}\left(i y_{1}, i y_{2}\right) \int_{\mathbb{R}} d h_{1} \int_{\mathbb{R}} d h_{2} e^{-i y_{1} h_{1}-i y_{2} h_{2}} \int_{\mathbb{R}} d J \rho(J) e^{\frac{i \mu}{2}\left[\max \left\{\left|h_{1}\right|,|J|\right\}-\max \left\{\left|h_{2}\right|,|J|\right\}\right]_{\times}} \\
& \times e^{i \eta \operatorname{sign}\left(h_{1} J\right) \min \left\{\left|h_{1}\right|,|J|\right\}+i \xi \operatorname{sign}\left(h_{2} J\right) \min \left\{\left|h_{2}\right|,|J|\right\}}= \\
& =\frac{1}{c} \tilde{\chi}(i \eta, i \xi)+\frac{1}{c} \tilde{G}(i \eta+i \xi) \int_{\mathbb{R}} d y_{1} \int_{\mathbb{R}} d y_{2} e^{\tilde{G}\left(i y_{1}+i y_{2}\right)-c} \tilde{\chi}\left(i y_{1}, i y_{2}\right) \int_{\mathbb{R}} d H p_{H}(H) e^{i\left(y_{1}+y_{2}\right) H} \times \\
& \quad \times \int_{\mathbb{R}} d h_{1} \int_{\mathbb{R}} d h_{2} e^{-i y_{1} h_{1}-i y_{2} h_{2}+\frac{i \mu}{2} \operatorname{sign}\left(h_{1}^{2}-h_{2}^{2}\right) \min \left\{\left|h_{1}+h_{2}\right|,\left|h_{1}-h_{2}\right|\right\}}-\int_{\mathbb{R}} d y_{1} \int_{\mathbb{R}} d y_{2} e^{\tilde{G}\left(i y_{1}+i y_{2}\right)-c} \times \\
& \quad \times \tilde{\chi}\left(i y_{1}, i y_{2}\right) \int_{\mathbb{R}} d H p_{H}(H) e^{i\left(y_{1}+y_{2}\right) H} \int_{\mathbb{R}} d h_{1} \int_{\mathbb{R}} d h_{2} e^{-i y_{1} h_{1}-i y_{2} h_{2}} \int_{\mathbb{R}} d J \rho(J) \times \\
& \quad \times e^{\frac{i \dot{\mu}}{2}\left[\max \left\{\left|h_{1}\right|,|J|\right\}-\max \left\{\left|h_{2}\right|,|J|\right\}\right]} e^{i \eta \operatorname{sign}\left(h_{1} J\right) \min \left\{\left|h_{1}\right|,|J|\right\}+i \xi \operatorname{sign}\left(h_{2} J\right) \min \left\{\left|h_{2}\right|,|J|\right\}} .
\end{aligned}
$$

A equação secular acima, para autovetores transversais, será analisada em duas situações distintas, $\hat{\mu}=0$ e $\hat{\mu} \neq 0$. Ao longo dos cálculos, as equaçōes obtidas no Apêndice B.11 serão invocadas.

\section{B.10.2- Caso $\hat{\mu}=0$}

Impondo $\hat{\mu}=0$ na equação (B.116), tem-se

$$
\begin{aligned}
\lambda \int_{\mathbb{R}} d h_{1} \int_{\mathbb{R}} d h_{2} \int_{\mathbb{R}} d y_{1} \int_{\mathbb{R}} d y_{2} e^{-i y_{1} h_{1}-i y_{2} h_{2}} \tilde{\chi}\left(i y_{1}, i y_{2}\right) e^{i \eta \operatorname{sign}\left(h_{1}\right) \min \left\{\left|h_{1}\right|, J_{0}\right\}} e^{i \xi \operatorname{sign}\left(h_{2}\right) \min \left\{\left|h_{2}\right|, J_{0}\right\}}= \\
=\frac{1}{c} \tilde{\chi}(i \eta, i \xi)+\frac{\tilde{G}(i \eta+i \xi)}{c} \tilde{\chi}(0,0)-\int_{\mathbb{R}} d h_{1} \int_{\mathbb{R}} d h_{2} \int_{\mathbb{R}} d y_{1} \int_{\mathbb{R}} d y_{2} e^{-i y_{1} h_{1}-i y_{2} h_{2}} e^{\tilde{G}\left(i y_{1}+i y_{2}\right)-c} \times \\
\times\left[\frac{1}{2} e^{i y_{1} H+i y_{2} H}+\frac{1}{2} e^{-i y_{1} H-i y_{2} H}\right] \tilde{\chi}\left(i y_{1}, i y_{2}\right) e^{i \eta \operatorname{sign}\left(h_{1}\right) \min \left\{\left|h_{1}\right|, J_{0}\right\}} e^{i \xi \operatorname{sign}\left(h_{2}\right) \min \left\{\left|h_{2}\right|, J_{0}\right\}},
\end{aligned}
$$

que é a equação a ser resolvida. O Ansatz

$$
\begin{aligned}
\tilde{\chi}(i \eta, i \xi)= & \tilde{A}+\tilde{B}_{+} e^{i \eta J_{0}}+\tilde{B}_{-} e^{-i \eta J_{0}}+\tilde{C}_{+} e^{i \xi J_{0}}+\tilde{C}_{-} e^{-i \xi J_{0}}+ \\
& +\tilde{D}_{++} e^{i \eta J_{0}+i \xi J_{0}}+\tilde{D}_{+-} e^{i \eta J_{0}-i \xi J_{0}}+\tilde{D}_{-+} e^{-i \eta J_{0}+i \xi J_{0}}+\tilde{D}_{--} e^{-i \eta J_{0}-i \xi J_{0}}
\end{aligned}
$$

é uma possível solução de (B.117), o que será confirmado a posteriori. Introduzindo-o na equação secular (B.117), e calculando separadamente cada termo, tem-se

Primeiro termo (primeiro membro): 
$k$

$A P \hat{E} N D I C E B$

Usando as notações

$$
\tilde{\chi}(i \eta, i \xi)=\sum_{u, v=0, \pm 1} K(u, v) e^{i u \eta J_{0}+i v \xi J_{0}}
$$

com $K(0,0):=\tilde{A}, K(1,0):=\tilde{B}$, e assim por diante (vide (B.118)), e

$$
\ddagger x \ddagger^{J}:=\operatorname{sign}(x) \min \left\{|x|, J_{0}\right\} \quad \text { e } \quad \ddagger x \ddagger:=\operatorname{sign}(x) \min \{|x|, 1\},
$$

o primeiro termo do primeiro membro pode ser escrito como

$$
\begin{aligned}
\lambda \int_{\mathbb{R}} d h_{1} \int_{\mathbb{R}} d h_{2} \int_{\mathbb{R}} d y_{1} \int_{\mathbb{R}} d y_{2} e^{-i y_{1} h_{1}-i y_{2} h_{2}} \sum_{u, v=0, \pm 1} K(u, v) e^{i u y_{1} J_{0}+i v y_{2} J_{0}} e^{i \eta \ddagger h_{1} \ddagger^{J}+i \xi \ddagger h_{2} \ddagger^{J}}= \\
=\sum_{u, v=0, \pm 1} K(u, v) \int_{\mathbb{R}} d h_{1} \int_{\mathbb{R}} d h_{2} \delta\left(h_{1}-u J_{0}\right) \delta\left(h_{2}-v J_{0}\right) e^{i \eta \ddagger h_{1} \ddagger^{J}+i \xi \ddagger h_{2} \ddagger^{J}} \\
=\sum_{u, v=0, \pm 1} K(u, v) e^{i \eta J_{0} \ddagger u \ddagger+i \xi J_{0} \ddagger v \ddagger} \\
=\quad \lambda\left(\tilde{A}+\tilde{B}_{+} e^{i \eta J_{0}}+\tilde{B}_{-} e^{-i \eta J_{0}}+\tilde{C}_{+} e^{i \xi J_{0}}+\tilde{C}_{-} e^{-i \xi J_{0}}+\right. \\
\left.\quad+\tilde{D}_{++} e^{i \eta J_{0}+i \xi J_{0}}+\tilde{D}_{+-} e^{i \eta J_{0}-i \xi J_{0}}+\tilde{D}_{-+} e^{-i \eta J_{0}+i \xi J_{0}}+\tilde{D}_{--} e^{-i \eta J_{0}-i \xi J_{0}}\right) .
\end{aligned}
$$

$\underline{\text { Primeiro termo (segundo membro): }}$

$$
\begin{aligned}
& \frac{1}{c}\left(\tilde{A}+\tilde{B}_{+} e^{i \eta J_{0}}+\tilde{B}_{-} e^{-i \eta J_{0}}+\tilde{C}_{+} e^{i \xi J_{0}}+\tilde{C}_{-} e^{-i \xi J_{0}}+\right. \\
& \left.\quad+\tilde{D}_{++} e^{i \eta J_{0}+i \xi J_{0}}+\tilde{D}_{+-} e^{i \eta J_{0}-i \xi J_{0}}+\tilde{D}_{-+} e^{-i \eta J_{0}+i \xi J_{0}}+\tilde{D}_{--} e^{-i \eta J_{0}-i \xi J_{0}}\right) .
\end{aligned}
$$

\section{Segundo termo (segundo membro):}

$$
\frac{1}{c}\left(A+B e^{i \eta J_{0}+i \xi J_{0}}+C e^{-i \eta J_{0}-i \xi J_{0}}\right)\left(\tilde{A}+\tilde{B}_{+}+\tilde{B}_{-}+\tilde{C}_{+}+\tilde{C}_{-}+\tilde{D}_{++}+\tilde{D}_{+-}+\tilde{D}_{-+}+\tilde{D}_{--}\right)
$$

\section{Terceiro termo (segundo membro):}

Recorrendo às notações (B.119) e (B.120), e de (B.49), 


$$
\begin{aligned}
& \int_{\mathbb{R}} d h_{1} \int_{\mathbb{R}} d h_{2} \int_{\mathbb{R}} d y_{1} \int_{\mathbb{R}} d y_{2} e^{-i y_{1} h_{1}-i y_{2} h_{2}} \exp \left[A+B e^{i y_{1} J_{0}+i y_{2} J_{0}}+C e^{-i y_{1} J_{0}-i y_{2} J_{0}}-c\right] \times \\
& \times\left[\frac{1}{2} e^{i y_{1} H+i y_{2} H}+\frac{1}{2} e^{-i y_{1} H-i y_{2} H}\right] \sum_{u, v=0, \pm 1} K(u, v) e^{i u y_{1} J_{0}+i v y_{2} J_{0}} e^{i \eta \ddagger h_{1} \ddagger^{J}+i \xi \ddagger h_{2} \ddagger^{J}}= \\
& =\frac{e^{A-c}}{2} \sum_{u, v=0, \pm \mathbf{1}} K(u, v) \int_{\mathbb{R}} d h_{1} \int_{\mathbb{R}} d h_{2} \int_{\mathbb{R}} d y_{1} \int_{\mathbb{R}} d y_{2} e^{-i y_{1} h_{1}-i y_{2} h_{2}} \sum_{r=0}^{\infty} \frac{B^{r}}{r !} e^{i r\left(y_{1}+y_{2}\right) J_{0}} \times \\
& \times \sum_{s=0}^{\infty} \frac{C^{s}}{s !} e^{-i s\left(y_{1}+y_{2}\right) J_{0}}\left[e^{i\left(y_{1}+y_{2}\right) m J_{0}}+e^{-i\left(y_{1}+y_{2}\right) m J_{0}}\right] e^{i u y_{1} J_{0}+i v y_{2} J_{0}} e^{i \eta \ddagger h_{1} \ddagger^{J}+i \xi \ddagger h_{2} \ddagger^{J}} \\
& =\frac{e^{A-c}}{2} \sum_{u, v=0, \pm 1} K(u, v) \sum_{r=0}^{\infty} \sum_{s=0}^{\infty} \frac{B^{r} C^{s}}{r ! s !} \int_{\mathbb{R}} d h_{1} \int_{\mathbb{R}} d h_{2} e^{i \eta \ddagger h_{1} \ddagger^{J}+i \xi \ddagger h_{2} \ddagger^{J}} \times \\
& \times\left[\delta\left(h_{1}-r J_{0}+s J_{0}-m J_{0}-u J_{0}\right) \delta\left(h_{2}-r J_{0}+s J_{0}-m J_{0}-v J_{0}\right)+\right. \\
& \left.+\delta\left(h_{1}-r J_{0}+s J_{0}+m J_{0}-u J_{0}\right) \delta\left(h_{2}-r J_{0}+s J_{0}+m J_{0}-v J_{0}\right)\right] \\
& =\frac{e^{A-c}}{2} \sum_{u, v=0, \pm 1} K(u, v) \underbrace{\sum_{r=0}^{\infty} \sum_{s=0}^{\infty} \frac{B^{r} C^{s}}{r ! s !} e^{i \eta J_{0} \ddagger r-s+m+u \ddagger+i \xi J_{0} \ddagger r-s+m+v \ddagger}}+ \\
& \text { (i) } \\
& +\frac{e^{A-c}}{2} \sum_{u, v=0, \pm 1} K(u, v) \underbrace{\sum_{r=0}^{\infty} \sum_{s=0}^{\infty} \frac{B^{r} C^{s}}{r ! s !} e^{i \eta J_{0} \ddagger r-s-m+u \ddagger+i \xi J_{0} \ddagger r-s-m+v \ddagger}} . \\
& \text { (ii) }
\end{aligned}
$$

As séries (i) e (ii) serão desenvolvidas separadamente. Em $(i)$, se denotar $k:=r-s+m+u$ e recorrer a (B.60), a série é decomposta em

$$
\begin{aligned}
\sum_{r=0}^{\infty} & \sum_{s=0}^{\infty} \frac{B^{r} C^{s}}{r ! s !} e^{i \eta J_{0} \ddagger r-s+m+u \ddagger+i \xi J_{0} \ddagger r-s+m+v \ddagger}= \\
= & \sum_{k \geq m+u} \sum_{s=0}^{\infty} \frac{B^{k+s-m-u} C^{s}}{(k+s-m-u) ! s !} e^{i \eta J_{0} \ddagger k \ddagger+i \xi J_{0} \ddagger k-u+v \ddagger}+ \\
& +\sum_{k<m+u} \sum_{r=0}^{\infty} \frac{B^{r} C^{r+m+u-k}}{r !(r+m+u-k) !} e^{i \eta J_{0} \ddagger k \ddagger+i \xi J_{0} \ddagger k-u+v \ddagger} \\
= & \sum_{k=m+u}^{\infty}\left(\frac{B}{C}\right)^{\frac{k-m-u}{2}} I_{k-m-u}(2 \sqrt{B C}) e^{i \eta J_{0} \ddagger k \ddagger+i \xi J_{0} \ddagger k-u+v \ddagger}+ \\
& +\sum_{k=-\infty}^{m+u-1}\left(\frac{B}{C}\right)^{\frac{k-m-u}{2}} I_{k-m-u}(2 \sqrt{B C}) e^{i \eta J_{0} \ddagger k \ddagger+i \xi J_{0} \ddagger k-u+v \ddagger} \\
= & \sum_{k \in \mathbb{Z}}\left(\frac{B}{C}\right)^{\frac{k-m-u}{2}} .
\end{aligned}
$$

Analogamente, a série (ii) é escrita como (é suficiente substituir $m$ por $-m$ em (B.125))

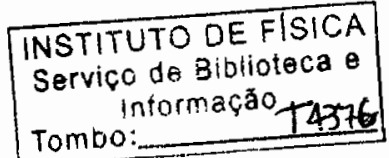




$$
\sum_{k \in \mathbb{Z}}\left(\frac{B}{C}\right)^{\frac{k+m-u}{2}} I_{k+m-u}(2 \sqrt{B C}) e^{i \eta J_{0} \ddagger k \ddagger+i \xi J_{0} \ddagger k-u+v \ddagger} .
$$

Invocando as relações (B.67) e (B.50), e reunindo (B.125) e (B.126), o terceiro termo do segundo membro é, finalmente,

$$
\begin{aligned}
& \sum_{u, v=0, \pm 1} K(u, v) \sum_{k \in \mathbb{Z}} a_{k-u} e^{i \eta J_{0} \ddagger k \ddagger+i \xi J_{0} \ddagger k-u+v \ddagger}= \\
& =\sum_{u, v=0, \pm 1} K(u, v)\left[a_{-u} e^{i \xi J_{0} \ddagger-u+v \ddagger}+\sum_{k=1}^{\infty} a_{k-u} e^{i \eta J_{0}+i \xi J_{0} \ddagger k-u+v \ddagger}+\sum_{k=-\infty}^{-1} a_{k-u} e^{-i \eta J_{0}+i \xi J_{0} \ddagger k-u+v \ddagger}\right],
\end{aligned}
$$

que é uma representação compacta de

$$
\begin{array}{rl}
\sum_{u, v=0, \pm 1} & K(u, v) \sum_{k \in \mathbb{Z}} a_{k-u} e^{i \eta J_{0} \ddagger k \ddagger+i \xi J_{0} \ddagger k-u+v \ddagger}= \\
= & \underbrace{K(0,0)}_{\tilde{A}}\left[\frac{A}{c}+\frac{B}{c} e^{i \eta J_{0}+i \xi J_{0}}+\frac{C}{c} e^{-i \eta J_{0}-i \xi J_{0}}\right]+\underbrace{K(1,0)}_{\tilde{B}_{+}}\left[a_{-1} e^{-i \xi J_{0}}+\frac{A}{c} e^{i \eta J_{0}}+\frac{B}{c} e^{i \eta J_{0}+i \xi J_{0}}+\right. \\
& \left.+\left(\frac{C}{c}-a_{-1}\right) e^{-i \eta J_{0}-i \xi J_{0}}\right]+\underbrace{K(-1,0)}_{\tilde{B}_{-}}\left[a_{1} e^{i \xi J_{0}}+\left(\frac{B}{c}-a_{1}\right) e^{i \eta J_{0}+i \xi J_{0}}+\frac{A}{c} e^{-i \eta J_{0}}+\right. \\
& \left.+\frac{C}{c} e^{-i \eta J_{0}-i \xi J_{0}}\right]+\underbrace{K(0,1)}_{\tilde{C}_{+}}\left[\frac{A}{c} e^{i \xi J_{0}}+\frac{B}{c} e^{i \eta J_{0}+i \xi J_{0}}+a_{-1} e^{-i \eta J_{0}}+\left(\frac{C}{c}-a_{-1}\right) e^{-i \eta J_{0}-i \xi J_{0}}\right]+ \\
& +\underbrace{K(0,-1)}_{\tilde{C}-}\left[\frac{A}{c} e^{-i \xi J_{0}}+a_{1} e^{i \eta J_{0}}+\left(\frac{B}{c}-a_{1}\right) e^{i \eta J_{0}+i \xi J_{0}}+\frac{C}{c} e^{-i \eta J_{0}-i \xi J_{0}}\right]+\underbrace{K(1,1)}_{\tilde{D}++} \times \\
& \times\left[a_{-1}+\left(\frac{A+B}{c}\right) e^{i \eta J_{0}+i \xi J_{0}}+\left(\frac{C}{c}-a_{-1}\right) e^{-i \eta J_{0}-i \xi J_{0}}\right]+\underbrace{K(1,-1)}_{\tilde{D}_{+-}} \times \\
& \times\left[a_{-1} e^{-i \xi J_{0}}+\frac{A}{c} e^{i \eta J_{0}-i \xi J_{0}}+a_{1} e^{i \eta J_{0}}+\left(\frac{B}{c}-a_{1}\right) e^{i \eta J_{0}+i \xi J_{0}}+\left(\frac{C}{c}-a_{-1}\right) e^{-i \eta J_{0}-i \xi J_{0}}\right]+ \\
& +\underbrace{K(-1,1)}_{\tilde{D}-+}\left[a_{1} e^{i \xi J_{0}}+\left(\frac{B}{c}-a_{1}\right) e^{i \eta J_{0}+i \xi J_{0}}+\frac{A}{c} e^{-i \eta J_{0}+i \xi J_{0}}+a_{-1} e^{-i \eta J_{0}}+\right. \\
+ & \left.\left(\frac{C}{c}-a_{-1}\right) e^{-i \eta J_{0}-i \xi J_{0}}\right]+\underbrace{K(-1,-1)}_{\tilde{D}--}\left[a_{1}+\left(\frac{B}{c}-a_{1}\right) e^{i \eta J_{0}+i \xi J_{0}}+\left(\frac{A+C}{c}\right) e^{-i \eta J_{0}-i \xi J_{0}}\right] .
\end{array}
$$

Reunindo (B.121), (B.122), (B.123) e (B.128) na equação de autovalores, e pela independência linear entre os vetores $1, e^{i \eta J_{0}}, e^{-i \eta J_{0}}, e^{i \xi J_{0}}, e^{-i \xi J_{0}}, e^{i \eta J_{0}+i \xi J_{0}}, e^{i \eta J_{0}-i \xi J_{0}}, e^{-i \eta J_{0}+i \xi J_{0}}$ e $e^{-i \eta J_{0}-i \xi J_{0}}$, o problema pode ser reescrito matricialmente como $M_{T 0} \tilde{\chi}=\lambda \tilde{\chi}$, onde 


$$
\tilde{\chi}^{T}=\left(\tilde{A}, \tilde{B}_{+}, \tilde{B}_{-}, \tilde{C}_{+}, \tilde{C}_{-}, \tilde{D}_{++}, \tilde{D}_{+-}, \tilde{D}_{-+}, \tilde{D}_{--}\right)
$$

e

$$
M_{T 0}:=\left(\begin{array}{ccccccccc}
\frac{1}{c} & \frac{A}{c} & \frac{A}{c} & \frac{A}{c} & \frac{A}{c} & \frac{A}{c}-a_{-1} & \frac{A}{c} & \frac{A}{c} & \frac{A}{c}-a_{1} \\
0 & \frac{1}{c}-\frac{A}{c} & 0 & 0 & -a_{1} & 0 & -a_{1} & 0 & 0 \\
0 & 0 & \frac{1}{c}-\frac{A}{c} & -a_{-1} & 0 & 0 & 0 & -a_{-1} & 0 \\
0 & 0 & -a_{1} & \frac{1}{c}-\frac{A}{c} & 0 & 0 & 0 & -a_{1} & 0 \\
0 & -a_{-1} & 0 & 0 & \frac{1}{c}-\frac{A}{c} & 0 & -a_{-1} & 0 & 0 \\
0 & 0 & a_{1} & 0 & a_{1} & \frac{1}{c}-\frac{A}{c} & a_{1} & a_{1} & a_{1} \\
0 & 0 & 0 & 0 & 0 & 0 & \frac{1}{c}-\frac{A}{c} & 0 & 0 \\
0 & 0 & 0 & 0 & 0 & 0 & 0 & \frac{1}{c}-\frac{A}{c} & 0 \\
0 & a_{-1} & 0 & a_{-1} & 0 & a_{-1} & a_{-1} & a_{-1} & \frac{1}{c}-\frac{A}{c}
\end{array}\right)
$$

Os autovalares da matriz $M_{T 0}$ são

$$
\begin{gathered}
\lambda_{T_{\hat{\mu}=0} 1}=\frac{1}{c} \quad \text { (não-degenerado) }, \\
\lambda_{T_{\hat{\mu}=0} 2}=\frac{1}{c}(1-A) \quad(\text { degenerescência dupla) }, \\
\lambda_{T_{\hat{A}=0} 3}=\frac{1}{c}(1-A)+\sqrt{a_{1} a_{-1}} \quad \text { (degenerescência tripla) },
\end{gathered}
$$

e

$$
\lambda_{T_{\hat{\mu}=04}}=\frac{1}{c}(1-A)-\sqrt{a_{1} a_{-1}} \quad \text { (degenerescência tripla) } .
$$

Notar que três dos quatro tipos de autovalores encontrados acima já foram obtidos no caso de autovetores longitudinais. $O$ menor deles continua sendo $\lambda_{L 3}=\lambda_{T_{\mu=0} 4}$. Na esperança de conseguir novos autovalores, tratar-se-á o caso mais geral, $\hat{\mu} \neq 0$.

\section{B.10.3- Caso $\hat{\mu} \neq 0$}

O autovetor, aqui, deve conter o parâmetro $\hat{\mu}$, ausente nos casos anteriores. O Ansatz

$$
\tilde{\chi}_{\hat{\mu}}(i \eta, i \xi)=\tilde{\chi}(i \eta, i \xi)=\sum_{\theta \in \mathbb{Z}} \sum_{u, v=0, \pm 1} K_{\theta}(u, v) e^{i u \eta J_{0}+i v \xi J_{0}+\frac{i \hat{\mu} \theta J_{0}}{2}}
$$

é uma possível solução da equação secular 


$$
\begin{aligned}
& \lambda \int_{\mathbb{R}} d y_{1} \int_{\mathbb{R}} d y_{2} \tilde{\chi}\left(i y_{1}, i y_{2}\right) \int_{\mathbb{R}} d h_{1} \int_{\mathbb{R}} d h_{2} e^{-i y_{1} h_{1}-i y_{2} h_{2}} \int_{\mathbb{R}} d J \rho(J) e^{\frac{i \tilde{\mu}}{2}\left[\max \left\{\left|h_{1}\right|,|J|\right\}-\max \left\{\left|h_{2}\right|,|J|\right\} \mid\right.} \times \\
& \times e^{i \eta \operatorname{sign}\left(h_{1} J\right) \min \left\{\left|h_{1}\right|,|J|\right\}+i \xi \operatorname{sign}\left(h_{2} J\right) \min \left\{\left|h_{2}\right|,|J|\right\}}= \\
& =\frac{1}{c} \tilde{\chi}(i \eta, i \xi)+\frac{1}{c} \tilde{G}(i \eta+i \xi) \int_{\mathbb{R}} d y_{1} \int_{\mathbb{R}} d y_{2} e^{\bar{G}\left(i y_{1}+i y_{2}\right)-c} \tilde{\chi}\left(i y_{1}, i y_{2}\right) \int_{\mathbb{R}} d H p_{H}(H) e^{i\left(y_{1}+y_{2}\right) H} \times \\
& \quad \times \int_{\mathbb{R}} d h_{1} \int_{\mathbb{R}} d h_{2} e^{-i y_{1} h_{1}-i y_{2} h_{2}+\frac{i \hat{\mu}}{2} \operatorname{sign}\left(h_{1}^{2}-h_{2}^{2}\right) \min \left\{\left|h_{1}+h_{2}\right|,\left|h_{1}-h_{2}\right|\right\}}-\int_{\mathbb{R}} d y_{1} \int_{\mathbb{R}} d y_{2} e^{\tilde{G}\left(i y_{1}+i y_{2}\right)-c} \times \\
& \quad \times \tilde{\chi}\left(i y_{1}, i y_{2}\right) \int_{\mathbb{R}} d H p_{H}(H) e^{i\left(y_{1}+y_{2}\right) H} \int_{\mathbb{R}} d h_{1} \int_{\mathbb{R}} d h_{2} e^{-i y_{1} h_{1}-i y_{2} h_{2}} \int_{\mathbb{R}} d J \rho(J) \times \\
& \quad \times e^{\frac{i \tilde{\mu}}{2}\left[\max \left\{\left|h_{1}\right|,|J|\right\}-\max \left\{\left|h_{2}\right|,|J|\right\} \mid\right.} e^{i \eta \operatorname{sign}\left(h_{1} J\right) \min \left\{\left|h_{1}\right|,|J|\right\}+i \xi \operatorname{sign}\left(h_{2} J\right) \min \left\{\left|h_{2}\right|,|J|\right\}} .
\end{aligned}
$$

Novamente, os cálculos são efetuados termo a termo, considerando (B.3), (B.2) e a notação (B.120). Primeiro termo (primeiro membro):

$$
\begin{aligned}
& \lambda \int_{\mathbb{R}} d y_{1} \int_{\mathbb{R}} d y_{2} \sum_{\theta \in \mathbb{Z}} \sum_{u, v=0, \pm 1} K_{\theta}(u, v) e^{i u y_{1} J_{0}+i v y_{2} J_{0}+\frac{i \dot{\beta} \theta J_{0}}{2}} \int_{\mathbb{R}} d h_{1} \int_{\mathbb{R}} d h_{2} e^{-i y_{1} h_{1}-i y_{2} h_{2}} \times \\
& \times e^{\frac{i \mu}{2}\left[\max \left\{\left|h_{1}\right|, J_{0}\right\}-\max \left\{\left|h_{2}\right|, J_{0}\right\}\right]} e^{i \eta \ddagger h_{1} \ddagger^{J}+i \xi \ddagger h_{2} \ddagger^{J}}= \\
& =\lambda \sum_{\theta \in \mathbb{Z}} \sum_{u, v=0, \pm 1} K_{\theta}(u, v) \int_{\mathbb{R}} d h_{1} \int_{\mathbb{R}} d h_{2} \delta\left(h_{1}-u J_{0}\right) \delta\left(h_{2}-v J_{0}\right) e^{\frac{i \dot{\mu} \theta J_{0}}{2}} \times \\
& \times e^{\frac{i \xi}{2}\left[\max \left\{\left|h_{1}\right|, J_{0}\right\}-\max \left\{\left|h_{2}\right|, J_{0}\right\}\right]} e^{i \eta \ddagger h_{1} \ddagger^{J}+i \xi \ddagger h_{2} \ddagger^{J}} \\
& =\lambda \sum_{\theta \in \mathbb{Z}} \sum_{u, v=0, \pm 1} K_{\theta}(u, v) e^{\frac{i \hat{\mu} \theta J_{0}}{2}} e^{\frac{i \hat{\mu} J_{0}}{2}[\max \{|u|, 1\}-\max \{|v|, 1\}]} e^{i \eta J_{0} \ddagger u \ddagger+i \xi J_{0} \ddagger v \ddagger} \\
& =\lambda \sum_{\theta \in \mathbb{Z}} e^{\frac{i \mu \hat{\theta} J_{0}}{2}} \sum_{u, v=0, \pm 1} K_{\theta}(u, v) e^{i u \eta J_{0}+i v \xi J_{0}} \\
& =\lambda \tilde{\chi}(i \eta, i \xi) \text {, }
\end{aligned}
$$

que é uma forma condensada de se escrever

$$
\begin{aligned}
& \lambda \sum_{\theta \in \mathbb{Z}} e^{\frac{i \mu \theta J_{0}}{2}}\left(\tilde{A}_{\theta}+\tilde{B}_{\theta+} e^{i \eta J_{0}}+\tilde{B}_{\theta-} e^{-i \eta J_{0}}+\tilde{C}_{\theta+} e^{i \xi J_{0}}+\tilde{C}_{\theta-} e^{-i \xi J_{0}}+\right. \\
& \left.\quad+\tilde{D}_{\theta++} e^{i \eta J_{0}+i \xi J_{0}}+\tilde{D}_{\theta+-} e^{i \eta J_{0}-i \xi J_{0}}+\tilde{D}_{\theta-+} e^{-i \eta J_{0}+i \xi J_{0}}+\tilde{D}_{\theta--} e^{-i \eta J_{0}-i \xi J_{0}}\right) .
\end{aligned}
$$

Primeiro termo (segundo membro):

$$
\frac{1}{c} \sum_{\theta \in \mathbb{Z}} \sum_{u, v=0, \pm 1} K_{\theta}(u, v) e^{i u \eta J_{0}+i v \xi J_{0}+\frac{i \boldsymbol{\mu} \theta J_{0}}{2}}=\frac{1}{c} \tilde{\chi}(i \eta, i \xi)
$$


Segundo termo (segundo membro):

De (B.49) e (B.135),

$$
\begin{aligned}
& \frac{1}{c} \tilde{G}(i \eta+i \xi) \int_{\mathbb{R}} d y_{1} \int_{\mathbb{R}} d y_{2} e^{\bar{G}\left(i y_{1}+i y_{2}\right)-c} \tilde{\chi}\left(i y_{1}, i y_{2}\right) \int_{\mathbb{R}} d H p_{H}(H) e^{i\left(y_{1}+y_{2}\right) H} \times \\
& \times \int_{\mathbb{R}} d h_{1} \int_{\mathbb{R}} d h_{2} e^{-i y_{1} h_{1}-i y_{2} h_{2}+\frac{i}{2} \operatorname{sign}\left(h_{1}^{2}-h_{2}^{2}\right) \min \left\{\left|h_{1}+h_{2}\right|,\left|h_{1}-h_{2}\right|\right\}}= \\
& =\frac{e^{A-c}}{c} \tilde{G}(i \eta+i \xi) \int_{\mathbb{R}} d y_{1} \int_{\mathbb{R}} d y_{2} \sum_{r=0}^{\infty} \frac{B^{r}}{r !} e^{i r\left(y_{1}+y_{2}\right) J_{0}} \sum_{s=0}^{\infty} \frac{C^{s}}{s !} e^{-i s\left(y_{1}+y_{2}\right) J_{0}} \sum_{\theta \in \mathbb{Z}} \sum_{u, v=0, \pm 1} K_{\theta}(u, v) \times \\
& \times e^{i u y_{1} J_{0}+i v y_{2} J_{0}+\frac{i \alpha \theta J_{0}}{2}}\left[\frac{e^{i m\left(y_{1}+y_{2}\right) J_{0}}+e^{-i m\left(y_{1}+y_{2}\right) J_{0}}}{2}\right] \int_{\mathbb{R}} d h_{1} \int_{\mathbb{R}} d h_{2} e^{-i y_{1} h_{1}-i y_{2} h_{2}} \times \\
& \times e^{\frac{i \mu}{2} \operatorname{sign}\left(h_{1}^{2}-h_{2}^{2}\right) \min \left\{\left|h_{1}+h_{2}\right|,\left|h_{1}-h_{2}\right|\right\}} \\
& =\frac{e^{A-c}}{2 c} \tilde{G}(i \eta+i \xi) \sum_{\theta \in \mathbb{Z}} \sum_{u, v=0, \pm 1} K_{\theta}(u, v) e^{\frac{i \mu \theta J_{0}}{2}} \int_{\mathbb{R}} d h_{1} \int_{\mathbb{R}} d h_{2} e^{\frac{i \hat{\mu}}{2} \operatorname{sign}\left(h_{1}^{2}-h_{2}^{2}\right) \min \left\{\left|h_{1}+h_{2}\right|,\left|h_{1}-h_{2}\right|\right\}} \times \\
& \times \sum_{r=0}^{\infty} \sum_{s=0}^{\infty} \frac{B^{r} C^{s}}{r ! s !}\left[\delta\left(h_{1}-r J_{0}+s J_{0}-u J_{0}-m J_{0}\right) \delta\left(h_{2}-r J_{0}+s J_{0}-v J_{0}-m J_{0}\right)+\right. \\
& \left.+\delta\left(h_{1}-r J_{0}+s J_{0}-u J_{0}+m J_{0}\right) \delta\left(h_{2}-r J_{0}+s J_{0}-v J_{0}+m J_{0}\right)\right] \\
& =\frac{e^{A-c}}{2 c} \tilde{G}(i \eta+i \xi) \sum_{\theta \in \mathbb{Z}} \sum_{u, v=0, \pm 1} K_{\theta}(u, v) e^{\frac{i \hat{\mu} \theta J_{0}}{2}} \times \\
& \times\{\underbrace{\sum_{r=0}^{\infty} \sum_{s=0}^{\infty} \frac{B^{r} C^{s}}{r ! s !} e^{\frac{i \hat{\mu}}{2} \operatorname{sign}[(2 r-2 s+2 m+u+v)(u-v)] \min \{|2 r-2 s+2 m+u+v|,|u-v|\}}}_{(i)}+ \\
& +\underbrace{\sum_{r=0}^{\infty} \sum_{s=0}^{\infty} \frac{B^{r} C^{s}}{r ! s !} e^{\frac{i \mu}{2} \operatorname{sign}[(2 r-2 s-2 m+u+v)(u-v)] \min \{|2 r-2 s-2 m+u+v|,|u-v|\}}}_{(i i)}\} .
\end{aligned}
$$

As séries $(i)$ e $(i i)$ serão tratadas separadamente. Denotando $k:=r-s+m$, a série $(i)$ pode ser desenvolvida como

$$
\begin{aligned}
\sum_{r=0}^{\infty} \sum_{s=0}^{\infty} \frac{B^{r} C^{s}}{r ! s !} e^{\frac{i \beta}{2} \operatorname{sign}[(2 r-2 s+2 m+u+v)(u-v)] \min \{|2 r-2 s+2 m+u+v|,|u-v|\}}= \\
=\sum_{k \geq m} \sum_{s=0}^{\infty} \frac{B^{k+s-m} C^{s}}{(k+s-m) ! s !} e^{\frac{i \hat{\mu}}{2} \operatorname{sign}[(2 k+u+v)(u-v)] \min \{|2 k+u+v|,|u-v|\}}+ \\
\quad+\sum_{k<m} \sum_{r=0}^{\infty} \frac{B^{r} C^{r-k+m}}{r !(r-k+m) !} e^{\frac{i \tilde{\mu}}{2} \operatorname{sign}[(2 k+u+v)(u-v)] \min \{|2 k+u+v|,|u-v|\}}
\end{aligned}
$$


que, com (B.60),

$$
\begin{aligned}
& \sum_{k=m}^{\infty}\left(\frac{B}{C}\right)^{\frac{k-m}{2}} I_{k-m}(2 \sqrt{B C}) e^{\frac{i \mu}{2} \operatorname{sign}[(2 k+u+v)(u-v)] \min \{|2 k+u+v|,|u-v|\}}+ \\
& +\sum_{k=-\infty}^{m-1}\left(\frac{B}{C}\right)^{\frac{k-m}{2}} I_{k-m}(2 \sqrt{B C}) e^{\frac{i \hat{\mu}}{2} \operatorname{sign}[(2 k+u+v)(u-v)] \min \{|2 k+u+v|,|u-v|\}}= \\
& =\sum_{k \in \mathbb{Z}}\left(\frac{B}{C}\right)^{\frac{k-m}{2}} I_{k-m}(2 \sqrt{B C}) e^{\frac{i \hat{\mu}}{2} \operatorname{sign}[(2 k+u+v)(u-v)] \min \{|2 k+u+v|,|u-v|\}} .
\end{aligned}
$$

Analogamente, a série (ii) é (é suficiente substituir $m$ de (B.142) por $-m$ )

$$
\sum_{k \in \mathbb{Z}}\left(\frac{B}{C}\right)^{\frac{k+m}{2}} I_{k+m}(2 \sqrt{B C}) e^{\frac{i \mu}{2} \operatorname{sign}[(2 k+u+v)(u-v)] \min \{|2 k+u+v|,|u-v|\}}
$$

observando que aqui, inicialmente, foi tomado como $k:=r-s-m$.

Reunindo (B.142) e (B.143) em (B.140), e por (B.67), tem-se

$$
\frac{1}{c} \tilde{G}(i \eta+i \xi) \sum_{\theta \in \mathbb{Z}} \sum_{u, v=0, \pm 1} K_{\theta}(u, v) e^{\frac{i \dot{\mu} \theta J_{0}}{2}} \sum_{k \in \mathbb{Z}} a_{k} e^{\frac{i \hat{\mu}}{2} \operatorname{sign}[(2 k+u+v)(u-v)] \min \{|2 k+u+v|,|u-v|\}}
$$

ou

$$
\begin{aligned}
& \frac{1}{c}\left(A+B e^{i \eta J_{0}+i \xi J_{0}}+C e^{-i \eta J_{0}-i \xi J_{0}}\right) \sum_{\theta \in \mathbb{Z}} e^{\frac{i \mu \theta J_{0}}{2}}\left\{\tilde{A}_{\theta}+\left[\left(\frac{A+B}{c}\right) e^{\frac{i \hat{\alpha} J_{0}}{2}}+\frac{C}{c} e^{-\frac{i \hat{\mu} J_{0}}{2}}\right] \tilde{B}_{\theta+}+\right. \\
& +\left[\left(\frac{A+C}{c}\right) e^{\frac{i \beta J_{0}}{2}}+\frac{B}{c} e^{-\frac{i \hat{\alpha} J_{0}}{2}}\right] \tilde{B}_{\theta-}+\left[\left(\frac{A+B}{c}\right) e^{-\frac{i \alpha J_{0}}{2}}+\frac{C}{c} e^{\frac{i a J_{0}}{2}}\right] \tilde{C}_{\theta+}+ \\
& +\left[\left(\frac{A+C}{c}\right) e^{-\frac{i \hat{a} J_{0}}{2}}+\frac{B}{c} e^{\frac{i \hat{\mu} J_{0}}{2}}\right] \tilde{C}_{\theta-}+\tilde{D}_{\theta++}+\left(\frac{A}{c}+\frac{B}{c} e^{i \hat{\mu} J_{0}}+\frac{C}{c} e^{-i \hat{\mu} J_{0}}\right) \tilde{D}_{\theta+-}+ \\
& \left.+\left(\frac{A}{c}+\frac{B}{c} e^{-i \hat{\mu} J_{0}}+\frac{C}{c} e^{i \hat{\mu} J_{0}}\right) \tilde{D}_{\theta-+}+\tilde{D}_{\theta--}\right\}= \\
& =\frac{1}{c}\left(A+B e^{i \eta J_{0}+i \xi J_{0}}+C e^{-i \eta J_{0}-i \xi J_{0}}\right) \sum_{\theta \in \mathbb{Z}} e^{\frac{i \hat{\theta} \theta J_{0}}{2}}\left\{e ^ { \frac { i \hat { \mu } J _ { 0 } } { 2 } } \left[\left(\frac{A+B}{c}\right) \tilde{B}_{\theta+}+\left(\frac{A+C}{c}\right) \tilde{B}_{\theta-}+\right.\right. \\
& \left.\frac{C}{c} \tilde{C}_{\theta+}+\frac{B}{c} \tilde{C}_{\theta-}\right]+e^{-\frac{i a J_{0}}{2}}\left[\frac{C}{c} \tilde{B}_{\theta+}+\frac{B}{c} \tilde{B}_{\theta-}+\left(\frac{A+B}{c}\right) \tilde{C}_{\theta+}+\left(\frac{A+C}{c}\right) \tilde{C}_{\theta-}\right]+ \\
& +e^{i \hat{\mu} J_{0}}\left(\frac{B}{c} \tilde{D}_{\theta+-} \frac{C}{c} \tilde{D}_{\theta-+}\right)+e^{-i \hat{\mu} J_{0}}\left(\frac{C}{c} \tilde{D}_{\theta+-} \frac{B}{c} \tilde{D}_{\theta-+}\right)+\left(\tilde{A}_{\theta}+\tilde{D}_{\theta++}+\frac{A}{c} \tilde{D}_{\theta+-}+\right. \\
& \left.\left.+\frac{A}{c} \tilde{D}_{\theta-+}+\tilde{D}_{\theta--}\right)\right\} .
\end{aligned}
$$


A fim de simplificar os cálculos posteriormente, defina

$$
\begin{aligned}
t_{2}(\hat{\mu}):= & \sum_{\theta \in \mathbb{Z}} e^{\frac{i \hat{\mu} \theta J_{0}}{2}}\left\{e^{\frac{i \hat{\alpha} J_{0}}{2}}\left[\left(\frac{A+B}{c}\right) \tilde{B}_{\theta+}+\left(\frac{A+C}{c}\right) \tilde{B}_{\theta-}+\frac{C}{c} \tilde{C}_{\theta+}+\frac{B}{c} \tilde{C}_{\theta-}\right]+e^{-\frac{i \hat{\mu} J_{0}}{2}}\left[\frac{C}{c} \tilde{B}_{\theta+}+\right.\right. \\
& \left.+\frac{B}{c} \tilde{B}_{\theta-}+\left(\frac{A+B}{c}\right) \tilde{C}_{\theta+}+\left(\frac{A+C}{c}\right) \tilde{C}_{\theta-}\right]+e^{i \hat{\mu} J_{0}}\left(\frac{B}{c} \tilde{D}_{\theta+-} \frac{C}{c} \tilde{D}_{\theta-+}\right)+ \\
& \left.+e^{-i \hat{\mu} J_{0}}\left(\frac{C}{c} \tilde{D}_{\theta+-} \frac{B}{c} \tilde{D}_{\theta-+}\right)+\left(\tilde{A}_{\theta}+\tilde{D}_{\theta++}+\frac{A}{c} \tilde{D}_{\theta+-}+\frac{A}{c} \tilde{D}_{\theta-+}+\tilde{D}_{\theta--}\right)\right\}
\end{aligned}
$$

que é a parte que multiplica $\tilde{G}(i \eta+i \xi) / c$ no segundo termo do segundo membro em (B.136). Esta função pode ser reescrita como

$$
\begin{aligned}
t_{2}(\hat{\mu})= & \sum_{\theta \in \mathbb{Z}} e^{\frac{i \hat{\mu} \theta J_{0}}{2}}\left\{\left[\left(\frac{A+B}{c}\right) \tilde{B}_{(\theta-1)+}+\left(\frac{A+C}{c}\right) \tilde{B}_{(\theta-1)-}+\frac{C}{c} \tilde{C}_{(\theta-1)+}+\frac{B}{c} \tilde{C}_{(\theta-1)-}\right]+\right. \\
& +\left[\frac{C}{c} \tilde{B}_{(\theta+1)+}+\frac{B}{c} \tilde{B}_{(\theta+1)-}+\left(\frac{A+B}{c}\right) \tilde{C}_{(\theta+1)+}+\left(\frac{A+C}{c}\right) \tilde{C}_{(\theta+1)-}\right]+ \\
& +\left(\frac{B}{c} \tilde{D}_{(\theta-2)+-} \frac{C}{c} \tilde{D}_{(\theta-2)-+}\right)+\left(\frac{C}{c} \tilde{D}_{(\theta+2)+-} \frac{B}{c} \tilde{D}_{(\theta+2)-+}\right)+ \\
& \left.+\left(\tilde{A}_{\theta}+\tilde{D}_{\theta++}+\frac{A}{c} \tilde{D}_{\theta+-}+\frac{A}{c} \tilde{D}_{\theta-+}+\tilde{D}_{\theta--}\right)\right\}
\end{aligned}
$$

Terceiro termo (segundo membro):

De (B.49) e (B.135), 
5

$$
\begin{aligned}
& \int_{\mathbb{R}} d y_{1} \int_{\mathbb{R}} d y_{2} e^{\bar{G}\left(i y_{1}+i y_{2}\right)-c} \tilde{\chi}\left(i y_{1}, i y_{2}\right)\left[\frac{e^{i\left(y_{1}+y_{2}\right) m J_{0}}+e^{-i\left(y_{1}+y_{2}\right) m J_{0}}}{2}\right] \int_{\mathbb{R}} d h_{1} \int_{\mathbb{R}} d h_{2} \times \\
& \times e^{-i y_{1} h_{1}-i y_{2} h_{2}} e^{\frac{i \tilde{\mu}}{2}\left[\max \left\{\left|h_{1}\right|, J_{0}\right\}-\max \left\{\left|h_{2}\right|, J_{0}\right\}\right]} e^{i \eta \ddagger h_{1} \ddagger^{J}+i \xi \ddagger h_{2} \ddagger^{J}}= \\
& =\frac{e^{A-c}}{2} \sum_{\theta \in \mathbb{Z}} \sum_{u, v=0, \pm 1} K_{\theta}(u, v) e^{\frac{i \hat{\theta} \theta J_{0}}{2}} \int_{\mathbb{R}} d h_{1} \int_{\mathbb{R}} d h_{2} \int_{\mathbb{R}} d y_{1} \int_{\mathbb{R}} d y_{2} e^{i u y_{1} J_{0}+i v y_{2} J_{0}} \sum_{r=0}^{\infty} \times \\
& \times \frac{B^{r}}{r !} e^{i r\left(y_{1}+y_{2}\right) J_{0}} \sum_{s=0}^{\infty} \frac{C^{s}}{s !} e^{-i s\left(y_{1}+y_{2}\right) J_{0}}\left[e^{i\left(y_{1}+y_{2}\right) m J_{0}}+e^{-i\left(y_{1}+y_{2}\right) m J_{0}}\right] e^{-i y_{1} h_{1}-i y_{2} h_{2}} \times \\
& \times e^{\left.\frac{i \xi}{2}\left[\max \left\{\mid h_{1}\right\}, J_{0}\right\}-\max \left\{\left|h_{2}\right|, J_{0}\right\}\right]} e^{i \eta \ddagger h_{1} \ddagger^{J}+i \xi \ddagger h_{2} \ddagger^{J}} \\
& =\frac{e^{A-c}}{2} \sum_{\theta \in \mathbb{Z}} \sum_{u, v=0, \pm 1} K_{\theta}(u, v) e^{\frac{i \hat{\beta} \theta J_{0}}{2}} \int_{\mathbb{R}} d h_{1} \int_{\mathbb{R}} d h_{2} e^{\frac{i \hat{\mu}}{2}\left[\max \left\{\left|h_{1}\right|, J_{0}\right\}-\max \left\{\left|h_{2}\right|, J_{0}\right\}\right]} \times \\
& \times \sum_{r=0}^{\infty} \sum_{s=0}^{\infty} \frac{B^{r} C^{s}}{r ! s !}\left[\delta\left(h_{1}-r J_{0}+s J_{0}-u J_{0}-m J_{0}\right) \delta\left(h_{2}-r J_{0}+s J_{0}-v J_{0}-m J_{0}\right)+\right. \\
& \left.+\delta\left(h_{1}-r J_{0}+s J_{0}-u J_{0}+m J_{0}\right) \delta\left(h_{2}-r J_{0}+s J_{0}-v J_{0}+m J_{0}\right)\right] e^{i \eta \ddagger h_{1} \ddagger^{J}+i \xi \ddagger h_{2} \ddagger^{J}} \\
& =\frac{e^{A-c}}{2} \sum_{\theta \in \mathbb{Z}} \sum_{u, v=0, \pm 1} K_{\theta}(u, v) e^{\frac{i \mu \theta J_{0}}{2}} \times \\
& \times\{\underbrace{\sum_{r=0}^{\infty} \sum_{s=0}^{\infty} \frac{B^{r} C^{s}}{r ! s !} e^{\frac{i \hat{\mu} J_{0}}{2} \max \{|r-s+u+m|, 1\}-\max \{|r-s+v+m|, 1\}} e^{i \eta J_{0} \ddagger r-s+u+m \ddagger+i \xi J_{0} \ddagger r-s+v+m \ddagger}}_{(i)}+ \\
& +\underbrace{\sum_{r=0}^{\infty} \sum_{s=0}^{\infty} \frac{B^{r} C^{s}}{r ! s !} e^{\frac{i \hat{\mu} J_{0}}{2} \max \{|r-s+u-m|, 1\}-\max \{|r-s+v-m|, 1\}} e^{i \eta J_{0} \ddagger r-s+u-m \ddagger+i \xi J_{0} \ddagger r-s+v-m \ddagger}}_{(i i)} .
\end{aligned}
$$

Defina $k:=r-s+u+m$ a fim de efetuar a série $(i)$,

$$
\begin{aligned}
\sum_{r=0}^{\infty} \sum_{s=0}^{\infty} \frac{B^{r} C^{s}}{r ! s !} e^{\frac{i \mu J_{0}}{2} \max \{|r-s+u+m|, 1\}-\max \{|r-s+v+m|, 1\}} e^{i \eta J_{0} \ddagger r-s+u+m \ddagger+i \xi J_{0} \ddagger r-s+v+m \ddagger}= \\
=\sum_{k \geq u+m} \sum_{s=0}^{\infty} \frac{B^{k+s-u-m} C^{s}}{(k+s-u-m) ! s !} e^{\frac{i \tilde{\mu} J_{0}}{2}[\max \{|k|, 1\}-\max \{|k-u+v|, 1\}]} e^{i \eta J_{0} \ddagger k \ddagger+i \xi J_{0} \ddagger k-u+v \ddagger}+ \\
\quad+\sum_{k<u+m} \sum_{\tau=0}^{\infty} \frac{B^{r} C^{r+m+u-k}}{r !(r+m+u-k) !} e^{\frac{i \hat{a} J_{0}}{2}[\max \{|k|, 1\}-\max \{|k-u+v|, 1\}]} e^{i \eta J_{0} \ddagger k \ddagger+i \xi J_{0} \ddagger k-u+v \ddagger},
\end{aligned}
$$

que, com (B.60), pode ser escrita como 


$$
\sum_{k \in \mathbb{Z}}\left(\frac{B}{C}\right)^{\frac{k-u-m}{2}} I_{k-u-m}(2 \sqrt{B C}) e^{\frac{i \hat{\mu} J_{0}}{2}[\max \{|k|, 1\}-\max \{|k-u+v|, 1\}]} e^{i \eta J_{0} \ddagger k \ddagger+i \xi J_{0} \ddagger k-u+v \ddagger} .
$$

Analogamente, a série (ii) é

$$
\sum_{k \in \mathbb{Z}}\left(\frac{B}{C}\right)^{\frac{k-u+m}{2}} I_{k-u+m}(2 \sqrt{B C}) e^{\frac{i \tilde{\mu} J_{0}}{2}[\max \{|k|, 1\}-\max \{|k-u+v|, 1\}]} e^{i \eta J_{0} \ddagger k \ddagger+i \xi J_{0} \ddagger k-u+v \ddagger} .
$$

Reunindo (B.150) e (B.151) em (B.148), e de (B.67), o terceiro termo do segundo membro de (B.136) é

$$
\sum_{\theta \in \mathbb{Z}} \sum_{u, v=0, \pm 1} K_{\theta}(u, v) e^{\frac{i \hat{\mu} \theta J_{0}}{2}} \sum_{k \in \mathbb{Z}} a_{k-u} e^{\frac{i \hat{\mu} J_{0}}{2}[\max \{|k|, 1\}-\max \{|k-u+v|, 1\}]} e^{i \eta J_{0} \ddagger k \ddagger+i \xi J_{0} \ddagger k-u+v \ddagger}
$$

ou

$$
\begin{aligned}
& \sum_{\theta \in \mathbb{Z}} \sum_{u, v=0, \pm 1} K_{\theta}(u, v) e^{\frac{i \hat{\mu} \theta J_{0}}{2}}\left\{a_{-u} e^{\frac{i \hat{\mu} J_{0}}{2}[1-\max \{|u-v|, 1\}]} e^{i \xi J_{0} \ddagger-u+v \ddagger}+\sum_{k=1}^{\infty} a_{k-u} e^{\frac{i \hat{\mu} J_{0}}{2}[k-\max \{|k-u+v|, 1\}]} \times\right. \\
& \left.\times e^{i \eta J_{0}+i \xi J_{0} \ddagger k-u+v \ddagger}+\sum_{k=-\infty}^{-1} a_{k-u} e^{\frac{i \hat{\mu} J_{0}}{2}[|k|-\max \{|k-u+v|, 1\}]} e^{-i \eta J_{0}+i \xi J_{0} \ddagger k-u+v \ddagger}\right\}
\end{aligned}
$$

que é uma forma mais condensada de se escrever o terceiro termo do segundo membro de (B.136), denotado doravante por $t_{3}(\eta, \xi, \hat{\mu})$,

$$
\begin{aligned}
t_{3}(\eta, \xi, \hat{\mu}):= & \sum_{\theta \in \mathbb{Z}} e^{\frac{i \mu \theta J_{0}}{2}}\left\{\left(\frac{A}{c} \tilde{A}_{\theta}+a_{-1} \tilde{D}_{\theta++}+a_{1} D_{\theta--}\right)+e^{i \eta J_{0}}\left(\frac{A}{c} \tilde{B}_{\theta+}+a_{1} \tilde{C}_{\theta-}\right)+\right. \\
& +e^{-i \eta J_{0}}\left(\frac{A}{c} \tilde{B}_{\theta-}+a_{-1} \tilde{C}_{\theta+}\right)+e^{i \xi J_{0}}\left(a_{1} \tilde{B}_{\theta-}+\frac{A}{c} \tilde{C}_{\theta+}\right)+e^{-i \xi J_{0}}\left(a_{-1} \tilde{B}_{\theta+}+\right. \\
& \left.+\frac{A}{c} \tilde{C}_{\theta-}\right)+e^{i \eta J_{0}+i \xi J_{0}}\left[\frac{B}{c} \tilde{A}_{\theta}+\left(\frac{A+B}{c}\right) \tilde{D}_{\theta++}+\left(\frac{B}{c}-a_{1}\right) \tilde{D}_{\theta--}\right]+ \\
& +e^{i \eta J_{0}-i \xi J_{0}} \frac{A}{c} \tilde{D}_{\theta+-}+e^{-i \eta J_{0}+i \xi J_{0}} \frac{A}{c} \tilde{D}_{\theta-+}+e^{-i \eta J_{0}-i \xi J_{0}}\left[\frac{C}{c} \tilde{A}_{\theta}+\left(\frac{C}{c}-a_{-1}\right) \tilde{D}_{\theta++}+\right. \\
& \left.+\left(\frac{A+C}{c}\right) \tilde{D}_{\theta--}\right]+e^{\frac{i \hat{\mu} J_{0}}{2}+i \eta J_{0}} a_{1} \tilde{D}_{\theta+-}+e^{\frac{i \xi J_{0}}{2}-i \eta J_{0}} a_{-1} \tilde{D}_{\theta-+}+ \\
& +e^{-\frac{i \hat{\mu} J_{0}}{2}+i \xi J_{0}} a_{1} \tilde{D}_{\theta-+}+e^{-\frac{i \hat{\mu} J_{0}}{2}-i \xi J_{0}} a_{-1} \tilde{D}_{\theta+-}+e^{\frac{i \hat{\mu} J_{0}}{2}+i \eta J_{0}+i \xi J_{0}}\left[\frac{B}{c} \tilde{B}_{\theta+}+\right. \\
& \left.+\left(\frac{B}{c}-a_{1}\right) \tilde{C}_{\theta-}\right]+e^{-\frac{i \hat{\mu} J_{0}}{2}+i \eta J_{0}+i \xi J_{0}}\left[\left(\frac{B}{c}-a_{1}\right) \tilde{B}_{\theta-}+\frac{B}{c} \tilde{C}_{\theta+}\right]+ \\
& +e^{\frac{i \hat{\mu} J_{0}}{2}-i \eta J_{0}-i \xi J_{0}}\left[\frac{C}{c} \tilde{B}_{\theta-}+\left(\frac{C}{c}-a_{-1}\right) \tilde{C}_{\theta+}\right]+e^{-\frac{i \hat{\mu} J_{0}}{2}-i \eta J_{0}-i \xi J_{0}}\left[\left(\frac{C}{c}-a_{-1}\right) \tilde{B}_{\theta+}+\right. \\
& \left.+\frac{C}{c} \tilde{C}_{\theta-}\right]+e^{i \hat{\mu} J_{0}+i \eta J_{0}+i \xi J_{0}}\left(\frac{B}{c}-a_{1}\right) \tilde{D}_{\theta+-}+e^{-i \hat{\mu} J_{0}+i \eta J_{0}+i \xi J_{0}}\left(\frac{B}{c}-a_{1}\right) \tilde{D}_{\theta-+}+ \\
& \left.+e^{i \hat{\mu} J_{0}-i \eta J_{0}-i \xi J_{0}}\left(\frac{C}{c}-a_{-1}\right) \tilde{D}_{\theta-+}+e^{-i \hat{\mu} J_{0}-i \eta J_{0}-i \xi J_{0}}\left(\frac{C}{c}-a_{-1}\right) \tilde{D}_{\theta+-}\right\}, \quad(\mathrm{B} .154)
\end{aligned}
$$


ou

$$
\begin{aligned}
t_{3}(\eta, \xi, \hat{\mu}):= & \sum_{\theta \in \mathbb{Z}} e^{\frac{i \hat{\theta} \theta J_{0}}{2}}\left\{\left[\frac{A}{c} \tilde{A}_{\theta}+a_{-1} \tilde{D}_{\theta++}+a_{1} D_{\theta--}\right]+e^{i \eta J_{0}}\left[\frac{A}{c} \tilde{B}_{\theta+}+a_{1} \tilde{C}_{\theta-}+a_{1} \tilde{D}_{(\theta-1)+-}\right]+\right. \\
& +e^{-i \eta J_{0}}\left[\frac{A}{c} \tilde{B}_{\theta-}+a_{-1} \tilde{C}_{\theta+}+a_{-1} \tilde{D}_{(\theta-1)-+}\right]+e^{i \xi J_{0}}\left[a_{1} \tilde{B}_{\theta-}+\frac{A}{c} \tilde{C}_{\theta+}+a_{1} \tilde{D}_{(\theta+1)-+}\right]+ \\
& +e^{-i \xi J_{0}}\left[a_{-1} \tilde{B}_{\theta+}+\frac{A}{c} \tilde{C}_{\theta-}+a_{-1} \tilde{D}_{(\theta+1)+-}\right]+e^{i \eta J_{0}+i \xi J_{0}}\left[\frac{B}{c} \tilde{A}_{\theta}+\left(\frac{A+B}{c}\right) \tilde{D}_{\theta++}+\right. \\
& +\left(\frac{B}{c}-a_{1}\right) \tilde{D}_{\theta--}+\frac{B}{c} \tilde{B}_{(\theta-1)+}+\left(\frac{B}{c}-a_{1}\right) \tilde{C}_{(\theta-1)-}+\left(\frac{B}{c}-a_{1}\right) \tilde{B}_{(\theta+1)-}+ \\
& \left.+\frac{B}{c} \tilde{C}_{(\theta+1)+}+\left(\frac{B}{c}-a_{1}\right) \tilde{D}_{(\theta-2)+-}+\left(\frac{B}{c}-a_{1}\right) \tilde{D}_{(\theta+2)-+}\right]+e^{i \eta J_{0}-i \xi J_{0}}\left[\frac{A}{c} \tilde{D}_{\theta+-}\right]+ \\
& +e^{-i \eta J_{0}+i \xi J_{0}}\left[\frac{A}{c} \tilde{D}_{\theta-+}\right]+e^{-i \eta J_{0}-i \xi J_{0}}\left[\frac{C}{c} \tilde{A}_{\theta}+\left(\frac{C}{c}-a_{-1}\right) \tilde{D}_{\theta++}+\left(\frac{A+C}{c}\right) \tilde{D}_{\theta--+}+\right. \\
& +\frac{C}{c} \tilde{B}_{(\theta-1)-}+\left(\frac{C}{c}-a_{-1}\right) \tilde{C}_{(\theta-1)+}+\left(\frac{C}{c}-a_{-1}\right) \tilde{B}_{(\theta+1)+}+\frac{C}{c} \tilde{C}_{(\theta+1)-}+ \\
& \left.\left.+\left(\frac{C}{c}-a_{-1}\right) \tilde{D}_{(\theta-2)-+}+\left(\frac{C}{c}-a_{-1}\right) \tilde{D}_{(\theta+2)+-}\right]\right\} .
\end{aligned}
$$

Os resultados (B.138),(B.139), (B.145) (com (B.147)) e (B.155) constituem os termos da equação secular. Pela independência linear entre $1, e^{i \eta J_{0}}, e^{-i \eta J_{0}}, e^{i \xi J_{0}}, e^{-i \xi J_{0}}, e^{i \eta J_{0}+i \xi J_{0}}, e^{i \eta J_{0}-i \xi J_{0}}, e^{-i \eta J_{0}+i \xi J_{0}}$ e $e^{-i \eta J_{0}-i \xi J_{0}}$, nove equações podem ser, inicialmente, escritas,

$$
\begin{aligned}
& -\frac{A}{c} t_{2}(\hat{\mu})+\sum_{\theta \in \mathbb{Z}} e^{\frac{i \mu \theta J_{0}}{2}}\left\{\left(\lambda-\frac{1}{c}\right) \tilde{A}_{\theta}+\frac{A}{c} \tilde{A}_{\theta}+a_{-1} \tilde{D}_{\theta++}+a_{1} \tilde{D}_{\theta--}\right\}=0 \\
& \sum_{\theta \in \mathbb{Z}} e^{\frac{i \mu \theta J_{0}}{2}}\left\{\left(\lambda-\frac{1}{c}\right) \tilde{B}_{\theta+}+\frac{A}{c} \tilde{B}_{\theta+}+a_{1} \tilde{C}_{\theta-}+a_{1} \tilde{D}_{(\theta-1)+-}\right\}=0 \\
& \sum_{\theta \in \mathbb{Z}} e^{\frac{i \hat{\mu} \theta J_{0}}{2}}\left\{\left(\lambda-\frac{1}{c}\right) \tilde{B}_{\theta-}+\frac{A}{c} \tilde{B}_{\theta-}+a_{-1} \tilde{C}_{\theta+}+a_{-1} \tilde{D}_{(\theta-1)-+}\right\}=0 \\
& \quad \sum_{\theta \in \mathbb{Z}} e^{\frac{i \mu \theta J_{0}}{2}}\left\{\left(\lambda-\frac{1}{c}\right) \tilde{C}_{\theta+}+a_{1} \tilde{B}_{\theta-}+\frac{A}{c} \tilde{C}_{\theta+}+a_{1} \tilde{D}_{(\theta+1)-+}\right\}=0 \\
& \quad \sum_{\theta \in \mathbb{Z}} e^{\frac{i \tilde{\mu} \theta J_{0}}{2}}\left\{\left(\lambda-\frac{1}{c}\right) \tilde{C}_{\theta-}+a_{-1} \tilde{B}_{\theta+}+\frac{A}{c} \tilde{C}_{\theta-}+a_{-1} \tilde{D}_{(\theta+1)+-}\right\}=0 \\
& -\frac{B}{c} t_{2}(\hat{\mu})+\sum_{\theta \in \mathbb{Z}} e^{\frac{i \alpha \theta J_{0}}{2}}\left\{\left(\lambda-\frac{1}{c}\right) \tilde{D}_{\theta++}+\frac{B}{c} \tilde{A}_{\theta}+\left(\frac{A+B}{c}\right) \tilde{D}_{\theta++}+\left(\frac{B}{c}-a_{1}\right) \tilde{D}_{\theta--}\right. \\
& +\frac{B}{c} \tilde{B}_{(\theta-1)+}+\left(\frac{B}{c}-a_{1}\right) \tilde{C}_{(\theta-1)-}+\left(\frac{B}{c}-a_{1}\right) \tilde{B}_{(\theta+1)-}+\frac{B}{c} \tilde{C}_{(\theta+1)+}+ \\
& \left.+\left(\frac{B}{c}-a_{1}\right) \tilde{D}_{(\theta-2)+-}+\left(\frac{B}{c}-a_{1}\right) \tilde{D}_{(\theta+2)-+}\right\}=0
\end{aligned}
$$




$$
\begin{aligned}
& \sum_{\theta \in \mathbb{Z}} e^{\frac{i \hat{\mu} \theta J_{0}}{2}}\left\{\left(\lambda-\frac{1}{c}\right) \tilde{D}_{\theta+-}+\frac{A}{c} \tilde{D}_{\theta+-}\right\}=0, \\
& \sum_{\theta \in \mathbb{Z}} e^{\frac{i \hat{\mu} \theta J_{\theta}}{2}}\left\{\left(\lambda-\frac{1}{c}\right) \tilde{D}_{\theta-+}+\frac{A}{c} \tilde{D}_{\theta-+}\right\}=0
\end{aligned}
$$

e

$$
\begin{aligned}
& -\frac{C}{c} t_{2}(\hat{\mu})+\sum_{\theta \in \mathbb{Z}} e^{\frac{i \hat{\beta} \theta J_{0}}{2}}\left\{\left(\lambda-\frac{1}{c}\right) \tilde{D}_{\theta--}+\frac{C}{c} \tilde{A}_{\theta}+\left(\frac{C}{c}-a_{-1}\right) \tilde{D}_{\theta++}+\left(\frac{A+C}{c}\right) \tilde{D}_{\theta--}+\right. \\
& +\frac{C}{c} \tilde{B}_{(\theta-1)-}+\left(\frac{C}{c}-a_{-1}\right) \tilde{C}_{(\theta-1)+}+\left(\frac{C}{c}-a_{-1}\right) \tilde{B}_{(\theta+1)+}+\frac{C}{c} \tilde{C}_{(\theta+1)-}+ \\
& \left.+\left(\frac{C}{c}-a_{-1}\right) \tilde{D}_{(\theta-2)-+}+\left(\frac{C}{c}-a_{-1}\right) \tilde{D}_{(\theta+2)+-}\right\}=0
\end{aligned}
$$

onde a função $t_{2}$ é definida em (B.146) e (B.147) como sendo

$$
\begin{aligned}
t_{2}(\hat{\mu})= & \sum_{\theta \in \mathbb{Z}} e^{\frac{i \mu \hat{\theta} \theta J_{0}}{2}}\left\{\left[\left(\frac{A+B}{c}\right) \tilde{B}_{(\theta-1)+}+\left(\frac{A+C}{c}\right) \tilde{B}_{(\theta-1)-}+\frac{C}{c} \tilde{C}_{(\theta-1)+}+\frac{B}{c} \tilde{C}_{(\theta-1)-}\right]+\right. \\
& +\left[\frac{C}{c} \tilde{B}_{(\theta+1)+}+\frac{B}{c} \tilde{B}_{(\theta+1)-}+\left(\frac{A+B}{c}\right) \tilde{C}_{(\theta+1)+}+\left(\frac{A+C}{c}\right) \tilde{C}_{(\theta+1)-}\right]+ \\
& +\left(\frac{B}{c} \tilde{D}_{(\theta-2)+-} \frac{C}{c} \tilde{D}_{(\theta-2)-+}\right)+\left(\frac{C}{c} \tilde{D}_{(\theta+2)+-} \frac{B}{c} \tilde{D}_{(\theta+2)-+}\right)+ \\
& \left.+\left(\tilde{A}_{\theta}+\tilde{D}_{\theta++}+\frac{A}{c} \tilde{D}_{\theta+-}+\frac{A}{c} \tilde{D}_{\theta-+}+\tilde{D}_{\theta--}\right)\right\}
\end{aligned}
$$

A independência linear dos vetores do conjunto $e^{\frac{i \alpha \theta J_{0}}{2}}, \operatorname{com} \theta \in \mathbb{Z}$, implica cada um dos coeficientes de $e^{\frac{i \Lambda \theta J_{0}}{2}}$ nas nove equações acima ser nulo. A fim de simplificar o cálculo dos autovalores, note que os termos $\tilde{A}_{\theta}, \theta \in \mathbb{Z}$, aparecem somente em (B.156) (notar o cancelamento de $\tilde{A}_{\theta}$ nas equações (B.161) e (B.164)), fornecendo o autovalor

$$
\lambda_{T 1}=\frac{1}{c},
$$

que é infinitamente degenerado como os demais autovalores a serem determinados. Em seguida, o conjunto de equações em (B.162) e (B.163) revela, de imediato, o autovalor

$$
\lambda_{T 2}=\frac{1}{c}-\frac{A}{c} .
$$

Estas duas equações são responsáveis por uma simplificação importante no conjunto das seis equações restantes a serem analisadas. Suas estruturas bloco-diagonais eliminam todos os termos $\tilde{D}_{\theta+-}$ e $\tilde{D}_{\theta-+}$ nas outras equações, deixando para estudar 


$$
\begin{aligned}
& \sum_{\theta \in \mathbb{Z}} e^{\frac{i \hat{\mu} \theta J_{0}}{2}}\left\{\left(\lambda-\frac{1}{c}+\frac{A}{c}\right) \tilde{B}_{\theta+}+a_{1} \tilde{C}_{\theta-}\right\}=0 \\
& \sum_{\theta \in \mathbb{Z}} e^{\frac{i \dot{\mu} \theta J_{0}}{2}}\left\{\left(\lambda-\frac{1}{c}+\frac{A}{c}\right) \tilde{B}_{\theta-}+a_{-1} \tilde{C}_{\theta+}\right\}=0 \\
& \sum_{\theta \in \mathbb{Z}} e^{\frac{i \mu \theta J_{0}}{2}}\left\{\left(\lambda-\frac{1}{c}+\frac{A}{c}\right) \tilde{C}_{\theta+}+a_{1} \tilde{B}_{\theta-}\right\}=0
\end{aligned}
$$

is

$$
\begin{gathered}
\sum_{\theta \in \mathbb{Z}} e^{\frac{i \beta \theta \theta J_{0}}{2}}\left\{\left(\lambda-\frac{1}{c}+\frac{A}{c}\right) \tilde{C}_{\theta-}+a_{-1} \tilde{B}_{\theta+}\right\}=0 \\
\sum_{\theta \in \mathbb{Z}} e^{\frac{i \tilde{\mu} \theta J_{0}}{2}}\left\{\left(\lambda-\frac{1}{c}+\frac{A}{c}\right) \tilde{D}_{\theta++}-a_{1} \tilde{D}_{\theta--}+\left[-\frac{B}{c}\left(1-\frac{B}{c}\right)\right] \tilde{B}_{(\theta-1)-}+\left[\frac{B C}{c^{2}}\right] \tilde{B}_{(\theta-1)+}+\right. \\
+\left[\frac{B}{c}\left(1-\frac{B}{c}\right)-a_{1}\right] \tilde{B}_{(\theta+1)-}+\left[-\frac{B C}{c^{2}}\right] \tilde{B}_{(\theta+1)+}+\left[\frac{B}{c}\left(1-\frac{B}{c}\right)-a_{1}\right] \tilde{C}_{(\theta-1)-}+ \\
\left.+\left[-\frac{B C}{c^{2}}\right] \tilde{C}_{(\theta-1)+}+\left[-\frac{B}{c}\left(1-\frac{B}{c}\right)\right] \tilde{C}_{(\theta+1)-}+\left[\frac{B C}{c^{2}}\right] \tilde{C}_{(\theta+1)+}\right\}=0
\end{gathered}
$$

e

$$
\begin{aligned}
& \sum_{\theta \in \mathbb{Z}} e^{\frac{i a \theta, J_{0}}{2}}\left\{\left(\lambda-\frac{1}{c}+\frac{A}{c}\right) \tilde{D}_{\theta--}-a_{-1} \tilde{D}_{\theta++}+\left[\frac{B C}{c^{2}}\right] \tilde{B}_{(\theta-1)-}+\left[-\frac{C}{c}\left(1-\frac{C}{c}\right)\right] \tilde{B}_{(\theta-1)+}+\right. \\
& +\left[-\frac{B C}{c^{2}}\right] \tilde{B}_{(\theta+1)-}+\left[\frac{C}{c}\left(1-\frac{C}{c}\right)-a_{-1}\right] \tilde{B}_{(\theta+1)+}+\left[-\frac{B C}{c^{2}}\right] \tilde{C}_{(\theta-1)-}+ \\
& \left.+\left[\frac{C}{c}\left(1-\frac{C}{c}\right)-a_{-1}\right] \tilde{C}_{(\theta-1)+}+\left[\frac{B C}{c^{2}}\right] \tilde{C}_{(\theta+1)-}+\left[-\frac{C}{c}\left(1-\frac{C}{c}\right)\right] \tilde{C}_{(\theta+1)+}\right\}=0 .
\end{aligned}
$$

Os pares (B.168) e (B.171), (B.169) e (B.170) são bloco-diagonais, com autovalores

$$
\lambda_{T 3}=\frac{1}{c}-\frac{A}{c}+\sqrt{a_{1} a_{-1}}
$$

e

$$
\lambda_{T}=\frac{1}{c}-\frac{A}{c}-\sqrt{a_{1} a_{-1}} .
$$

Neste processo de diagonalização, esta última etapa elimina todos os termos $\tilde{B}_{\theta-}, \tilde{B}_{\theta+}, \tilde{C}_{\theta-}$ e $\tilde{C}_{\theta+}$, com $\theta \in \mathbb{Z}$, das equações restantes, (B.172) e (B.173). Como conseqüência, estas duas equações adquirem uma forma idêntica aos pares (B.168) e (B.171) (ou (B.169) e (B.170)), o que leva aos mesmos autovalores já calculados. Em suma, 


$$
\begin{gathered}
\lambda_{T 1}=\frac{1}{c}, \\
\lambda_{T 2}=\frac{1}{c}-\frac{A}{c}, \\
\lambda_{T 3}=\frac{1}{c}-\frac{A}{c}+\sqrt{a_{1} a_{-1}}
\end{gathered}
$$

e

$$
\lambda_{T}=\frac{1}{c}-\frac{A}{c}-\sqrt{a_{1} a_{-1}},
$$

sendo que (B.179) é o menor dos autovalores transversais.

\section{B.11- Equação auxiliar - II}

Considere

$$
f(X, Y, Z):=\prod_{\alpha=1}^{n} \cosh \left(X+Y \mu_{\alpha}+Z s_{\alpha}\right)
$$

onde $\left\{\mu_{\alpha}\right\}$ e $\left\{s_{\alpha}\right\}$ são spins de Ising. Sejam

$n_{\uparrow \uparrow} \quad$ o número de configurações $\quad \mu_{\alpha}=+1 \quad$ e $\quad s_{\alpha}=+1 \quad, \quad$ com $\alpha \in\{1, \cdots, n\}$,

$n_{\uparrow \downarrow} \quad$ o número de configurações $\quad \mu_{\alpha}=+1$ e $s_{\alpha}=-1 \quad, \quad$ com $\alpha \in\{1, \cdots, n\}$,

$n_{\downarrow \uparrow} \quad$ o número de configurações $\quad \mu_{\alpha}=-1 \quad$ e $\quad s_{\alpha}=+1 \quad$, com $\alpha \in\{1, \cdots, n\}$,

$n_{\downarrow \downarrow} \quad$ o número de configurações $\quad \mu_{\alpha}=-1 \quad$ e $\quad s_{\alpha}=-1 \quad, \quad$ com $\alpha \in\{1, \cdots, n\}$,

donde segue

$$
\begin{aligned}
& n_{\uparrow \uparrow}=\frac{1}{4}\left(n+\hat{\mu}+\hat{s}+q_{s \mu}\right), \\
& n_{\uparrow \downarrow}=\frac{1}{4}\left(n+\hat{\mu}-\hat{s}-q_{s \mu}\right), \\
& n_{\downarrow \uparrow}=\frac{1}{4}\left(n-\hat{\mu}+\hat{s}-q_{s \mu}\right), \\
& n_{\downarrow \downarrow}=\frac{1}{4}\left(n-\hat{\mu}-\hat{s}+q_{s \mu}\right),
\end{aligned}
$$

$\operatorname{com} q_{s \mu}:=\sum_{\alpha=1}^{n} s_{\alpha} \mu_{\alpha}$. Então, 


$$
\begin{aligned}
f(X, Y, Z)= & \cosh ^{n_{\uparrow \uparrow}}(X+Y+Z) \cosh ^{n_{\uparrow \downarrow}}(X+Y-Z) \cosh ^{n_{\downarrow \uparrow}}(X-Y+Z) \cosh ^{n_{\downarrow \downarrow}}(X-Y-Z) \\
= & {[\cosh (X+Y+Z) \cosh (X+Y-Z)]^{\frac{n+\hat{\dagger}}{4}}[\cosh (X-Y+Z) \cosh (X-Y-Z)]^{\frac{n-\mu}{4}} \times } \\
& \times\left[\frac{\cosh (X+Y+Z)}{\cosh (X-Y+Z)}\right]^{\frac{s+q_{s}}{4}}\left[\frac{\cosh (X+Y-Z)}{\cosh (X-Y-Z)}\right]^{\frac{3+q_{s}}{4}}\left[\frac{\cosh (X+Y+Z)}{\cosh (X-Y+Z)}\right]^{\frac{3-q_{s \mu}}{4}} \times \\
& \times\left[\frac{\cosh (X+Y-Z)}{\cosh (X-Y-Z)}\right]^{\frac{\hat{s}-q_{s \mu}}{4}}
\end{aligned}
$$

Sendo

$$
\tanh ^{-1}(\tanh x \tanh y)=\frac{1}{2} \ln \left[\frac{\cosh (x+y)}{\cosh (x-y)}\right]
$$

tem-se, por fim,

$$
\begin{aligned}
f(X, Y, Z)= & {[\cosh (X+Y+Z) \cosh (X+Y-Z) \cosh (X-Y+Z) \cosh (X-Y-Z)]^{\frac{n}{4}} \times } \\
& \times\left[\frac{\cosh (X+Y+Z) \cosh (X+Y-Z)}{\cosh (X-Y+Z) \cosh (X-Y-Z)}\right]^{\frac{\frac{a}{4}}{4}} e^{\frac{1}{2}\left(\hat{s}+q_{s \mu}\right) \tanh ^{-1}[\tanh (X+Y) \tanh (Z)]} \times \\
& \times e^{\frac{1}{2}\left(\hat{s}-q_{s \mu}\right) \tanh ^{-1}[\tanh (X-Y) \tanh (Z)]}
\end{aligned}
$$

Em particular, usando (B.183),

$$
\begin{aligned}
f(X, Y, 0) & =[\cosh (X+Y) \cosh (X-Y)]^{\frac{n}{2}}\left[\frac{\cosh (X+Y)}{\cosh (X-Y)}\right]^{\frac{\hat{a}}{2}} \\
& =[\cosh (X+Y) \cosh (X-Y)]^{\frac{n}{2}} e^{\hat{\mu} \tanh { }^{-1}[\tanh X \tanh Y]}
\end{aligned}
$$

\section{B.12- Fase paramagnética}

\section{B.12.1- Altos campos aleatórios}

A função de Bessel modificada,

?

$$
I_{\nu}(z):=\sum_{k=0}^{\infty} \frac{\left(\frac{z}{2}\right)^{2 k+\nu}}{\Gamma(k+\nu+1) k !}
$$

tem uma representação integral

$$
I_{\nu}(z):=\frac{\left(\frac{z}{2}\right)^{\nu}}{\sqrt{\pi} \Gamma\left(\nu+\frac{1}{2}\right)} \int_{0}^{\pi} d \theta e^{ \pm z \cos \theta} \sin ^{2 \nu} \theta, \quad \Re \nu>-\frac{1}{2}
$$

Quando o índice $\nu$ é inteiro, uma simplificação da equação acima é possível, resultando em 


$$
I_{m}(z)=\frac{1}{\pi} \int_{0}^{\pi} d \theta e^{z \cos \theta} \cos (m \theta), \quad m \in \mathbb{Z}
$$

Munido desta equação, percebe-se que a solução da equação (3.50),

$$
A=c e^{A-c} I_{m}(c-A),
$$

para grandes valores de $m$ é $A=0$. Com efeito, como $A>0$ (vide Apêndice B.6) pois $A=0$ claramente não satisfaz (B.189) para $c>1$, o argumento da função de Bessel modificada pertence ao intervalo $[0, c) ;$ logo, usando (B.187),

$$
\left|I_{m}(c-A)\right| \leq \frac{\left(\frac{c-A}{2}\right)^{m}}{\sqrt{\pi} \Gamma\left(m+\frac{1}{2}\right)} \int_{0}^{\pi} d \theta\left|e^{c \cos \theta} \sin ^{2 m} \theta\right| \leq \frac{\sqrt{\pi}\left(\frac{c}{2}\right)^{m} e^{c}}{\Gamma\left(m+\frac{1}{2}\right)}
$$

que tende a zero quando $m \rightarrow \infty$. Neste limite, pois, de (B.189), $I_{m}(c-A) \rightarrow 0$ e $A \rightarrow 0$ para $c$ fixo ${ }^{18}$. Então, o autovalor paramagnético

$$
\lambda_{P}=\frac{1}{c}-\frac{A}{c}-\frac{e^{A-c}}{2}\left[I_{m+1}(c-A)+I_{m-1}(c-A)\right]
$$

tende a $1 / c$ quando $m \rightarrow \infty$.

\section{B.12.1- Campo aleatório nulo}

Para $m=0$, as equações a serem considerada são

$$
A=c e^{A-c} I_{0}(c-A)
$$

e

$$
\lambda_{P}=\frac{1}{c}-\frac{A}{c}-e^{A-c} I_{1}(c-A) .
$$

Introduzindo a notação $\bar{t}:=c-A$, e eliminando o parâmetro $c$ em (B.193) através de (B.192), vem

$$
\lambda_{P}=\frac{1}{\bar{t}}\left\{1-\left[e^{-\bar{t}} I_{0}(\bar{t})+\bar{t} e^{-\bar{t}} I_{0}(\bar{t})+\bar{t} e^{-\bar{t}} I_{1}(\bar{t})\right]\right\}
$$

Defina, agora,

$$
p(t):=e^{-t} I_{0}(t)+t e^{-t} I_{0}(t)+t e^{-t} I_{1}(t) .
$$

\footnotetext{
${ }^{18}$ A função de Bessel modificada não é identicamente nula para $m \rightarrow \infty$, mas uma vez fixado o argumento, $o$ índice $m$ pode ser tomado suficientemente grande a ponto do valor da função poder ser suficientemente pequeno nesse argumento.
} 
É importante ressaltar aqui que $t$ é uma variável, enquanto $\bar{t}$ é um número fixo, solução da equação (B.192); $p(\vec{t})$ é o termo entre colchetes em (B.194).

A aplicação $p$ é crescente para $t>0$. Para provar este fato, enuncia-se, inicialmente, o

Lema B.1: Para qualquer $t \in \mathbb{R}$ e $\nu \in \mathbb{Z}$, tem-se

$$
\frac{d I_{\nu}(t)}{d t}=I_{\nu-1}(t)-\frac{\nu}{t} I_{\nu}(t)
$$

Este lema é demonstrado no Apêndice B.13.

Uma derivação de $p$ leva a

$$
\frac{d p(t)}{d t}=e^{-t}\left[2 I_{1}(t)+t \frac{d I_{1}(t)}{d t}-t I_{0}(t)\right]
$$

e recorrendo ao lema B.1, é imediato que

$$
\frac{d p(t)}{d t}=e^{-t} I_{1}(t)
$$

que é positiva para $t>0$. Logo, a aplicação $p$ é monotonicamente crescente nesta região; ademais, ela é contínua, donde segue, para $t>0$, que

$$
p(t)>\lim _{x \rightarrow 0^{+}} p(x)=p(0)=1
$$

Então,

$$
\lambda_{P}<0, \quad \bar{t}>0 .
$$

O ponto $\bar{t}=0$ pode ser tratado separadamente. Impondo este caso, equivalente a $A=c$, em (B.193), chega-se também a um autovalor paramagnético negativo,

$$
\lambda_{P}=\frac{1}{c}-1<0, \quad(A=c),
$$

para $c>1$.

\section{B.13- Prova de lema B.1}

Lema B.1: Para qualquer $t \in \mathbb{R}$ e $\nu \in \mathbb{Z}$, tem-se

$$
\frac{d I_{\nu}(t)}{d t}=I_{\nu-1}(t)-\frac{\nu}{t} I_{\nu}(t)
$$

Prova: Desenvolvendo 


$$
\begin{aligned}
e^{\frac{z}{2}\left(t+t^{-1}\right)} & =e^{\frac{z t}{2}} e^{\frac{z}{2 t}} \\
& =\sum_{m=0}^{\infty} \sum_{n=0}^{\infty} \frac{\left(\frac{z t}{2}\right)^{m}}{m !} \frac{\left(\frac{z}{2 t}\right)^{n}}{n !} \\
& =\sum_{m=0}^{\infty} \frac{\left(\frac{z}{2}\right)^{2 m}}{m ! m !}+\underbrace{\sum_{r=1}^{\infty} t^{r} \sum_{n=0}^{\infty} \frac{\left(\frac{z}{2}\right)^{2 n+r}}{(n+r) ! n !}}_{m>n}+\underbrace{\sum_{r=1}^{\infty} t^{-r} \sum_{m=0}^{\infty} \frac{\left(\frac{z}{2}\right)^{2 m+r}}{(m+r) ! m !}}_{n>m},
\end{aligned}
$$

chega-se a

$$
e^{\frac{z}{2}\left(t+t^{-1}\right)}=\sum_{k \in \mathbb{Z}} t^{k} I_{k}(z)
$$

Derivando ambos membros de (B.204) em relação a $t$,

$$
\begin{aligned}
\sum_{k \in \mathbb{Z}} k t^{k-1} I_{k}(z) & =\frac{z}{2}\left(1-\frac{1}{t^{2}}\right) e^{\frac{z}{2}\left(t+t^{-1}\right)} \\
& =\frac{z}{2}\left(1-\frac{1}{t^{2}}\right) \sum_{k \in \mathbb{Z}} t^{k} I_{k}(z)
\end{aligned}
$$

tem-se

$$
I_{k-1}(z)-I_{k+1}(z)=\frac{2 k}{z} I_{k}(z)
$$

casando os coeficientes de $t^{k}$.

Por outro lado, derivando (B.186) termo a termo, chega-se a

$$
\frac{d I_{\nu}(z)}{d z}=I_{\nu+1}(z)+\frac{\nu}{z} I_{\nu}
$$

que, usando (B.206), obtém-se o resultado desejado. 


\section{Referências Bibliográficas}

\section{Capítulo 1}

[1] Hohenberg PC, Halperin BI, Rev. Mod. Phys. 49, 435 (1977)

[2] Israel RB, Convexity in the theory of lattice gases, Princeton University Press (1979)

[3] Wilson KG, Phys. Rev. B 4, 3174 (1971)

[4] Wilson KG, Phys. Rev. B 4, 3184 (1971)

[5] Harris AB, J. Phys. C 7, 1671 (1974)

[6] Imry Y, Ma S-K, Phys. Rev. Lett. 35, 1399 (1975)

[7] Edwards SF, Anderson PW, J. Phys. F 5, 965 (1975)

[8] Sherrington D, Kirkpatrick S, Phys. Rev. Lett. 35, 1792 (1975)

[9] Selke W, in Phase Transitions and Critical Phenomena vol 15, ed C Domb and JL Lebowitz (London: Academic), 1 (1992)

[10] Yoshimori A, J. Phys. Soc. Japan 14, 807 (1959)

[11] Villain J, J. Phys. Chem. Solids 11, 303 (1959)

[12] Kaplan TA, Phys. Rev. 116, 888 (1959)

[13] Godrèche C, Luck JM, J. Phys. A 33, 9141 (2000)

\section{Capítulo 2}

[14] Hohenberg PC, Halperin BI, Rev. Mod. Phys. 49, 435 (1977)

[15] Bouchaud J-P, J. Physique I France 2, 1705 (1992)

[16] Cugliandolo LF, Dean DS, J. Phys. A 28, 4213 (1995)

[17] Cugliandolo LF, Kurchan J, Parisi G, J. Physique I France 4, 1641 (1994)

[1.8] Cugliandolo LF, Kurchan J, Phys. Rev. Lett. 71, 173 (1994)

[19] Cugliandolo LF, Kurchan J, J. Phys. A 27, 5749 (1994)

[20] Cugliandolo LF, Kurchan' J, Philos. Mag. B 71, 501 (1995)

[21] Franz S, Mézard M, Europhys. Lett. 26, 209 (1994) 
[22] Franz S, Mézard M, Physica A 209, 1 (1994)

[23] Ritort F, Phys. Rev. Lett. 75, 1190 (1995)

[24] Zippold W, Kühn R, Horner H, Eur. Phys. J. B 13, 531 (2000)

[25] Godrèche C, Luck JM, J. Phys. A 33, 9141 (2000)

[26] Picone A, Henkel M, J. Phys. A 35, 9141 (2002)

[27] Cannas SA, Stariolo DA, Tamarit FA, Physica A 294, 362 (2001)

[28] Crisanti A, Ritort F, J. Phys. A 36, R181 (2003)

[29] Cugliandolo LF, Dean DS, J. Phys. A 28, L453 (1995)

[30] Stariolo DA, Cannas SA, Phys. Rev. B 60, 3013 (1999)

[31] Onsager L, Phys. Rev. 65, 117 (1944)

[32] Kac M, Phys. Today 17, 40 (1964)

[33] Berlin TH, Kac M, Phys. Rev. 86, 821 (1952)

[34] Lewis HW, Wannier GH Phys. Rev. 88, 682 (1952)

[35] Lewis HW, Wannier GH Phys. Rev. 90, 1131E (1953)

[36] Stanley HE, Phys. Rev. 176, 718 (1968)

[37] Helfand E, Phys. Rev. 183, 562 (1969)

[38] Kac M, Thompson CJ Phys. Norv. 5, 163 (1971)

[39] Shcherbina M, Theor. Math. Phys. 77, 1323 (1988)

[40] Kunz H, Zumbach G, J. Phys. A 25, 6155 (1992)

[41] Khorunzhy A, Khoruzhenko B, Pastur L, Shcherbina M, in Phase Transitions and Critical Phenomena vol 15, ed. C Domb e JL Lebowitz (London: Academic), 73 (1992)

[42] Joyce GS, Phys. Rev. 146, 349 (1966)

[43] Joyce GS, Phase Transitions and Critical Phenomena, Vol. 2, ed. C. Domb, M. S. Green (London: Academic Press), 375 (1972)

[44] Kosterlitz JM, Thouless DJ, Jones RC, Phys. Rev. Lett. 36, 1217 (1976)

[45] Kalok L, Obermair GM, J. Phys. C 9, 819 (1976)

[46] Hornreich RM, Luban M, Shtrikman S, Phys. Rev. Lett. 35, 1678 (1975)

[47], Hornreich RM, Luban M, Shtrikman S, Physica A 86, 465 (1977)

[48] Selke W, in Phase Transitions and Critical Phenomena vol 15, ed C Domb and J L Lebowitz (London: Academic), 1 (1992) 


\section{Capítulo 3}

[49] Edwards SF, Anderson PW, J. Phys. F 5, 965 (1975)

[50] Sherrington D, Kirkpatrick S, Phys. Rev. Lett. 35, 1792 (1975)

[51] Imry Y, Ma S-K, Phys. Rev. Lett. 35, 1399 (1975)

[52] Fishman S, Aharony A, J. Phys. C 12, L729 (1979)

[53] Schneider T, Pytte E, Phys. Rev. B 15, 3 (1977)

[54] Aharony A, Phys. Rev. B 18, 3318 (1978)

[55] de Almeida JRL, Bruinsma R, Phys. Rev, B 35, 7267 (1987)

[56] Pastor AA, Dobrosavljević V, Horbach ML, Phys. Rev. B 66, 014413 (2002)

[57] Hase MO, de Almeida JRL, Salinas SR, Eur. Phys. J. B, aceito para publicação (2005)

[58] Cardy JL, Phys. Rev. B 29, 505 (1984)

[59] Belanger DP, in Spin Glasses and Random Fields, ed AP Young, 251 (1997)

[60] Berretti A, J. Stat. Phys. 38, 483 (1985)

[61] Imbrie JZ, Phys. Rev. Lett. 53, 1747 (1984)

[62] Nattermann T, in Spin Glasses and Random Fields, ed AP Young, 277 (1997)

[63] Belanger DP, Young AP, J. Magn. Magn. Mater. 100, 272 (1991)

[64] Salinas SR, Wreszinski WF, J. Stat. Phys. 41, 299 (1985)

[65] van Hemmen JL, Phys. Rev. Lett. 49, 409 (1982)

[66] van Hemmen, Lecture Notes in Physics, vol. 192, 203 (1983)

[67] Bruinsma R, Phys. Rev. B 30, 289 (1984)

[68] Parisi G, Sourlas N, Phys. Rev. Lett. 43, 744 (1979)

[69] van Hemmen JL, Palmer RG, J. Phys. A 15, 3881 (1982)

[70] van Hemmen JL, Palmer RG, J. Phys. A 12, 563 (1979)

[71] de Almeida JRL, Thouless DJ, J. Phys. A 11, 983 (1978)

[72] Parisi G, J. Phys. A 13, L115 (1980)

[73] Mézard M, Parisi G, Sourlas N, Toulouse G, Virasoro M, Phys. Rev. Lett. 52, 1156 (1984)

[74] Mézard M, Parisi G, Virasoro M, Spin-Glass Theory and Beyond, World Scientific, 1987.

[75] Binder K, Young AP, Rev. Mod. Phys. 58, 801 (1986)

[76] Talagrand M, C. R. Acad. Sc. Paris 337, 111 (2003)

[77] Viana L, Bray AJ, J. Phys. C 18, 3037 (1985)

[78] Kanter I, Sompolinsky H, Phys. Rev. Lett. 58, 164 (1987)

[79] Mézard M, Parisi G, Europhys. Lett. 3, 1067 (1987)

[80] Mottishaw P, de Dominicis C, J. Phys. A 20, L375 (1987)

[81] de Almeida JRL, de Dominicis C, Mottishaw P, J. Phys. A 21, L693 (1988) 